\title{
COMPUTATIONAL TECHNIQUES IN \\ GAMMA-RAY SKYSHINE ANALYSIS
}

$$
\text { by }
$$

Darin Lynn George

B.S., Kansas State University, 1986

\section{A MASTER'S THESIS}

submitted in partial fulfillment of the requirements for the degree

\section{MASTER OF SCIENCE}

\section{Department of Nuclear Engineering KANSAS STATE UNIVERSITY \\ Manhattan, Kansas \\ 1988}

Approved by:

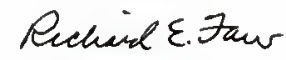

Major Professor 
TABLE OF CONTENTS

Page

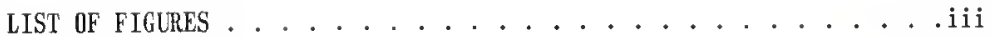

LIST 0F TABLES. . . . . . . . . . . . . . . . . . . . . . . .

1.0 INTRDDUCTION . . . . . . . . . . . . . . 1

2.0 REVIEW OF COYPUTATIONAL SKYSHINE STUDIES ......... 5

2.1 Monte Carlo Studies . . . . . . . . . . . . . 6

2.2 Single-Scatter Studies. . . . . . . . . . . . . . 9

2.3 0ther Numerical Skyshine Studies. . . . . . . . 12

2.4 Experimental hork............... 15

3.0 COYPUTATION OF SKYSHINE EXPOSURE RATES FOR SIMPLE GEOYETRIES . . 18

3.1 The Single-Scatter and Buildup Model. . . . . . . . . 19

3.1.1 Interactions Considered. . . . . . . . . . . 21

3.1.2 Application of Buildup . . . . . . . . . . 28

3.2 The Exposure Rate Integral Equation . . . . . . . 32

3.3 Application of the Exposure Rate Integral Equation to a

Silo Geometry . . . . . . . . . . 38

3.3.1 Coordinate System. . . . . . . . . . . . . . 40

3.3 .2 Limits of Integration. . . . . . . . . . . 42

3.3.3 Numerical Evaluation .....................

3.4 Application of the Exposure Rate Integral Equation to a

Wall Geometry ............... 55

3.4.1 Coordinate Systems . . . . . . . . . . . 62

3.4 .2 Limits of Integration. . . . . . . . . . 75

3.4.3 Numerical Evaluation . . . . . . . . . . .112

4.0 RESULTS AND VALIDATION OF THE CODES DEVELOPED IN THIS WORK . . . .123

4.1 Comparison of SILOGP to Other Methods and Benchmark Data. .125

4.1.1 Comparisons to Benchmark Study . . . . . . . . 125

4.1.2 Comparisons to ANSI Standard . . . . . . . . . . 131

4.1.3 Comparisons to Buildup Factors in the $2 \pi$ Problem . .136

4.1.4 Comparisons to MicroSkyshine . . . . . . . . . . 138

4.2 Comparison of WALLGP to Other llethods . . . . . . . 148

4.2.1 Comparisons to ANSI Standard . . . . . . . . . . .149

4.2.2 Comparisons to Buildup Factors in the $2 \pi$ Problem . .150

4.2.3 Comparisons to IlicroSkyshine . . . . . . . . . .154

5.0 CONCLUSIONS AND SUgGESTIONS FOR FURTHER STUDY. . . . . . . . . .

REFERENCES. . . . . . . . . . . . . . . . . . . . . . . . . . . 
ACKNOWLEDGEMENTS. . . . . . . . . . . . . . . . . . . . . . . . . .

APPENDIX A: The Computer Program SILOGP Developed in this Work for the Problem of a Point Gamma- Ray Source in a Cylindrical Silo . . . . . . . . . . . . . . 177

APPENDIX B: The Computer Program WALLGP Developed in this Work for the Problem of a Point Gamma-Ray Source Behind a Semi-Infinite wall . . . . . . . . . . . 185

APPENDIX C: Subroutines Required by SILOGP and WALLG. . . . . . . 203 APPENDIX D: Sample Problem Input and 0utput from SILOGP. . . . . .221 APPENDIX E: Sample Problem Input and 0utput from WALLGP. . . . . .225 


\section{LIST OF FIGURES}

Figure

Page

3-1 Representation of the single-scatter and buildup method . . 22

3-2 Mass interaction coefficients for gamma rays in air. Data from (Ch84)............... 24

3-3 Application of buildup to the problem of a slab shield over a point source............... . . 30

3-4 Distances involved in the single-scatter geometry: (a) no overhead shield; (b) overhead concrete roof present. . . 33

3-5 Simplified calculational geometry for the case of a point source within a silo: (a) no overhead shield;

(b) overhead concrete roof present. [From (Ro80)]. . . . 39

3-6 Coordinate system for the single-scatter model as applied to the silo geometry. [From (Ro82)] . . . . . . 41

3-7 Comparison of exposures computed by the computer code ShY8 using the Berger and geometric progression (GP) buildup formulas.

3-8 Simplified calculational geometry for the case of a point source behind a semi-infinite wall: (a) no overhead shield; (b) overhead concrete roof present . . . 58

3-9 Coordinate system for the source and detector before application of the single-scatter geometry to the wall

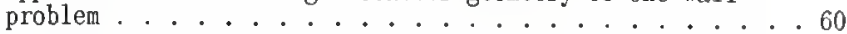

3-10 Coordinate system for the source and detector, presented in orthographic views based on the XYZ axes . . . 61

3-11 Translation of wall geometry from Cartesian to cylindrical coordinates: first step, rotation in $\mathrm{XY}$ plane through an angle $\theta . . . . . . . . . . . .63$

3-12 Translation of wall geometry from Cartesian to cylindrical coordinates: new coordinates of source and detector in $X^{\prime} Y^{\prime} Z^{\prime}$ system created by first rotation . . . 64

3-13 Translation of wall geometry from Cartesian to cylindrical coordinates: second step, rotation in $X^{\prime} Z^{\prime}$ plane through an angle $\xi . . . . . . . . . . .666$ 
3-14 Translation of wall geometry from Cartesian to cylindrical coordinates: new coordinates of source and detector in $X^{\prime \prime} Y " Z^{\prime \prime}$ system created by second rotation. . . . 67

3-15 Translation of wall geometry from Cartesian to cylindrical coordinates: relationship between the $X^{\prime \prime} Y " Z$ " system and the cylindrical system. . . . . . . . . 69

3-16 Cartesian components of the path length $a_{c}$ through the concrete shield and the path length $\bar{a}$ between the source and the scattering volume $\mathrm{dV}$. .........71

3-17 Definition of the scattering zone used to formulate limits of integration for the wall geometry ......76

3-18 Orientation of the scattering zone in the cylindrical coordinate system ................. 77

3-19 Types of wall-source-detector geometries, viewed in the Cartesian and cylindrical frames of reference . . . . . . 80

3-20 Cylindrical surfaces used to determine limits of integration on $\mathrm{z} \ldots \ldots \ldots 1$

3-21 Geometry used to find limits of integration over $z$ using a radial $r$ normal to the boundary of the scattering zone over the source ...........83

3-22 Geometry used to find limits of integration over z using a radial $\mathrm{r}$ normal to the boundary of the scattering zone over the detector ..........85

3-23 The set of radials normal to a half plane bordering the scattering zone............. 86

3-24 Geometry defining the radial distance $r$ from axial position $z$ to the top of the wall ....... 87

3-25 Alternate limits of integration over $\mathrm{z}$, based on the mean-free-path cutoff . . . . . . . . . . 91

3-26 Cylindrical surfaces defining limits of integration over $\phi$ for known $r$ and $z$. . . . . . . . . 96

3-27 Geometry defining limits of integration over $\phi$ for a case in which the wall extends above neither source nor detector. 
3-28 Limits of integration over $\phi$ for source-detector geometries in which the wall extends above neither source nor detector . . . . . . . . . . . . . . 101

3-29 Limits of integration over $\phi$ in a source- detector geometry for which the wall extends over the source and the $\phi$ integral breaks into two integrals over separate regions ......................... . . . . . .

3-30 Limits of integration over $\phi$ in a source-detector geometry for which the wall extends over the source, but the $\phi$ integral does not split into two separate

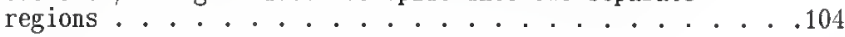

3-31 Geometry defining the $z$ coordinate at which the $\phi$ integration may break into two integrals. . . . . . 106

3-32 Contour plot of the limits of integration in the wall geometry for increasing values of the radial coordinate $\mathrm{r}$. The source and detector lie in a plane normal to the wall; $x_{s}=x_{d}=y_{\delta}=y_{d}=10 \mathrm{~m} . \ldots . . .113$

3-33 Contour plot of the limits of integration in the wall geometry for increasing values of the radial coordinate $r$. The source and detector are offset by $z_{d}=5 \mathrm{~m}$; the wall extends over neither source nor detector. $x_{\delta}=x_{d}$ $=\mathrm{y}_{\mathrm{s}}=\mathrm{y}_{\mathrm{d}}=10 \mathrm{~m} \ldots \ldots \ldots \ldots \ldots \ldots \ldots$

3-34 Contour plot of the limits of integration in the wall geometry for increasing values of the radial coordinate $r$. The source and detector lie in a plane normal to the wall, and the wall extends over the source in the cylindrical frame of reference. $x_{s}=x_{d}=y_{\delta}=10 \mathrm{~m}$; $\mathrm{yd}_{\mathrm{d}}=40 \mathrm{~m} ; \mathrm{z}_{\mathrm{d}}=0 \ldots \ldots \ldots \ldots \ldots \ldots . . \ldots \ldots$

4-1 Comparison of measured data from the benchmark skyshine experiment, discrete ordinates calculations by $\mathrm{DOT}$, and calculated results of the point-kernel code SILOGP. . . . .127

4-2 Problem geometry for ANSI Reference Problem I.1. [From (AN87)] . . . . . . . . . . . . . . . . .

4-3 Comparison of SILOGP results for ANSI Reference Problem I.1 to reference values and results of the line-beam response function program MicroSkyshine.

4-4 Comparison of SILOGP results for the $2 \pi$ problem of Faw and Shultis (Fa87) to the moments-method reference values. 
4-5 Comparison of MicroSkyshine results for the $2 \pi$ problem of Faw and Shultis (Fa87) to the moments-method

reference values. . . . . . . . . . . 140

4-6 Comparison of results for the benchmark problem computed by MicroSkyshine, $G^{3}$ and SILOGP. . . . . . . 143

4-7 Comparison of WALLGP results for ANSI Reference Problem I.1 to reference values and results of the line-beam response function program MicroSkyshine . . . . . . . .151

4-8 Comparison of WALLGP results for the $2 \pi$ problem of Faw and Shult is (Fa87) to the moments-method reference values. . . . . . . . . . . . . . 153

4-9 Comparison of dose estimates computed by MicroSkyshine,

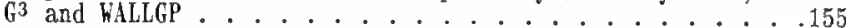

4-10 Comparison of the regions of space in the wall geometry in which WALLGP and MicroSkyshine allow photons to undergo their first scatter .............. . . . . . . . .

5-1 Normalized graphs of the functions integrated by SILOGP . .169

5-2 Normalized graphs of the functions integrated by KALLGP . .170

D-1 Illustration of the problem geometry modeled by the sample input file for SILOGP. . . . . . . . . . .224

E-1 Illustration of the problem geometry modeled by the sample input file for WALLGP. . . . . . . . 228 


\section{LIST OF TABLES}

Table

Page

3-1 Comparison of methods of numerical integration as applied to the computer code SKY. The exposures are for a $60 \mathrm{Co}$ point source on the axis of an open silo with a solid angle of collimation of $4.683 \mathrm{sr}$. Air density is taken as $1.2 \mathrm{mg} / \mathrm{cm}^{3}$. . . . . . . . . . . 49

3-2 Comparison of methods of numerical integration as applied to the computer code SKY. The exposures are for a ${ }^{60} \mathrm{Co}$ point source on the axis of a silo, shielded by a concrete slab of density $2.13 \mathrm{~g} / \mathrm{cm}^{3}$ and thickness

$21 \mathrm{~cm}$. Air density is taken as $1.2 \mathrm{mg} / \mathrm{cm}^{3}$. . . . . . . 50

3-3 Comparison of mass interaction data used in the computer code SILOGP. The exposures are for a ${ }^{60} \mathrm{Co}$ point source on the axis of an open silo with a solid angle of collimation of $4.683 \mathrm{sr}$. Air density is taken as $1.2 \mathrm{mg} / \mathrm{cm}^{3}$................ . . . 56

3-4 Comparison of mass interaction data used in the computer code SILOGP. The exposures are for a ${ }^{60} \mathrm{Co}$ point source on the axis of a silo, shielded by a concrete slab of density $2.13 \mathrm{~g} / \mathrm{cm}^{3}$ and thickness 21 $\mathrm{cm}$. Air density is taken as $1.2 \mathrm{mg} / \mathrm{cm}^{3}$. . . . . . . . 57

3-5 Limits of integration over the azimuthal angle $\phi$ for known values of $\mathrm{r}$ and $\mathrm{z}$, in source-detector geometries such that $x_{\mathrm{S}}^{\prime \prime}$ or $\mathrm{x}_{\mathrm{d}}^{\prime \prime}$ is negative. . . . . . . . . . 109

3-6 Limits of integration over the azimuthal angle $\phi$ for

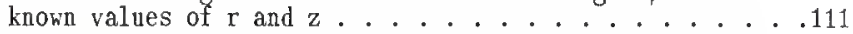

3-7 Test cases used to investigate the convergence of the computer code KALLGP. . . . . . . . . . . . . . . 118

3-8 Behavior of detector responses computed by WLLGP with changes in the mean free path cutoff value. . . . . . . .119

3-9 Behavior of detector responses computed by WALGP with changes in convergence criteria . . . . . . . . . . 122

4-1 Comparison of detector responses computed by the codes MicroSkyshine and SILOGP with changes in collimation

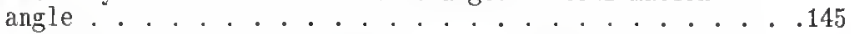

4-2 Comparison of detector responses computed by the codes HicroSkyshine and WALLG with changes in source energy. . .159 
4-3 Comparison of detector responses computed by the codes MicroSkyshine and WALLGP with changes in wall height. . . .161

4-4 Parametric study of detector responses computed by the codes MicroSkyshine and WALLGP. . . . . . . . . 163 


\subsection{INTRODUCTION}

An ongoing concern of the public and of organizations involved with nuclear power or radiation sources is radiation protection. One specific concern, recognized before the advent of nuclear power, is skyshine. In the context of this study, skyshine is defined as directly or indirectly ionizing radiation which scatters in the atmosphere after leaving its source. While a small, efficient shield can block direct radiation from a source to a detector or point of interest, skyshine can avoid the shield entirely and still produce a significant dose at the detector. Even when a direct dose is present, the fraction of the total dose due to skyshine can be substantial.

Recent events emphasize a need for accurate methods to estimate gamma-ray skyshine. After accidents at Three Mile Island and Chernobyl, reviews were made of the techniques used to forecast and estimate the dose to the public and surrounding land from radiation releases of many types. Soon, low-level radioactive waste repositories will be built in several states. Effects on the environment will be a topic of study for every site, and gamma rays are likely to be a large component of any radiation to reach the surroundings. Gamma-ray skyshine will also be present at sites for the storage of spent fuel. In these repositories, air or water convection may be used to carry off waste heat. Air would not shield the fuel as well as water in storage pools, yet photons from fission products could escape from either type of repository to the atmosphere. All of these situations call for methods of estimating exposure rates from gamma rays which reach the point of interest as skyshine.

Another justification for work on this topic is found in the American National Standard for Calculation and Measurement of Direct and Scattered Gamma Radiation from LWR Nuclear Power Plants, ANSI/ANS-6.6.1-1987. This 
document explains that the activation of ${ }^{16} \mathrm{~N}$ in the coolant of boiling water reactors is a cause for concern. As stated in the standard, "... at a BWR the ${ }^{16} \mathrm{~N}$ contribution to the total measured dose rate is the only significant one that changes nearly instantaneously with power level variations." This activity in the coolant depends on water chemistry; at facilities where hydrogen is added directly to the coolant, its presence may cause the activity to increase severalfold. The gamma radiation from ${ }^{16} \mathrm{~N}$ has been detected inside and outside the containment of several BWRs, and is documented in the standard. (AN87)

ANSI/ANS-6.6.1-1987 also provides reference calculations for simple skyshine problems, and recommends that comparisons be made between those calculations and results of computational methods of interest applied to the same problems. The standard states that dose rates should be found for areas outside a plant (or other gamma-ray source) which personnel are expected to occupy regularly, such as construction areas or recreation areas. Heavily populated areas should also be chosen for study. Also, the standard explains requirements for calculations and measurement techniques used to obtain gamma ray dose rates, and suggests ways to treat scattered radiation components of the results. In summary, the standard provides a reference for those who wish to insure that the methods they use are in reasonable agreement with other methods, both in technique and in results. (ANS7)

These concerns and standards promote the search for accurate estimates of gamma-ray skyshine dose. Practical benchmark experiments are often accepted as the best sources of data, but in the case of radiation skyshine, experiments can be time-consuming and impractical. The alternative sources of data widely accepted as standard are computer codes. Programs have been developed specifically for skysline problems, and other general transport codes may be applied to skyshine 
dose estimation. Ideally, an experiment would he performed first to collect data in a real situation. Next, a code would he executed and the results would be checked against the experimental data. When the code results agree well with the benchmark data, the code could then be applied to similar prohlems with confidence in its accuracy, and at less cost than an experiment.

Problems and physical geometries simulated by many codes are simple: open air environments, sources enclosed by a silo or a sphere, free-standing walls, or buildings defined by four walls and a ceiling. These simple systems allow computational effort to concentrate on particle transport and dose computation, instead of geometric prohlems such as changes in material along a particle's direction of travel. Also, the simple geometries can be modeled more easily in practical experiments, so benchmark data should he easier to obtain for comparison.

The numerical methods used in skyshine computer codes include discrete ordinates transport, line-beam techniques and Monte Carlo methods. While these codes can be accurate, most are large and computationally intense, making them difficult to use on a regular hasis. One notable exception is the MicroSkyshine method developed hy Faw and Shultis, which may be implemented on a personal computer and has been shown to be accurate for several practical cases (Fa87). Another method which requires less computational time and effort applies point-kernel techniques and buildup factors to estimate gamma exposure. This approach has been taken by Trubey (Tr61), Kitazume (Ki68) and Roseherry (Roso).

The point-kernel technique, or single scatter and buildup method, is modeled on the basis that each plzoton scatters once, and only once, as it travels from its source to a detector. However, a buildup factor is applied along the path of the 
photon from the point of scatter to the detector. The volume of air wherein the photon scatters may be thought of as a "first-collision source" of scattered photons. Integration over all "source volumes" can provide an estimate of scattered gamma-ray intensity at the point of interest.

Roseberry applied the point-kernel metbod to the case of a point source concealed from a detector by a cylindrical shield, open above the source. A benchmark experiment in this geometry was performed, and comparison of experimental data to Roseberry's numerical results proved that tbe method is useful and accurate, though conservative (Ros0). A second simple skyshine problem involves a point source concealed from a detector by a semi-infinite, perfectly absorbing wall. The point-kernel method is applied to the wall problem in this work as an extension of Roseberry's solution.

This thesis discusses computational techniques used in past and current codes for gamma-ray skysbine analysis. It begins with a review of gamma-ray skyshine studies, most of which have been numerical; one practical benchmark experiment is also described. Next, the single scatter and buildup model is discussed in depth. Photon interactions and the use of buildup factors in this scheme are explained, and an exposure rate equation is derived based on the model. Calculations performed by Roseberry with the model are repeated, using new data and a different integration method. The semi-infinite wall problem is also approached with this model, employing the same data and integration scheme. Finally, the results obtained in both the silo and wall computations are compared to results of other methods, and to benchmark data and ANSI standards where possible. This allows conclusions about the usefulness of the method and the new data presented in this thesis. 


\subsection{REVIEW OF COMPUTATIONAL, SKYSHINE STUDIES}

Before a technique of computing skyshine is presented in this thesis, a review of past work in the field will be useful. Methods which experimenters have found to work well in the past can be used as a starting point in research; for this work, a literature review will act as a starting point.

Research on skyshine is reported in publications dating back to the 1950's. One 1956 report by Zerby (Ze56) explained calculations needed to adjust neutron flux densities and dose rates to accommodate variations in air density. The calculations were applied to measurements made at the Tower Shielding Facility, where a radiation source was suspended in midair and measurements were taken at a second point in the air. Since the measured dose rates and flux densities contained a component due to air-scattered radiation, this work could be considered one of the first on skyshine computation. Note that it is concerned with neutron skyshine, however.

Zerby's report also demonstrates two common methods of computing skyshine quantities. Preliminary computations were performed for the experiment using the Monte Carlo method. The results demonstrated that in nearly all cases, neutrons which had scattered in air three times or less were the major contributors to measured dose. (Ze56) The Monte Carlo method became practical with the introduction of the modern mainframe computer, and is employed in many particle-transport codes. The conclusion that particles which scatter fewer times are more important supports the use of the point-kernel method, in which the point of first scatter is treated as a source for transport to some other point. 
All but one of the numerical skyshine studies discussed in this chapter utilize one of the two techniques named above. Monte Carlo studies will be reviewed first, followed by a discussion of work based upon single-scatter models and a third method involving the single-scatter technique. A fourth section will describe a benchmark experiment and the work associated with it.

\subsection{Monte Carlo Studies}

An early study of skyshine by Monte Carlo computation is that of Lynch et al. (Ly58). The program written in the study was for a simple problem: a monoenergetic line beam of gamma rays (that is, gammas released in one direction at one energy) from a point source placed in an infinite air medium, with no other shielding. Flux densities were reported for different source-detector distances, source energies and beam directions. Tissue dose rates were also computed and reported.

For comparison purposes, Lynch et al. modified the code to use an isotropic source and isotropic scattering without energy degradation. Results were compared to the analytic solution of the Boltzmann equation for the same case, and the average numerical results were within $15 \%$ of the Boltzmann solution; often the error was much less. Computations of single-scatter flux densities were also compared to analytical solutions, and similar accuracy was found in these quantities. For this ideal case, the Monte Carlo method was shown to be useful if statistical methods were used to compute confidence limits on results.

A much more detailed Monte Carlo code package was prepared by Radiation Research Associates in 1969 ( $\mathrm{Ma69}$; Co69). Instead of gamma-ray skyshine, this study concentrated on the $X$-rays and fluorescent light resulting from nuclear 
explosions. Comparing this work to that of the ideal, simple geometry of Lynch et al. demonstrates the advances made in the Monte Carlo method over the decade between the two reports. The RRA codes computed energy deposition as well as flux density; the point source strength could be specified as a function of time, energy, and direction; attenuation coefficients could be made altitude dependent and energy dependent; and flux density could now be computed over time and over changes in air density or altitude.

The first code in the package, named ZAP, used random walks to trace the entire path of each X-ray, scatter by scatter, until its "death." Compton, photoelectric, and pair production interactions, and some coherent scattering, were all accounted for, so that both scattering and absorption of the X-ray could be recorded (Ma69). The second code in the package, PFLASH, received data from ZAP on the energy deposited by X-rays absorbed in air. Using extensive data on the physics of fluorescent light production, this conversion code then created volumetric source terms for fluorescent light to be used by the third code, FLASH. FLASH computed the fluorescent light intensity at a point detector due to the volumetric source created by PFLASH. It performed fluorescent light transport by a backward Monte Carlo method, in which particle histories begin at the detector and "walk backward" to the source. This method allows angular-dependent quantities to be computed in less time than by forward Monte Carlo methods, and also allows time-dependent calculations just as forward calculations do (Co69). In summary, this study by RRA demonstrates the advances made in the method after the work of Lynch et al., and the interactions which would be regularly used for probability calculations in the future. 
Neither of the Monte Carlo studies above involved shields or structures of any sort. Perhaps the first Monte Carlo code to determine the effects of structures on gamma-ray dose was the SKYSHINE program, later modified to become the more versatile SKYSHINE-II code (La79). In both programs, a source was enclosed in a simple building of four walls, a ceiling and a floor. Sections of the walls and ceiling could be "removed" to collimate the radiation. Since the calculations were based upon Monte Carlo line-beam data, the code would obtain dose by integrating the line-beam data over all beams passing through the opening or openings.

The source could be specified as a neutron source, a primary gamma-ray source or a secondary gamma-ray source, each with a spectrum of emitted energies, and anisotropic scattering could be treated in the calculations. Using the results of Zerby and other Monte Carlo studies, the code performed its own computations to find the air-scattered dose rate at each wall, the floor, the ceiling, and other points outside the building. This allowed users to study the effects of building design on radiation dose, whether direct radiation dose or skyshine. In this respect, SKYSHINE was a milestone in the topic of radiation studies.

The MORSE code has been modified many times, with each version given its own capabilities to use in certain problems or to receive data from other programs. The 1984 release, MORSE-SGC/S, is a neutron and gamma Monte Carlo code which solves both shielding and criticality problems. The physical form of the system studied is described with combinatorial geometry, a versatile algorithm which creates region boundaries from planes and conic surfaces. Particle transport is based upon the Boltzmann equation, and performed by "supergroups," energy groups made up of smaller energy groups. To reduce the computer memory required, MORSE-SGC/S separates the entire spectrum of particle energies into 
clusters of energy regions. The code performs calculations on particles in the first cluster, or supergroup, stores the results, then calls in data on the particles in the next lower supergroup to work with. Both the combinatorial geometry and supergroups make the code versatile in terms of possible applications and computers which may be used. (We\$4)

MORSE-SGC/S reports responses to both uncollided radiation and the sum of direct and scattered radiation. This would suggest that it is ideal for gamma-ray skyshine analysis. However, work at Kansas State shows the code requires many gamma-ray histories to estimate the usually low gamma-ray skyshine dose. Also, MORSE cannot satisfactorily simulate point detectors, since Monte Carlo codes must use finite volumes, and small volumes receive few particles to count toward dose. (Sh88) Even with particle weight biasing to reduce the standard deviation of results, uncertainties on such small quantities could make the results useless. This, combined with the long run-times required, may make MORSE-SGC/S impractical for gamma-ray skyshine analysis. Indeed, this argument has been made against the Monte Carlo method in general as applied to gamma-ray skyshine: The number of particle histories required to obtain good statistics can be prohibitive. The method is simple to apply and may be of use for neutron skyshine analysis, but that is not of concern here.

\subsection{Single-Scatter Studies}

As cited by Trubey (Tr61), C. H. Bernard first hypothesized in 1953 that the single-scattered flux density made a good approximation to the total scattered flux density for gamma rays or neutrons. Because buildup and exponential attenuation 
tend to counteract each other, Bernard did not use either. Trubey's work marked an early application of Bernard's model to gamma rays.

Trubey considered a simple problem, a monodirectional, monoenergetic point source of gamma rays in infinite air. This is identical to the line-beam problem used by Lynch et al. (Ly58) for their Monte Carlo research, and the problem was chosen to allow comparisons with their results. Dose rates computed in this manner agreed very well with the Monte Carlo calculations; however, flux densities computed with the single-scatter model were low if the gamma rays backscattered, that is, if the line beam was directed away from the detector and photons could only reach the detector by reversing direction. The use of Trubey's results was limited by the model; any shielding would make the data invalid. Still, as noted by Faw and Shultis (Fa87), Trubey confirmed that most skyshine dose in these cases is due to once-scattered gamma rays.

Kitazume (Ki68) introduced a new single-scatter formula for approximating gamma-ray dose when a point source and detector are in air above ground. This formula allowed the point source to be isotropic or monodirectional. Attenuation of both unscattered and scattered photons was accounted for, and Taylor buildup factors were applied to once-scattered gamma rays to simulate multiple scatters. The formula is very general and has proven useful in later work; Roseberry's formulation (Ros0) was shown to be equivalent to Kitazume's.

Kitazume performed line-beam source calculations to be compared with thase of both Lynch et al. and Trubey. With buildup factors for air unavailable, Kitazume chose to use factors for water instead. The results compared well with the Monte Carlo calculations except for low source energies and large source-detector distances, or low energies and photon beams at large angles from an 
axis between source and detector. Even this disagreed with Lynch et al. by only $20 \%$. The discrepancies became smaller as source energy increased, while Trubey's computations without attenuation and buildup yielded poorer underestimates of the Monte Carlo values as the energy increased. (Ki68) This makes a strong argument for the inclusion of both attenuation and buildup in single-scatter calculations; indeed, their use has become standard.

A notable series of point-kernel programs was developed during the 1960's at Los Alamos for neutron and photon shielding studies. This series, named QAD, consists of several codes which compute uncollided flux densities, dose rates and energy depositions from a volumetric source. While the codes compute only line-of-sight quantities, the geometries they can use are complex. QAD employs a combinatorial geometry in which Cartesian, cylindrical and spherical surfaces are combined to describe the physical system being modeled. The regions may be of different materials or mixtures of materials. Also, QAD codes can recreate buildup factors from curves fit to experimental data, and apply them to paths through each region, regardless of material. (Ma67)

The QAD codes themselves have no use in single-scatter studies. Their importance lies in the offshoot family of gamma-ray codes known as $\mathrm{G}^{3}$, which borrow the very versatile combinatorial geometry routine from QAD (Ma73). The $\mathrm{G}^{3}$ codes can group detector respouses by source energy or scattered gamma energy, and can report doubly differential flux densities by energy and direction of the incoming photons.

$\mathrm{G}^{3}$ employs the point source and point detector geometry first used by Trubey, with many of the conventions of single-scatter calculations. At each scattering point between source and detector, the uncollided flux density is 
multiplied by the probability of a Compton scatter toward the detector for a photon of the source energy. Treating the scattering point as a new source, the uncollided flux density at the detector due to the "scattering source" is calculated and multiplied by the buildup factor in infinite air for the scattered photon energy. In running $\mathrm{G}^{3}$, the user defines scattering "boxes;" for simplicity, scatters occur only at the center of each box, and resulting quantities are integrated over the volume of the box.

One sample problem used to test $\mathrm{G}^{3}$ is the same line-beam problem studied by Lynch et al., Trubey and Kitazume. In comparison to the Monte Carlo results of Lynch et al., $\mathrm{G}^{3}$ gives results within $10 \%$ for most distances, except at the shortest source-detector distances. (RS85) There are problems which $\mathrm{G}^{3}$ cannot address well, however; one of these is discussed in the next section. Still, $\mathrm{G}^{3}$ is a useful and reliable code in some skyshine applications, and it will be used for comparisons in this work.

\subsection{Other Numerical Skyshine Studies}

A study recently completed at Kansas State University (Fa87) employs a method different from both Monte Carlo and single-scatter techniques. The MicroSkyshine method was first based upon the results of Lampley's SKYSHINE-II line-beam Monte Carlo code (La79), and the first MicroSkyshine code incorporated a data base from SKYSHINE-II. The data base contained coefficients of an empirical equation which had been fit to Monte Carlo results; the MicroSkyshine program interpolated values of these coefficients to yield gamma-ray response functions continuous over source energy and direction. 
The method's simplicity makes it suitable for use on microcomputers, and MicroSkyshine is now commercially available. This version solves problems involving a gamma-ray point source inside a cylindrical silo, or a point, line or plane source of photons behind a semi-infinite wall. Slab shields may be placed above the source, over the silo or wall. Such simple geometries might be used as approximations of more complex real situations, such as a ${ }^{16} \mathrm{~N}$ gamma-ray source within a turbine building.

The first version of MicroSkyshine used photon response functions from SKYSHINE-II; the code was validated against two ANSI-standard reference problems (AN87) and $\mathrm{G}^{3}$ results for the silo and wall geometries. For the ANSI problems, the MicroSkyshine geometries were adapted as closely as possible to the reference problems, though exact replication was not possible. The results from the code and the ANSI standard were in very close agreement. When compared to MicroSkyshine values for the simple geometries, $\mathrm{G}^{3}$ values were consistently lower in cases with a slab shield over the source. A comparison of both codes' results to benchmark experimental data discussed in the next section (Ro80) revealed that $\mathrm{G}^{3}$ seriously underpredicts in those instances because it cannot account for gamma-ray scattering and buildup in the slab. The MicroSkyshine responses proved more accurate, validating the method in these cases. Overall, Faw and Shultis report the method results are conservative and within $50 \%$ of nearly all documented reference values. (Fa87)

The version of MicroSkyshine available to the public uses an improved set of response functions developed specifically for the method by Shultis and Faw (Sh87). The original response functions were developed by Radiation Research Associates for SKYSHINE-II. The empirical equation for response had been fit to results of 
Monte Carlo computations, and the parameters contained small discontinuities for adjacent energy groups and directions. The errors, probably from statistical variations, propagated into the computed skyshine dose of early versions of MicroSkyshine. The old response functions also led to overprediction of dose for large source-detector distances, and extrapolation for high energy photons and large distances could give negative response values.

The new gamma-ray response functions were computed by a point-kernel formula much like the line-beam dose formula of Kitazume (Ki6s), but which also included a response from annihilation photons created by pair-production positrons in air. The integration required to arrive at the response functions was accomplished numerically with Gaussian quadrature. To describe the functions, let $\mathscr{R}(E, \mathrm{x}, \phi)$ be the dose at distance $\mathrm{x}$ from the source, in air of standard density $\rho_{0}$, resulting from a photon of energy $E$ emitted at an angle $\phi$ from an axis between the source and detector. These responses, computed by the point-kernel formula, were fitted to the approximating function

$$
\mathscr{R}(\mathrm{E}, \mathrm{x}, \phi)=\kappa \mathrm{E}\left(\rho / \rho_{0}\right)^{2}\left[\mathrm{x}\left(\rho / \rho_{0}\right)\right]^{\mathrm{b}} \exp \left[\mathrm{a}-\mathcal{C x}\left(\rho / \rho_{0}\right)\right],
$$

where $\kappa$ is a constant conversion factor, $\rho$ is the air density, and a, b, and $\mathrm{c}$ are empirical parameters.

Response functions were computed at discrete source energies and beam directions from the source-detector axis, then fit to this formula, the same empirical formula as was used with the original Monte Carlo functions. With linear interpolation, however, the new parameters produce no discontinuities or negative values, and the new response functions are accurate for source-detector distances up 
to 5000 meters compared with 1500 meters for the SKYSHINE-II response functions. Besides clearing up the difficulties mentioned in the previous paragraph, the new photon response functions eliminate small variations in dose as the system geometry is changed slightly. Most important, comparison with benchmarks shows the new response functions produce more accurate and realistic results and are in better agreement with benchmark data than the old results. (Sh87)

\subsection{Experimental Work}

Although the early paper by Zerby (Ze56), discussed at the beginning of this chapter, described computations made on experimental measurements of neutron radiation in air, it does not describe the experiments thoroughly. To this author's knowledge, only one benchmark skyshine experiment involving point sources has been documented in detail. This gamma-ray skyshine experiment was performed in 1979 at the Kansas State University Nuclear Engineering Shielding Facility, sponsored by the Japanese Nuclear Safety Research Association, and documented in an article by Nason et al. (Na81).

In the experiment, three ${ }^{60} \mathrm{Co}$ point sources of strength ranging from $10 \mathrm{Ci}$ to $3800 \mathrm{Ci}$ were placed at the axis of a cylindrical concrete silo. A high pressure ionization chamber for exposure rate measurements, and a sodium iodide detector for photon energy spectra measurements, were placed at distances up to $700 \mathrm{~m}$ from the source. The three source configurations included a collimator on the open silo to direct the gammas into a 150-degree cone, and concrete shields of thickness $21 \mathrm{~cm}$ or $42.8 \mathrm{~cm}$ over the silo. The thickness of the silo walls prevented much radiation from leaving the silo horizontally and contributing to measured exposure rates. Also, a lead and concrete collimator on the NaI assembly removed background 
radiation from the measured spectra. Both of these features, along with background measurements, allowed the dose and spectra due solely to gamma-ray skyshine to be determined easily.

Results from both detector systems were corrected for variations in response with the energy of the incident radiation. These corrections came from calibration tests and manufacturer's data on the instruments. For comparison purposes, calculations were performed using DOT, a two-dimensional discrete ordinates transport code; tbe cylindrical silo geometry was selected for its reproducibility with such programs. As a test of the accuracy of the results from both detectors, exposure rates were computed from the $\mathrm{NaI}$ spectral measurements and corrected from the collimation angle on the detector to a $4 \pi$ exposure rate. For nearly all experimental cases, these exposure rates agreed very well with those from the ionization chamber. Thus, the experimental results can be used reliably as a benchmark for predictive methods and as design data for nuclear facilities. (Na,81)

One predictive method which has been tested against these benchmark results was the subject of a thesis by Roseberry (Ro80). The method uses a variation of the infinite air, single-scatter equation of Kitazume (Ki68), but as Faw, Roseberry and Shultis point out (Fa86), the addition of the concrete slabs over the silo to the model and the treatment of the scattering angle as an independent variable are significant improvements. Roseberry's results compare favorably with 1979 ANSI-standard calculations and the benchmark experiment data, although no ground-air interface is used and the model overpredicts tbe experimental results. Roseberry's method also yields more accurate results than DOT when the silo is covered by a concrete roof. This example may encourage the use of the benchmark experimental results to test other programs or designs, since Roseberry easily 
modeled the cylindrically symmetric geometry. Roseberry's model is discussed in detail in Chapter 3 of this work.

Although experiments to measure skyshine exposure rates are rare, measurements are often taken in practical situations where direct and scattered gamma rays are present. In the case of light water nuclear reactors, the direct and scattered photons from contained radionuclides is one of the many components of the radiation field on site. To measure these gamma rays, their contribution to the overall response must be isolated. Time dependence must also be taken into account. An American National Standard was developed for the calculation and measurement of such gamma radiation from LWRs in the mid 1970's, and a revised Standard was issued in 1987. This standard describes what factors are important in designing a radiation measurement program, the tools available to measure radiation fields, the techniques of measurement, and how data from them should be interpreted in light of their limitations. (AN87) These recommendations might prove useful in future skyshine experiments with regard to experimental setup and data interpretation, should a benchmark experiment be contemplated. 


\subsection{COMPUTATION OF SKYSIIINE EXPOSURE RATES FOR SIMPLE GEOMETRIES}

As mentioned in Chapter 2, numerical computation of radiation fields and doses can be less costly and time consuming than physical modeling with cxpcrimental radiation sources. Of the two common numerical schemes that were revicwed, the single-scatter and buildup model normally requires less time and effort to program and execute. In this chaptcr, the interactions and assumptions which make up the single-scatter and buildup model are explained. Next, a gencral exposure rate equation is presented for the case of a point source and point detector in air. This equation uses the single-scatter and buildup model, and was first presented by Rosebcrry (Ros0) in his thesis. The remainder of the chapter is devoted to the description of two simple geometries for which the photon exposures are calculated by this point-kernel cquation. One gcometry involves a point gamma-ray source inside an open, cylindrical silo, with a point detector some distance away outside the silo. The other gcometry separates the source and detector by a wall extending to infinity on the left, right and bottom, but having a top edge. In cither geonctry, a concrete roof slab may be placed over the source.

The usefulness of a model involving point sources may secm limited for practical cascs involving, for instance, steam lines. However, point sources in modeling lave been endorsed in the American National Standard ANSI/ANS-6.6.1-1987. In the modeling of BWR turbine buildings, the Standard states that the source need not be detailed, and equivalent point sources may be used, if desired, to estimate dose rate. Source strengths may be taken from plant specifications; self-shielding of the source may also be uscd, but should be cxplained or discussed if it is used. (AN87) All these 
points would appear to support the substitution of a point source for more complex sources in numerical models.

The ANSI standard is also clear on the use of shielding and the modeling of skyshine: "All significant sources of gamma radiation...which are essentially unshielded relative to air scattering (i.c., shielded on the sides but essentially unshielded on the top) shall be considered. Sources which are shielded only by the outside shicld wall of a building should be considered.... All sources of gamma radiation which are located outside of shielded buildings shall be considered." (AN87) These three situations correspond to the silo geometry, the wall geometry, and the problem of a point source in all infinite air medium, respectively. The standard also requires that shielding in any form must be represented by the model, positioned and oriented correctly with respect to the source or sources, witlin the limitations of the numerical method used. Topography, such as bluffs or buildings acting as shadow slields, should also be considered, but are not required in the model. (ANS7) The two geometries studied in this work were chosen with the American National Standard in mind, and can meet several of its requirements, including those of slijelding and (for the wall problem) topograplyy.

\subsection{The Single-Seatter and Buildup Model}

The model used by both computer codes written for this study is the single-scatter and buildup model for gamma rays. The method is normally applied to situations involving point photon sources and point detectors in air. It can be extended to problems of line, plane and volume sources, but only with a dramatic increase in computational effort, and no such extension will be 
discussed. Similarly, other media might be used, but the method was developed solely for air skyshine studies, so only air will be the primary medium.

Consider a photon leaving a point source and traveling in some arbitrary direction through an infinite air medium. After traveling some distance, the photon will suffer an interaction with the air, resulting in one or more photons or electrons of different direction and energy than the source photon. The actual result depends on the source photon energy and the energy-dependent, angular-dependent cross sections of air. The volume of air where the photon interacts may be thought of as a "first-collision source" of photons and electrons; in the case of gamma-ray skyshine, only photons are of concern.

The gamma rays which reach the point detector may have scattered once, more than once, or not at all after leaving the source. It would require the resources of a Monte Carlo code to trace every photon through every interaction, and not every gamma ray followed in this manner would reach the detector. Instead, the photon flux density at the detector can be found analytically by treating the first-collision source as a point source of secondary gamma rays, as the name implies. The energy and iutensity of the photons leaving the first-collision source will depend on the direction, energy and intensity of the gamma rays from the true source, and the cross-sections for ganma rays in air of interactions which create or scatter photons.

By applying inverse-square attenuation and exponential attenuatiou, the uncollided flux density at the detector from the first-collision source can be found. By also applying a buildup factor for a point source in infinite air, the total flux density at the detector from the single-scatter source can be 
estimated. In a sense, all photons which scatter for thc first time at the volume of interest and evcntually reach the detector are collected together and computed in one step using buildup, hence the name for the method.

Figure 3-1 shows a simple illustration of the model for a particular scattering volume. The first $\operatorname{leg}$ of the gamma-ray journey involves only attenuation of photons traveling to the first-collision source; attenuation and buildup are applied on the sccond "leg" of thc journey, since not all photons follow this path preciscly. If the flux densitics or responses at the detector due to all scattering volumes are added together, the rcsult is an estimatc of the total scaltered flux density or detector response. This summation may be accomplished by expressing the quantity of interest as a function of position of the scattering volume, and integrating the function over all spacc for which first-collision sources bave a clcar path to the point detector. The total response at the detector is approximately the sum of tbe uncollided response from the point source and this first-collision integral.

The intcractions considercd at the first-collision source and the application of buildup deserve much attention, and will now be discussed in detail.

\subsubsection{Interactions Considered}

Gamma rays undergo many kinds of intcractions in a typical medium. The threc most common intcractions, of course, are Compton scattering, photoelectric interactions, and pair production. Because gamma-ray skyshine is not concerned witb elcctrons, photoelectric effects are gencrally neglected; in these cases, the gamma ray is absorbed entirely and lost at the collision point, 


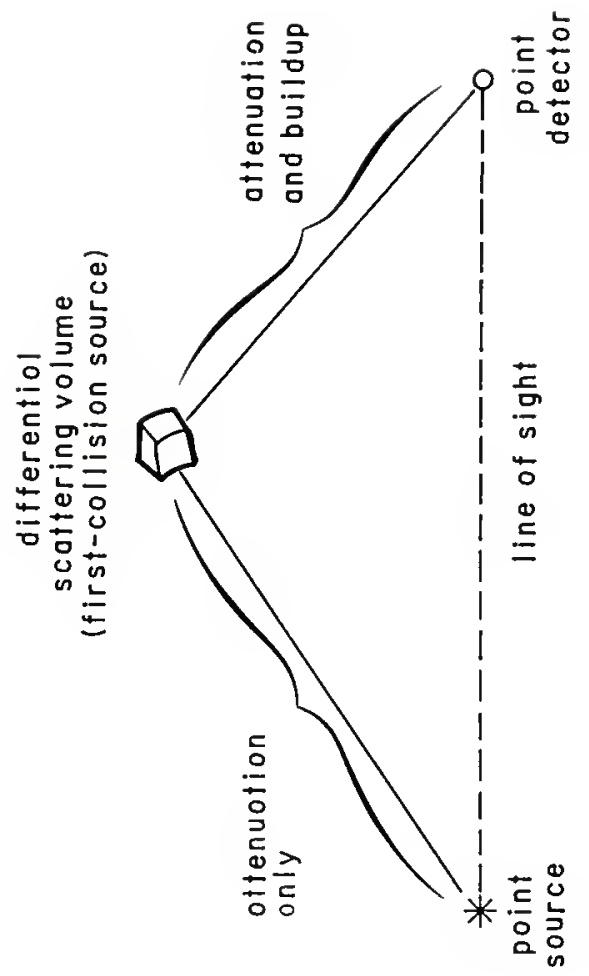

吾

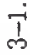

E 
producing a frce electron. The ZAP code (Ma69) did consider photoelectric effects for X-rays, but it was necessary to track the electrons to their absorption points to provide the fluorescent light source for the PFLASHl and FLASH codes (Co69). ZAP may be considered an exception to the rule.

The remaining interactions are now discussed in the context of ganma-ray analysis.

Compton Scattering. The Compton interaction has been used in every application of the single-scatter and buildup model that the author has reviewed, and should be considered a requirement of the method. Compton scattering depends directly and simply on the position of the first-collision source through the Compton formula for scattered photon energy and the Klein-Nishina cross section. Also, for photons of energies encountered in common situations (50 keV to $10 \mathrm{MeV}$, for instance), Compton scattering dominates interactions in air by a factor of two or more. This can be seen in Fig. 3.2, where intcraction coefficients in air are graphed for the three primary interactions. The early Monte Carlo skyshine study by Lynch et al. (Ly58) involved only Compton scattering; the authors stated that for their interests, photoelectric interactions were of no use, and all other primary and sccondary processes were negligible by comparison.

The use of the Compton and Klein-Nishina formulas in the model requires an approximation that free electrons and elcctrons bound to atoms possess the same scattering characteristics. This is because the Klein-Nishina. formula is correct strictly for free electrons, electrous not residing in an atomic orbital. The RRA code ZAP did not make this approximation, and corrected 


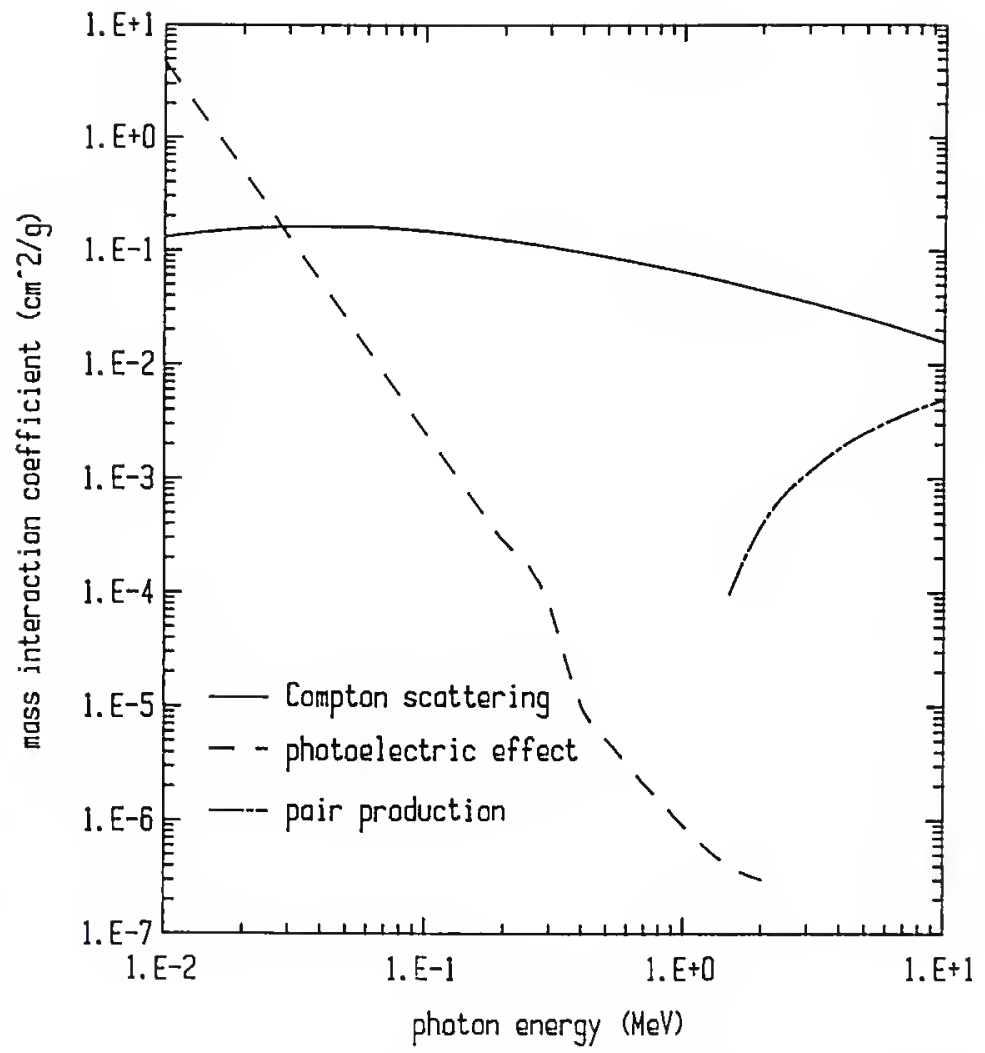

Fig. 3-2. Mass interaction coefficients for gamma rays in air. Data from (Ch84). 
the Compton ilsteraction coefficients for electron binding effects (Ma69). Chilton et al. state that such a correction is not necessary, and the approximation that all electrons are unbound is quite valid. They point out that the Klein-Nishina formula fails when the kinetic energy of the recoil electron approaches its binding encrgy, a situation found only with low photon energies and material of high atomic number. At such low energies, however, the photon is much morc likely to undergo a photoclectric interaction than incoherent scattering, and the error due to the approximation is negligible. (Cli84) Also, air contains only negligible amounts of high-Z materials, so recoil electrons in air are not likely to have kinetic energies comparable to their binding energy. With these facts in mind, the approximation of free electrons is quite justified, and is used in this work.

Pair Production. The photons resulting from Compton interactions are emitted from the point of collision, as are the recoil electrons. In the case of pair production, however, an clectron and a positron are emitted from the collision point; the positron travels a distance before encountering an elcctron and annihilating. Two $0.511 \mathrm{MeV}$ gamma rays are emitted from this point, and it is these annihilation quanta which are of interest in gamma-ray analysis.

The choice of whether or not to include the annihilation photons was first based upon the distance traveled by the positron. For instance, the Monte Carlo code ZAP included pair production in its calculations. Marshall et al. stated that pair production positrons are likely to travel no more than three meters in air before they interact with an electron. Since the distances 
involved in the study were comparable to the earth's radius, the annihilation was assumed to occur at the site of pair production, with the two photons emitted back-to-back and isotropically. (Ma69) When pair production is included in modern codes, the same assumption is made; the MicroSkyshine code also neglects the distance traveled by the positron in order to include the annihilation photons in its point-kernel computations (Sh87).

A second and more common consideration is the energy of the source photons. Pair production has a minimum gamma-ray cutoff energy of 1.02 $\mathrm{MeV}$; photons below this energy cannot induce pair production. The interaction coefficient is negligible for gamma-ray energies below $1.5 \mathrm{MeV}$, and is still a full order of magnitude below the Compton interaction coefficient for photon energies below $5 \mathrm{MeV}$ (Ch84), as can be seen in Fig. 3-2. For situations involving ${ }^{16} \mathrm{~N}$ sources, which most often emit $6.13 \mathrm{MeV}$ gamma rays, pair production can make significant contributions to skyshine. Otleer sources are not as likely to give rise to annihilation photons in this manner.

For this work, it has been decided to neglect pair production effects. The criterion used to justify pair production in ZAP is not the reason; those few codes which include the process always ignore the distance traveled by positrons. Few of the numerical methods reviewed in Chapter 2 or used for comparisons later in this work account for pair production, so including the process here would make the comparisons difficult. Although the codes will underestimate detector responses that would occur in reality, it is necessary that these comparisous be made without pair production to validate the method. Pair production can easily be included in the programs if deemed necessary. 
Other Intcractions. Any other interactions which may produce photons or scatter gamma rays are customarily ignored. For instance, as elcctrons are ejected from a nucleus by photoelectric interaction, they leave behind an excited atom which may emit fluorescence photons. The energy of these photons is never higher than the binding energy of the photoelectron. For air, tle fluorescence is in the $\mathrm{eV}$ range, far too low in energy to be of interest. Coherent (Rayleigh) scattering has a negligible effect on the energy or direction of the photon, and it too is ignored in most shielding work. (Ch84) The Monte Carlo code FLASIl did apply Rayleigh scattering, but was concerned with visible fluorescent light. For such low-cnergy photons, Rayleigh scattering is significant, as are unusual processes such as refraction by air molecules and scattering and absorption by aerosol particles (Co69). Indeed, FLASH incorporated many processes which apply only to visible light and have no use in gamma-ray skyshine analysis.

Evaluation of intcraction cocfficients. From the discussion above, it is clear that only Compton interactions are regularly used in single-scatter and buildup models. Therefore, the Klein-Nishina formula is often the only interaction coefficient evaluated, and it may easily be computed for a given gamma-ray energy. Where pair production is included, tabulated coefficients have been used (Ma69, Sh87), and interpolation would be necessary for photon energies not in the tables. This would slow down evaluation of the firstcollision source in some cases.

For situations where no concrete shielding is placed over the point source, evaluation of the cross sections is simple. Because the photons reaching the scattering volune (or first-collision source) have their first 
interaction at that point, the cross section is taken at the energy of the source photons. In cases where shielding covers the gamma source, however, it may be difficult but sound practice to find the spectrum of uncollided and collided photons leaving the shield in the direction of the first-collision volume, then apply a spectrum-wcighted cross section at the volume. Roscberry and Shultis (Ro82) argue that this is not necessary. They state that photons which leave a thick slab shield are essentially collimated into a beam of uncollided photons or photons which liave undergone small deflections and are nearly unchanged in energy. Any photons which have suffered collisions and lost energy in the slab will be attenuated by the atmosphere and can be neglected when computing exposure rates far from the point source. Roseberry and Shultis conclude that the Compton scattcring cross section still may be evaluated at the source energy when a concrete shicld is present above the source. This assumption reduces computational effort required by the model and will be applied in this thesis.

ln summary, Compton scattering is the only interaction regularly used in the single-scatter and buildup model, although some works have included pair production. This study will consider only Compton scattering at the first-collision source.

\subsubsection{Application of Buildup}

To account for photons whicl scatter more than once, a buildup factor is applied to the second leg of the gamma-ray path showu in Fig. 3-1. Buildup has also been employed in a limited fashion in shields over the point source. 
The use of buildup factors requires some assumptions, which will be discussed here.

Buildup factors commonly available for computations are for point isotropic sources in infinite media. In some simulations, such as that used by Roseberry for the point source in a silo, the air-ground interface is neglected and an infinite air medium is used instead. This justifies the use of an infinite-air buildup factor after one scatter, but the approximation results in overprediction of exposure rates near the ground, since earth tends to absorb more scattered photons than air. On the other hand, the first-collision source at the scattering volume is not isotropic, since Compton scattering is biased in the forward direction. (Fa86) This makes the use of a buildup factor derived from an isotropic source inappropriate. Still, Shultis and Faw demonstrated that applying the isotropic buildup factor to the non-isotropic scattering source introduces only a small error to the results of MicroSkyshine (Sh87). It may be that this practice in Microskyshine underpredicts the detector response, compensating for the overprediction from the use of infinite air instead of an eartli-air interface.

When the point source in Fig. 3-1 is collimated upwards but open to the air, the first-collision volume can correctly be treated as the point of first interaction for the gamma ray. All buildup can be applied to the second leg of the photon path, and no buildup need be applied to the path between source and scattering volume. Roseberry and Sluultis proved that the single buildup factor provided the best results in a point-kernel code, using benclimark measurements for comparison. (Ro82) If a concrete slab or other shield covers the collimated source, as in Fig. 3-3, scattering will occur in tbe 


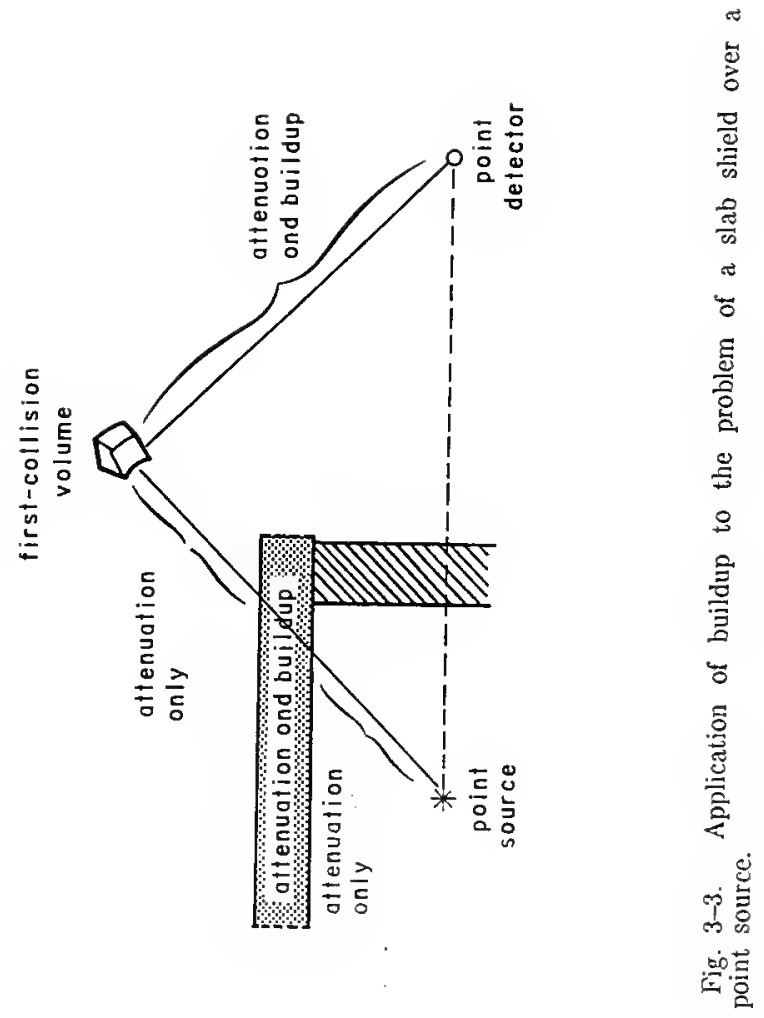


shield and should be accounted for in some manner. The approach taken by Roseberry and Shultis is to apply an infinite-medium buildup factor for a point isotropic source in concrete. The factor is applied over the appropriate distance through the slab shield, and is evaluated at the gamma-ray source energy. Further, it is assumed in this approach that all photons leaving the shield, including those counted in buildup, remain at the source energy. While the last approximation is not correct, it allows cross sections at the first-collision source to be evaluated only at the source energy, not several energies. Roseberry and Shultis show that the use of the second buildup factor in the concrete shield yields far more accurate results than the omission of buildup in concrete entirely. Although its use is a rough approximation, it is "extremely important." (Ro82) Faw and Shultis (Fa87) later stated that the concrete buildup approximation, combined with attenuation through the concrete, gave reasonable values of point exposure rates for shield thicknesses of six mean free paths or less. They could not validate the approximation for larger shields, however. The approach is studied in detail by Bassett (Bas8).

In summary, to use buildup factors in air between the first-collision source and the detector, the air-ground interface should be approximated by infinite air, and the first-collision source should be treated as a point source with a source strength per steradian equal to the intensity leaving the differential volume in the direction of the point detector. In cases where a concrete shield covers the point source, buildup factors may be used in the concrete, treating all photons entering and leaving the shield as photons at the source energy. The shield should be of moderate thickness, only a few mean free paths if possible. 


\subsection{The Exposure Rate Integral Equation}

With the assumptions of the single-scatter and buildup model outlined above, an equation for the exposure rate at the detector may now be presented. Exposure is the response commonly reported when only gamma-ray sources are present. Formulation of the model was introduced by Kitazume (Ki6s) to evaluate dose rate, with a generic factor to convert flux density to the desired response, and with the Taylor buildup approximation incorporated into the formula. Similar analytical equations have been presented in descriptions of FLASH (Co69) and $\mathrm{G}^{3}$ (Ma73). The derivation for $\mathrm{G}^{3}$ is notably straightforward, and very much like the derivation to follow, although $G^{3}$ converts the first-collision source to an equivalent isotropic source.

The exposure rate equation presented here was derived by Roseberry (Ros0) and repeated without proof in the paper of Roseberry and Shultis (Ro82); in the former reference, it is shown to be equivalent to Kitazume's formula. The formula is derived here in a brief manner; the reader should consult the thesis of Roseberry for more detail.

The infinite-air, single-scatter geometries of Fig. 3-1 and Fig. 3-3 are repeated in Fig. 3-4, with geometric distances labeled. The source and detector are separated by a distance $\mathrm{d}$ along the line of sight; this line will be referred to as the source-detector axis. Photons leaving the source travel a distance $a$ through air to reach the first-collision volume, and if a concrete slab is present, also travel a distance $a_{c}$ through the concrete. The total distance from the source to the differential first-collision volume $\mathrm{dV}$ is labeled $\overline{\mathrm{a}}$; the point detector lies at distance $\mathrm{b}$ from $\mathrm{dV}$. 


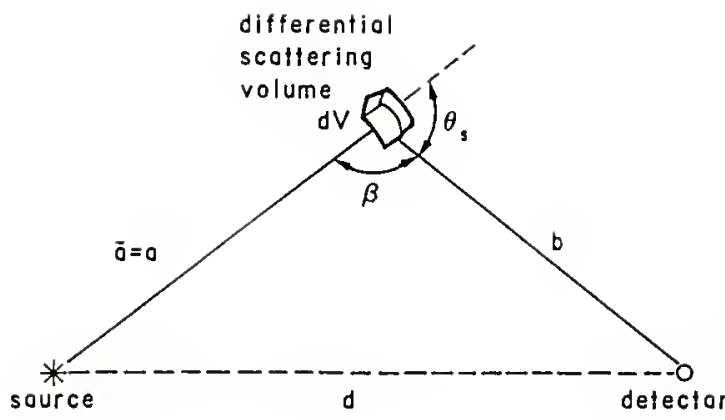

(a)

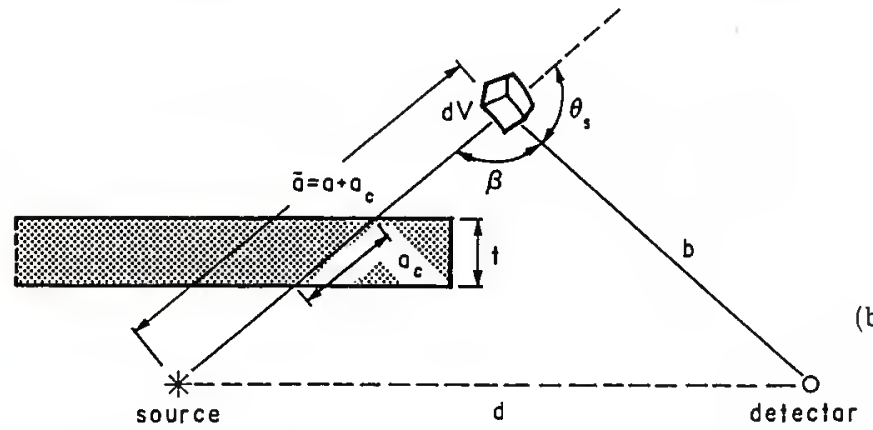

(b)

Fig. 3-4. Distances involved in the single-scatter geometry: overhead shield; (b) overhead concrete roof present. 
In the absence of a concrete roof, the uncollided gamma-ray flux density at the first-collision volume $\mathrm{dV}$ is given by the simple formula

$$
\phi^{0}=\frac{\mathrm{S}}{4 \pi \mathrm{a}^{2}} \exp (-\mu \mathrm{a}),
$$

where $\mathrm{S}=$ source strength,

$\mu=$ linear attenuation coefficient in air for source photons of energy E.

If a concrete slab is placed over the source, source photons will also be collimated by the material of the slab. The uncollided gamma-ray flux density at $d V$ is now

$$
\phi^{0}=\frac{\mathrm{S}}{4 \pi\left(\mathrm{a}+\mathrm{a}_{\mathrm{c}}\right)^{2}} \exp \left(-\mu \mathrm{a}-\mu_{\mathrm{c}} \mathrm{a}_{\mathrm{c}}\right)
$$

where $\mu_{\mathrm{c}}=$ linear attenuation coefficient in the roof material for source photons of energy $\mathrm{E}$.

Recall the assumption that photons reaching the first-collision volume $d V$ have their first interaction in that volume. As long as this assumption holds, only uncollided flux density need be used to compute interactions in dV. Also, recall from Section 3.1.1 that only Compton scattering will be considered in this work, so the first-collision volume may be properly called a "differential scattering volume," a more common name in the literature.

The number of photons which reach the differential scattering volume $d V$ and scatter through an angle $\theta_{\mathrm{S}}$ (see Fig. 3-4) into a solid angle of unit steradian toward the detector may be written as

$$
\mathrm{S}_{V}=\phi^{0 \mathrm{~N} \sigma \mathrm{d} V}=\frac{\mathrm{SN} \sigma}{4 \pi\left(\mathrm{a}+\mathrm{a}_{\mathrm{c}}\right)^{2}} \exp \left(-\mu \mathrm{a}-\mu_{\mathrm{c}} \mathrm{a}_{\mathrm{c}}\right) \mathrm{dV},
$$


where $N=$ electron density in $\mathrm{dV}($ Ros0)

$$
\begin{aligned}
& =\left(3.0064 \times 10^{23} \mathrm{~g}^{-1}\right) \rho_{\text {air }}, \\
\text { and } \sigma & =\text { differential Compton scattering cross section } \\
& =\sigma\left(\theta_{\mathrm{s}}, \mathrm{E}\right) .
\end{aligned}
$$

The energy of the photon after scattering through an angle $\theta_{S}$ depends upon $\theta_{S}$ and the initial photon energy E. Using the approximation of free electron interactions for bound electrons in air, the energy $\hat{E}$ of a scattered photon can be related to its initial energy $E$ and the complement $\beta$ of the scattering angle $\theta_{\mathrm{s}}$ by the Compton formula (Ch84),

$$
\hat{E}=\frac{E}{1+\frac{E}{m_{e} c^{2}}(1+\cos \beta)},
$$

where $\mathrm{me}_{\mathrm{e}} \mathrm{c}^{2}$ is the rest mass energy of an electron. The supplement of the scattering angle was chosen here, since $\beta$ will be used later as an independent variable. The cross section itself can be evaluated with the Klein-Nishina differential scattering formula (Ch84), which appears in dimensional form in (Ros0) as

$$
\sigma=\frac{\mathrm{r}_{\mathrm{e}}^{2}}{2} \frac{\lambda^{2}}{(1+\lambda+\cos \beta)^{2}}\left[\frac{\lambda}{1+\lambda+\cos \beta}+\frac{1+\lambda+\cos \beta}{\lambda}-\sin ^{2} \beta\right],
$$

where $r_{e}=$ classical electron radius (Ch84)

$$
=2.818 \times 10^{-15} \mathrm{~m},
$$

and $\lambda=$ Compton wavelength

$$
=m_{e} c^{2} / E \text {. }
$$


The formula is evaluated at the source energy, since it is assumed that all gamma rays reaching the differential scattering volume are uncollided or have lost no energy.

Next, the differential exposure rate $d \dot{X}_{S}$ at the detector due to photons scattering only at $\mathrm{dV}$ in a steradian toward the detector may be written as

$$
d \dot{X}_{s}=\frac{S_{V}}{b^{2}} \frac{\hat{\mu}_{e n}}{\rho} \hat{E} K \exp (-\mu b)
$$

where $b=$ distance in air from the scattering volume to the detector,

$$
\begin{aligned}
\frac{\hat{\mu}_{\mathrm{en}}}{\rho}= & \text { mass energy absorption coefficient in air for } \\
& \text { photons of energy } \hat{\mathbf{E}}, \\
\hat{\mu}= & \text { linear attenuation coefficient in air for ganma } \\
& \text { photons of energy } \hat{\mathrm{E}}, \\
\mathrm{K}= & \text { energy flux-to-exposure-rate conversion factor (Ch84) } \\
= & 1.835 \times 10^{-8} \mathrm{R} \cdot \mathrm{g} \cdot \mathrm{MeV}-1 .
\end{aligned}
$$

Substituting for $S_{v}$ from equation (3-3) produces

$$
\mathrm{d} \dot{X}_{\mathrm{S}}=\frac{\mathrm{SKN} \sigma}{4 \pi\left(\mathrm{a}+\mathrm{a}_{\mathrm{c}}\right)^{2} \mathrm{~b}^{2}} \frac{\hat{\mu}_{\mathrm{en}}}{\rho} \hat{\mathrm{E}} \exp \left(-\mu \mathrm{a}-\mu_{\mathrm{c}} \mathrm{a}_{\mathrm{c}}-\hat{\mu b}\right) \mathrm{dV} .
$$

This is the uncollided exposure rate at the detector from the first-collision source; gamma rays which scatter more than once must be accounted for using buildup factors as discussed in Section 3.1.2.

A buildup factor $\hat{B}$ accounts for multiple scattering along the path from the scattering volume $d V$ to the detector. This factor is evaluated at the 
scattered gamma-ray energy $\hat{\mathrm{E}}$ and over the distance $\mathrm{b}$; the scattering volume is treated as a first-collision source of photons of energy $\hat{\mathrm{E}}$, located a distance b from the detector. Another buildup factor $\mathrm{B}$ accounts for gamma-ray scattering in the concrete roof, if one is present. This factor is evaluated at the source energy $E$ and applied over the concrete path length $a_{c}$; photons leaving the slab are assumed to retain their energy. The assumption that $\mathrm{dV}$ is the point of first interaction fails when the concrete is involved; the buildup factor $B$ increases the flux density at the scattering volume, since it is not solely given by Eq. (3-2).

Correcting the single-scatter exposure rate equation for buildup produces a new differential exposure rate expression, Eq. (3-8),

$$
\mathrm{d} \hat{\mathrm{X}}_{\mathrm{s}}=\frac{\mathrm{SKNB \hat {B } \sigma}}{4 \pi\left(\mathrm{a}+\mathrm{a}_{\mathrm{c}}\right)^{2} \mathrm{~b}^{2}} \frac{\hat{\mu}_{\mathrm{en}}}{\rho} \hat{\mathrm{E}} \exp \left(-\mu \mathrm{a}-\mu_{\mathrm{c}} \mathrm{a}_{\mathrm{c}}-\hat{\mu} \mathrm{b}\right) \mathrm{dV}
$$

Integration of Eq. (3-8) over all space $\mathrm{V}$ for which photons may scatter once and travel from source to detector gives the final expression for the total exposure rate $\dot{\mathrm{X}}$ at the detector,

$$
\dot{\mathrm{X}}=\int_{V} \frac{\mathrm{SKNB} \hat{\mathrm{B}} \sigma}{4 \pi\left(\mathrm{a}+\mathrm{a}_{\mathrm{c}}\right)^{2} \mathrm{~b}^{2}} \frac{\hat{\mu}_{\mathrm{en}}}{\rho} \hat{\mathrm{E}} \exp \left(-\mu \mathrm{a}-\mu_{\mathrm{c}} \mathrm{a}_{\mathrm{c}}-\hat{\mu} \mathrm{b}\right) \mathrm{dV} .
$$

This formula includes attenuation and buildup in a concrete roof over the point gamma-ray source. For cases where the roof is absent, Eq. (3-9) holds with $\mathrm{a}_{\mathrm{c}}=0$ and $\mathrm{B}=1$.

The space $V$ over which integration takes place is dependent upon the physical problem, whether a silo or a wall is present, for example. Selection 
of proper limits of integration will allow the application of Eq. (3-9) to many shielding situations. A good choice of coordinate system will also make evaluation of the integral equation easier. Both of these issues are addressed in the remainder of the chapter. Section 3.3 applies Eq. (3-9) to the case of a point source in a cylindrical silo; section 3.4 considers a point source behind a semi-infinite wall.

\subsection{Application of the Exposure Rate Integral Equation to a Silo Geometry} The prohlem of a point source of gamma rays within a cylindrical silo has been solved numerically by Roseherry (Ros0), and reported again hy Roseberry and Shultis (Ros2). Their coordinate system and limits of integration will be repeated here, and improvements made to the computer code used by Roseberry will he examined.

The problem is illustrated in simplified fashion in Fig. 3-5. A point source of gamma rays is located at the axis of a cylindrical silo with perfectly absorbing walls. The top of the silo is open to the air and collimates the gamma rays vertically into a known solid angle. Alternately, a concrete slab of thickness $t$ is placed over the silo and point source. A point detector is located at distance $d$ from the point source, at the same elevation as the source. The source and detector are helow the top of the silo, so that photons must scatter to reach the detector. In hoth cases, the medium is simulated as infinite air; no ground interface is used in calculations. 

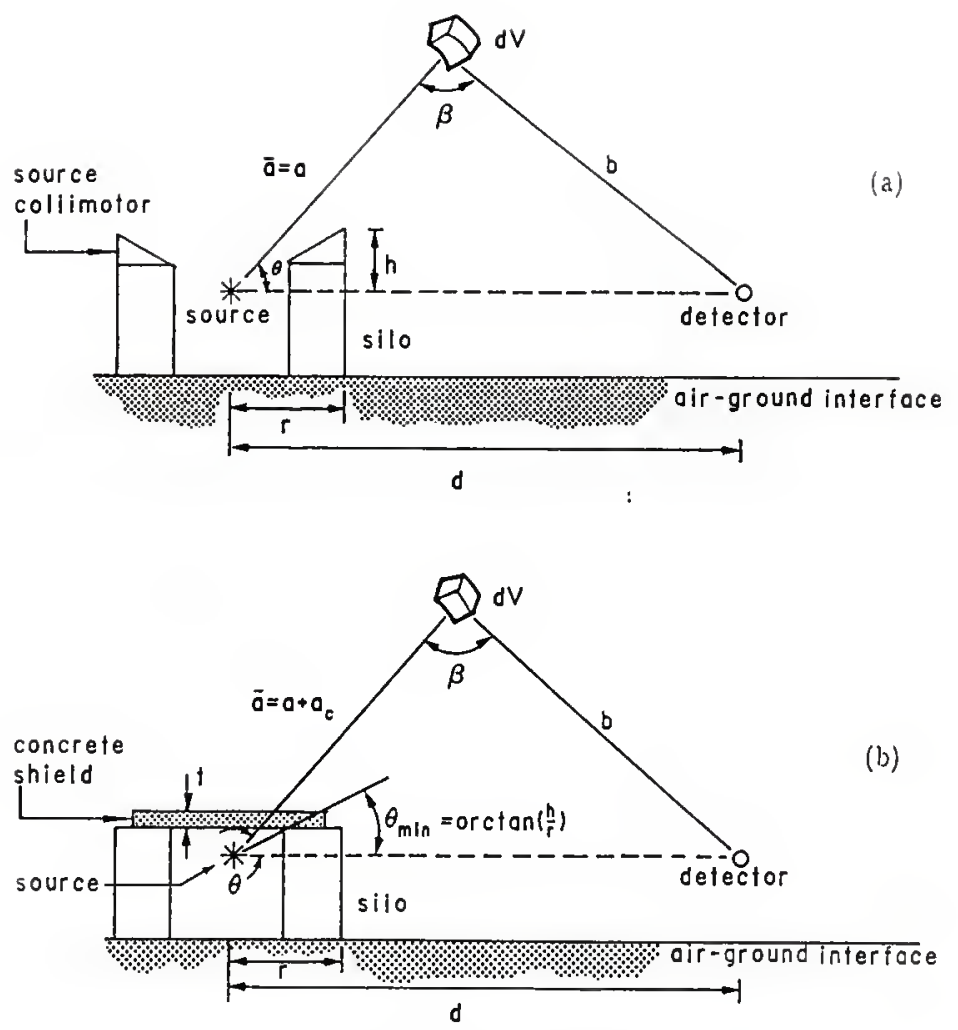

Fig. 3-5. Simplified calculational geometry for the case of a point source within a silo: (a) no overhead shield; (b) overhead concrete roof present. [From (Roso)] 


\subsubsection{Coordinate System}

To integrate Eq. (3-9) over al\$ space in which an uncollided gamma ray may scatter once and reach the detector directly, a three-dimensional coordinate system must be selected. For the silo problem, the coordinate system consists of three angles $\beta, \theta$, and $\epsilon$, defined in Fig. 3-6. The angles $\theta$ and $\epsilon$ specify the direction in which a photon leaves the source, while $\beta$ locates the scattering volume $d V$ along that path. In this system, the variables $\sigma, \hat{\mathrm{E}}$ and $\hat{\mu}_{\mathrm{en}} / \rho$ depend only on $\beta$. Using this coordinate system yields the following expressions (Ros0, Ro82):

$$
\begin{aligned}
\mathrm{b} & =\mathrm{d} \sin \theta \csc \beta, \\
\mathrm{a}_{\mathrm{c}} & =\mathrm{t} \sec \epsilon \csc \theta, \\
\mathrm{a} & =\mathrm{d}(\cos \theta+\sin \theta \cot \beta)-\mathrm{a}_{\mathrm{c}},
\end{aligned}
$$

and

$$
d V=\left(a+a_{c}\right)^{2} d \sin ^{2} \theta \csc ^{2} \beta d \beta d \theta d \epsilon .
$$

For a homogeneous air atmosphere, the application of this coordinate system to the exposure rate equation, Eq. (3-9), gives the final form (Ro82) of

$$
\begin{aligned}
& \dot{\mathrm{X}}=\frac{\mathrm{SKN}}{2 \pi \mathrm{d}} \int_{0}^{\beta_{\max }} \mathrm{d} \beta \frac{\mu_{\mathrm{en}}}{\rho} \sigma \hat{\mathrm{E}} \int_{\theta_{\min }}^{\theta_{\max }} \mathrm{d} \theta \hat{\mathrm{B}} \exp (-\mu \mathrm{a}-\hat{\mu} \mathrm{b}) \\
& \cdot \int_{0}^{\epsilon_{\max }} \mathrm{d} \epsilon \mathrm{B} \exp \left(-\mu_{\mathrm{c}} \mathrm{a}_{\mathrm{c}}\right) .
\end{aligned}
$$




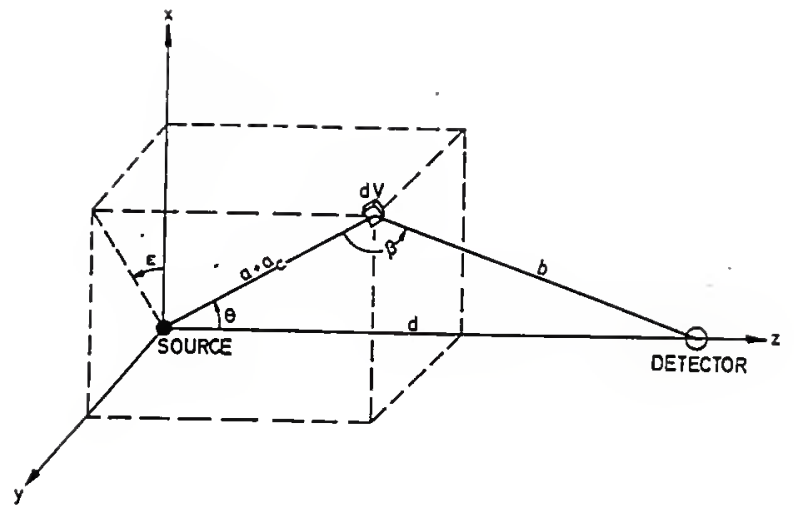

Fig. 3-6. Coordinate system for the single-scatter model as applied to the silo geometry. [From (Ros2)] 
Here, use has been made of symmetry in the variable $\epsilon$; since the last integrand is an even function of $\epsilon$, and $\epsilon_{\min }=-\epsilon_{\max }$, the integral from $\epsilon_{\min }$ to $\epsilon_{\max }$ may be replaced by twice the integral from 0 to $\epsilon_{\max }$. Also, it has been assumed that $a \gg a_{c}$, so that the distance $a$ is approximately independent of the angle $\epsilon$. For the case of an unshielded source, Eq. (3-14) reduces to (Ros2)

$$
\dot{\mathrm{X}}=\frac{\mathrm{SKN}}{2 \pi \mathrm{d}} \int_{0}^{\beta_{\max }} \mathrm{d} \beta \frac{\hat{\mu}_{\mathrm{en}}}{\rho} \sigma \dot{\mathrm{E}} \int_{0_{\min }}^{\theta_{\max }} \mathrm{d} \theta \hat{\mathrm{B}} \exp (-\mu \mathrm{a}-\hat{\mu} \mathrm{b}) \epsilon_{\max } \cdot(3-15)
$$

\subsubsection{Limits of Integration}

The values of the angles which make up the coordinate system are limited by the collimation of the silo and the restriction that the source, detector and scattering volume must form a closed triangle. The outer radius of the collimator silo is designated $\mathrm{r}$, as shown in Fig. 3-5. To describe the collimation, the variable $h$ is defined in Fig. $3-5(a)$ as the distance of the point source below the outer lip of the collimation silo, where no concrete roof is present. When a shield is present over the source, as in Fig. 3-5(b), the distance $\mathrm{h}$ cannot be easily defined, since the collimator is no longer atop the silo. Instead, the minimum value of $\theta$ may be defined to correspond to the longest gamma-ray path through the concrete shield that does not also pass through the silo, or the longest path through the shield that makes a significant contribution to the detector response. (Ro82) The distance h may then be computed as $\mathrm{I} \tan \theta_{\min }$. Note that $\mathrm{r}$ and $\mathrm{h}$ depend upon the outer edge of the silo; iu the MicroSkyshine code, for example, the inner dimensions 
of the silo determine $\mathrm{r}$ and $\mathrm{h}$, and care should be taken to avoid confusion of these definitions.

The limits of integration are related to the silo dimensions by the following formulas (Ro80, Ro82). Where the source is unshielded,

$$
\theta_{\min }=\arctan \frac{h}{r}
$$

If the silo is shielded, $\theta_{\min }$ is defined as mentioned above, with h computed from $\theta_{\min }$ and $\mathrm{r}$.

$$
\begin{aligned}
& \theta_{\max }=\min \left\{\left(\pi-\theta_{\min }\right), \pi-\beta-\arctan [\mathrm{h} /(\mathrm{d}-\mathrm{r})]\right\} \\
& \epsilon_{\max }=\arccos \left(\sin \theta_{\min } \csc \theta\right)
\end{aligned}
$$

In Roseberry's original code, the upper limit on $\beta$, the supplement of the scattering angle, depended on the silo height and the distance of the detector from the silo. Since the silo material is assumed to be a perfect absorber, photons were required to rise above the silo before scattering. This requirement took the following form:

$$
\beta_{\max }=\pi-\theta_{\min }-\arctan [\mathrm{h} /(\mathrm{d}-\mathrm{r})] .
$$

Note that $\beta_{\max }$ depends only on the problem geometry, not the variable $\theta$, since $\theta_{\min }$ is a constant for each geometry of source, detector and silo. The derivation of these limits is straightforward, and can be found in the thesis of Roseberry (Ros0). 
In reality, photons from an isotropic source in the silo may reflect off the interior walls of the silo, then scatter out the top and eventually contribute to the detector response. This was seen to some extent in benchmark experiments (Ros0). The use of Eq. (3-19) would not account for these photons and could result in an underestimate of detector response when compared to benchmark data. Faw and Shultis (Fa87) simulated the contributions of gamma rays which ricochet off the silo wall by allowing single scatters within the silo and computing detector responses from these "inside scatters," neglecting the shielding of the silo wall entirely. In a sense, MicroSkyshine uses the silo only to collimate the source photons into a known solid angle, not to restrict the space in which photons scatter. This conservative approach has been taken in modifying Roseberry's program for this work. Photons scattering within the silo and escaping are simulated by extending the region of integration into the silo, an area not visible from the detector. The limits on $\theta$ and $\epsilon$ remain the same, but the restriction of the silo wall is removed from Eq. (3-19), so that $\beta$ is limited only by the angle of collimation, that is, the possible directions of source photons. In this work,

$$
\beta_{\max }=\pi-\theta_{\min } .
$$

Photons which scatter within the silo follow a path directly to the detector; the "perfectly absorbing" silo material is ignored. This approach avoids possible underestimation of true exposures or dose. 


\subsubsection{Numerical Evaluation}

The silo skyshine problem was solved numerically by Roseberry (Ros0) using Eqs. (3-14) through (3-19). The computer code written for this purpose, SKY, employed triple trapezoidal integration to compute the exposure rate from an isotropic source emitting one gamma ray per second at a specified energy. The angle of collimation of the silo and the thickness of an overhead concrete shield, if present, could be specified. To normalize results, the areal density between source and detector (distance times air density) was computed and reported; exposure rates were multiplied by the square of the source-detector distance and divided by the solid angle of collimation of the source photons. These normalized exposure rates were displayed versus areal density for all comparisons.

Part of the work performed for this thesis involved modifications to SKY. Since the original code was written, improved cross sections and buildup factors have been published, and improvements have been made in numerical integration techniques. These data and methods have been incorporated into a new version of Roseberry's code, and comparisons have been made to investigate the effects of the changes. These comparisons and changes will be described in this section.

Numerical Approximations. The program written by Roseberry involved several approximations made to simplify coding of the exposure rate equation or to shorten run times (Ro80). Many of these were retained in subsequent versions of SKY, referred to in this work as SKYS and SILOGP. 
Although the angle $\beta$ has a theoretical lower limit of zero, this occurs when the scattering volume is infinitely far from the source and detector. Gamma rays are very unlikely to travel to such a point, and the contribution to the detector response will be negligible for cases where $\beta$ is small. Therefore, Roseberry placed a limit on the distance in air through which photons might travel; the distance over both legs of the journey was limited to $10 \mathrm{mfp}$ (mean free paths). This places a numerical lower limit on $\beta$ below which the integrand is approximately zero. For some later versions of SKY, this limit was increased to $20 \mathrm{mfp}$, although Roseberry showed that $10 \mathrm{mfp}$ was adequate for $\mathrm{d} \leq 700 \mathrm{~m}$. (Ros0) Following a similar analysis, Roseberry placed a limit of $15 \mathrm{mfp}$ on the photon path length in concrete. This yields a numerical upper limit on $\epsilon$, above which the integrand is negligible. In SKY 8 and SILOGP, this cutoff value was retained.

Equation (3-14) assumes that the distance a traveled by source photons is approximately independent of $\epsilon$. To carry through on the assumption, the concrete distance $a_{c}$ was dropped from Eq. (3-12) within the code SKY, leaving only $\theta$ and $\beta$ as the independent variables. For problems involving ${ }^{60} \mathrm{Co}$ point sources, Roseberry approximated the emission spectrum of 1.17 $\mathrm{MeV}$ and $1.33 \mathrm{MeV}$ photons by two $1.25 \mathrm{MeV}$ photons per disintegration. This customary approach was proven to change the computed exposure rates by less than two percent for all configurations studied. (Ro80) Both of these numerical approximations were also carried over into the revised versions of the code and problems performed with them.

The MicroSkyshine code (Fa87) allowed photons which first scatter within the silo to contribute to measured results. As discussed in the previous 
section, a similar approximation has been used in SKY8 and SHLOGP. However, approximations found in other works were not applied in modifying SKY. One of these, used in $\mathrm{G}^{3}$ (Ma73), was to "convert" the first-collision scattering source at $\mathrm{dV}$ to an equivalent isotropic source. This would justify the use of a buildup factor computed for a point isotropic source and solve the incongruity mentioned in section 3.1.2. However, the source term "conversion" performed by $\mathrm{G}^{3}$ is to simply multiply the photon flux density leaving the first-collision source in a unit steradian toward the detector by $4 \pi$. To find the flux density at the detector from this isotropic scattering source, the new source strength must be divided by $4 \pi b^{2}$. The $4 \pi$ terms cancel, and the end result is identical to Eq. $(3-6)$, where the scattering source term $S_{V}$ is directed into a unit steradian, and need only be divided by $b^{2}$ to account for inverse square attenuation. The conversion does not truly yield an equivalent isotropic scattering source, and is eliminated in the formulation, so it was not applied in this work.

Finally, the air density is taken to be constant over all regions in which gamma rays travel, and the atmosphere is taken to be homogeneous in composition. Since the distances involved in computations are not likely to exceed 5000 meters, this is a valid approximation; numerical estimates of attenuation through an altitude-dependent atmosphere, such as the method used by ZAP (Ma69), are avoided. For simplicity, the concrete shield placed over the source is also assumed to be homogencous. Approximating density changes in either air or concrete is rarely, if ever, required.

Use of Gauss Quadrature. The original code SKY written by Roseberry performed its numerical integration of Eq. (3-14) by triple trapezoidal 
integration. The three regions of integration (over $\beta, \theta$ and $\epsilon$ ) were each divided into ten regions, with a total of 1100 mesh points at which the integrand was evaluated. Roseberry found this to be an acceptable mesh size both in accuracy and in computation time. (Roso)

Gauss quadrature is commonly known to be more accurate than trapezoidal integration, and can achieve better results with fewer evaluations of the integrand than either trapezoidal integration or the use of Simpson's rule. Some recent computer codes use Gauss quadrature in some capacity, notably MORSE (We84) and MicroSkyshine (Fa87). Hornbeck (Ho75) comments that great accuracy is possible without many points, and that Gauss quadrature is "a very desirable method to use for multiple integration," since fewer evaluations of the integrands are necessary than with other methods, and less error accumulates in the outer integral as a result. Details of the technique may be found in any of the three references above.

In the first modification to SKY, named SKY8, the trapezoidal integration formulas were replaced by function subprograms and a Gauss quadrature subroutine. The subroutine used here originated at Sandia National Laboratory, and performs adaptive quadrature; that is, separate integrations are performed over successively smaller sections of the entire region of integration until the change in the result is below a chosen criterion. SKY and SKYS were applied to two different silo geometries, one with an unshielded, collimated silo, the other with a concrete shield $21 \mathrm{~cm}$ thick over the silo. The source in both cases was a ${ }^{60} \mathrm{Co}$ point source, normalized to a strength of one photon per second. As shown in Tables 3-1 and 3-2, the change in exposure rates is minimal, less than $2 \%$ in all cases. Because the Gaussian 
Table 3-1. Comparison of methods of numerical integration as applied to the computer code SkY. The exposures are for a ${ }^{60} \mathrm{Co}$ point source on the axis of an open silo with a solid angle of collimation of $4.683 \mathrm{sr}$. Air density is taken as $1.2 \mathrm{mg} / \mathrm{cm}^{3}$.

\begin{tabular}{|c|c|c|c|}
\hline \multirow[b]{2}{*}{$\begin{array}{l}\text { Source-detector } \\
\text { distance (m) }\end{array}$} & \multirow[b]{2}{*}{$\begin{array}{l}\text { Areal density } \\
\left(\mathrm{g} / \mathrm{cm}^{2}\right)\end{array}$} & \multicolumn{2}{|c|}{$\begin{array}{c}\text { Normalized exposure } \\
{\left[\mathrm{m}^{2} \cdot \mathrm{R} /(\mathrm{sr} \cdot \text { photon })\right]}\end{array}$} \\
\hline & & $\begin{array}{l}\text { Computed by } \\
8 \text {-point } \\
\text { Gauss } \\
\text { quadrature }\end{array}$ & $\begin{array}{l}\text { Computed by } \\
\text { 11-point } \\
\text { trapezoidal } \\
\text { integration }\end{array}$ \\
\hline $\begin{array}{r}25.0 \\
37.5 \\
50.0 \\
62.5 \\
75.0 \\
87.5 \\
100.0 \\
112.5 \\
137.5 \\
150.0 \\
162.5 \\
175 \\
200 \\
225 \\
250 \\
275 \\
300 \\
350 \\
400 \\
450 \\
500 \\
550 \\
600 \\
650 \\
700 \\
750 \\
800 \\
850 \\
900 \\
950\end{array}$ & $\begin{array}{r}3.0 \\
4.5 \\
6.0 \\
7.5 \\
9.0 \\
10.5 \\
12.0 \\
13.5 \\
16.5 \\
18.0 \\
19.5 \\
21 \\
24 \\
27 \\
30 \\
33 \\
36 \\
42 \\
48 \\
54 \\
60 \\
66 \\
72 \\
78 \\
84 \\
90 \\
96 \\
102 \\
108 \\
114\end{array}$ & $\begin{array}{l}2.853(-17) * \\
4.182(-17) \\
5.358(-17) \\
6.367(-17) \\
7.209(-17) \\
7.892(-17) \\
8.429(-17) \\
8.831(-17) \\
9.292(-17) \\
9.377(-17) \\
9.384(-17) \\
9.323(-17) \\
9.038(-17) \\
8.598(-17) \\
8.058(-17) \\
7.462(-17) \\
6.842(-17) \\
5.621(-17) \\
4.509(-17) \\
3.554(-17) \\
2.764(-17) \\
2.126(-17) \\
1.621(-17) \\
1.227(-17) \\
9.225(-18) \\
6.902(-18) \\
5.142(-18) \\
3.816(-18) \\
2.821(-18) \\
2.080-18)\end{array}$ & $\begin{array}{l}2.832(-17) \\
4.152(-17) \\
5.319 \\
6.321 \\
\begin{array}{l}-17 \\
7.157\end{array}(-17) \\
7.836(-17) \\
8.369(-17) \\
8.768(-17) \\
9.226(-17) \\
9.312(-17) \\
9.318(-17) \\
9.258(-17) \\
8.976(-17) \\
8.538(-17) \\
8.002(-17) \\
7.409(-17) \\
6.793(-17) \\
5.579(-17) \\
4.475(-17) \\
3.526(-17) \\
2.740(-17) \\
2.106(-17) \\
1.604(-17) \\
1.213(-17 \\
9.115(-18) \\
6.814(-18) \\
5.071(-18) \\
3.759(-18\} \\
2.777(-18) \\
2.045(-18)\end{array}$ \\
\hline
\end{tabular}


Table 3-2. Comparison of methods of numerical integration as applied to the computer code SKY. The exposures are for a $60^{\circ} \mathrm{Co}$ point source on the axis of a silo, shielded by a concrete slab of density $2.13 \mathrm{~g} / \mathrm{cm}^{3}$ and thickness $21 \mathrm{~cm}$. Air density is taken as $1.2 \mathrm{mg} / \mathrm{cm}^{3}$.

\begin{tabular}{|c|c|c|c|}
\hline \multirow[b]{2}{*}{$\begin{array}{l}\text { Source-detector } \\
\text { distance (m) }\end{array}$} & \multirow[b]{2}{*}{$\begin{array}{l}\text { Areal density } \\
\left(\mathrm{g} / \mathrm{cm}^{2}\right)\end{array}$} & \multicolumn{2}{|c|}{$\begin{array}{l}\text { Normalized exposure } \\
{\left[\mathrm{m}^{2} \cdot \mathrm{R} /(\mathrm{sr} \cdot \text { photon })\right]}\end{array}$} \\
\hline & & $\begin{array}{l}\text { Computed by } \\
\text { 8-point } \\
\text { Gauss } \\
\text { quadrature }\end{array}$ & $\begin{array}{l}\text { Computed by } \\
\text { 11-point } \\
\text { trapezoidal } \\
\text { integration } \\
\end{array}$ \\
\hline $\begin{array}{r}25.0 \\
37.5 \\
50.0 \\
62.5 \\
75.0 \\
87.5 \\
100.0 \\
112.5 \\
137.5 \\
150.0 \\
162.5 \\
175 \\
200 \\
225 \\
250 \\
275 \\
300 \\
350 \\
400 \\
450 \\
500 \\
550 \\
600 \\
650 \\
700 \\
750 \\
800 \\
850 \\
900 \\
950\end{array}$ & $\begin{array}{r}3.0 \\
4.5 \\
6.0 \\
7.5 \\
9.0 \\
10.5 \\
12.0 \\
13.5 \\
16.5 \\
18.0 \\
19.5 \\
21 \\
24 \\
27 \\
30 \\
33 \\
36 \\
42 \\
48 \\
54 \\
60 \\
66 \\
72 \\
78 \\
84 \\
90 \\
96 \\
102 \\
108 \\
114\end{array}$ & $\begin{array}{l}2.412(-18))^{*} \\
3.516(-18) \\
4.460(-18) \\
5.236(-18) \\
5.847-18) \\
6.308(-18) \\
6.634(-18) \\
6.841(-18) \\
6.968(-18) \\
6.917(-18) \\
6.808(-18) \\
6.652 \\
6.237-18 \\
5.738(-18) \\
5.200(-18) \\
4.658(-18) \\
4.133(-18) \\
3.182(-18) \\
2.395(-18) \\
1.773(-18) \\
1.298(-18) \\
9.408(-19) \\
6.772(-19) \\
4.848(-19) \\
3.454(-19) \\
2.452(-19) \\
1.737(-19) \\
1.227(-19) \\
8.650(-20) \\
6.087(-20)\end{array}$ & 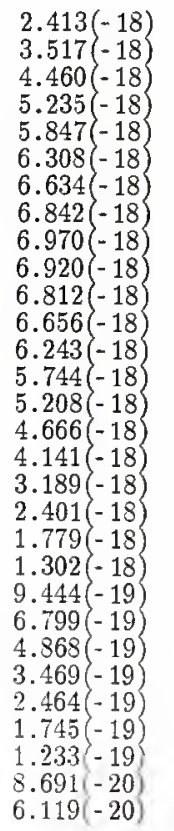 \\
\hline
\end{tabular}

$* 2.412(-18)=2.412 \times 10^{-18}$ 
quadrature method is known to be more accurate, it has been retained in the code.

Data Used in Codes. The single-scatter and buildup method requires two data bases: one of gamma-ray interaction coefficients, the other of parameters used in computing buildup factors. In SKY, Roseberry employed attenuation coefficients published by the National Bureau of Standards; the data are over twenty years old, however, and more recent data can be obtained from several sources. Buildup was computed in SKY by the Berger formula, with published coefficients used as the data base. A new formula for photon buildup known as geometric progression has become popular for numerical use; although the formula is complex compared to the Berger approximation, the results agree more closely with experimental and computational data (Ha83, Ha86). It was decided to bring these two portions of the code up to date and compare results to determine what changes, if any, would result in the exposure rates from the modifications.

The first alteration made to SKY 8 was the replacement of the Berger buildup formula by the geometric progression formula of Harima et al. (Has6). An early version of the formula (Has3) was presented as an alternative to other gamma-ray buildup approximations, which were fit to reported data but did not reproduce them well. Some buildup formulas deviated as much as $40 \%$ from widely-used moments method data; one approximation was cited as giving negative buildup factors when extrapolated. Harima obtained coefficients for the first version of the geometric progression from fits to numerical moments-method results and results of other computer codes. 
Except for lead, the geometric-progression (or GP) buildup factors were well within $10 \%$ of the original data. The coefficients were easily interpolated, another advantage of the GP method.

The current version of the GP buildup formula (Ha86) was published by Harima et al. three years later. The authors explain the physical meaning of each term in this more accurate form, report coefficients for several materials over different energy ranges, suggest an extrapolation method for large distances, and recommend parabolic interpolation in energy and distance. The formula and interpolation scheme were used by Shultis and Faw (Sh87) in computing response functions for the MicroSkyshine code, and are employed by Cain and Trubey (RS86) in a recent version of the QAD program for microcomputers. It was this revised formula that was included in the modified version of SKY8, renamed SILOGP.

The GP approximating function for the gamma-ray point-source buildup factor is defined as (Ha86, RSs6)

$$
\mathrm{B}\left(\mathrm{E}_{0}, \mathrm{x}\right)=\left\{\begin{array}{ll}
1+(b-1) \frac{\mathrm{K}^{\mathrm{x}}-1}{\mathrm{~K}-1} & , \quad \mathrm{~K} \neq 1 \\
1+(b-1) \mathrm{x} & , \quad \mathrm{K}=1
\end{array}\right\}
$$

where

$$
\mathrm{K}=\mathrm{cx}^{\mathrm{a}}+\mathrm{d} \frac{\tanh \left(\mathrm{x} / \mathrm{X}_{\mathrm{k}}-2\right)-\tanh (-2)}{1-\tanh (-2)}
$$

and

$$
\begin{aligned}
\mathrm{E}_{0}= & \text { gamma-ray source energy } \\
\mathrm{x}= & \text { source-detector distance in the medium in mean free } \\
& \text { paths, }
\end{aligned}
$$




$$
\mathrm{b}, \mathrm{c}, \mathrm{a}, \mathrm{d}, \mathrm{X}_{\mathrm{k}}=\underset{\text { parameters dependent on } \mathrm{E}_{0} \text { and the material of the }}{\text { medium. }}
$$

The values for the parameters used in this work are those used in QAD-CGGP (RS86); the subroutines used to calculate the buildup factors are identical to those in QAD, with a modification to correct an error in the parabolic interpolation procedure.

For comparison of buildup, the unshielded and shielded silo geometries used in testing the Gauss quadrature subroutines were again used. Normalized exposure rates were computed along a radial from the source out to $950 \mathrm{~m}$ using the Berger buildup formula, and again with the GP buildup formula. The results are shown in Fig. 3-7. Differences in the buildup are immediately seen; for tbe unsbielded case, wbere buildup is only used in air, the normalized exposure profile computed with GP factors shows less variation over distance than the Berger-formula exposure profile. The tendency of the Berger factors to overestimate close to the source is especially evident here. The GP buildup method was chosen for all subsequent work.

The other change in the data base was an update of interaction coefficients. Tbe twenty-year-old NBS values used by Roseberry were replaced by those published in an article by Hubbell (Hu82). This compilation, also performed for the National Bureau of Standards, contains values of $\mu / \rho$ and $\mu_{\mathrm{en}} / \rho$ for forty elements and forty-five mixtures over the range of $1 \mathrm{keV}$ to 20 $\mathrm{MeV}$. Very little of the data is empirical; the mass attenuation and mass energy absorption coefficients were obtained from tbeory, with empirical adjustments made to $\mu_{\mathrm{en}} / \rho$. Values for mixtures were computed from the elemental data and appropriate weight fractions. Of special note is the White-Grodstein composition for concrete used by NBS, which differs from the 


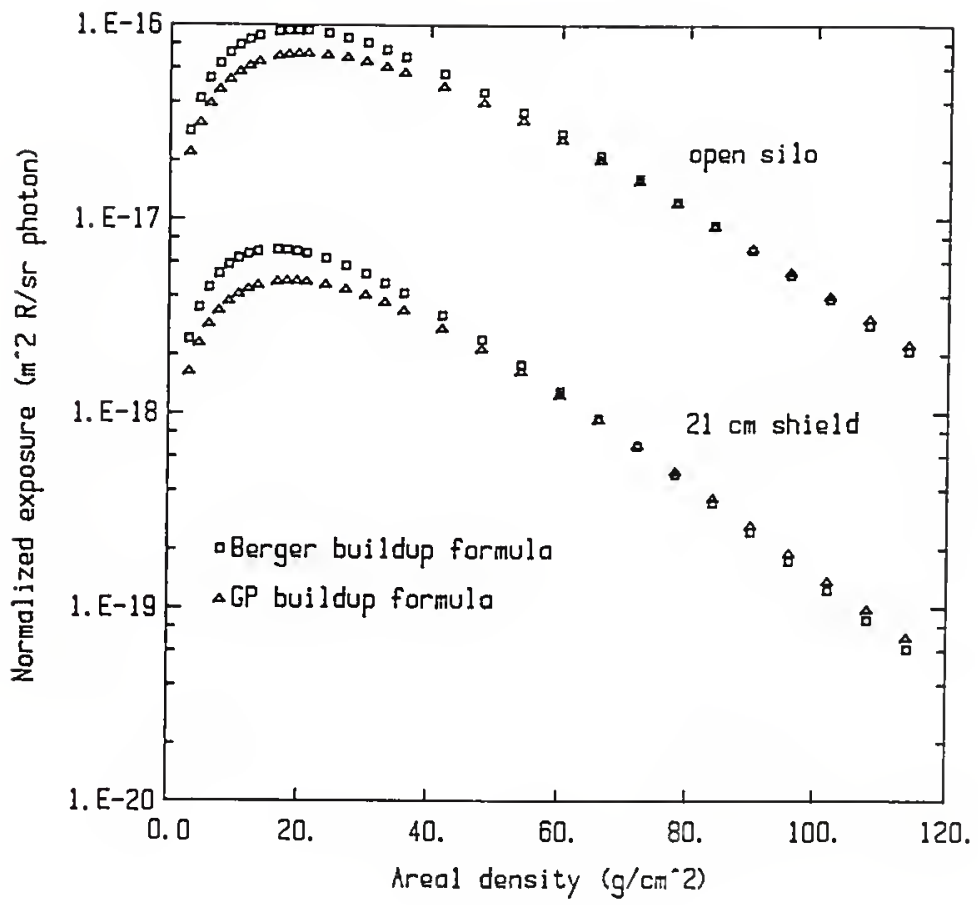

Fig. 3-7. Comparison of exposures computed by the computer code SKY8 using the Berger and geometric progression (GP) buildup formulas. The exposures are for a ${ }^{60} \mathrm{Co}$ point source on the axis of a silo, with and without overhead concrete shielding. 
composition used for ANSI-standard problems (AN87). This difference was neglected for later comparisons to ANSI reference results.

Exposure rates were computed with the old and new interaction coefficients to compare clianges in detector response. The same silo problems were used in this test as were used with the integration and buildup factor comparisons. Also, since the decision to use the new data was made well after the GP buildup formula was incorporated, both sets of computations employed adaptive Gaussian 8-point quadrature and GP buildup factors. The results are presented in Tables 3-3 and 3-4. For the case of an unshielded, collimated source, the change in the exposure rate due to the change in $\mu / \rho$ values is less than $2 \%$, even at large distances. This is to be expected, since Hubbell (Hu82) reported that differences in the data from earlier standard values are only $5 \%$ in the worst cases. For the shielded silo case, the data from Hubbell produce exposures which are consistently lower, but the change is less than $3 \%$, even as the source-detector distance approaches 1000 meters.

The final version of the silo skyshine code SILOGP, including Gauss quadrature, the geometric-progression buildup formula, and new interaction coefficients, is listed in Appendix A. In Chapter 4 this version will be compared to other methods and validated against reference data.

\subsection{Application of the Exposure Rate Integral Equation to a Wall Geometry} The other problem addressed in this thesis is that of a point source of gamma rays separated from a point detector by a semi-infinite wall. The wall geometry is shown in simple form in Fig. 3-8, with distances between the source, detector, and scattering volume labeled; the Cartesian dimensions which 
Table 3-3. Comparison of mass interaction data used in the computer code SILOGP. The exposures are for a $60 \mathrm{Co}$ point source on the axis of an open silo with a solid angle of collimation of $4.683 \mathrm{sr}$. Air density is taken as $1.2 \mathrm{mg} / \mathrm{cm}^{3}$.

\begin{tabular}{|c|c|c|c|}
\hline \multirow[b]{2}{*}{$\begin{array}{l}\text { Source- detector } \\
\text { distance (m) }\end{array}$} & \multirow[b]{2}{*}{$\begin{array}{l}\text { Areal density } \\
\left(\mathrm{g} / \mathrm{cm}^{2}\right)\end{array}$} & \multicolumn{2}{|c|}{$\begin{array}{l}\text { Normalized exposure } \\
{\left[\mathrm{m}^{2} \cdot \mathrm{R} /(\mathrm{sr} \cdot \text { photon })\right]}\end{array}$} \\
\hline & & $\begin{array}{l}\mu / \rho \text { from } \\
\text { Roseberry } \\
(\text { Ro } 80)\end{array}$ & $\begin{array}{l}\mu / \rho \text { from } \\
\text { Hubbel1 } \\
(\mathrm{Hu} 82)\end{array}$ \\
\hline $\begin{array}{l}25.0 \\
37.5 \\
50.0 \\
62.5 \\
75.0 \\
87.5 \\
100.0 \\
112.5 \\
137.5 \\
150.0 \\
162.5 \\
175 \\
200 \\
225 \\
250 \\
275 \\
300 \\
350 \\
400 \\
450 \\
500 \\
550 \\
600 \\
650 \\
700 \\
750 \\
800 \\
850 \\
900 \\
950\end{array}$ & $\begin{array}{r}3.0 \\
4.5 \\
6.0 \\
7.5 \\
9.0 \\
10.5 \\
12.0 \\
13.5 \\
16.5 \\
18.0 \\
19.5 \\
21 \\
24 \\
27 \\
30 \\
33 \\
36 \\
42 \\
48 \\
54 \\
60 \\
66 \\
72 \\
78 \\
84 \\
90 \\
96 \\
102 \\
108 \\
114\end{array}$ & 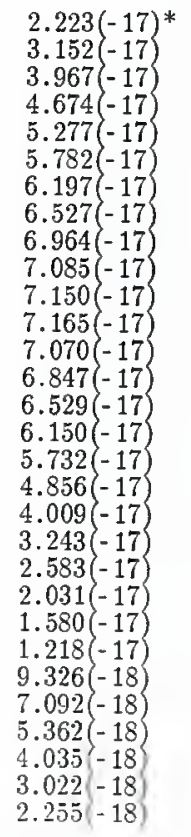 & 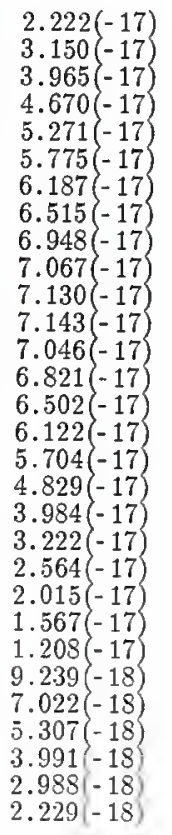 \\
\hline
\end{tabular}


Table 3-4. Comparison of mass interaction data used in the computer code SILOGP. The exposures are for a $60 \mathrm{Co}$ point source on the axis of a silo, shielded by a concrete slab of density $2.13 \mathrm{~g} / \mathrm{cm}^{3}$ and thickness 21 $\mathrm{cm}$. Air density is taken as $1.2 \mathrm{mg} / \mathrm{cm}^{3}$.

\begin{tabular}{|c|c|c|c|}
\hline \multirow[b]{2}{*}{$\begin{array}{l}\text { Source- detector } \\
\text { distance (m) }\end{array}$} & \multirow[b]{2}{*}{$\begin{array}{l}\text { Areal density } \\
\left(\mathrm{g} / \mathrm{cm}^{2}\right)\end{array}$} & \multicolumn{2}{|c|}{$\begin{array}{l}\text { Normalized exposure } \\
{\left[\mathrm{m}^{2} \cdot \mathrm{R} /(\mathrm{sr} \cdot \text { photon })\right]}\end{array}$} \\
\hline & & $\begin{array}{l}\mu / \rho \text { from } \\
\text { Roseberry } \\
\text { (Ros0) }\end{array}$ & $\begin{array}{l}\mu / \rho \text { from } \\
\text { Hubbe11 } \\
\text { (Hu82) }\end{array}$ \\
\hline $\begin{array}{l}25.0 \\
37.5 \\
50.0 \\
62.5 \\
75.0 \\
87.5 \\
100.0 \\
112.5 \\
137.5 \\
150.0 \\
162.5 \\
175 \\
200 \\
225 \\
250 \\
275 \\
300 \\
350 \\
400 \\
450 \\
500 \\
550 \\
600 \\
650 \\
700 \\
750 \\
800 \\
850 \\
900 \\
950\end{array}$ & $\begin{array}{r}3.0 \\
4.5 \\
6.0 \\
7.5 \\
9.0 \\
10.5 \\
12.0 \\
13.5 \\
16.5 \\
18.0 \\
19.5 \\
21 \\
24 \\
27 \\
30 \\
33 \\
36 \\
42 \\
48 \\
54 \\
60 \\
66 \\
72 \\
78 \\
84 \\
90 \\
96 \\
102 \\
108 \\
114\end{array}$ & 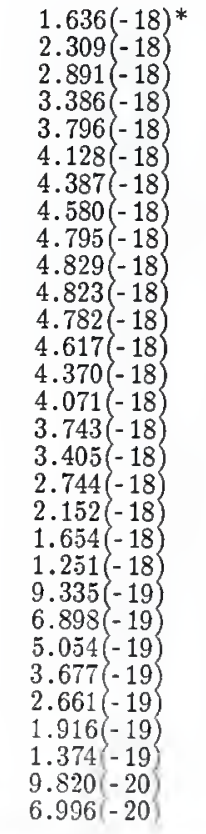 & 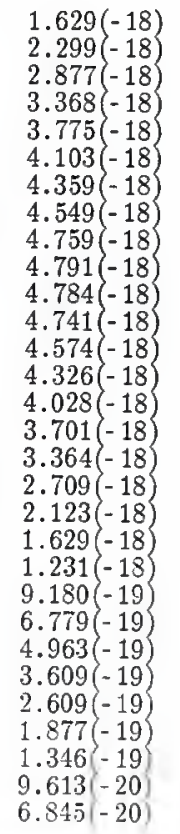 \\
\hline
\end{tabular}

* $1.636(-18)=1.636 \times 10^{-18}$ 

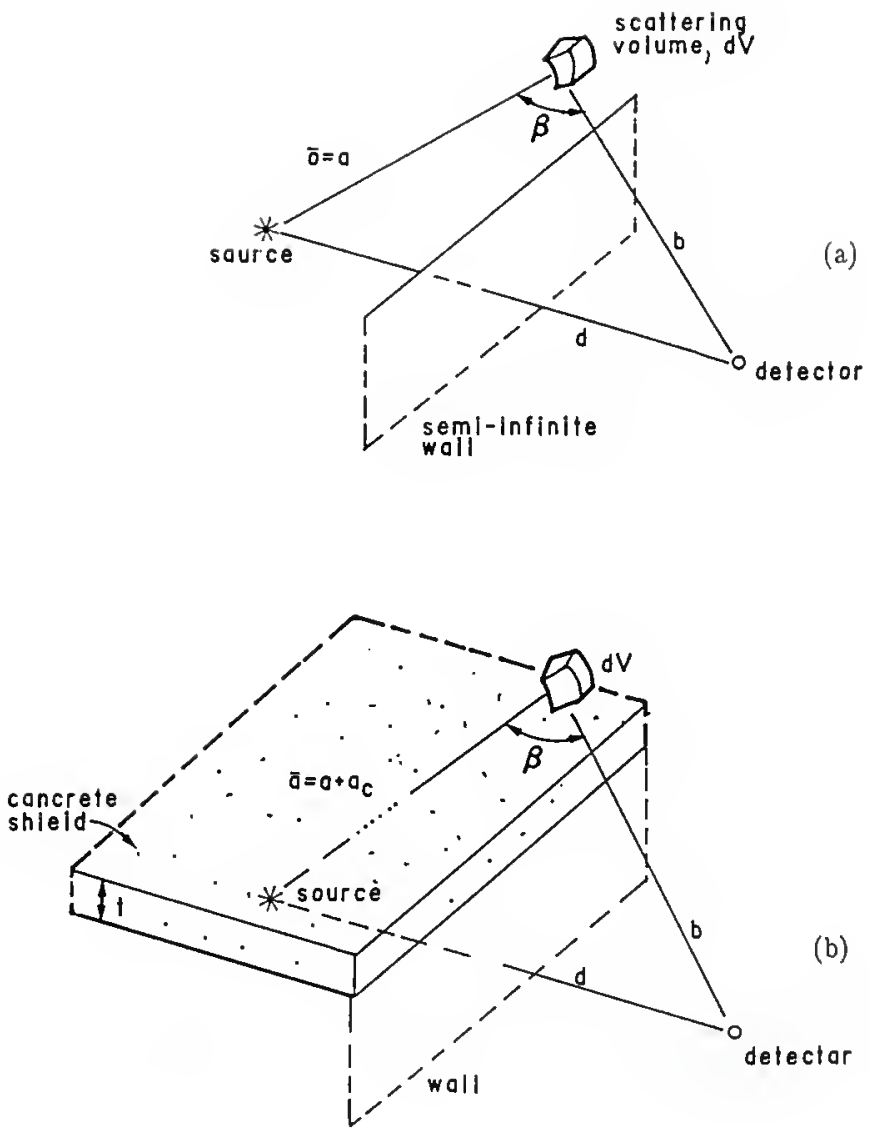

Fig. 3-8. Simplified calculational geometry for the case of a point source behind a semi-infinite wall: (a) no overhead shield; (b) overhead concrete roof present. 
locate the source, detector and wall in space are presented in Figs. 3-9 and 3-10. The wall extends downward and sideways to infinity and is of perfect absorbing material. The point source lies a distance $x_{5}$ from the wall, measured perpendicular to the wall, and lies a distance $y_{s}$ below the top edge of the wall. The point detector lies a distance $x_{d}$ from the wall face and $y_{d}$ below the wall edge. To measure the "offset" of the source and detector, a vertical plane is extended from the source through the wall, normal to the wall. The distance from the detector to this plane, or the "offset," is $z_{\mathrm{d}}$. If either the source or detector (never both) is above the wall edge, $y_{s}$ or $y_{d}$ is negative, respectively. The value of $z_{d}$ is taken as positive in the direction shown in Fig. 3-9.

The source and detector may or may not lie on a line normal to the wall, and may have different heights with respect to the top of the wall, but must be separated along the line of sight by the wall. This restriction forces photons to scatter in air to reach the detector. As in the silo problem, the source may be open to the air or covered by a concrete shield of thickness $t$. The source-detector distance is again designated as $d$. No ground-air interface is involved in this problem, for simplicity, and the thickness of the wall is negligible.

To the author's knowledge, the wall problem lias only been addressed previously by Faw and Shultis (Fas7) using MicroSkyshine. Their coordinate system differs from the one developed for this work, due to the requirements of MicroSkyshine. Results of the two methods will be compared in the next chapter. 


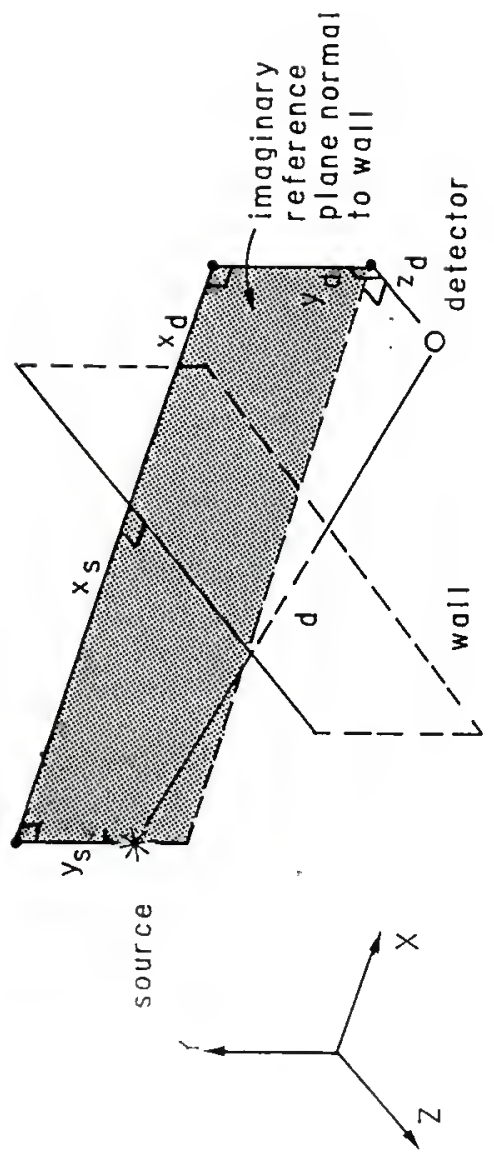

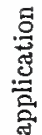

号

晜

ซี

율윰

$\infty=$

콜

농

월워

亲

吾

D.

क

年 

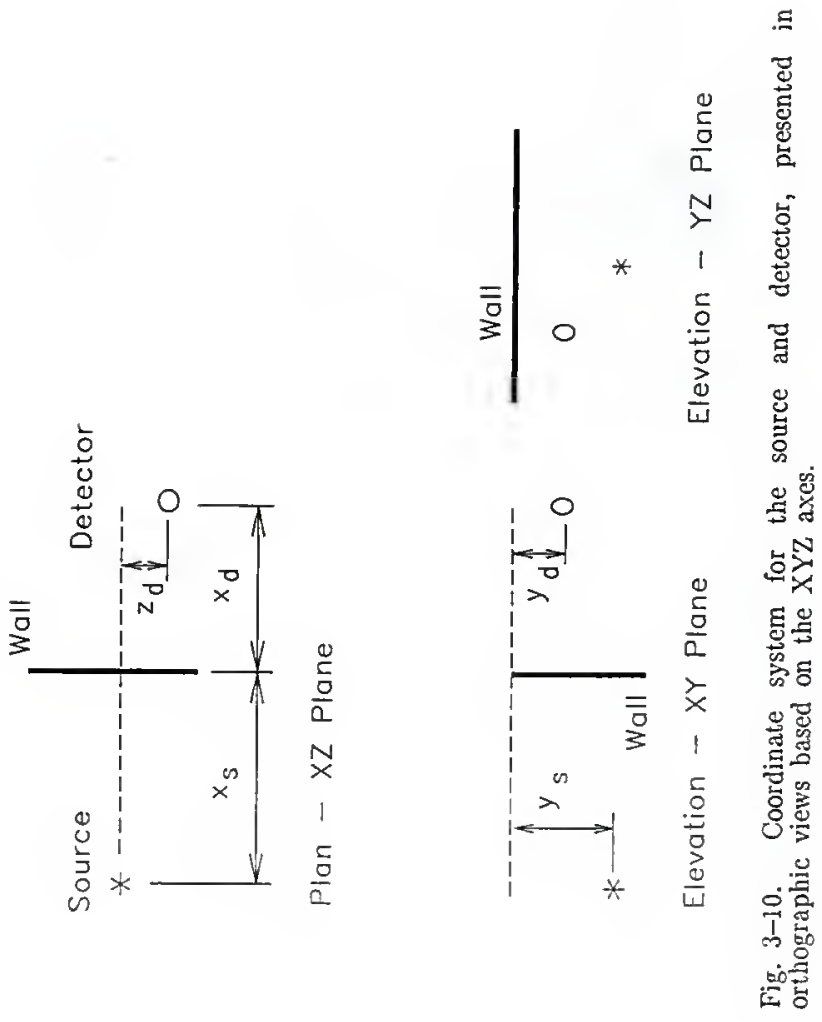


\subsubsection{Coordinate Systems}

The exposure rate equation which is to be solved for the wall case is repeated here.

$$
\dot{\mathrm{X}}=\int_{\mathrm{V}} \frac{\mathrm{SKNBB} \sigma}{4 \pi\left(\mathrm{a}+\mathrm{a}_{\mathrm{c}}\right)^{2} \mathrm{~b}^{2}} \frac{\dot{\mu}_{\mathrm{en}}}{\rho} \hat{\mathrm{E}} \exp \left(-\mu \mathrm{a}-\mu_{\mathrm{c}} \mathrm{a}_{\mathrm{c}}-\hat{\mu} \mathrm{b}\right) \mathrm{dV}
$$

To integrate Eq. (3-9) over all space in which a gamma ray may scatter once and reach the detector directly, a three-dimensional coordinate system must again be selected. The system of angles used in the silo problem by Roseberry was chosen first, but produced an extremely large and cumbersome set of limits, many of which were valid only for certain positions of source and detector. It was discovered that a simple cylindrical coordinate system could be applied instead, requiring fewer equations to determine the limits of integration. Thus the cylindrical system was used in the numerical analysis.

Using the cylindrical coordinate system requires that the source, detector and wall positions be translated to cylindrical coordinates. By definition, the source lies at the origin and the point detector is at $\mathrm{r}=0, \mathrm{z}=\mathrm{d}$. Only the edge of the wall is of concern, and it may be described by an infinite line. The translation begins with the rotation of the Cartesian geometry of Fig. 3-10 into a second Cartesian geometry, in which the source and detector lie the same distance $\mathrm{h}$ below the wall. The rotation is presented in Fig. 3-11, and its result is slown in Fig. 3-12. The intermediate angles and quantities are as follows:

$$
\theta=\arctan \frac{y_{s}-y_{d}}{x_{s}+x_{d}}
$$




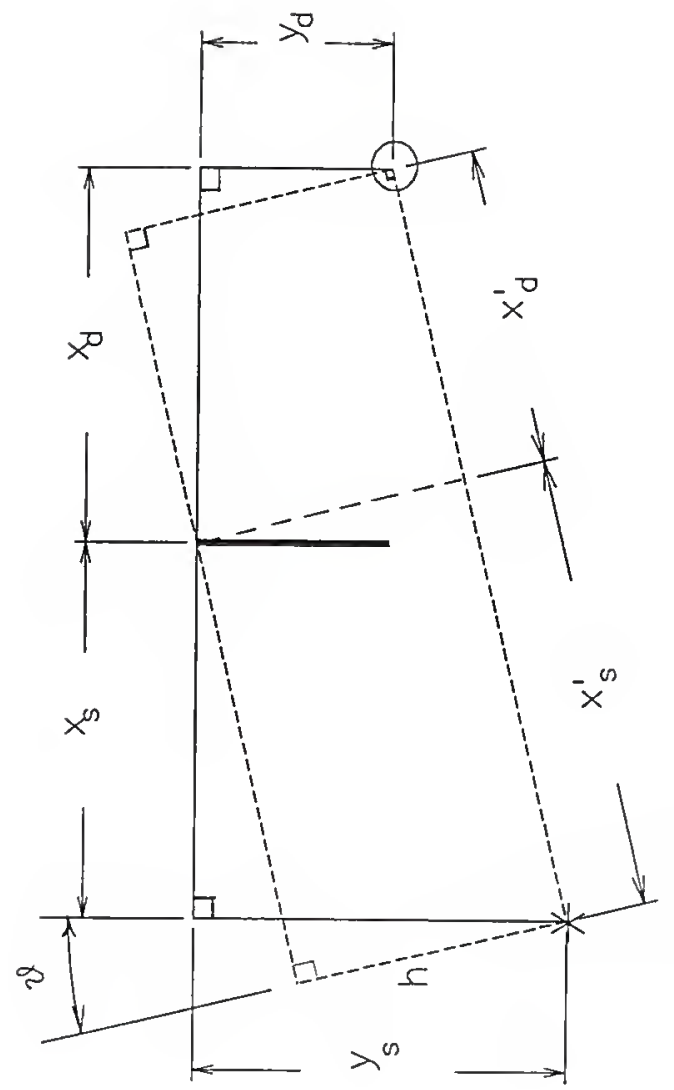

1)

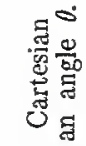

동

을

莺岂

홍

sos

콬

峰愛

宁

密

U E

夈 

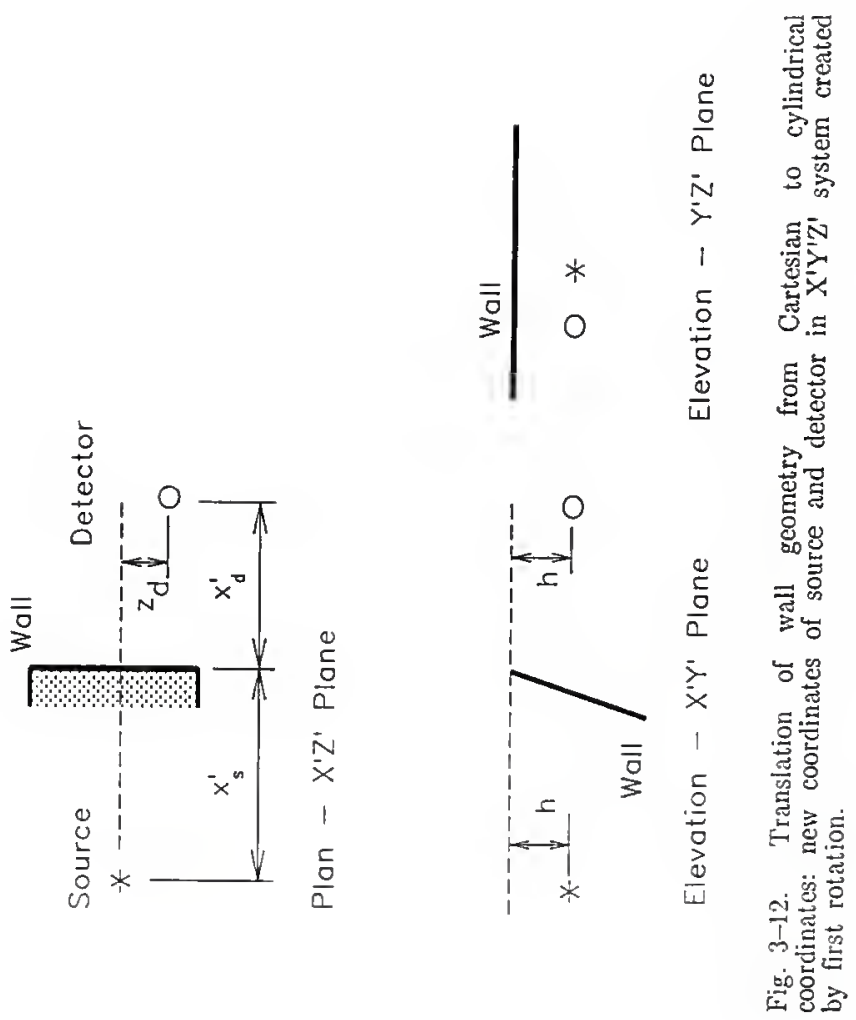


$$
\begin{aligned}
\mathrm{h} & =\mathrm{y}_{\mathrm{s}} \cos \theta-\mathrm{x}_{\mathrm{s}} \sin \theta=\mathrm{y}_{\mathrm{d}} \cos \theta+\mathrm{x}_{\mathrm{d}} \sin \theta \\
\mathrm{x}_{\mathrm{s}}^{\prime} & =\mathrm{x}_{\mathrm{s}} \cos \theta+\mathrm{y}_{\mathrm{s}} \sin \theta \\
\mathrm{x}_{\mathrm{d}}^{\prime} & =\mathrm{x}_{\mathrm{d}} \cos \theta-\mathrm{y}_{\mathrm{d}} \sin \theta
\end{aligned}
$$

The $X$ and $Y$ axes are rotated through an angle $\theta$ to become the $X^{\prime}$ and $Y^{\prime}$ axes. Note that the $Y^{\prime}$ axis lies in the $X Y$ plane, which acts as a reference for the offset $z_{d}$, and that $Y^{\prime}$ is perpendicular to the source-detector axis, which lies in the $X^{\prime} Z^{\prime}$ plane.

The second step of the transformation is another rotation of Cartesian axes. The $X^{\prime}$ and $Z$ axes are rotated through an angle $\xi$ to become the $X^{\prime \prime}$ and $Z^{\prime \prime}$ axes, as shown in Fig. 3-13. The axis $Y^{\prime}$ remains unchanged, and is identical to the $\mathrm{Y}^{\prime \prime}$ axis. The angle $\xi$ is defined such that the source detector axis is parallel to the $\mathrm{X}^{\prime \prime}$ axis, and is given by

$$
\xi=\arctan \frac{z}{x_{s}^{1}+x_{d}^{r}}
$$

The result of this second rotation is shown in Fig. 3-14. The new quantities are defined by

$$
\begin{aligned}
& x_{s}^{\prime \prime}=x_{s}^{\prime} \sec \xi, \\
& x_{d}^{\prime \prime}=x_{d}^{\prime} \sec \xi .
\end{aligned}
$$

Note that $x_{\mathrm{s}}^{\prime \prime}+\mathrm{x}_{\mathrm{d}}^{\prime \prime}=\mathrm{d}$. Here, $x_{\mathrm{s}}^{\prime \prime}$ represents the distance along the source-detector axis from the source to the top of the wall, while $x_{d}^{\prime \prime}$ is the 


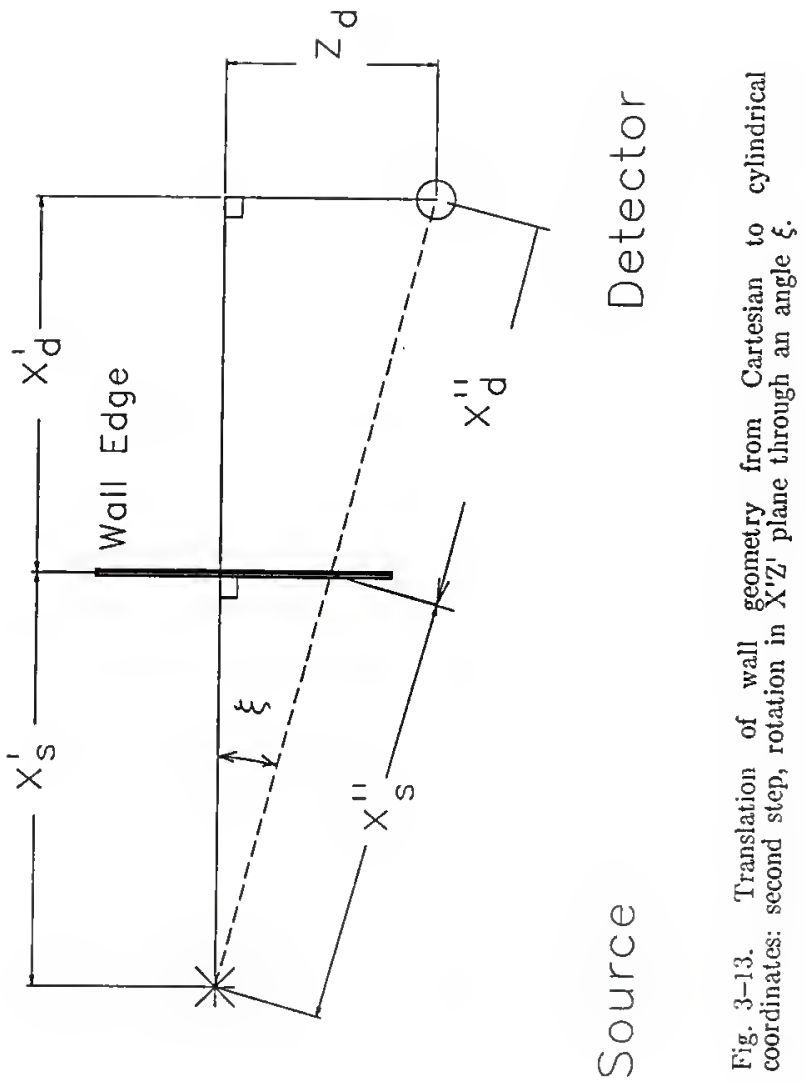



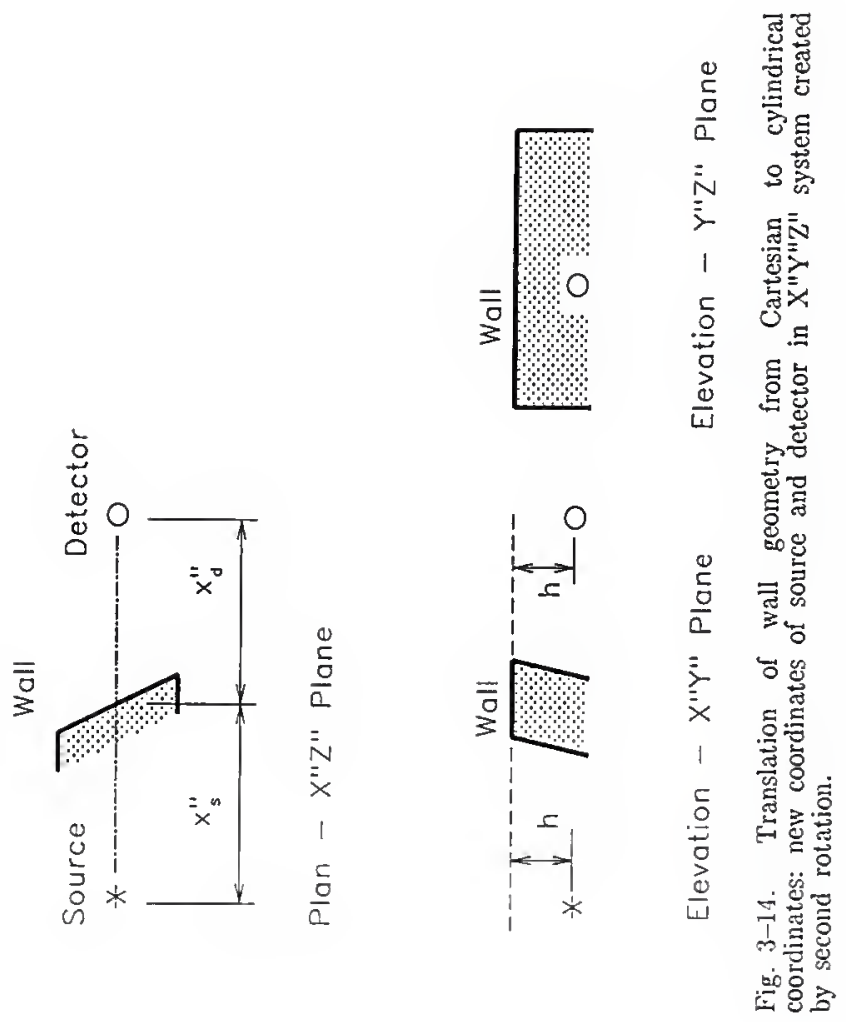
distance from the detector to the top of the wall. These two quantities directly affect limits of integration, as will be shown later.

Finally, the cylindrical coordinate system $(r, \phi, z)$ is superimposed on the third Cartesian system (X", Y", Z") in Fig. 3-15. The cylindrical polar axis is taken as the source-detector axis, with the point source located at the origin of the system and the detector at $\mathrm{z}=\mathrm{d}$. The radial coordinate $\mathrm{r}$ of the scattering volume $d V$ is measured from this axis. The polar axis is parallel to the $X^{\prime \prime}$ axis, hence the polar coordinate $\mathrm{z}$ is taken as positive in the positive $x^{\prime \prime}$ direction. The quantities $I$ and $\phi$ are defined in a plane parallel to the $Y^{\prime \prime} Z^{\prime \prime}$ plane, with the positive $Y^{\prime \prime}$ axis acting as the $\phi=0$ direction. The angle $\phi$ is measured using the right-hand rule about the $X^{\prime \prime}$ axis. Since the $Y^{\prime \prime}$ and $Y^{\prime \prime}$ axes are equivalent, $Y^{\prime}$ is normal to the source-detector axis, and the $X^{\prime} Y^{\prime}$ plane acts as a reference for the offset $z_{d}$, it is concluded that the reference plane for $\phi$ must include the source-detector axis, and must intersect the $z_{d}$ reference plane to form a line perpendicular to that axis.

The complement $\beta$ of the scattering angle is still required, since the Compton scattering parameters depend upon it. Given the cylindrical coordinates $(r, \phi, z)$ of the scattering volume, $\beta$ may he found. From Figs. 3-\$ and $3-15$,

$$
\beta=\operatorname{arccot} \frac{r}{z}+\operatorname{arccot} \frac{r}{d-z} .
$$

Other quantities can be obtained from the Pythagorean theorem: 


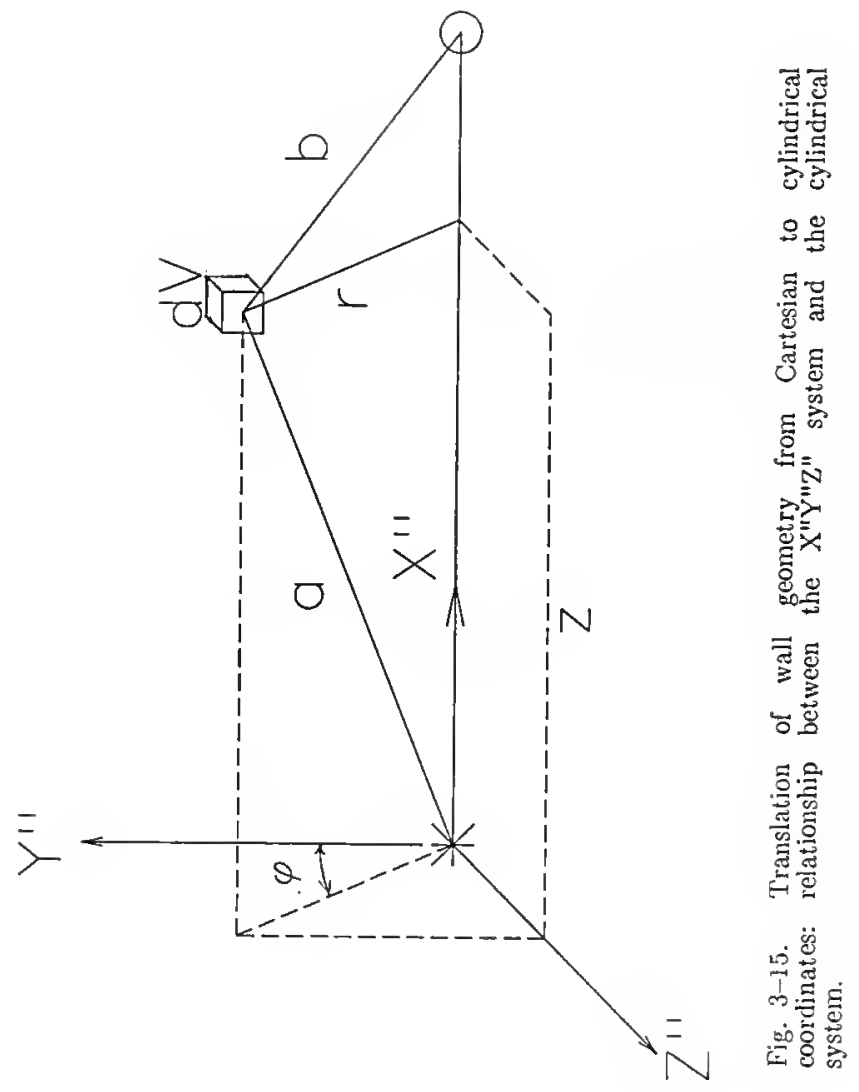




$$
\begin{aligned}
& \mathrm{b}=\sqrt{(\mathrm{d}-\mathrm{z})^{2}+\mathrm{r}^{2}}, \\
& \mathrm{a}=\sqrt{\mathrm{z}^{2}+\mathrm{r}^{2}}-\mathrm{ac}_{c} .
\end{aligned}
$$

The element of volume is

$$
\mathrm{dV}=\mathrm{r} \mathrm{dr} d \phi \mathrm{dz}
$$

The development of an accurate formula for the path length $a_{c}$ in concrete is complicated, because the concrete slab may be skewed with respect to the source-detector axis and the cylindrical coordinate system. Figure 3-16 illustrates the components of the path length $a_{c}$, taken as XYZ components because the concrete shield is parallel to the $\mathrm{XZ}$ plane. To obtain $\mathrm{a}_{\mathfrak{c}}$, the cylindrical components must be transformed "backwards" to the original Cartesian system. Figures $3-10$ through $3-15$ will be useful at this point.

Given the cylindrical coordinates $(r, \phi, z)$ of the scattering volume $d V$, its Cartesian coordinates in the $X^{\prime \prime} Y^{\prime \prime} Z$ " system are

$$
\begin{aligned}
& w_{x}^{\prime \prime}=z, \\
& w_{y}^{\prime \prime}=r \cos \phi, \\
& w_{z}^{\prime \prime}=r \sin \phi .
\end{aligned}
$$




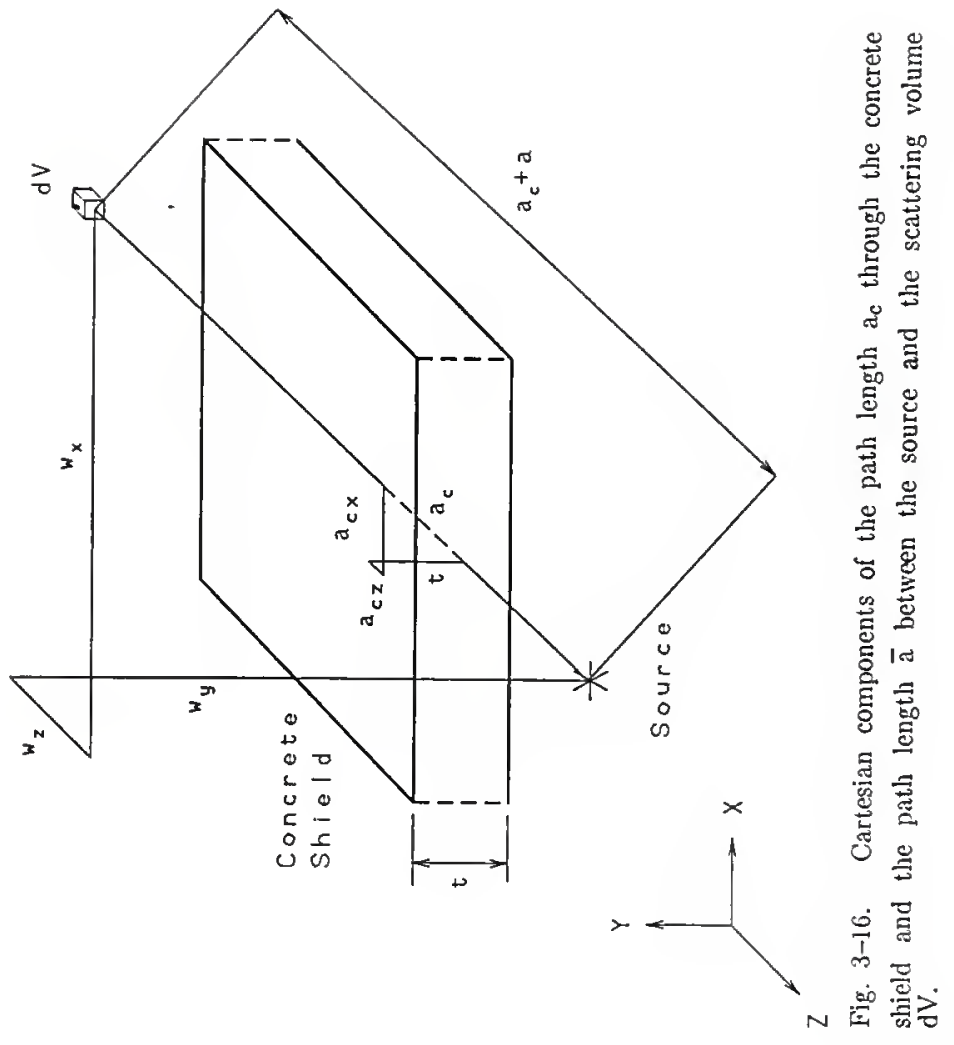


If a Cartesian coordinate system is rotated about its origin to produce a second system, the coordinates of one point in both systems are related to one another by the following system of equations:

$$
\left.\begin{array}{l}
x=\lambda_{x} x^{\prime}+\lambda_{y} y^{\prime}+\lambda_{z} z^{\prime} \\
y=\mu_{x} x^{\prime}+\mu_{y} y^{\prime}+\mu_{z} z^{\prime} \\
z=\nu_{x} x^{\prime}+\nu_{y} y^{\prime}+\nu_{z} z^{\prime}
\end{array}\right\}
$$

where

$$
\begin{aligned}
(\mathrm{x}, \mathrm{y}, \mathrm{z})= & \text { coordinates of the point in } \mathrm{XYZ} \text { space, } \\
\left(\mathrm{x}^{\prime}, \mathrm{y}^{\prime}, \mathrm{Z}^{\prime}\right)= & \text { coordinates of the point in } \mathrm{X}^{\prime} \mathrm{Y}^{\prime} Z^{\prime} \text { space, } \\
\left(\lambda_{x}, \mu_{\mathrm{x}}, \nu_{\mathrm{x}}\right)= & \text { direction cosines of the } \mathrm{X}^{\prime} \text { axis (not the } \\
& \mathrm{X} \text { axis) with respect to the } \mathrm{XY} \mathrm{Y}^{\prime} \text { coordinate } \\
& \text { axes. }
\end{aligned}
$$

This system of equations will yield the coordinates of $d V$ in the $X^{\prime} Y^{\prime} Z^{\prime}$ system, which was rotated about the $Y^{\prime}$ axis through an angle $\xi$ to produce the $X^{\prime \prime} Y " Z "$ axes. With $\lambda_{x}=\cos \xi, \lambda_{y}=0$, and $\lambda_{z}=-\sin \xi$, for example,

$$
w_{x}^{\prime}=z \cos \xi-r \sin \phi \sin \xi .
$$

Similarly,

$$
\begin{aligned}
& \mathrm{w}_{\mathrm{y}}^{\prime}=\mathrm{r} \cos \phi, \\
& \mathrm{w}_{\mathrm{z}}^{\prime}=\mathrm{z} \sin \xi+\mathrm{r} \sin \phi \cos \xi .
\end{aligned}
$$


The same operation can be performed with Eqs. (3-37) through (3-40) to find the coordinates of $d V$ in $X Y Z$ space.

$$
\begin{aligned}
& \mathrm{w}_{\mathrm{x}}=\mathrm{z} \cos \xi \cos \theta-\mathrm{r}(\sin \phi \sin \xi \cos \theta-\cos \phi \sin \theta) \\
& \mathrm{w}_{\mathrm{y}}=-\mathrm{z} \cos \xi \sin \theta+\mathrm{r}(\sin \phi \sin \xi \sin \theta+\cos \phi \cos \theta) \\
& \mathrm{w}_{\mathrm{z}}=\mathrm{z} \sin \xi+\mathrm{r} \sin \phi \cos \xi
\end{aligned}
$$

With this example, we can now determine $a_{c}$, the concrete path length. Because $a_{c}$ lies on a line from the source (the origin) to $d V$, its components ${ }^{a} c, x, a_{c, y}$ and $a_{c, z}$ are proportional to the components $w_{x}, w_{y}$, and $w_{z}$. Further, the $Y$ component must always be equal to $t$, the concrete shield thickness. So, we may define the components of $a_{c}$ as the coordinates of $d V$, normalized to a $\mathrm{Y}$-component of $\mathrm{t}$. Define

$$
\begin{aligned}
& \mathrm{a}_{c, x}=\mathrm{t} \mathrm{w}_{x} / \mathrm{w}_{y}, \\
& { }^{\mathrm{a}}{ }_{c, z}=\mathrm{t} \mathrm{w}_{z} / \mathrm{w}_{y},
\end{aligned}
$$

using Eqs. (3-41) - (3-43), The concrete path length is then given by

$$
a_{c}=\left[a_{c, x}^{2}+a_{c, z}^{2}+t^{2}\right]^{\frac{1}{2}} .
$$

Finally, with the Compton scattering quantities defined by Eqs. (3-4) and $(3-5)$, the exposure rate equation (3-9) may be expressed as an integral over $r, z$ and $\phi$. 


$$
\begin{gathered}
\dot{\mathrm{X}}=\frac{\mathrm{SKN}}{4 \pi} \int_{\mathrm{r}_{\mathrm{m} \text { in }}}^{\mathrm{r}_{\max }} \mathrm{dr} \mathrm{r} \int_{\mathrm{Z}_{\mathrm{m} \text { in }}}^{z_{\max }} \mathrm{dz} \frac{\hat{\mathrm{B}}}{\mathrm{b}^{2}} \frac{\hat{\mu}_{\mathrm{en}}}{\rho} \sigma \hat{\mathrm{E}} \exp (-\hat{\mu} \mathrm{b}) \\
\cdot \int_{\phi_{\min }}^{\phi_{\max }} \mathrm{d} \phi \frac{\mathrm{B}}{\left(\mathrm{a}+\mathrm{a}_{\mathrm{c}}\right)^{2}} \exp \left(-\mu \mathrm{a}-\mu_{\mathrm{c}} \mathrm{a}_{\mathrm{c}}\right)
\end{gathered}
$$

Unlike the equation for the silo geometry, this does not approximate the path length a as independent of the inner variable. For the case of an unshielded source, $a_{c}=0$ and the integrands show no dependence on $\phi$. Equation (3-47) then reduces to

$$
\begin{gathered}
\dot{\mathrm{X}}=\frac{\mathrm{SKN}}{4 \pi} \int_{\mathrm{r}_{\mathrm{m} \text { in }}}^{r_{\mathrm{max}}} \mathrm{dr} \int_{z_{\min }}^{z_{\max }} \mathrm{dz} \frac{\hat{\mathrm{B}}}{\mathrm{a}^{2} \mathrm{~b}^{2}} \frac{\hat{\mu}_{\mathrm{en}}}{\rho} \sigma \hat{\mathrm{E}} \exp (-\mu \mathrm{a}-\hat{\mu} \mathrm{b}) \\
\cdot\left(\phi_{\max }-\phi_{\min }\right) .
\end{gathered}
$$

In this coordinate system, the variables $\sigma, \hat{\mathrm{E}}$ and $\hat{\mu}_{\mathrm{er}} / \rho$ (itself a function of $\hat{E}$ ) again depend on $\beta$, but $\beta$ now depends on the cylindrical coordinates $r$ and 2 . As a result, those variables move from the outer integral to an inner integral and must be evaluated more often. This disadvantage of the cylindrical system is offset by the relative simplicity of the integration limits, which are presented next. 


\subsubsection{Limits of Integration}

The limits of integration in Eq. (3-47) are best understood with the aid of Fig. 3-17. The scattering volume $\mathrm{dV}$ must always lie at a position which is visible to the source and the detector; this is a requirement of the single-scatter model. The union of the regions which are visible from the source and from the detector is the shaded area labeled the "scattering zone" in Fig. 3-17. This volume of space extends infinitely into and out of the page, as the wall does. The differential scattering volume $\mathrm{dV}$ is restricted to the scattering zone, thus integration takes place over this volume.

Limits on $r$. In Fig. $3-18$, the system is oriented so that the scattering zone may be viewed in the cylindrical coordinate system. From this view, limits on $\mathrm{r}$ are easily deduced. Recall from Section 3.4.1 and Fig. 3-14 that the source and detector are both a distance $h$ below the wall in the $X^{\prime \prime} Y^{\prime \prime} Z^{\prime \prime}$ coordinate system. The edge of the wall marks the closest approach of the scattering zone to the source-detector axis, therefore $r_{\min }=h$.

The upper limit of integration over $r$ in fact approaches infinity, since a gamma ray could, in theory, travel an infinite distance, undergo a Compton scatter of $180^{\circ}$, and return again to the detector. As with the numerical solution of the silo problem, however, contributions from scattering volumes very far from the detector are negligible, and a cutoff criterion is necessary. The criterion selected by Roseberry for the SKY code, mean free paths traveled by the photon in air, is satisfactory.

Let $r_{\max }$ be chosen such that for all values of $r>I_{\max }$, a photon must travel a distance iu mean free paths greater than the chosen cutoff value $M_{\text {cut }}$. 


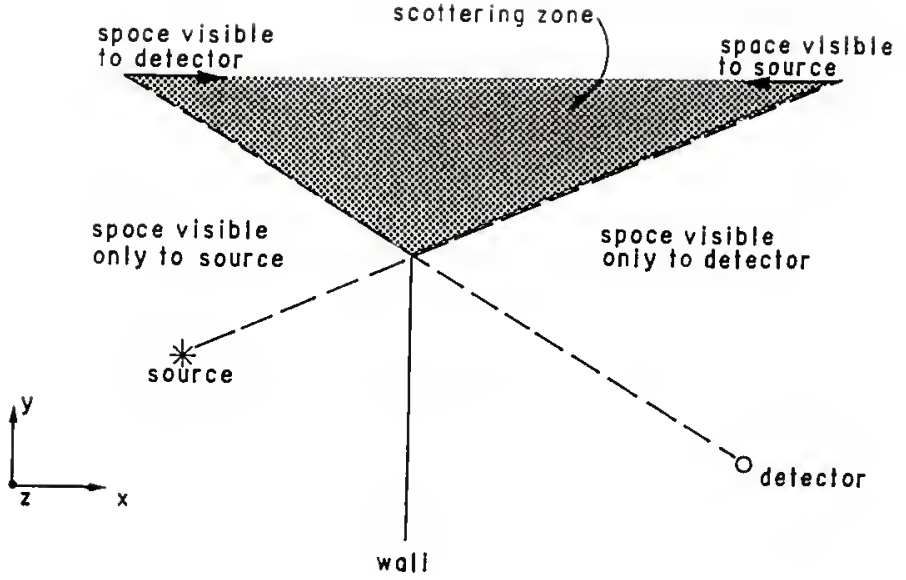

Fig. 3-17. Definition of the scattering zone used to formulate limits of integration for the wall geometry. 


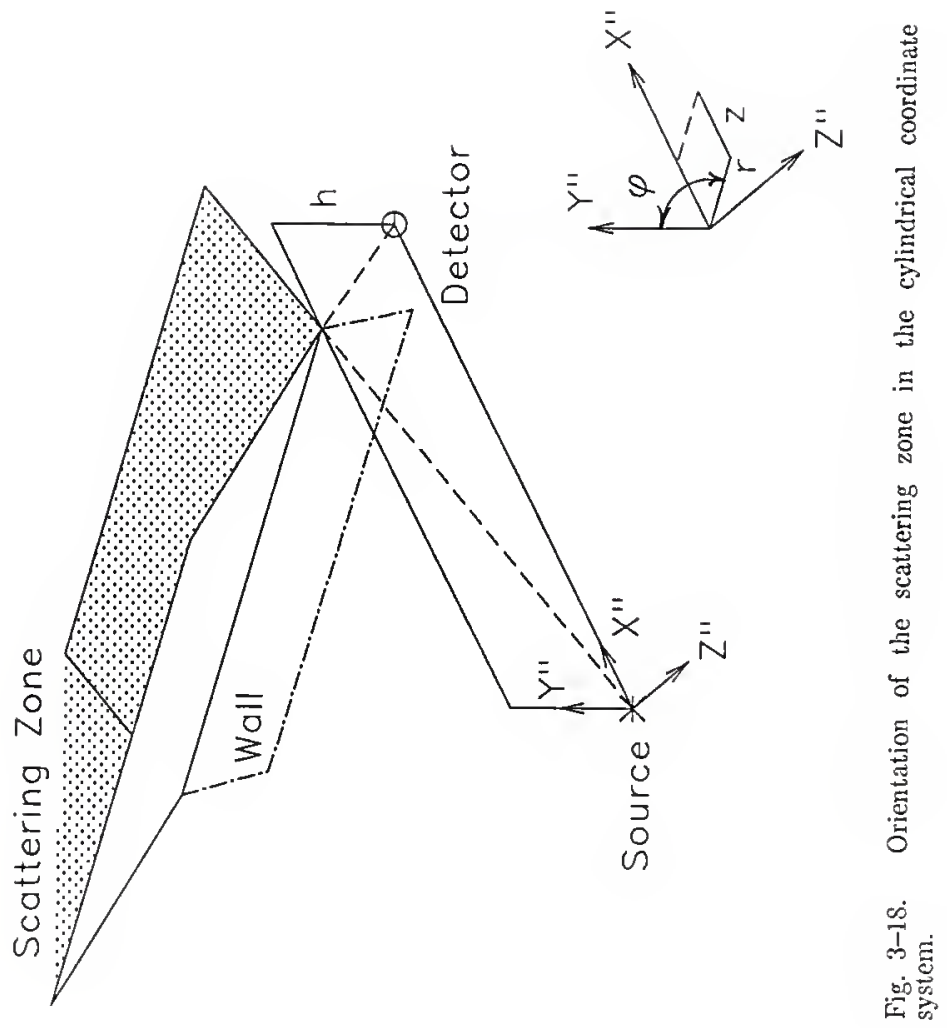


Referring to Fig. 3-4, the distance traveled by the photon in air is $\mu \mathrm{a}+\hat{\mu} \mathrm{b}$. Since the attenuation coefficient of air increases as photon energy decreases over nearly all energies of interest, $\mu<\hat{\mu}$. Define the path length function $\mathrm{M}$ as

$$
\mathrm{M}(\mathrm{z})=\mu \mathrm{a}+\mu \mathrm{b} \approx \mu\left[\left[\mathrm{r}^{2}+\mathrm{z}^{2}\right]^{\frac{1}{2}}+\left[\mathrm{r}^{2}+(\mathrm{d}-\mathrm{z})^{2}\right]^{\frac{1}{2}}\right] .
$$

If $\mathrm{M}$ equals the cutoff value $\mathrm{M}_{\text {cut, }}$, then the actual mean free path distance in air, $\mu \mathrm{a}+\hat{\mu} \mathrm{b}$, will exceed $\mathrm{M}_{\text {cut }}$, since $\mu \mathrm{b}<\hat{\mathrm{b}} \mathrm{b}$. Defining $\mathrm{r}_{\max }$ in terms of $\mathrm{M}$ instead of $\mathrm{M}_{\mathrm{cut}}$ is a conservative choice, insuring that all scattering volumes for which the path length is below $M_{\text {cut }}$ are included in the integration.

By the first derivative test, $M$ is minimum at $z=d / 2$ for constant $\mathrm{r}$. If the minimum value of $\mathrm{M}$ for constant $\mathrm{r}$ is $\mathrm{M}_{\mathrm{cut}}$, the minimum mean free path length in air for that value of $\mathrm{r}$ must be greater than $\mathrm{M}_{\text {cut }}$, since $\mu \mathrm{b}>$ $\mu \mathrm{b}$. This value of $\mathrm{r}$ may be taken as $\mathbf{r}_{\max }$. Substituting $\mathrm{z}=\mathrm{d} / 2$ and $\mathrm{M}=$ $\mathrm{M}_{\text {cut }}$,

$$
M_{\text {cut }}=\mu\left[\sqrt{r^{2}+(d / 2)^{2}}+\sqrt{r^{2}+(d-d / 2)^{2}}\right]
$$

or

$$
\mathrm{r}_{\text {max }}=\left[\frac{1}{2}\right]\left[\left(\mathrm{M}_{\text {cut }} / \mu\right)^{2}-\mathrm{d}^{2}\right] .
$$

This formula is used in the numerical analysis for the wall case.

Limits on $z$. The limits on $z$ and $\phi$ are strongly dependent on the source and detector locations as measured from the wall. The possible combinations of source and detector positions with respect to the wall may be 
divided into three categories. These categories, shown in Fig. 3-19, are visualized best in the $\mathrm{X}^{\prime \prime} \mathrm{Y}^{\prime \prime} \mathrm{Z}$ " frame of reference, with the source-detector axis horizontal. They are:

(1) The wall lies between the source and detector, and is not directly above either. This occurs only when $x_{s}^{\prime \prime}>0$ and $x_{d}^{\prime \prime}>0$.

(2) The wall extends over and above the source in the $Y$ "direction; in the $\mathrm{XYZ}$ system, the detector is much farther below the top of the wall than the source. In this case, $x_{S}^{\prime \prime} \leq 0$.

(3) The wall extends over and above the detector in the $Y$ " direction; in the XYZ system, the source is much farther below the top of the wall than the detector. In this case, $x_{d}^{\prime \prime} \leq 0$.

The second and third cases may be useful in modeling situations where a source and detector are at the base of a cliff and top of the cliff, or at the base and roof of a tall building.

For a specific value of $r$, the scattering volume $d V$ will lie on the surface of a cylinder of radius $\mathrm{r}$ about the source-detector axis. If any portion of the cylinder lies within the scattering zone of Fig. 3-17, that value of $r$ lies within the limits of integration. Some examples are illustrated in Fig. 3-20. The smallest useful cylinder, of course, has radius $r_{\min }=h$. For a cylinder of radius $r>h$, the values of $z$ between which the cylinder intersects the scattering zone correspond to the analytical limits of integration for $z$. The problem is therefore reduced to locating the intersection of the cylinder and the two half-planes bordering the scattering zone. Restrictions on $z$ based on the mean-free-path cutoff $\mathrm{M}_{\text {cut }}$ will also be required. 


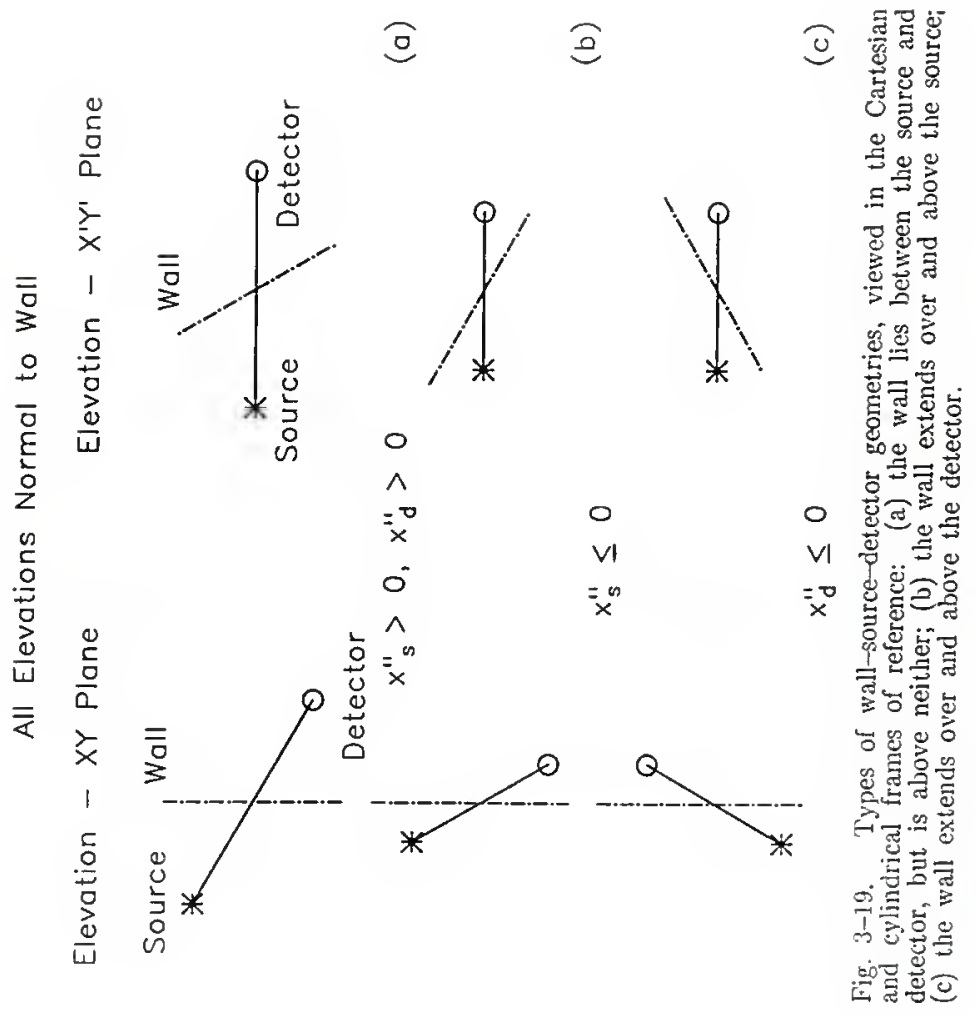



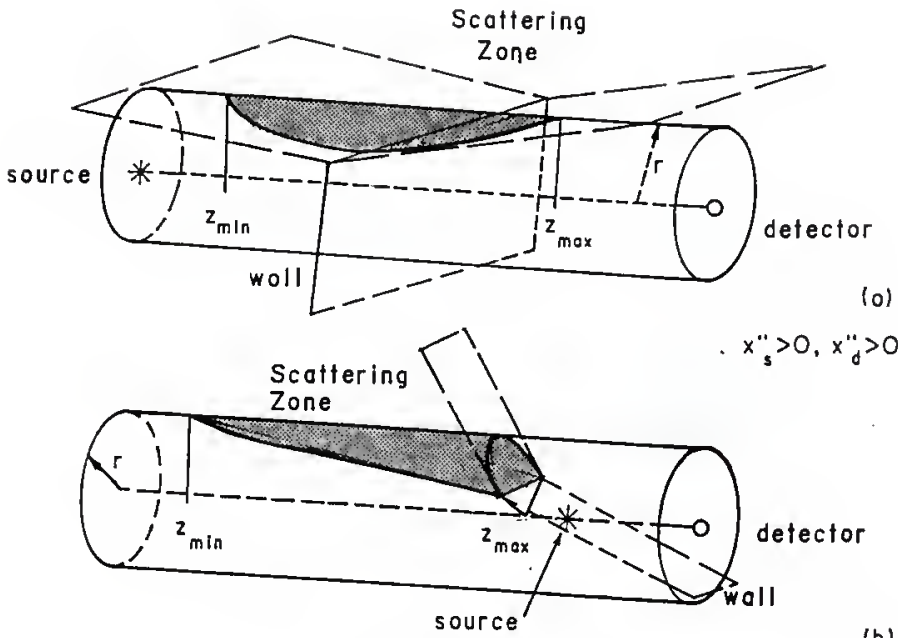

(b)

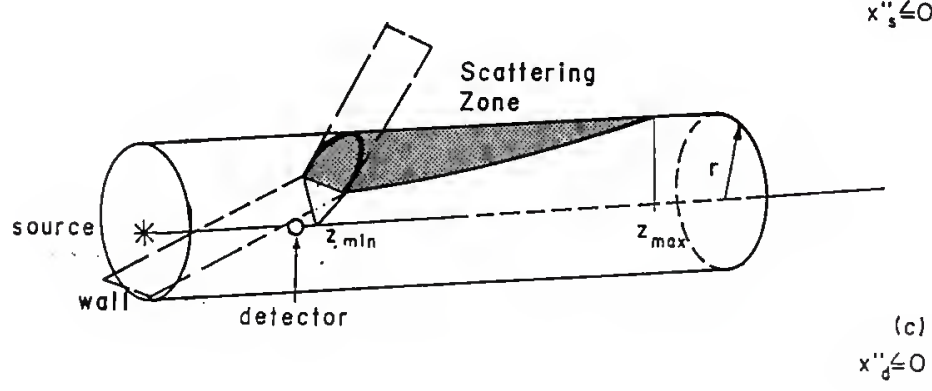

Fig. 3-20. Cylindrical surfaces used to determine limits of integration on z: (a) the wall lies between the source and detector, but is above neither; (b) the wall extends over and above the source; (c) the wall extends over and above the detector. 
To begin, consider the first geometry $\left(x_{S}^{\prime \prime}>0, x_{d}^{\prime \prime}>0\right)$ illustrated in Fig. $3-20$. At $z=z_{\min }$, the cylinder contacts the scattering zone at only one point: the half-plane above the source, which passes through the top edge of the wall and defines the space visible from the detector. The radial $r$ will be perpendicular to this half-plane at $z=z_{\min }$, since $r$ defines a circle which is tangent to the plane. This fact can be utilized to compute $z_{\text {min }}$. A skeletal geometry, with only the wall edge, half-plane above the source, and the source-detector axis, is shown in Fig. 3-21. From this figure, Eq. (3-29) and the identity $\mathrm{d}=\mathrm{x}_{\mathrm{S}}^{\prime \prime}+\mathrm{x}_{\mathrm{d}}^{\prime \prime}$,

$$
\begin{aligned}
\alpha & =\arctan \frac{\mathrm{h}}{\mathrm{x}_{\mathrm{d}}^{\prime \prime} \cos \xi}=\arctan \frac{\mathrm{h}}{\mathrm{x}_{\mathrm{d}}^{\prime}}, \\
\mathrm{p} & =(\mathrm{d}-\mathrm{z}) \cos \xi \tan \alpha=\mathrm{h}(\mathrm{d}-\mathrm{z})(\cos \xi) / \mathrm{x}_{\mathrm{d}}^{\prime}, \\
\gamma & =\pi / 2-\xi \\
\tan \delta & =\frac{\tan \alpha}{\sec \gamma}=\frac{\mathrm{h}}{\mathrm{x}_{\mathrm{d}}^{\prime} \csc \xi},
\end{aligned}
$$

and

$$
\mathrm{r}=\mathrm{p} \cos \delta=\frac{\mathrm{h}(\mathrm{d}-\mathrm{z}) \cos \xi}{\left(\mathrm{h}^{2} \sin ^{2} \xi+\mathrm{x}_{\mathrm{d}}^{2}\right)^{\frac{1}{2}}} .
$$

Rearranging Eq. (3-56) to produce $z$ as a function of $r$, an expression is obtained for the lower limit on $\mathrm{z}$.

$$
z=d-\frac{r\left(h^{2} \sin ^{2} \xi+x_{d}^{\prime}\right)^{\frac{1}{2}}}{h \cos \xi} .
$$




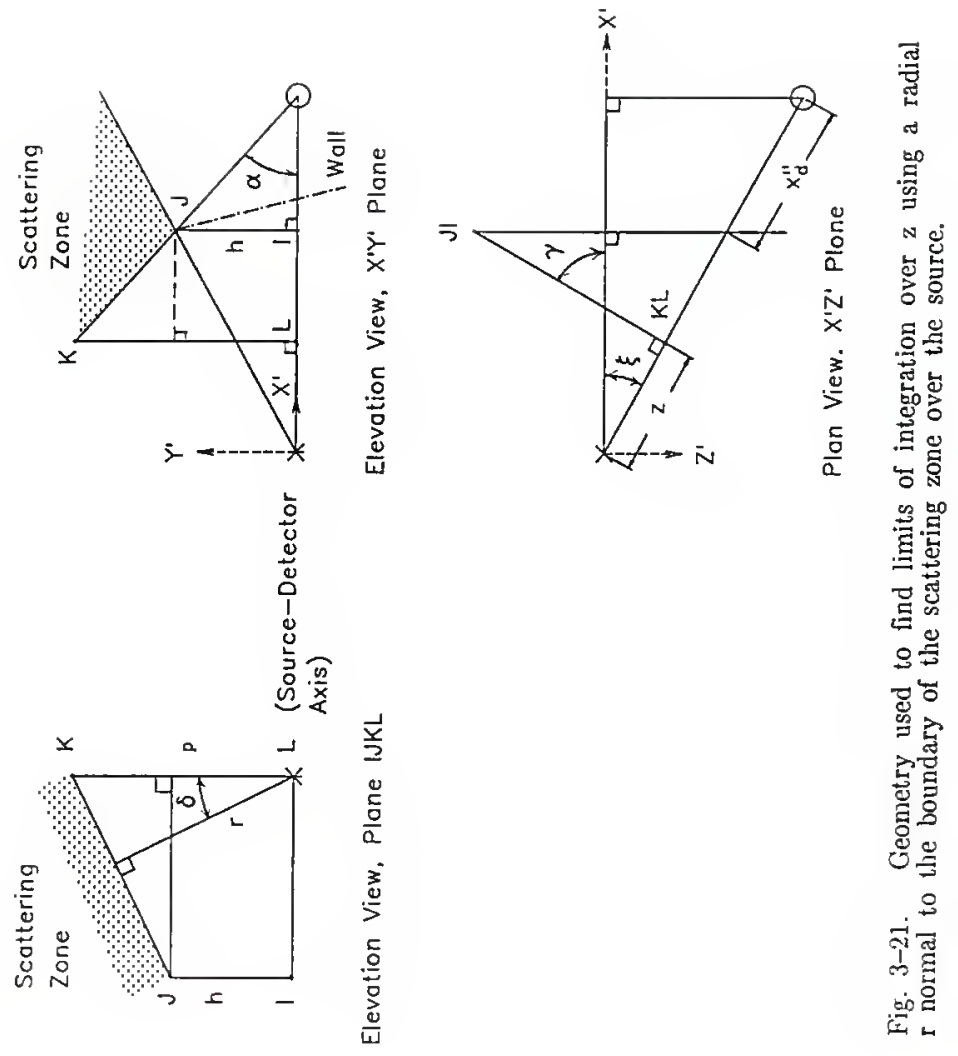


Figure 3-22 shows a similar skeletal geometry for the half-plane above the detector, which marks the space visible from the source. At $z=z_{\max }$, the cylinder in Fig. 3-20(a) intersects this half-plane at only one point. By identical analysis, the value of $z$ on the detector side, $z_{\max }$, is given by

$$
z=\frac{r\left(h^{2} \sin ^{2} \xi+x_{s}^{\prime}\right)^{\frac{1}{2}}}{h \cos \xi} .
$$

For all $r$ such that a radial of length $r$ exists normal to the half-plane above the source, Eq. (3-57) gives the value of $z_{\min }$. If $\xi \neq 0$, however, there can be values of $z$ for which no radial from the axis to the scattering zone is perpendicular to the half-plane above the source, because the half-plane does not extend to those values of $z$. Figure 3-23 demonstrates that all radials which are normal to the half-plane lie in a plane of their own. The important value of $\mathrm{r}$ is that value for which the radial is normal to the half-plane at the edge of the wall. For larger r, Eq. (3-57) yields $z_{\min }$. For smaller $r$, the radial at $(r, z)$ which contacts the half-plane at only one point does so at the top of the wall, and this fact must be used to find $z_{\min }$. The same argument applies to Eq. (3-58); if $\mathrm{r}$ is greater than or equal to the length of the radial which reaches the edge of the wall and is normal to the half-plane over the detector, Eq. (3-58) produces $z_{\max }$. Otherwise, $z_{\max }$ is determined differently.

Figure 3-24 presents a typical radial of length $r$, at axial position $z$, which ends at the top of the wall. The radial acts as the diagonal of a box, and its length may easily be computed. From the figure, 

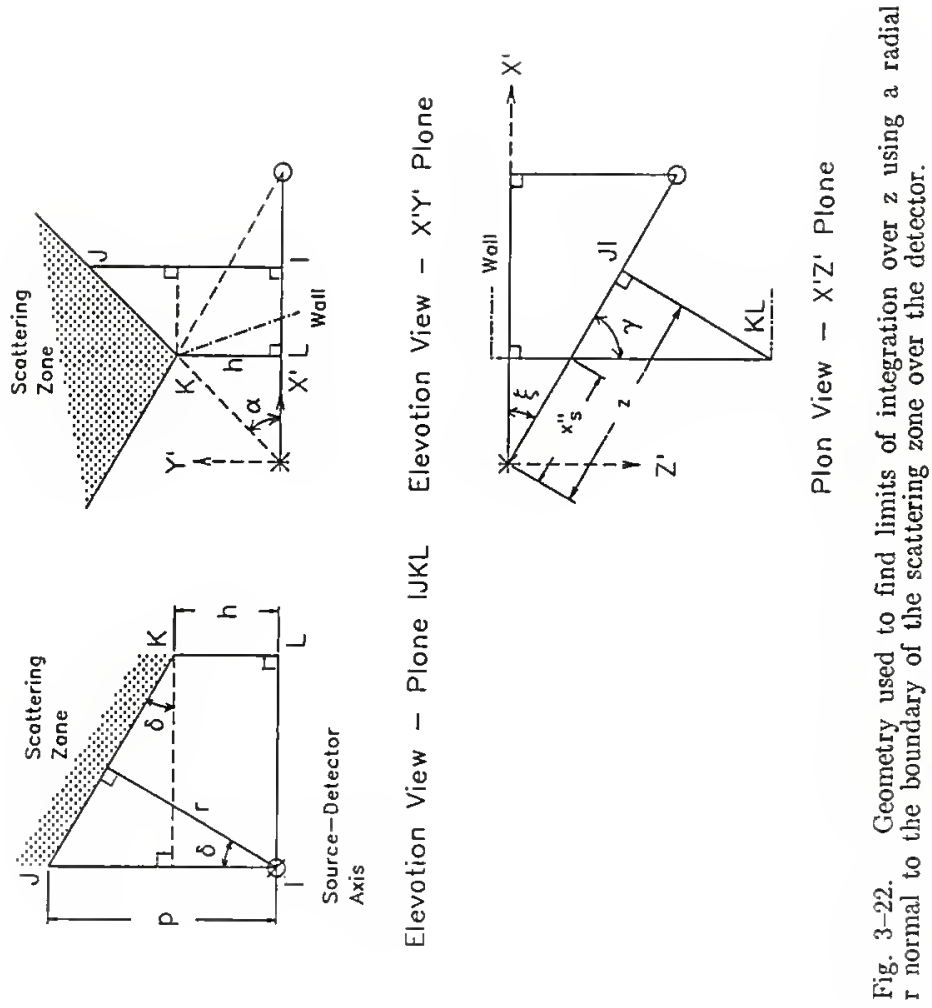


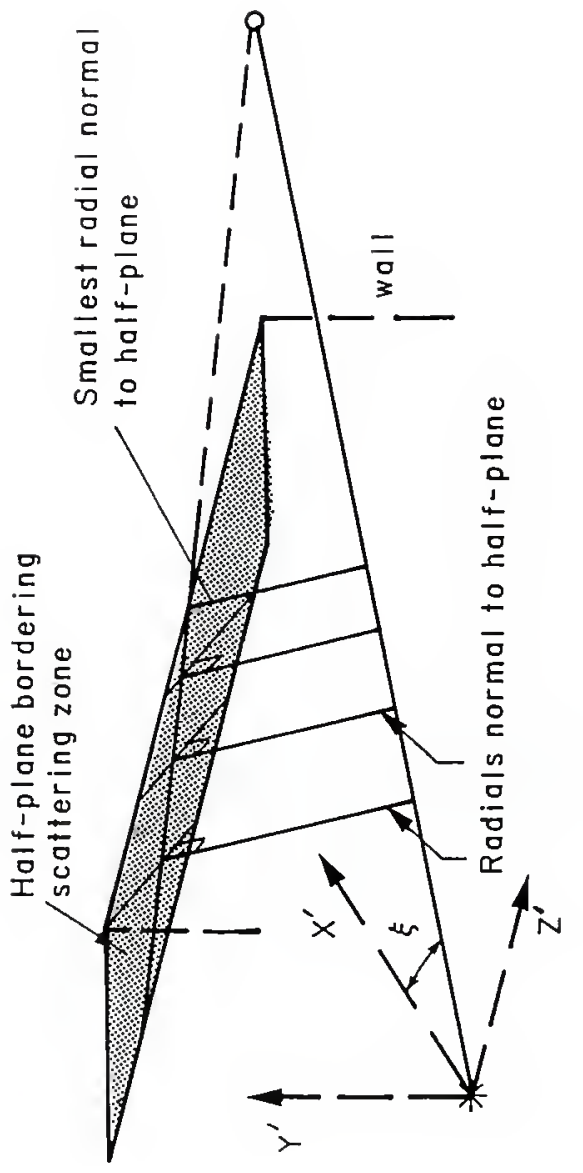

总 

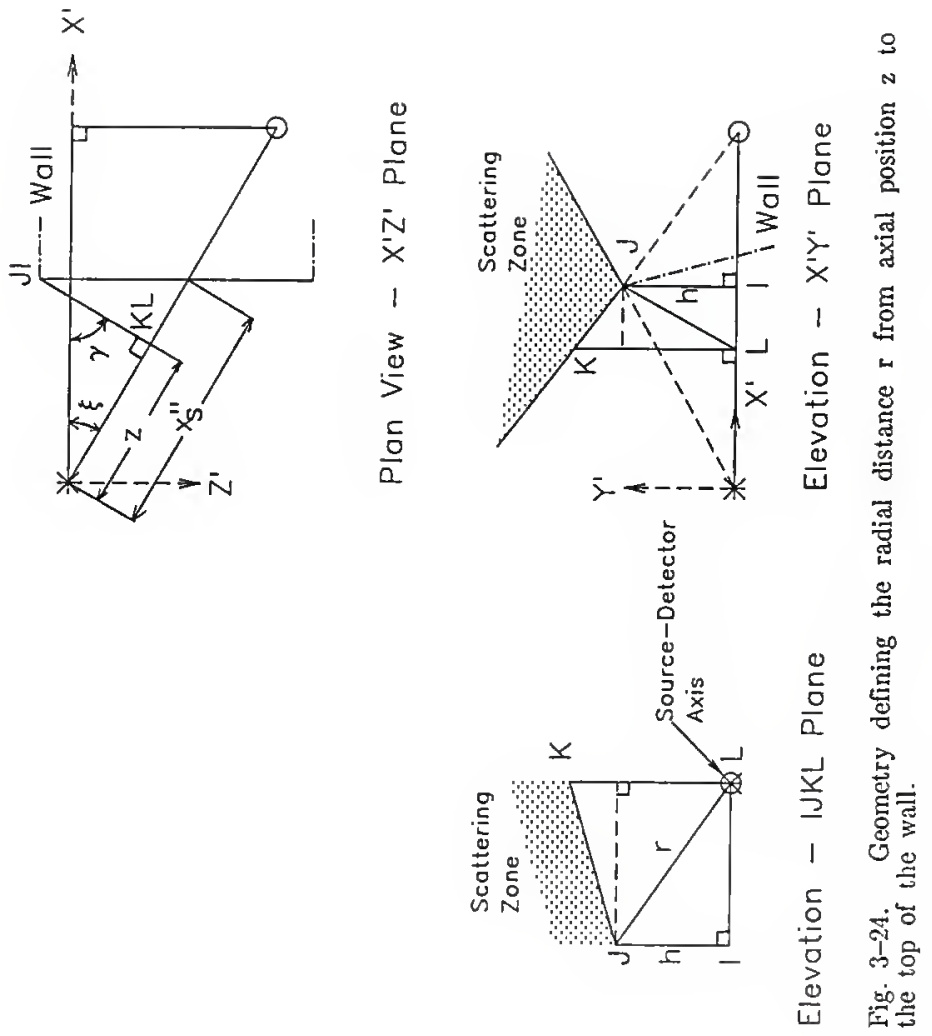


$$
\mathrm{r}^{2}=\mathrm{h}^{2}+\left[\left(\mathrm{x}_{\mathrm{S}}^{\prime \prime}-\mathrm{z}\right) \cos \xi\right]^{2}+\left[\left(\mathrm{x}_{\mathrm{S}}^{\prime \prime}-\mathrm{z}\right) \cos \xi \tan \gamma\right]^{2}
$$

Using Eqs. (3-28) and (3-54) and the identities $\tan \gamma=\cot (\pi / 2-\gamma)$ and $1+\cot ^{2} \xi=\csc ^{2} \xi$

$$
\mathrm{r}^{2}=\mathrm{h}^{2}+\left(\mathrm{x}_{\mathrm{s}}^{1}-\mathrm{z} \cos \xi\right)^{2} \csc ^{2} \xi
$$

To add the requirement that the same radial must be normal to the half-plane over the source, Eq. $(3-57)$ is substituted into Eq. (3-60); the result is the minimum value of $\mathrm{r}$ for which $\mathrm{Eq}$. (3-57) equals $\mathrm{z}_{\mathrm{min}}$.

$$
r=\frac{h}{x_{d}^{T}}\left[h^{2} \sin ^{2} \xi+\left(x_{d}^{\prime}\right)^{2}\right]^{\frac{1}{2}}
$$

Substituting Eq. (3-58) into (3-60) yields the smallest radial distance $r$ for which Eq. (3-58) equals $z_{\max }$.

$$
r=\frac{h}{x_{S}^{1}}\left[h^{2} \sin ^{2} \xi+\left(x_{S}^{\prime}\right)^{2}\right]^{\frac{1}{2}} .
$$

For instances in which $r$ is less than one or both of the criteria above, Eq. (3-60) may be used alone to find the appropriate limit on $z$. By solving for $z$ without the use of Eqs. $(3-57)$ or $(3-58)$, the restriction that the radial be normal to a half-plane is lifted. Equation $(3-60)$ has two solutions for $z$, roots of a quadratic. 


$$
\left.\begin{array}{l}
z_{1}=\left[x_{S}^{\prime}-\sin \xi\left(r^{2}-h^{2}\right)^{\frac{1}{2}}\right] \sec \xi \\
z_{2}=\left[x_{s}^{\prime}+\sin \xi\left(r^{2}-h^{2}\right)^{\frac{1}{2}}\right] \sec \xi
\end{array}\right\}
$$

The smaller of the two roots corresponds to $z_{\text {min }}$ the larger corresponds to $z_{\max }$. Either or both may be used as a limit, as the geometry demands.

To summarize the analysis to this point: The limits on $z$ are piecewise functions, with the point of changeover dependent on r. For the case of $x_{s}^{\prime \prime}>$ 0 and $x_{d}^{\prime \prime}>0$

$$
\begin{aligned}
& z_{\min }= \begin{cases}d-r \frac{\left[h^{2} \sin ^{2} \xi+\left(x_{d}^{\prime}\right)^{2}\right]^{\frac{1}{2}}}{h \cos \xi}, r \geq \frac{h}{x_{d}^{r}}\left[h^{2} \sin ^{2} \xi+\left(x_{d}^{\prime}\right)^{2}\right]^{\frac{1}{2}} \\
\min \left(z_{1}, z_{2}\right) & \text {, otherwise. }\end{cases} \\
& z_{\text {max }}= \begin{cases}r \frac{\left[h^{2} \sin ^{2} \xi+\left(x_{S}^{\prime}\right)^{2}\right]^{\frac{1}{2}}}{h \cos \xi}, r \geq \frac{h}{x_{S}^{r}}\left[h^{2} \sin ^{2} \xi+\left(x_{S}^{\prime}\right)^{2}\right]^{\frac{1}{2}} \\
\max \left(z_{1}, z_{2}\right) & , \text { otherwise. }\end{cases}
\end{aligned}
$$

The second and third geometry cases in Figs. 3-19 and 3-20 may now be discussed. In the second class of problems, where $x_{S}^{\prime \prime} \leq 0$ and $x_{d}^{\prime \prime}>0$, both half-planes bordering the scattering zone lie above and behind the point source in the cylindrical frame of reference. The guidelines for determining $\mathrm{z}_{\text {min }}$ are the same as for the first class of problems, since the half-plane on 
which $z_{\text {min }}$ depends still extends above and behind the source in these cases. The guidelines for determining $z_{\max }$, however, must be changed. When $\mathrm{z}=$ $z_{\max }$, the cylindrical surface of radius $\mathrm{r}$ intersects the scattering zone at only one point. As shown in Fig. 3-20(b), this point is the very tip of the wedgeshaped scattering zone, which is also the top of the wall. The radial I and its position $z$ are thus governed by Eq. (3-60), and $z_{\max }$ equals the larger of its two solutions in Eq. (3-63). Figure 3-20(c) presents the case of $x_{s}^{\prime \prime}>0$, $x_{\mathrm{d}}^{\prime \prime} \leq 0$, wherein both half-planes extend behind the detector. By analogy, we find $z_{\min }$ is always the smaller of the solutions to $\mathrm{Eq}$. $(3-60)$, while $z_{\max }$ follows the same guidelines as in the first class of problem geometries.

Computing limits of integration on $z$ using only the half-planes bordering the scattering zone will lead to inefficiency; on occasion, much of the cylinder of radius $\mathrm{r}$ between these values of $\mathrm{z}$ will lie further from the source and detector than a practical value of the path length cutoff $\mathrm{M}_{\text {cut }}$. An example is that of large source-detector distances and a relatively short wall. Integrating over this space yields a negligible response, and therefore is unnecessary. Alternate limits of integration over $z$ based on the path length cutoff $M_{\text {cut }}$ will avoid this wasted effort.

Figure 3-25 illustrates an approximation for useful limits on $z$ using the mean free path cutoff. Since the total path length traveled by a photon is restricted to $M_{\text {cut }}$ mean free paths, the photon must scatter within the ellipsoid shown. Recall that in two dimensions, the sum of the distances from any point on an ellipse to both foci is a constant. In the figure, this distance is $R_{1}+R_{2}$. By equating this constant sum to the cutoff distance $M_{\text {cut }} / \mu$ for photons at the source energy, and rotating the ellipse about the 


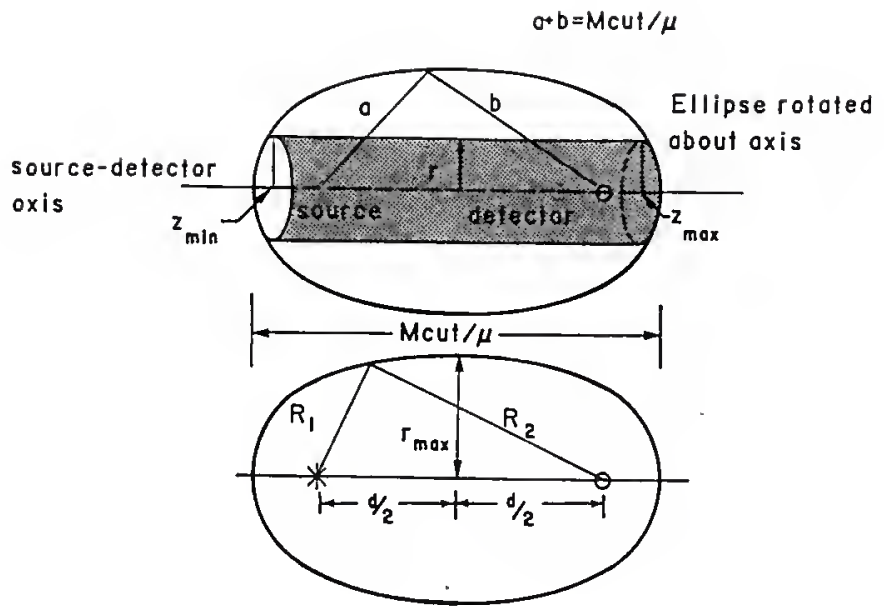

Fig. 3-25. Alternate limits of integration over $z$, based on the mean-free-path cutoff. 
source-detector axis, an ellipsoid is created with major axis of length $\mathrm{M}_{\text {cut }} / \mu$ and minor axis of length $2 \mathrm{r}_{\max }$. This ellipsoid may be used to restrict the range of integration of $z$ to useful values.

In the cylindrical coordinate system, the source rests at the origin, and the detector lies at $r=0, z=d$. The center of the ellipsoid then lies at $\mathrm{r}=0, z=\mathrm{d} / 2$. The analytic equation for the two-dimensional ellipse is

$$
\frac{(\mathrm{z}-\mathrm{d} / 2)^{2}}{\left(M_{\text {cut }} / 2 \mu\right)^{2}}+\frac{(\mathrm{r}-0)^{2}}{\mathrm{r}_{\max }^{2}}=1
$$

If $r$ is known, Eq. (3-66) yields two solutions for $z$ :

$$
\mathrm{z}=\frac{\mathrm{d}}{2} \pm \frac{\mathrm{M}_{\mathrm{cut}}}{2 \mu} \sqrt{1-\left[\frac{\mathrm{r}}{\mathrm{r}_{\mathrm{max}}}\right]^{2}}
$$

Turning to the three-dimensional case, if $r$ is known, the differential scattering volume $d V$ will lie on a cylinder of radius $r$ about the source-detector axis for any $z$ and $\phi$. The intersections of this cylinder with the ellipsoid mark upper and lower limits of $z$ required by the mean free path cutoff. The limiting values correspond to Eq. (3-67), since the cylinder and ellipsoid are invariant in $\phi$.

$$
\begin{aligned}
& z_{\min }^{c u t}=\frac{d}{2}-\frac{M_{\text {cut }}}{2 \mu} \sqrt{1-\left[\frac{\mathrm{r}}{r_{\max }}\right]^{2}} \\
& z_{\max }^{c u t}=\frac{d}{2}+\frac{M_{\text {cut }}}{2 \mu} \sqrt{1-\left[\frac{\mathrm{r}}{r_{\max }}\right]^{2}}
\end{aligned}
$$


The limits obtained with the cutoff value should be compared to the limits on $\mathrm{z}$ obtained using the half-planes bordering the scattering zone. Using the two requirements that photons must collide with air volumes in the scattering zone and travel less than $\mathrm{M}_{\text {cut }}$ mean free paths, the larger of the two values of $z_{\min }$ should be used, and the smaller of the two values of $z_{\max }$ should be applied.

The final form for the limits of integration over $z$ is similar to Eqs. $(3-64)$ and $(3-65)$. The lower limit $z_{\min }$ may be found by Eq. $(3-57)$ only if $x_{\mathrm{d}}^{\prime \prime}>0$; comparing the three types of source-detector geometries will make this evident. If $x_{d}^{\prime \prime} \leq 0$ or the radial coordinate $r$ is smaller than its criterion of Eq. (3-61), $z_{\min }$ will be the smaller of $z_{1}$ and $z_{2}$, given by Eq. (3-63). If the value $z_{\text {min }}^{\text {cut }}$ from the mean free path cutoff analysis is larger in any instance, it becomes $z_{\min }$ instead. The upper limit follows similar guidelines involving $x_{S}^{\prime \prime}$. For all possible cases, then, the limits of integration over $z$ are expressed by the following formulas.

$$
z_{\min }=\max \left(z_{\min }^{\text {geom }}, z_{m i n}^{c u t}\right),
$$

where

$$
z_{\min }^{\text {geom }}= \begin{cases}d-r \frac{\sqrt{h^{2} \sin ^{2} \xi+\left(x_{d}^{\prime}\right)^{2}}}{h \cos \xi}, & x_{d}^{\prime \prime}>0 \text { and } r \geq \frac{h}{x_{d}^{\prime}} \sqrt{h^{2} \sin ^{2} \xi+\left(x_{d}^{\prime}\right)^{2}} \\ \min \left(z_{1}, z_{2}\right) & , \text { otherwise }\end{cases}
$$




$$
\begin{aligned}
& z_{\min }^{\text {cut }}=\frac{d}{2}-\frac{M_{c u t}}{2 \mu} \sqrt{1-\left[\frac{r}{r_{\max }}\right]^{2}} . \\
& z_{\max }=\min \left[z_{\max }^{\text {geom }}, z_{\max }^{\text {cut }}\right],
\end{aligned}
$$

where

$$
z_{\max }^{\text {geom }}= \begin{cases}r \frac{\sqrt{h^{2} \sin ^{2} \xi+\left(x_{S}^{\prime}\right)^{2}}}{h \cos \xi}, & x_{s}^{\prime \prime}>0 \text { and } r \geq \frac{h}{x_{s}^{1}} \sqrt{h^{2} \sin ^{2} \xi+\left(x_{S}^{\prime}\right)^{2}} \\ \max \left(z_{1}, z_{2}\right) & , \text { otherwise, }\end{cases}
$$

and

$$
{ }_{\max }^{c u t}=\frac{d}{2}+\frac{M_{c u t}}{2 \mu} \sqrt{1-\left[\frac{\mathrm{r}}{\mathrm{r}_{\max }}\right]^{2}} .
$$

The quantities $z_{1}$ and $z_{2}$ are defined by Eq. (3-63). In a case where the source and detector are not offset, $z=\xi=0$, and the limits simplify greatly.

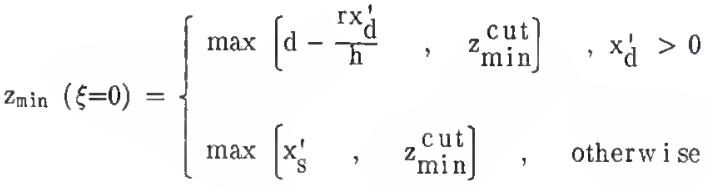

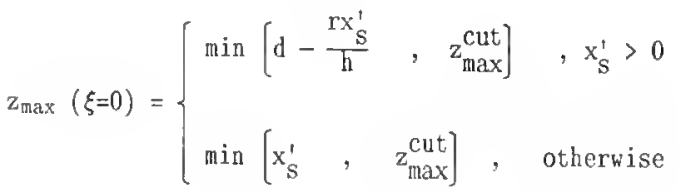


Because the restrictions on $r$ simplify to $r \geq h$, and $r_{\min }=h$, they may be removed entirely.

Limits on $\phi$. The limits of integration over $\phi$ may also be different for each of the three types of source-detector arrangements in Fig. 3-19. The three cases will be considered separately, then combined to produce a single set of equations for $\phi_{\min }$ and $\phi_{\max }$. As will be shown, the region of integration in $\phi$ can sometimes be composed of two regions, with a finite set of values of $\phi$ between them wherein the integral is invalid.

First, consider the case where $x_{s}^{\prime \prime}>0$ and $x_{d}^{\prime \prime}>0$, Fig. 3-19(a). For known $\mathrm{r}$ and $\mathrm{z}$, the scattering volume $\mathrm{dV}$ may reside anywhere on the perimeter of a circle of radius $\mathrm{r}$, with center at distance $z$ along the axis from the point source. Some examples for each type of source-detector arrangement are presented in Fig. 3-26. Only the arc of the circle which lies in the scattering zone may be included in the integration. Thus, the values of $\phi$ which border the arc are the integration limits.

Figure $3-27$ is a skeletal geometry in which a radial $\mathrm{r}$ at position 2 contacts the half-plane over the point source, but may or may not be perpendicular to the plane. Two radials of length $\mathrm{r}$ at two azimuthal angles $\phi$ are possible solutions; one corresponds to $\phi_{\min }$, the other to $\phi_{\max }$. It is possible to employ the length of perpendiculars $m$ and $n$ in computing the limiting angles. Recall that $\phi_{\min }$ and $\phi_{\max }$ are measured from the plane formed by the $\mathrm{Y}^{\prime \prime}$ axis and the source-detector axis (see Fig. 3-15). Also, m, $\mathrm{n}$ and the $\phi$ reference plane are all normal to the $\mathrm{X}^{\prime} \mathrm{Z}$ ' plane. Therefore, $\mathrm{m}$ 

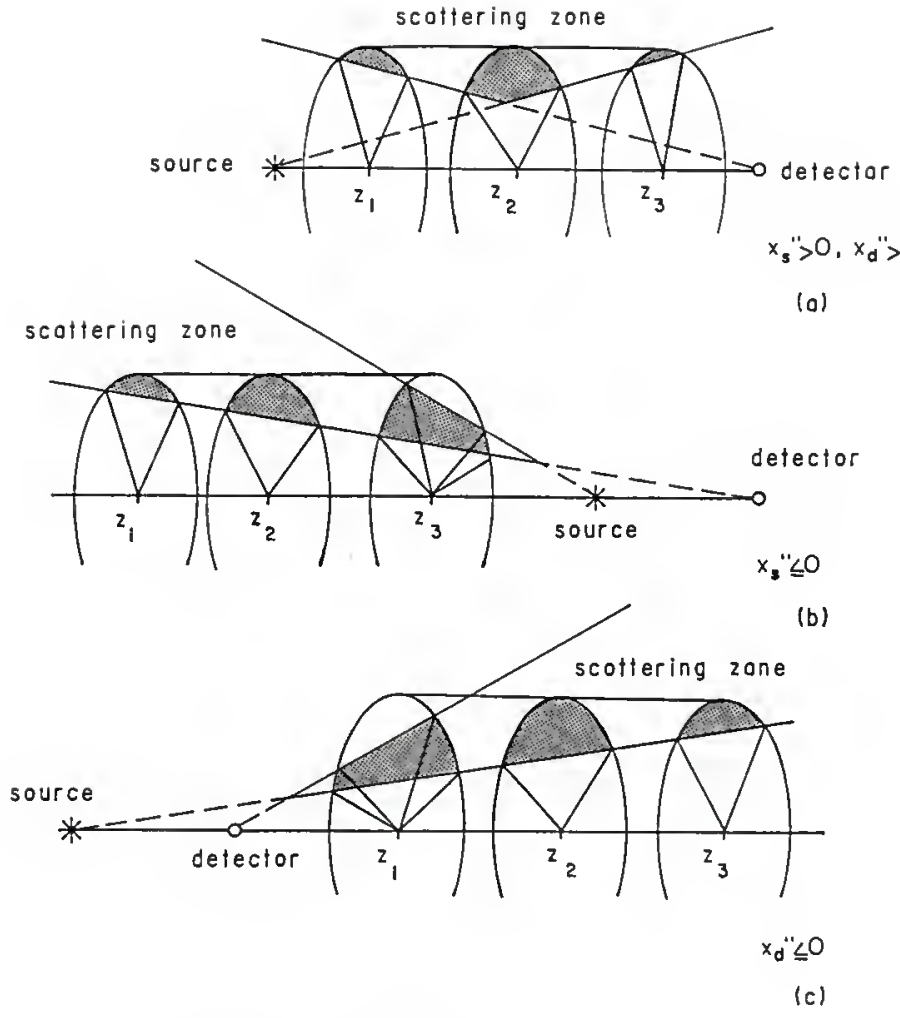

Elevolion views, $X^{\prime} Y$ ' plone, $\xi=30^{\circ}$

Fig. 3-26. Cylindrical surfaces defining limits of integration over $\phi$ for known $\mathrm{r}$ and $\mathrm{z}$ : (a) the wall lies between the source and detector, but is above neither; (b) the wall extends over and above the source; (c) the wall extends over and above the detector. 

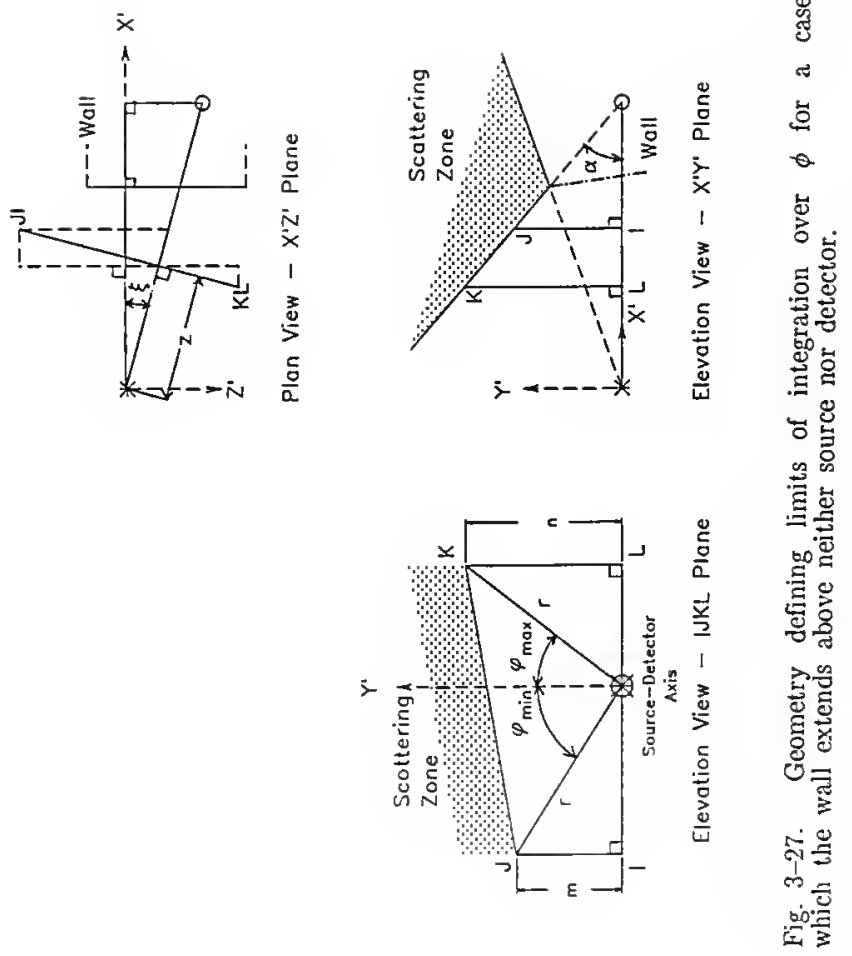
and $\mathrm{n}$ form angles $\phi_{\min }$ and $\phi_{\max }$ with the two radials, respectively, which form the same angles with the $\phi$ reference plane.

From the figure, note that $\phi_{\min }<0$.

$$
\begin{aligned}
\cos \phi_{\text {min }} & =\mathrm{m} / \mathrm{r}, \\
\cos \phi_{\max } & =\mathrm{n} / \mathrm{r}, \\
\tan \alpha & =\frac{\mathrm{m}}{(\mathrm{d}-\mathrm{z}) \cos \xi+\mathrm{rin} \phi_{\min } \sin \xi}, \\
\tan \alpha & =\frac{\mathrm{n}}{(\mathrm{d}-\mathrm{z}) \cos \xi+\mathrm{r} \sin \phi_{\max } \sin \xi} .
\end{aligned}
$$

Combining these with Eq. (3-52) results in a single formula, which may be solved for either limiting value of $\phi$.

$$
\frac{h}{x_{d}^{T}}=\frac{r \cos \phi_{1 i m}}{(d-z) \cos \xi+r \sin \phi_{\mathrm{lim}} \sin \xi} .
$$

The two solutions stem from the presence of $\cos \phi_{1 \text { im }}$ in the numerator and $\sin \phi_{\mathrm{I} \text { im }}$ in the denominator; rearranging Eq. (3-82) yields

$$
h\left[(d-z) \cos \xi+r \sin \phi_{1 i m} \sin \xi\right] / x_{d}^{\prime}=r\left[1-\sin ^{2} \phi_{1 i m}\right]^{\frac{1}{2}} .
$$

Solving for the limits yields

$$
\sin \phi_{\mathrm{Iim}}=\frac{\mathscr{b} \pm \mathscr{B}}{\mathscr{B}}
$$

where

$$
\mathscr{L}=-\mathrm{h}^{2} \sin \xi(\mathrm{d}-2) \cos \xi,
$$




$$
\mathscr{D}=\left\{\left(\mathrm{r} \mathrm{x}_{\mathrm{d}}^{\prime}\right)^{2}\left[\left(\mathrm{x}_{\mathrm{d}}^{\prime}\right)^{2}+(\mathrm{l} \sin \xi)^{2}\right]-\left[\mathrm{h} \mathrm{x}_{\mathrm{d}}^{\prime}(\mathrm{d}-\mathrm{z}) \cos \xi\right]^{2}\right\}^{\frac{1}{2}},
$$

and

$$
\mathscr{C}=\mathrm{r}\left[\left(\mathrm{x}_{\mathrm{d}}^{\prime}\right)^{2}+(\mathrm{h} \sin \xi)^{2}\right] .
$$

The minimum $\phi$ is found by subtracting $\mathscr{B}$ in Eq. (3-84); maximum $\phi$ is found by adding $\mathscr{S}$

This same analysis may be performed for radials which contact the half-plane ahove the detector. The values of $\phi$ at which the circle of radius $r$ and axial position $z$ intersect this half-plane are stated without proof, but are arrived at by the same logic.

$$
\sin \phi_{1 \text { in }}=\frac{\mathscr{D} \pm \mathscr{E}}{\mathscr{F}^{\prime}}
$$

where

$$
\begin{aligned}
& \mathscr{D}=\mathrm{h}^{2} \mathrm{z} \cos \xi \sin \xi, \\
& \mathscr{E}=\left\{\left(\mathrm{rx}_{\mathrm{S}}^{\prime}\right)^{2}\left[\left(\mathrm{x}_{\mathrm{S}}^{\prime}\right)^{2}+(\mathrm{h} \sin \xi)^{2}\right]-\left[\mathrm{hzx_{ \textrm {S } } ^ { \prime }} \cos \xi\right]^{2}\right\}^{\frac{1}{2}},
\end{aligned}
$$

and

$$
\mathscr{F}=\mathrm{r}\left[\left(\mathrm{x}_{\mathrm{S}}^{\prime}\right)^{2}+(\mathrm{h} \sin \xi)^{2}\right] .
$$

The lower limit of $\phi$ is ohtained hy subtracting $\mathscr{E}$ from $\mathscr{D}$, the upper limit hy adding it.

Equations (3-84) through (3-91) are valid only so long as the circle descrihed hy $I$ and $z$ intersects the half-plane with which the equation is derived. In Fig. 3-26(a), one circle at $z_{2}$ straddles the two half-planes, entering the scattering zone through one and leaving out the other. From this case, it may be concluded that a change in expressions for $\phi_{\min }$ and $\phi_{\max }$ 
occurs over the range of $z$. These switches occur at values of $z$ where the circle intercepts the top of the wall; for smaller $z$ values, Eq. (3-84) is used, while Eq. (3-88) gives the limit on $\phi$ for larger $z$. Furthermore, the $z$ coordinates for which the radial intersects the top of the wall have been given previously as Eq. (3-63). It can be shown that $\phi_{\min }$ changes from Eq. (3-84) to Eq. (3-88) at $z=z_{1}$, and that $\phi_{\max }$ switches form at $z=z_{2}$.

To summarize the previous discussion, in which $x_{s}^{\prime \prime}>0$ and $x_{d}^{\prime \prime}>0$, the expressions for the limits of integration on $\phi$ take on one of two different forms, according to the value of $\mathrm{z}$ and Eq. (3-63). For the class of problems in which $x_{s}^{\prime \prime}>0$ and $x_{d}^{\prime \prime}>0$,

$$
\begin{gathered}
\phi_{\min }=\left\{\begin{array}{l}
\arcsin [(\mathscr{C} \mathscr{D}) / \mathscr{C}], \mathrm{z} \leq \mathrm{z}_{1} \\
\arcsin \left[(\mathscr{D}-\mathscr{C}) / \mathscr{G}, \mathrm{z} \geq \mathrm{z}_{1}\right.
\end{array}\right. \\
\phi_{\max }=\left\{\begin{array}{l}
\arcsin [(\mathscr{C}+\mathscr{D}) / \mathscr{G}], \mathrm{z} \leq \mathrm{z}_{2} \\
\arcsin \left[(\mathscr{D}+\mathscr{E}) / \mathscr{G}, \mathrm{z} \geq \mathrm{z}_{2} .\right.
\end{array}\right.
\end{gathered}
$$

The limits above are displayed versus $z$ in Fig. 3-28 for three different source-detector geometries of this type. The angle $\xi$ is different for each case, but short computations will show that $z_{1}$ always marks the $z$ coordinate at which $\phi_{\min }$ changes formulas, and $z_{2}$ always acts as the point of change for $\phi_{\max }$. Note that the limits on $\phi$ are parabolas when plotted versus $z$; expressed another way, the intersection of a cylinder of radius $r$ and a half-plane bordering the scattering zone forms a parabola on the curved surface of the cylinder. The intersections of the two parabolas formed by the two 


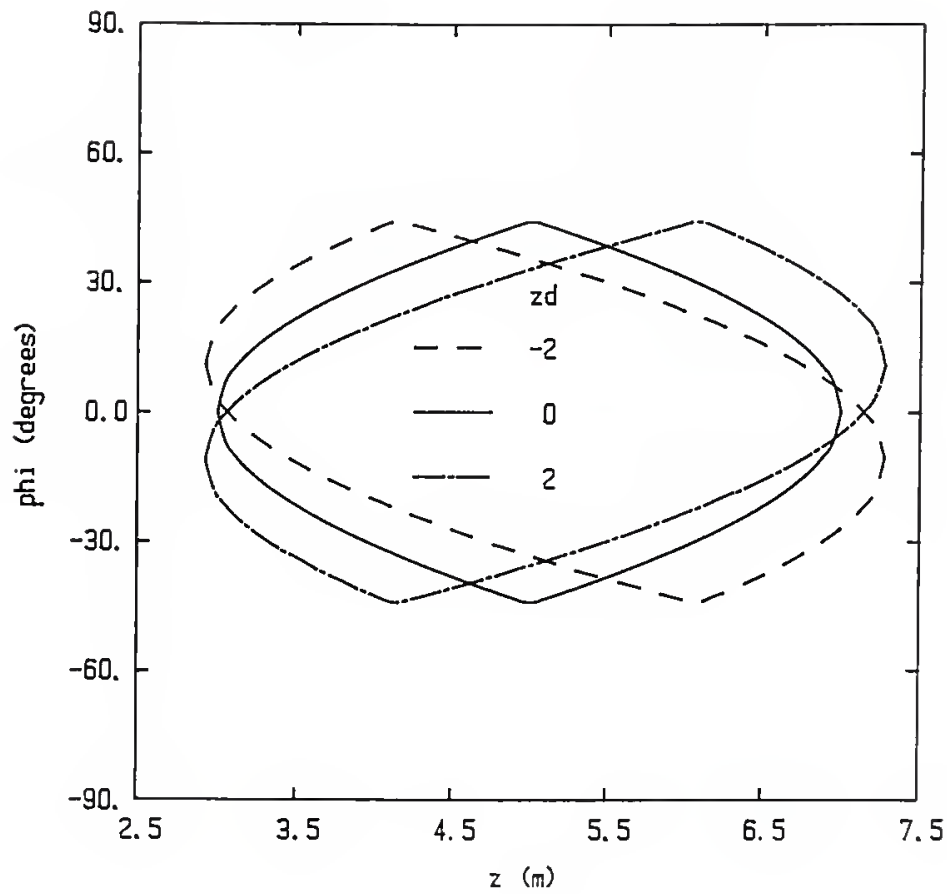

Fig. 3-28. Limits of integration over $\phi$ for source-detector geometries in which the wall extends above neither source nor detector. In all cases, $x_{s}^{\prime \prime}$ and $x_{d}^{\prime \prime}$ are positive; $x_{s}=x_{d}=y_{s}=y_{d}=5 \mathrm{~m}, r=7 \mathrm{~m}$. Note the parabolic shape of the curves. 
planes lie at $z_{1}$ and $z_{2}$, and the area enclosed by the parabolas is the region of integration over $z$ and $\phi$.

The second and third types of source-detector geometries may be solved with the knowledge obtained above. Figures 3-26(b) and (c) show how four values of $\phi$ may be required to describe the scattering zone for constant $\mathrm{r}$ and z. The cylinders of radius $r$ sketched in Fig. 3-20 still form parabolas with the half-planes bordering the scattering zone, but both parabolas open in the same direction. As before, the area enclosed by the parabolas acts as the region of integration, and the limits are described by Eqs. (3-84) through (3-91). (In the case of $x_{s}^{\prime \prime}=0$ or $x_{d}^{\prime \prime}=0$, when the top edge of the wall lies directly over the point source or point detector, respectively, the cylinder and one of the half-planes intersect to form a line instead of a parabola. The region of integration is still the area between the intersecting curves.)

Figures 3-29 and 3-30 are plots of the intersection of a cylinder with two half-planes for $x_{S}^{\prime \prime}<0$. In Fig. 3-29, the larger parabola on the left is the intersection with the lower half-plane, such as in Fig. 3-20(b). The parabola formed by the upper surface nests within it, and splits the valid values of $\phi$ into two regions past its apex in the positive $z$ direction. In the situation which created Fig. 3-30, the source-detector axis is strongly skewed, i.e., the offset of the detector from the source is relatively large. The parabola on the upper half-plane is skewed as a result; its apex does not lie between the arms of the lower parabola, thus the apex is not shown in the figure. Still, the area between the two curves is the region of integration over $z$ and $\phi$. To find the limits on $\phi$, then, the values of $z$ at which the curves intersect are needed, and the $z$ and $\phi$ coordinates of the apex of the inner 


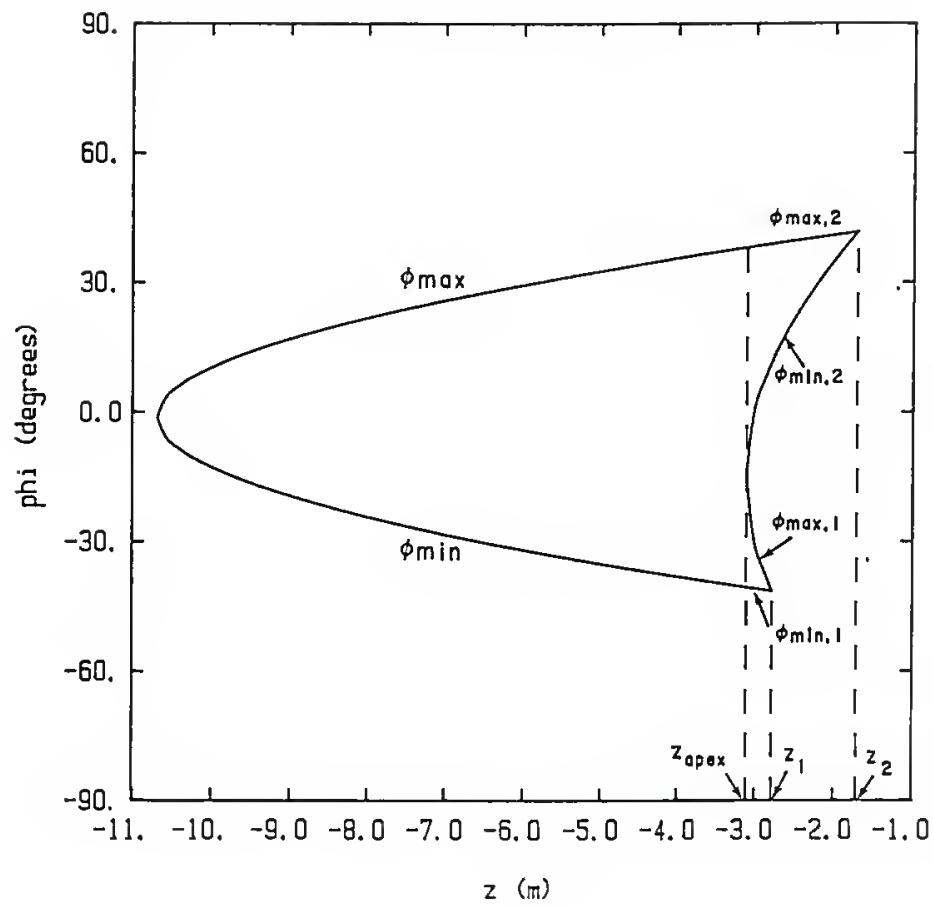

Fig. 3-29. Limits of integration over $\phi$ in a source-detector geometry for which the wall extends over the source and the $\phi$ integral breaks into two integrals over separate regions. In the case shown, $\mathrm{x}_{\mathrm{s}}=\mathrm{x}_{\mathrm{d}}=\mathrm{y}_{\mathrm{s}}=5 \mathrm{~m}, \mathrm{y}_{\mathrm{d}}$ $=25 \mathrm{~m}, z_{d}=2 \mathrm{~m}$, and $r=9 \mathrm{~m}$. 


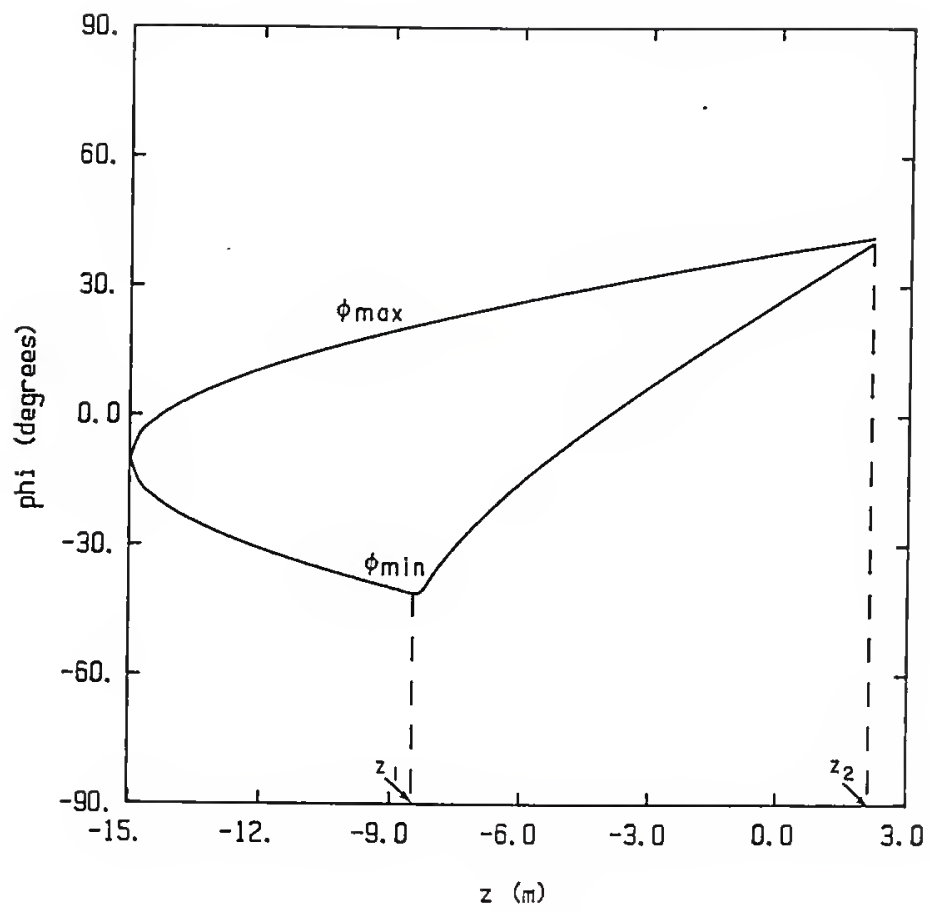

Fig. 3-30. Limits of integration over $\phi$ in a source-detector geometry for which the wall extends over the source, but the $\phi$ integral does not split into two separate regions. In the case shown, $x_{s}=x_{d}=y_{s}=5 \mathrm{~m}, y_{d}=25 \mathrm{~m}$,
$z_{d}=20 \mathrm{~m}$, and $\mathrm{I}=9 \mathrm{~m}$. 
parabola are required. The same conclusion can be drawn from the case of $x_{d}^{\prime \prime}$ $<0$ by analogous arguments.

Many of the expressions necessary have already been derived with the case of $x_{\mathrm{S}}^{\prime \prime}>0, x_{\mathrm{d}}^{\prime \prime}>0$. Figure $3-31$ is a skeletal geometry used in finding the $z$ and $\phi$ coordinates of the apex on the upper half-plane. An imaginary circle of radius $r$ about the axis in the IJKL plane would contact the half-plane in one point; therefore the radial $\mathrm{r}$ is normal to this half-plane, defined by the point detector and top edge of the wall $\left(x_{d}^{\prime \prime}<0\right)$. Since the radial is perpendicular to the surface, one would expect the result to be similar to the analysis in Fig. 3-21, the result of which is Eq. (3-57). Indeed, the only difference is a change of sign:

$$
z_{\text {apex }}=d+\frac{r\left[\left(x_{d}^{\prime}\right)^{2}+h^{2} \sin ^{2} \xi\right]^{\frac{1}{2}}}{h \cos \xi}, x_{d}^{\prime \prime}<0 .
$$

The azimuthal angle is still given by Eqs. (3-84) - (3-87); because the two values of $\phi$ converge, we have

$$
\sin \phi_{\text {apex }}=\left.(\mathscr{6} / \mathscr{C})\right|_{\mathrm{z}}=z_{\text {apex }} \quad, \quad x_{\mathrm{d}}^{\prime \prime}<0 .
$$

The results for the case of $x_{S}^{\prime \prime}<0$ should now be obvious, and so are presented without proof.

$$
z_{\text {apex }}=-\frac{\left.r\left[\left(x_{S}^{\prime}\right)\right)^{2}+h^{2} \sin ^{2} \xi\right]^{\frac{1}{2}}}{h \cos \xi}, x_{S}^{\prime \prime}<0 .
$$



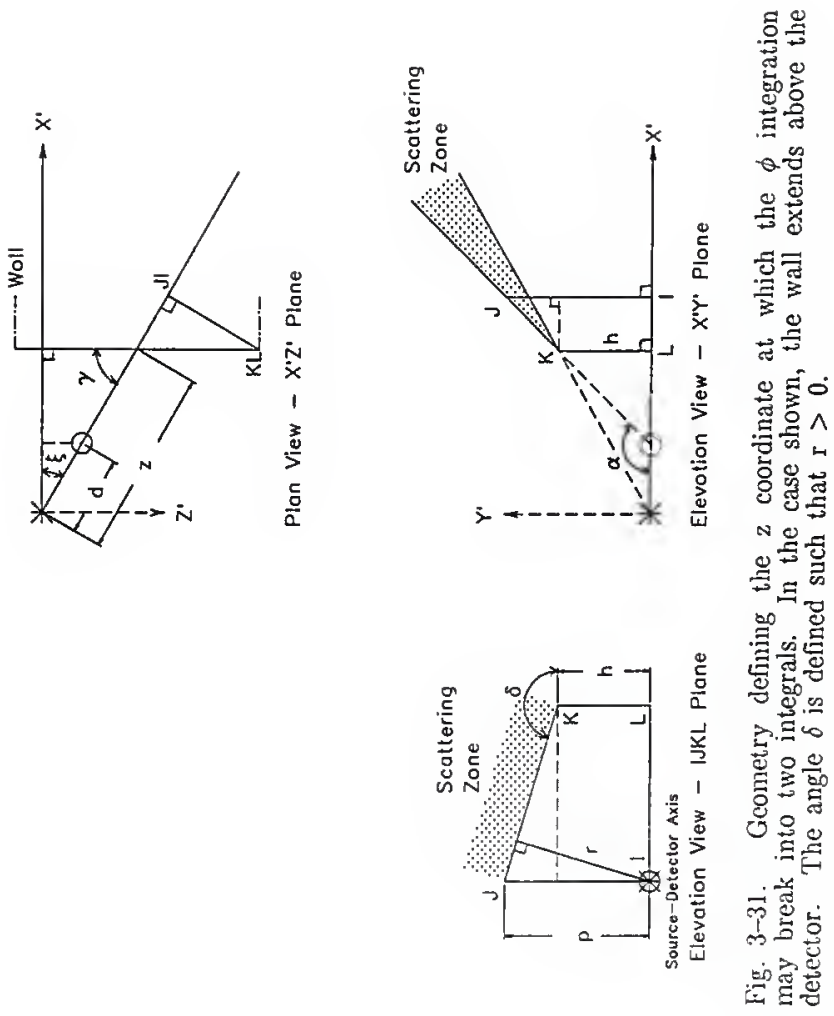


$$
\sin \phi_{\text {apex }}=\left.(\mathscr{D} / \mathscr{I})\right|_{\mathrm{z}}=z_{\text {apex }} \quad, \quad \mathrm{x}_{\mathrm{S}}^{\prime \prime}<0,
$$

where $\mathscr{D}$ and $\mathscr{F}$ are given by Eqs. (3-89) and (3-91), respectively.

These quantities are used in comparisons to determine if the azimuthal integral over $\phi$ will break into two regions, and if so, over what region of $z$ it does so. Two criteria may be deduced from Figs. 3-29 and 3-30.

(1) The $z$ coordinate locating the apex of the inner parabola, $z_{a p e x}$, must lie between the limits of integration for $z$.

(2) The $\phi$ coordinate $\phi_{\text {apex }}$ of this point must lie between the $\phi$ values of the outer parabola at $z_{a p e x}$.

If both conditions are met, the azimuthal integral will split into two regions; if not, the integral remains one.

The values of $z_{1}$ and $z_{2}$ perform a slightly different function than for the first class of geometries, where $x_{s}^{\prime \prime}>0, x_{d}^{\prime \prime}>0$. As before, they mark endpoints of the region of $z$ where Eq. (3-84) or Eq. (3-88) is valid. When $x_{\mathrm{S}}^{\prime \prime}<0$, however, and the phi integral splits into two regions, both formulas may only be used for values of $z$ smaller than $z_{1}$ or $z_{2}$; the half-planes do not exist above these values of $\mathrm{z}$ within a distance $\mathrm{r}$ of the source-detector axis. In Fig. 3-29, for example, the negative values of $\phi$ which mark the integration limits converge at $z=z_{1}$ and do not exist for higher values of $z$. The two upper values of $\phi$ are only useful for limits of integration when $z_{\text {apex }}<z \leq z_{2}$. One would correctly anticipate the figure to be reversed if $x_{d}^{\prime \prime}$ $<0$ and the phi integral splits. Then, the two lower values of $\phi$ can be used as limits if $z_{1} \leq z<z_{a p e x}$, and higher azimuthal angles may be used if $z_{2} \leq z$ $<z_{\text {apex }}$. In the earlier discussions, the two equations for $\phi_{\min }$ were used as limits only on opposite sides of $z_{1}$, and so on. 
If the phi integral does not break apart, as in Fig. 3-30, the values of $z_{1}$ and $z_{2}$ have mixed roles. In the figure, for example, $x_{s}^{\prime \prime}<0$; $z_{1}$ marks the point at which the lower limit of $\phi$ switches formulas. The lower azimuthal angle found by Eq. (3-84) gives way to the higher angle from Eq. (3-88) as z increases. The parabola created by the intersection of the cylindrical surface and the upper half-plane [for example, see Fig. 3-20(b)] has its apex well outside the region of integration, and consequently only one leg of that parabola is used for limits on $\phi$. This leg defines $\phi_{\min }$ for $z_{1} \leq z \leq z_{2}$. The offset $z_{d}$ of the detector is positive in the geometry which produced the figure; if $z_{\mathrm{d}}$ were -20 instead of +20 , the opposite leg of the upper parabola would be used alone to define $\phi_{\max }$ over part of the range of $z$. The analogies to the case of $x_{d}^{\prime \prime}<0$ should be clear; Eqs. (3-84) and (3-88) map parabolas open to the left, and only one leg of Eq. (3-84) is used over the range of $z$. (Each plot of $\phi$ versus $z$ is for constant $r$.)

This discussion of the phi limits for cases when the scattering zone is not between the source and detector is presented in an organized fashion in Table 3-5. The examples have concerned source-detector arrangements in which $x_{s}^{\prime \prime}<0$; analogies to $x_{d}^{\prime \prime}<0$ are easily made, and may be understood more easily with the help of the table. The formulas for $\phi, z_{\text {apex }}$ and $\phi_{\text {apex }}$ are repeated here in the notation of the table.

$$
\begin{aligned}
& \sin \phi_{1}=(\mathscr{6}-\mathscr{D}) / \mathscr{C} \\
& \sin \phi_{2}=(\mathscr{6}+\mathscr{D}) / \mathscr{C} \\
& \sin \phi_{3}=(\mathscr{D}-\mathscr{C}) / \mathscr{F}
\end{aligned}
$$




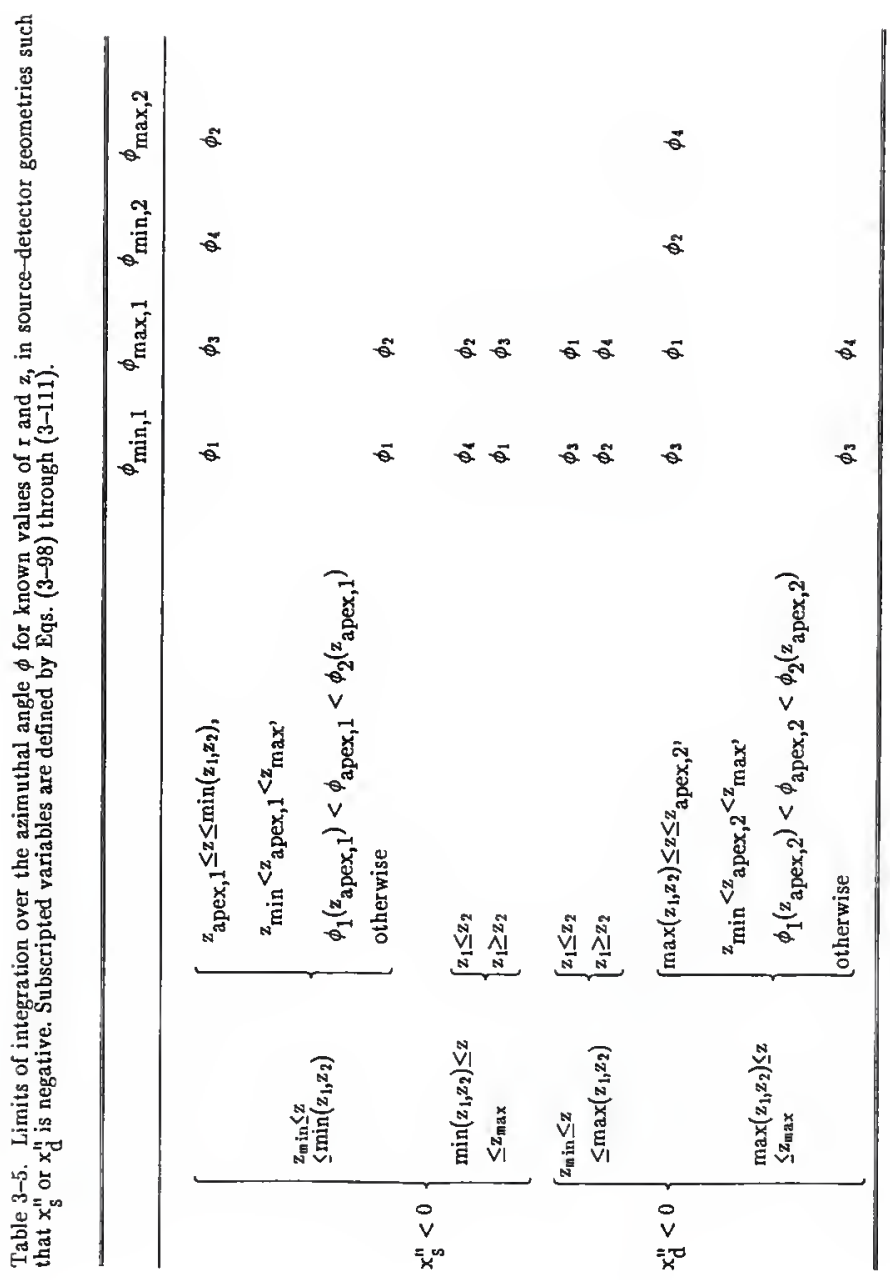




$$
\sin \phi_{4}=(\mathscr{D}+\mathscr{E}) / \mathscr{F}
$$

where

$$
\begin{aligned}
& \mathscr{b}=-\mathrm{h}^{2} \sin \xi(\mathrm{d}-\mathrm{z}) \cos \xi, \\
& \mathscr{B}=\left\{\left(\mathrm{rx} \mathrm{d}_{\mathrm{d}}^{\prime}\right)^{2}\left[\left(\mathrm{x}_{\mathrm{d}}^{\prime}\right)^{2}+(\mathrm{h} \sin \xi)^{2}\right]-\left[\mathrm{h} \mathrm{x}_{\mathrm{d}}^{\prime}(\mathrm{d}-\mathrm{z}) \cos \xi\right]^{2}\right\}^{\frac{1}{2}}, \\
& \mathscr{E}=\mathrm{r}\left[\left(\mathrm{x}_{\mathrm{d}}^{\prime}\right)^{2}+(\mathrm{h} \sin \xi)^{2}\right] \text {, } \\
& \mathscr{D}=\mathrm{h}^{2} \mathrm{z} \cos \xi \sin \xi, \\
& \mathscr{E}=\left\{\left(\mathrm{rx}_{\mathrm{S}}^{\prime}\right)^{2}\left[\left(\mathrm{x}_{\mathrm{S}}^{\prime}\right)^{2}+(\mathrm{h} \sin \xi)^{2}\right\}-\left[\mathrm{hzx_{ \textrm {S } } ^ { \prime }} \cos \xi\right]^{2}\right\}^{\frac{1}{2}},
\end{aligned}
$$

and

$$
\begin{aligned}
& \mathscr{F}=\mathrm{r}\left[\left(\mathrm{x}_{\mathrm{s}}^{\prime}\right)^{2}+(\mathrm{h} \sin \xi)^{2}\right] \\
& \mathrm{z}_{\text {apex }, 1}=-\frac{\mathrm{r}\left[\left(\mathrm{x}_{\mathrm{s}}^{\prime}\right)^{2}+\mathrm{h}^{2} \sin ^{2} \xi\right]^{\frac{1}{2}}}{\mathrm{~h} \cos \xi}, \quad \mathrm{x}_{\mathrm{s}}^{\prime \prime}<0 \\
& \mathrm{z}_{\text {apex }, 2}=\mathrm{d}+\frac{\mathrm{r}\left[\left(\mathrm{x}_{\mathrm{d}}^{\prime}\right)^{2}+\mathrm{h}^{2} \sin ^{2} \xi\right]^{\frac{1}{2}}}{\mathrm{~h} \cos \xi}, \quad \mathrm{x}_{\mathrm{d}}^{\prime \prime}<0 \\
& \sin \phi_{\text {apex }, 1}=\mathscr{D} /\left.\mathscr{S}\right|_{\mathrm{z}}=\mathrm{z}_{\text {apex }, 1}, \\
& \sin \phi_{\text {apex }, 2}=\mathscr{L} /\left.\mathscr{C}\right|_{\mathrm{z}}=\mathrm{z}_{\text {apex }, 2}<0
\end{aligned}
$$

In summary, Table $3-6$ lists limits of integration over the azimuthal angle $\phi$ for all possible source-detector geometries and known values of $\mathrm{r}$ and z. The notation is the same as that of Table 3-5. It is assumed that the limits are not evaluated at $r=r_{\min }$, which is a trivial case; otherwise, the limits hold for all $I$ and $z$. 


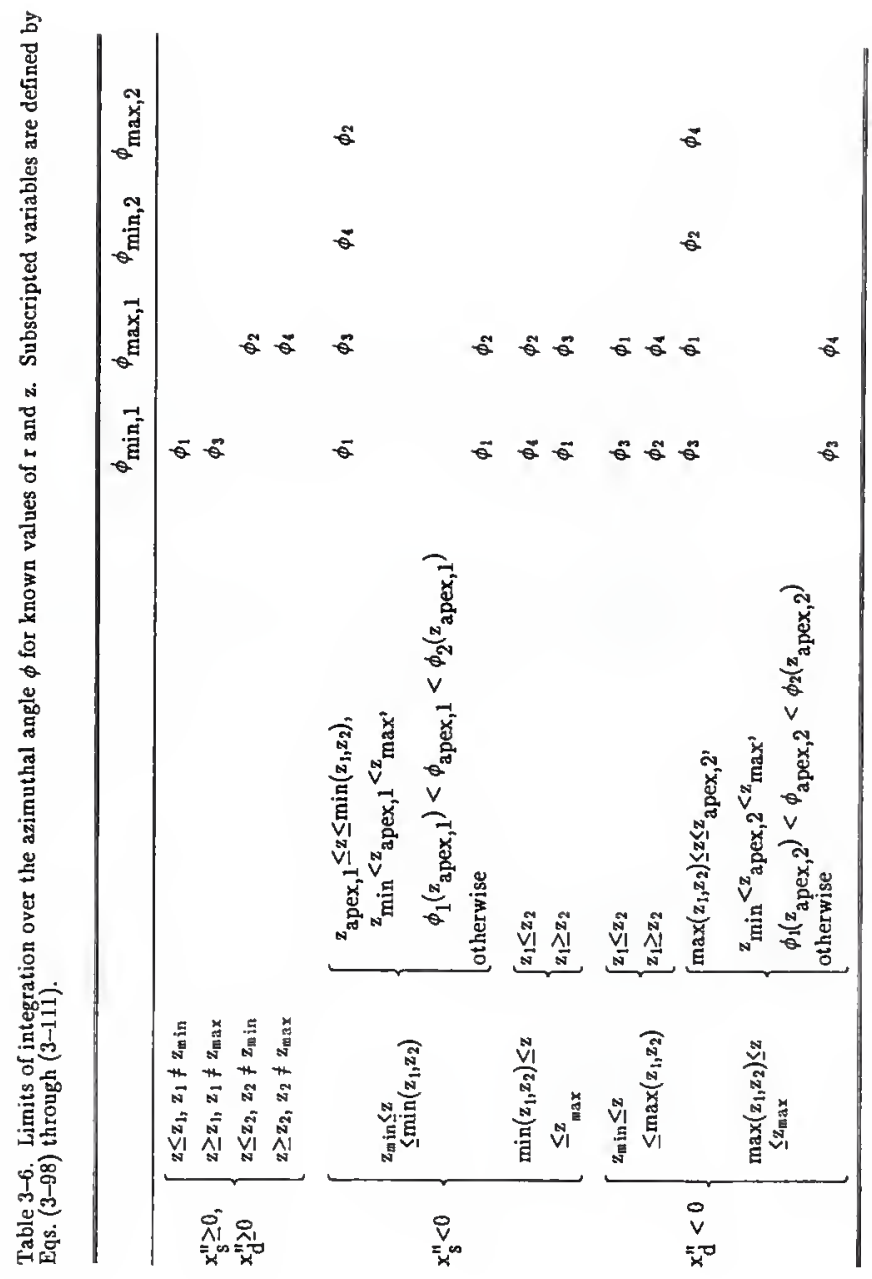


The patterns inherent in the integration limits may be seen in Figs. 3-32 through 3-34. In each graph, limits of integration on $\phi$ are plotted versus $z$ for a given source-detector geometry and increasing values of $r$. The graphs are, in fact, contour plots of the intersection of a cylindrical surface of radius $r$ with the boundaries of the scattering zone. Given the radius $r$, the differential scattering volume for a gamma-ray must lie on the surface of the appropriate cylinder; the contour plots describe the section of each cylinder within the scattering zone, thus the area of integration. Fig. 3-20 illustrates this concept well.

\subsubsection{Numerical Evaluation}

The semi-infinite wall skyshine problem discussed in this work is solved numerically by the computer code WALLGP, listed in Appendix B. This FORTRAN program calculates the exposure rate, kerma rate or dose equivalent due to an isotropic point source emitting one photon per second. The locations of the point source and point detector with respect to the wall are specified in the input; if a concrete shield is involved, its thickness and density are also read by the input routine. Results are presented as response per photon, and also as response per photon multiplied by the square of the source-detector distance. The second quantity is sometimes preferred for comparisons, since it varies less with distance. Because the interaction coefficients of Hubbell (Hu82), the geometric-progression buildup factor formula (Ha86, RS86), and the adaptive Gaussian quadrature all proved successful in the SILOGP code for the silo problem, all these features were employed in WALLGP. Some approximations made by Roseberry (Ros0) were also tested 


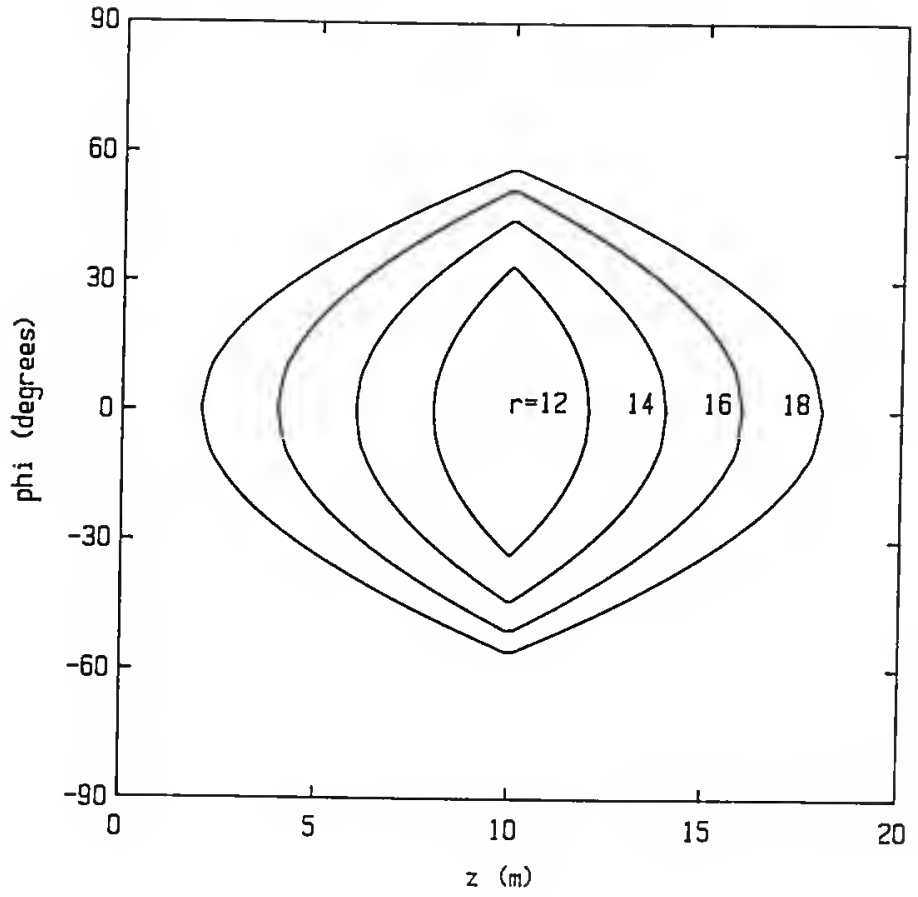

Fig. 3-32. Contour plot of the limits of integration in the wall geometry for increasing values of the radial coordinate $r$. The source and detector lie in a plane normal to the wall; $x_{s}=x_{d}=y_{s}=y_{d}=10 \mathrm{~m}$. 


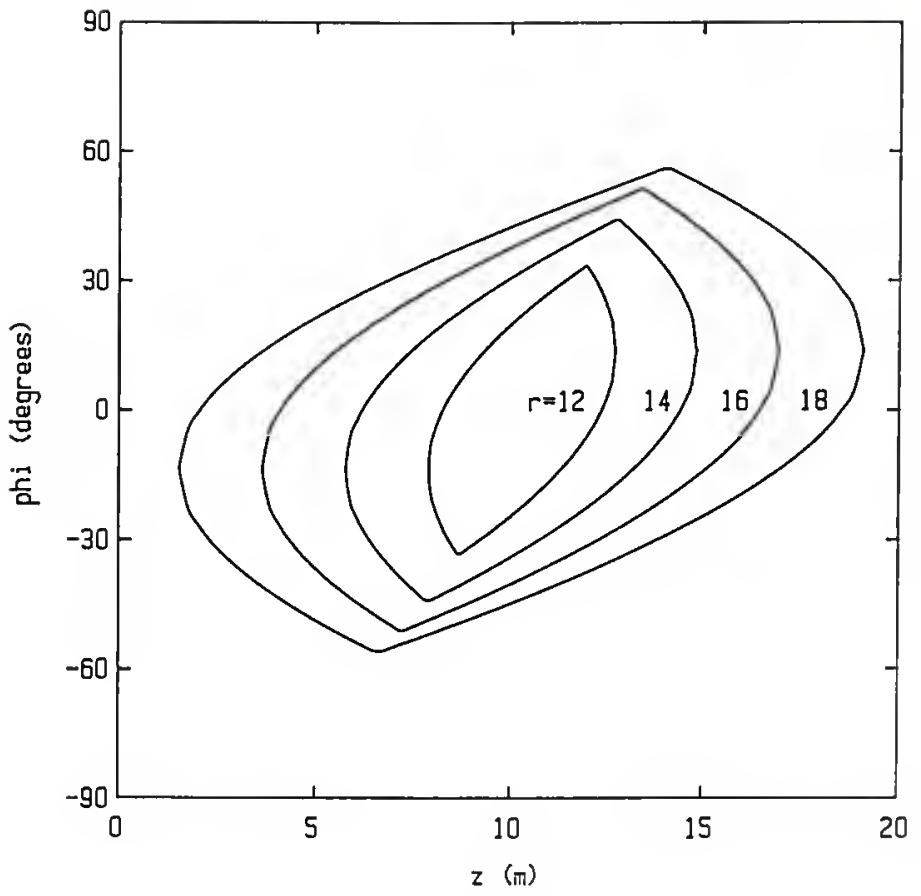

Fig. 3-33. Contour plot of the limits of integration in the wall geometry for increasing values of the radial coordinate $r$. The source and detector are offset by $z_{d}=5 \mathrm{~m}$; the wall extends over neither source nor detector. $x_{s}=x_{d}=$ $\mathrm{y}_{\mathrm{s}}=\mathrm{y}_{\mathrm{d}}=10 \mathrm{~m}$. 


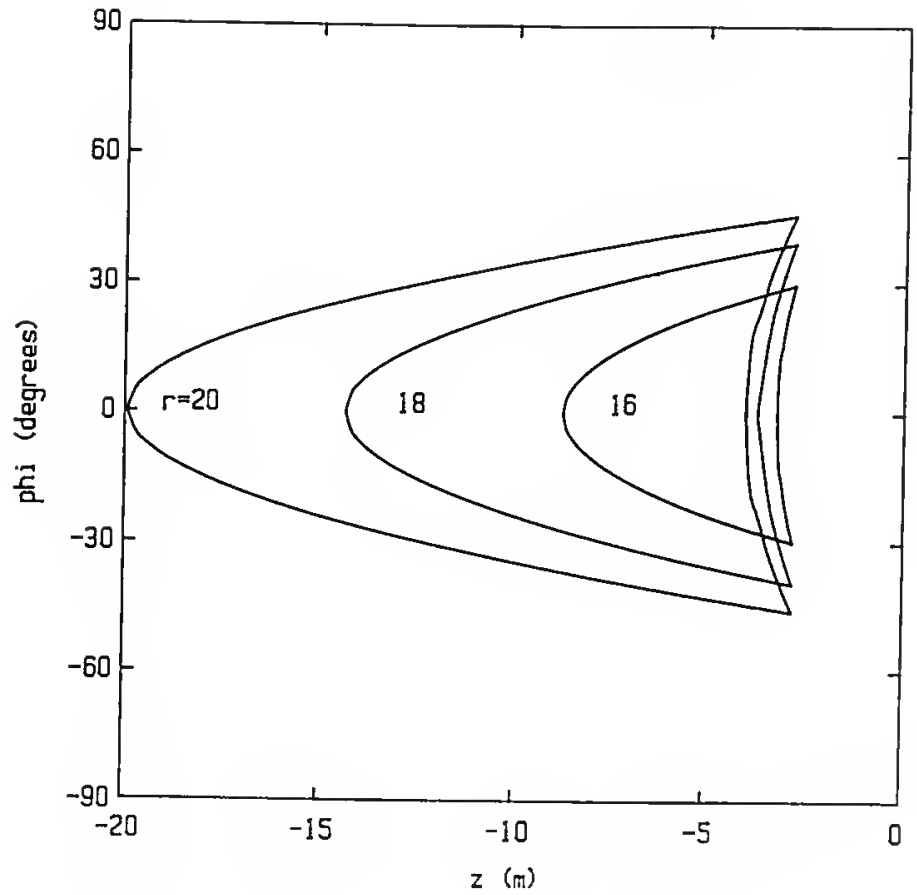

Fig. 3-34. Contour plot of the limits of integration in the wall geometry for increasing values of the radial coordinate $r$. The source and detector lie in a plane normal to the wall, and the wall extends over the source in the cylindrical frame of reference. $x_{s}=x_{d}=y_{s}=10 \mathrm{~m} ; y_{d}=40 \mathrm{~m} ; z_{d}=0$. 
in WALLGP, along with approximations unique to the wall geometry. Their usefulness is discussed as the final topic of this chapter.

In all calculations performed by the code WALLGP, air density is taken to be constant throughout the system. This is assumed in many computer codes, and since most physical problems considered in this work have less than $2000 \mathrm{~m}$ separating the source and detector, the assumption seems valid. Uniform density of the concrete shielding is also assumed for simplicity.

For a variety of source energies and source-detector arrangements, detector responses were computed using double precision and single precision versions of WALLGP. To three significant digits, no differences were found between results of the two programs. Although single-precision computations require shorter computing times and fewer resources, double precision results are more accurate in most applications. It was decided to employ double precision in the final version of WALLGP, for the advantage of accuracy.

One assumption made by Roseberry to simplify the computer code SKY was not made in the code for the wall skyshine geometry. Roseberry approximated the path length a traveled by source photons in air as the total path length $\bar{a}$ from the point source to the scattering volume $\mathrm{dV}$. In the absence of a concrete shield over the source, $\mathrm{a}=\overline{\mathrm{a}}$, and the assumption is correct. If a concrete shield is present, the assumption can introduce error, especially in instances when the differential scattering volume lies close to the source. The effect is to lower the computed detector response by artificially reducing the uncollided flux entering $\mathrm{dV}$. Since Roseberry's code included the assumption, yet overestimated the results of a benchmark experiment (Ros0), the approximation was justified in SIY. 
In this work, a more rigorous approach has been taken. In the absence of a concrete shield, the distance a along the first leg of the gamma-ray path is independent of the azimuthal angle $\phi$ (analogous to the angle $\epsilon$ in the silo geometry). Further, every integrand of Eq. (3-47) is independent of the azimuthal angle, and the innermost integral over $d \phi$ equals the integrand times the difference in limits. If a shield is present, $a$ is dependent on $\phi$ through Eqs. (3-32) and (3-46), since the distance $a_{c}$ through the roof is nonzero. In the code WALLGP, Eq. (3-32) is used as written if the geometry involves a shield; thus, the approximation of Roseberry is not used. If no shield is involved, WALLGP computes a as part of the $z$ integrand and uses Eq. (3-32) with $\mathrm{a}_{\mathrm{c}}=0$, as Roseberry did for all cases.

An important input parameter to the wall program is the photon path length cutoff value in mean free paths, variable CUTMFP. Gamma rays which must travel farther than the cutoff value to reach the detector are ignored in computation of response. Also, the cutoff value is used to compute limits on the integration variables $\mathbf{r}$ and $z$ via Eqs. (3-51), (3-68) and (3-69). Roseberry (Ro80) chose ten mean free paths in air and fifteen $\mathrm{mfp}$ in concrete as cutoff values. In WALLGP, a single value is compared against the mean free paths in air and concrete traversed by a photon, avoiding double standards.

To find a suitable value of the cutoff, exposure rates were computed for fourteen different source-detector arrangements, listed in Table $3-7$. The test cases were selected from reference problems described in Chapter 4; exposure rates were found using cutoff values of 10,20,30, and 40 mean free paths. The results, presented in Table $3-8$, point toward a cutoff of 40 mean free 
Table 3-7. Test cases used to investigate the convergence of the computer code WALLGP. The test cases are selected from reference problems presented in Chapter 4 , and are grouped according to the parameter which varies between cases.

\begin{tabular}{|c|c|c|c|c|c|c|c|c|c|}
\hline $\begin{array}{l}\text { Test } \\
\text { case }\end{array}$ & $\begin{array}{l}\text { Source } \\
\text { energy } \\
(\mathrm{NeV})\end{array}$ & $\begin{array}{c}x_{s} \\
(m)\end{array}$ & $\begin{array}{l}\mathrm{x}_{\mathrm{d}} \\
(\mathrm{m})\end{array}$ & $\begin{array}{l}y_{s} \\
(m)\end{array}$ & $\stackrel{y_{d}}{(m)}$ & $\begin{array}{l}z_{d} \\
(m)\end{array}$ & $\begin{array}{l}t \\
(m)\end{array}$ & $\begin{array}{l}\rho_{\mathrm{conc}} \\
\left(\mathrm{g} / \mathrm{cm}^{3}\right)\end{array}$ & $\begin{array}{c}\rho_{\mathrm{air}} \\
\left(\mathrm{mg} / \mathrm{cm}^{3}\right)\end{array}$ \\
\hline $\begin{array}{l}1 \\
2 \\
3\end{array}$ & $\begin{array}{l}6.2 \\
6.2 \\
6.2\end{array}$ & $\begin{array}{r}40 \\
400 \\
750\end{array}$ & $\begin{array}{r}40 \\
400 \\
750\end{array}$ & $\begin{array}{l}0.00001 \\
0.00001 \\
0.00001\end{array}$ & $\begin{array}{l}0.00001 \\
0.00001 \\
0.00001\end{array}$ & $\begin{array}{l}0 \\
0 \\
0\end{array}$ & $\begin{array}{l}0 \\
0 \\
0\end{array}$ & & $\begin{array}{l}1.22 \\
1.22 \\
1.22\end{array}$ \\
\hline $\begin{array}{l}4 \\
5 \\
6 \\
7\end{array}$ & $\begin{array}{l}6.13 \\
6.13 \\
6.13 \\
6.13\end{array}$ & $\begin{array}{l}1 \\
1 \\
1 \\
1\end{array}$ & $\begin{array}{r}9 \\
799 \\
9 \\
799\end{array}$ & $\begin{array}{l}0.7 \\
0.7 \\
0.7 \\
0.7\end{array}$ & $\begin{array}{l}0.7 \\
0.7 \\
0.7 \\
0.7\end{array}$ & $\begin{array}{l}0 \\
0 \\
0 \\
0\end{array}$ & $\begin{array}{l}0 \\
0 \\
0.3 \\
0.3\end{array}$ & $\begin{array}{l}2.13 \\
2.13\end{array}$ & $\begin{array}{l}1.25 \\
1.25 \\
1.25 \\
1.25\end{array}$ \\
\hline $\begin{array}{r}8 \\
9 \\
10\end{array}$ & $\begin{array}{c}0.1 \\
1.0 \\
10\end{array}$ & $\begin{array}{l}500 \\
500 \\
500\end{array}$ & $\begin{array}{l}500 \\
500 \\
500\end{array}$ & $\begin{array}{l}0.00001 \\
0.00001 \\
0.00001\end{array}$ & $\begin{array}{l}0.00001 \\
0.00001 \\
0.00001\end{array}$ & $\begin{array}{l}0 \\
0 \\
0\end{array}$ & $\begin{array}{l}0 \\
0 \\
0\end{array}$ & & $\begin{array}{l}1.25 \\
1.25 \\
1.25\end{array}$ \\
\hline $\begin{array}{l}11 \\
12\end{array}$ & $\begin{array}{l}1.25 \\
1.25\end{array}$ & $\begin{array}{l}3 \\
3\end{array}$ & $\begin{array}{l}100 \\
100\end{array}$ & $\begin{array}{l}3.0 \\
3.0\end{array}$ & $\begin{array}{l}3.0 \\
3.0\end{array}$ & $\begin{array}{r}10 \\
300\end{array}$ & $\begin{array}{l}0.1 \\
0.1\end{array}$ & $\begin{array}{l}2.35 \\
2.35\end{array}$ & $\begin{array}{l}1.2 \\
1.2\end{array}$ \\
\hline $\begin{array}{l}13 \\
14\end{array}$ & $\begin{array}{l}1.25 \\
1.25\end{array}$ & $\begin{array}{l}3 \\
3\end{array}$ & $\begin{array}{l}100 \\
100\end{array}$ & $\begin{array}{l}3.0 \\
3.0\end{array}$ & $\begin{array}{l}3.0 \\
3.0\end{array}$ & $\begin{array}{l}10 \\
10\end{array}$ & $\begin{array}{l}0.01 \\
1.0\end{array}$ & $\begin{array}{l}2.35 \\
2.35\end{array}$ & $\begin{array}{l}1.2 \\
1.2\end{array}$ \\
\hline
\end{tabular}


Table 3-8. Behavior of detector responses computed by WALLGP with changes in the mean free path cutoff value. The test cases are described in detail in Table $3-7$. All computations were performed using a convergence criterion of $1 \%$ difference between successive evaluations of the exposure integral.

\begin{tabular}{lllll}
\hline \hline \multicolumn{5}{c}{$\begin{array}{c}\text { Normal ized exposure } \\
\text { (R/photon) }\end{array}$} \\
\cline { 2 - 5 } Test case & $\begin{array}{l}10 \mathrm{mfp} \\
\text { cutoff }\end{array}$ & $\begin{array}{l}20 \mathrm{mfp} \\
\text { cutoff }\end{array}$ & $\begin{array}{l}30 \mathrm{mf} p \\
\text { cutoff }\end{array}$ & $\begin{array}{l}40 \mathrm{mfp} \\
\text { cutof } \mathrm{f}\end{array}$ \\
\hline & & & & \\
1 & $1.003(-19)^{*}$ & $1.003(-19)$ & $9.004(-20)$ & $6.512(-20)$ \\
2 & $1.134(-21)$ & $1.134(-21)$ & $1.134(-21)$ & $1.134(-21)$ \\
3 & $7.113(-23)$ & $7.124(-23)$ & $7.806(-23)$ & $7.118(-23)$ \\
4 & $8.323(-20)$ & $8.323(-20)$ & $8.168(-20)$ & $8.323(-20)$ \\
5 & $1.050(-23)$ & $1.084(-23)$ & $1.084(-23)$ & $1.085(-23)$ \\
6 & $1.358(-20)$ & $1.358(-20)$ & $1.342(-20)$ & $1.358(-20)$ \\
7 & $1.209(-24)$ & $1.657(-24)$ & $1.662(-24)$ & $1.657(-24)$ \\
8 & $0 \mathrm{a}$ & $8.571(-28)$ & $1.377(-27)$ & $1.382(-27)$ \\
9 & $1.274(-23)$ & $1.399(-23)$ & $1.400(-23)$ & $1.400(-23)$ \\
10 & $6.724(-22)$ & $6.708(-22)$ & $6.792(-22)$ & $6.710(-22)$ \\
11 & $3.826(-21)$ & $3.827(-21)$ & $3.827(-21)$ & $3.827(-21)$ \\
12 & $4.678(-22)$ & $4.719(-22)$ & $4.720(-22)$ & $4.718(-22)$ \\
13 & $8.992(-21)$ & $9.071(-21)$ & $9.135(-21)$ & $9.173(-21)$ \\
14 & $0 \mathrm{~b}$ & $4.107(-26)$ & $4.168(-26)$ & $4.162(-26)$ \\
& & & & \\
\hline \hline
\end{tabular}

*1.003(-19) $=1.003 \times 10^{-19}$

aSource-detector distance in mean free paths is larger than cutoff value; WALL performs no calculations.

bConcrete shield thickness in mean free paths is larger than cutoff value; HALL performs no calculations. 
paths as the optimum value. Note that a cutoff of $10 \mathrm{mfp}$ with $100 \mathrm{~cm}$ of concrete overhead eliminates all contributions to detector response; one meter of concrete corresponds to over 13 mean free paths for a $1.25 \mathrm{MeV}$ photon. Similarly, if the source-detector distance equals $1000 \mathrm{~m}$, it becomes physically impossible for a $0.1 \mathrm{MeV}$ gamma ray to travel only $10 \mathrm{mfp}$ and reach the detector; $1000 \mathrm{~m}$ in air corresponds roughly to $18.5 \mathrm{mfp}$ for these photons. Larger cutoff values will avoid this problem and allow computations for larger source-detector distances. On the other hand, the geometric progression buildup factors used in the code only extend to 40 mean free paths (RSs6), so longer distances must be eliminated from consideration. The criterion of 40 mfp keeps as many dose contributions as possible in computations, and holds the computations to conditions for which the buildup data are valid.

The first test case, however, shows that CUTMFP is not the only variable important to convergence; conputed exposure actually decreases in this case as CUTMFP increases. Another cause for concern is the Gauss quadrature used to integrate Eq. (3-47). Gauss quadrature is normally not recommended for integration of ill-behaved functions or functions with discontinuous derivatives. An advantage of the adaptive Gaussian quadrature subroutine used in this work is its ability to work with such functions. Figures 3-32, 3-33 and 3-34 demonstrate that the limits of integration on $\phi$ are not at all smooth, so that the exposure rate integral equations for the wall geometry can be expected to "misbehave" over the range of $z$. The quadrature routine used here can account for such behavior if sufficient accuracy is requested by the calling program. Therefore, tests were run to determine a sufficient value of the user-supplied error parameter DEL. The 
value of the integral computed by the quadrature routine will have a maximum error of DEL times the true value of the integral being evaluated.

Table 3-9 presents exposure values computed by WALLGP for most of the geometries presented in Table 3-7, with DEL ranging from 0.05 to 0.001 . The differences in detector responses computed with error criteria of $\mathrm{DEL}=0.01$ and $\mathrm{DEL}=0.001$ are nearly always a fraction of one percent. The differences are larger for short source-detector distances, such as in test cases 1, 4 and 6. Case 1, in which the source and detector are $80 \mathrm{~m}$ apart and nearly on a line of sight, requires $0.1 \%$ error for accurate results (compare with Table 3-8). Alternately, a smaller value of MFPCUT in such instances may help, but is not recommended.

Though $0.1 \%$ accuracy may be desirable, values of $\mathrm{DEL}=0.001$ can result in impractical computation times in most instances. For normal calculations, it is suggested that DEL $=0.01$ be entered as the error parameter; this will produce accurate answers in a reasonable time. For source-detector distances below $100 \mathrm{~m}$, especially with low walls, DEL $=0.001$ will be necessary.

In summary, it was assumed in the wall skyshine code that photons which travel more than forty mean free paths in air and/or concrete produce a negligible response at a point detector. Unlike Roseberry's silo method, the wall method computes the distance traveled by a source photon in air exactly. Double precision computations were found to be accurate, as was a requirement of $1 \%$ error in the integration routines. The final version of the program WALLGP is listed in Appendix $B$; results of validation tests are presented in the next chapter. 
Table 3-9. Behavior of detector responses computed by WALLGP with changes in convergence criteria. The estimated error equals the product of the convergence parameter DEL and the computed detector response; the value of DEL in each case is the percent error expressed as a decimal. The test cases are described in detail in Table 3-7. All computations were performed using a mean free path cutoff value of $40 \mathrm{mfp}$.

\begin{tabular}{|c|c|c|c|c|c|c|}
\hline \multirow[b]{2}{*}{$\begin{array}{l}\text { Test } \\
\text { case }\end{array}$} & \multicolumn{6}{|c|}{$\begin{array}{c}\text { Normalized exposure } \\
\text { (R/photon) }\end{array}$} \\
\hline & $\begin{array}{l}5 \% \\
\text { error }\end{array}$ & $\begin{array}{l}2 \% \\
\text { error }\end{array}$ & $\begin{array}{l}1 \% \\
\text { error }\end{array}$ & $\begin{array}{l}0.5 \% \\
\text { error }\end{array}$ & $\begin{array}{l}0.2 \% \\
\text { error }\end{array}$ & $\begin{array}{l}0.1 \% \\
\text { error }\end{array}$ \\
\hline $\begin{array}{l}1 \\
2 \\
3\end{array}$ & $\begin{array}{l}6.113(-20) \\
1.287 \\
7.006(-21)\end{array}$ & $\begin{array}{l}* 6.393(-20) \\
1.130(-21) \\
6.994(-23)\end{array}$ & $\begin{array}{l}6.512(-20) \\
1.134(-21) \\
7.118(-23)\end{array}$ & $\begin{array}{l}7.777(-20) \\
1.134(-21) \\
7.124(-23)\end{array}$ & $\begin{array}{l}8.806(-20) \\
1.134(-21) \\
7.121(-23)\end{array}$ & $\begin{array}{l}9.158(-20) \\
1.134(-21) \\
7.121(-23)\end{array}$ \\
\hline $\begin{array}{l}4 \\
5 \\
6 \\
7\end{array}$ & $\begin{array}{l}8.346(-20) \\
1.084(-23) \\
1.360(-20) \\
1.772(-24)\end{array}$ & $\begin{array}{l}8.346(-20) \\
1.085(-23) \\
1.358(-20) \\
1.669(-24)\end{array}$ & $\begin{array}{l}8.323(-20) \\
1.085(-23) \\
1.358(-20) \\
1.657(-24)\end{array}$ & $\begin{array}{l}8.294(-20) \\
1.083(-23) \\
1.357(-20) \\
1.657(-24)\end{array}$ & $\begin{array}{l}7.933(-20) \\
1.080(-23) \\
1.322(-20) \\
1.656(-24)\end{array}$ & $\begin{array}{l}7.867(-20) \\
1.079 \\
1.322(-23) \\
1.657(-24)\end{array}$ \\
\hline $\begin{array}{r}8 \\
9 \\
10\end{array}$ & $\begin{array}{l}1.382(-27) \\
1.319 \\
6.757(-23)\end{array}$ & $\begin{array}{l}1.382(-27) \\
1.390(-23) \\
6.729(-22)\end{array}$ & $\begin{array}{l}1.382(-27) \\
1.400(-23) \\
6.710(-22)\end{array}$ & $\begin{array}{l}1.377(-27) \\
1.399(-23) \\
6.743(-22)\end{array}$ & $\begin{array}{l}1.376(-27) \\
1.399(-23) \\
6.744(-22)\end{array}$ & $\begin{array}{l}1.376(-27) \\
1.398(-23) \\
6.726(-22)\end{array}$ \\
\hline $\begin{array}{l}11 \\
12\end{array}$ & $\begin{array}{l}3.836(-21) \\
4.709(-22)\end{array}$ & $\begin{array}{l}3.827(-21) \\
4.718(-22)\end{array}$ & $\begin{array}{l}3.827(-21) \\
4.718(-22)\end{array}$ & $\begin{array}{l}3.822(-21) \\
4.709(-22)\end{array}$ & $\begin{array}{l}3.820(-21) \\
4.709(-22)\end{array}$ & $\begin{array}{l}3.820(-21) \\
4.709(-22)\end{array}$ \\
\hline
\end{tabular}

$* 6.113(-20)=6.113 \times 10^{-20}$ 


\subsection{RESULTS AND VALIDATION OF THE CODES DEVELOPED IN THIS WORK}

A necessary step in the development of a numerical model is comparison of its results to real data from the physical system being modeled, or to results accepted as correct by authorities in the field. The numerical models for gamma-ray skyshine analysis presented in the previous chapter will now be validated by comparisons to standards and results from other computations. In the case of the silo geometry modeled by SILOGP, benchmark experimental data will be presented for comparison, but no such data are available for the wall geometry modeled by WALLGP.

The American National Standard which addresses skyshine measurements and computations includes reference calculations for this purpose. One of the reference problems involves a point isotropic source of $16 \mathrm{~N}$ gamma rays sixty feet above an air-ground interface. Detectors are placed along the ground at specific distances from the source, and dose rates are reported along the range of detectors. (AN87) While the problem does not involve either a silo or a wall shielding the detector, both SILOGP and WALLGP can model situations approaching the problem. By using an open silo with a full angle of collimation of $180^{\circ}$, or a wall of very small height between source and detector, the two codes can approximate the open point source and produce results comparable to the ANSI reference calculations. A second ANSI reference problem places the ${ }^{16} \mathrm{~N}$ source inside a rectangular concrete building without a roof. The detector response in this problem is likely to be dependent upon the solid angle into which source photons are collimated. The 
cylindrical silo of SILOGP would simulate the rectangular building better than a single wall, but would still introduce error through incorrect collimation. Therefore, only the first problem will be used for reference in this work.

A problem similar to the ANSI reference calculation was used in the validation of MicroSkyshine (Fa87,Sh87) and may also be used to advantage here. Air kerma buildup factors were determined from moments-method calculations by Chilton et al. (Ch80) These widely-accepted buildup factors were manipulated to give exposures due to a point source in infinite air emitting photons only in a hemisphere of directions; this hemisphere lies above a plane containing the source and detector. This $2 \pi$ problem is easily approximated by the MicroSkyshine code, and exposures computed by that code were compared against the results inferred from the buildup factors of Chilton et al. Both SILOGP and WALLGP can approximate the same geometry, and this problem will be solved with both codes as another test of their accuracy.

Experimental data reported by Nason et al. (Na81) and Roseberry (Ros0) from the KSU benchmark skyshine experiment will be compared to results generated by SILOGP for the geometry and conditions of the experiment. Roseberry cited results of the transport code DOT, which others prepared for the study; since this code is in use today, those computed exposure rates will also be compared to SILOGP values. Finally, WALLGP and SILOGP will be tested extensively against the MicroSkyshine program for microcomputers, in an attempt to validate all three codes. The MicroSkyshine tests will include variations of geometry parameters as well as benchmark and reference problems. 


\subsection{Comparison of SILOGP to Other Methods and Benchmark Data}

The code SILOGP, which solves the problem of a point source inside a collimating silo, will be tested first. Data from seven sources were selected to validate SILOGP; four problem geometries were studied, and observations were made of changes in detector response with chosen parameters. One of the four systems is that of the benchmark skyshine experiments reported by Nason et al., for which measured responses and responses computed by the code DOT 3.5 are available (Ro80,Na81). A similar configuration was used in the validation of MicroSkyshine, one that is identical to the experimental conditions except for changes in material properties (Fa87, Sh87). The third problem is a standard from ANSI/ANS-6.6.1-1987, Reference Problem I.1. (AN87) The fourth problem, also presented in references on the MicroSkyshine code, is the $2 \pi$ problem for which buildup factors of Chilton et al. (Ch80) were used to predict exposure rates. The last two problems do not involve silos or shielding, but are useful in testing the behavior of SILOGP in the limiting case of $2 \pi$ geometry.

\subsubsection{Comparisons to Benchmark Study}

The skyshine benchmark experiment (Na81) described in Chapter 2 provides physical data to test the accuracy of SILOGP. In the experiment, one of three ${ }^{60} \mathrm{Co}$ point sources was placed on the axis of a cylindrical concrete silo. Wedges atop the silo collimated the photons into a cone with a full angle of $150.5^{\circ}$, and with the point source at the apex of the cone. Exposure rates were measured and recorded at distances up to $700 \mathrm{~m}$ from the source. A sodium iodide spectrometer measured the differential energy 
spectrum of scattered photons over the same distances, and these results were converted to exposure rates to confirm the values measured directly. In other cases, the collimator was replaced by a concrete shield over the source, either $21 \mathrm{~cm}$ thick or $42.8 \mathrm{~cm}$ thick. Similar measurements were taken under these conditions and recorded. Air density during each source exposure was computed from atmospheric data taken at the time of measurement.

Final exposure rates were corrected for the energy and directional sensitivity of the ionization chamber. For comparisons, all reported exposure data were multiplied by the square of the source-to-detector distance, divided by the solid angle of collimation formed by the silo, and normalized to a source strength of one photon per second. This compensates for inverse-square attenuation and reduces the range of values required hy graphs. To account for variations in air density between measurements, these normalized exposure data were plotted against the areal density, the product of the source-detector distance and the air density.

Three comparison runs of SILOGP were performed, one for the open silo and one for each of the shielded source configurations. The $1.17 \mathrm{MeV}$ and $1.33 \mathrm{MeV}$ photons emitted by ${ }^{60} \mathrm{Co}$ were approximated hy $1.25 \mathrm{MeV}$ photons, a common practice in numerical work. A representative air density of 1.12 $\mathrm{mg} / \mathrm{cm}^{3}$ was chosen, close to many of the air densities reported during the benchmark experiments. Where a concrete shield was required, the measured density of $2.13 \mathrm{~g} / \mathrm{cm}^{3}$ reported hy Roseherry (Ros0) was input to the code.

The results of SILOGP are compared with the experimental results in Fig. 4-1. Notice that SILOGP underpredicts the experimental results below 30 $\mathrm{g} / \mathrm{cm}^{2}(270 \mathrm{~m})$ in all configurations. Roseberry attributed a similar problem 


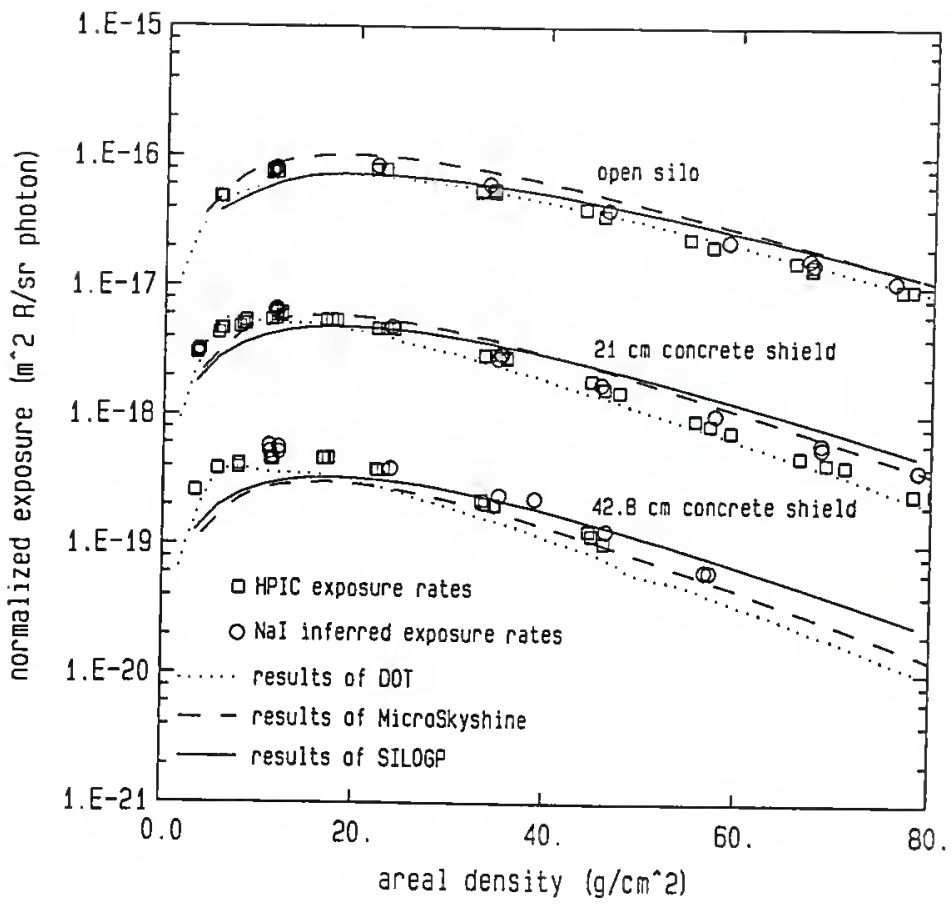

Fig. 4-1. Comparison of measured data from the benchmark skyshine experiment, discrete ordinates calculations by DOT, and calculated results of the point-kernel
code SILOGP. 
with SKY to direct penetration of photons through the concrete silo walls in the experiment. Since SKY did not account for direct penetration, Roseberry expected underprediction below a source-detector distance of $200 \mathrm{~m}$, especially where the source was shielded. (Ro80) SILOGP, however, artificially increases response by allowing photons to scatter in the air within the silo and travel in air directly to the detector, "through" the silo walls. This approximation could compensate for the direct penetration, but it was introduced to simulate photons which scatter for the first time within the confines of the silo, then escape and contribute to dose at the detector. This is separate from the direct penetration suggested by Roseberry. Other non-ideal effects may be present in the physical situation, such as reflection of photons off the silo walls and floor (and roof, if one is present) before they leave the structure; Roseberry indicated that gamma rays could even scatter within the source material or within the stainless steel source containment used in the experiment (Ros0). Most of these phenomena would degrade the photon energy spectrum and increase the detector response close to the silo. Despite the approximation, SILOGP still underestimates the detector response close to the silo, suggesting that the approximation cannot compensate for all these effects.

The code SILOGP overpredicts the experimental results for areal densities above $30 \mathrm{~g} / \mathrm{cm}^{2}$ in all cases. One likely cause for this is the uncertainty introduced by applying an infinite medium buildup factor for an isotropic point source on the second leg of the photon path. Recall from the previous chapter that soil tends to absorb more scattered photons than air, so that the use of an infinite-air buildup factor where an air-ground interface exists will 
cause overprediction of detector response near the ground. The uncertainty increases with distance from the source, as it did with the SKY code (Ro80). Another uncertainty is introduced by the use of a buildup factor for an isotropic point source with the anisotropic scattering source. The actual scattering distribution at the differential volume $\mathrm{dV}$ is biased in the forward direction, with fewer gamma rays scattering back toward the source. The number of photons scattering toward the detector, however, is the same for the actual anisotropic scattering source and the isotropic source implicitly assumed in the use of the buildup factors. If the photon must scatter through a large angle (i.e., if $\beta$ is small), the isotropic source will probably have a smaller total strength than the true scattering source, since the anisotropic scattering source is weaker in backward directions. Conversely, if the photon scatters through a small angle (i.e., $\beta$ is large), the assumed isotropic source is stronger than the actual scattering source, and the use of the buildup factor will probably result in overestimates of detector response. This would help to explain why SILOGP overpredicts experimental results for long source-detector distances; most photons which contribute to dose in these instances would undergo small-angle scatters. SILOGP shares this approximation with WALLGP, MicroSkyshine and G3; MicroSkyshine exhibits the same tendency in Fig. 4-1, supporting this argument.

Overall, the computed results agreed best with the benchmark data for the open silo, ranging from $25 \%$ underprediction to $36 \%$ overprediction. Cases involving concrcte shields did not agree as well with experiment, but in all problems, SILOGP underpredicts at short distances and overpredicts at long distances. The typical deviation of SILOGP from experiment was $20 \%$ for the 
case of the open silo, $40 \%$ for the case of a $21 \mathrm{~cm}$ overhead shield, and $30 \%$ for the case of a $42.8 \mathrm{~cm}$ overhead shield.

After the benchmark study, the discrete ordinates computer code DOT was applied to the benchmark prohlems to test its effectiveness in skyshine studies. While SILOGP is a special-purpose code tailored to this point-source skyshine problem, DOT is more general, incorporating more complex sources and geometries. Both are mainframe codes, but the discrete ordinates approach of DOT requires much more expense and computer resources than the point-kernel method of SILOGP or any other code discussed in this chapter. Normally, DOT is used only for major design prohlems or benchmark calculations, such as the ANSI Standard Problems or the KSU experiment discussed here.

Roseberry (Ro80) reports that DOT computations performed using 39 energy groups closely matched measured exposure rates past $200 \mathrm{~m}$ from the source in an open silo. Computations were also performed with 10 energy groups for all configurations, shielded and unshielded. These results consistently underestimated the benchmark measurements beyond a detector position of $30 \mathrm{~g} / \mathrm{cm}^{2}$ from the source. Nason (Na79) tabulated the results and concluded that neglect of the air-ground interface in the DOT runs led to this underestimate, which averaged $10 \%$ but was as much as $20 \%$ at times. Since SILOGP also neglects ground effects, a comparison may be made between the discrete ordinates method and the single-scatter method.

Figure 4-1 also compares SILOGP results with the DOT results reported by Nason. The two programs agree well where no overhead shield is involved; note that these DOT results were obtained with 39 groups. Where a shield is 
present, a large difference can be seen: 10-group DOT results underpredict benchmark measurements over most of the region of interest, while SILOGP overpredicts. Calculations using 39 groups were not performed with DOT for these cases, though the finer energy structure might have yielded better results. From a conservative view, the single-scatter method would be recommended over discrete ordinates calculations with a coarse energy grid. Close to the silo, the reverse would be true. The discrete ordinates method might also yield better accuracy with a finer energy structure. The advantages of DOT are offset by its extreme cost and high demand on computer resources, however, so that the single-scatter method might be the first choice in actual practice.

Figure 4-1 includes results of the microcomputer program MicroSkyshine for the benchmark experiment. While the code agrees well with benchmark results, the conditions used by MicroSkyshine are not identical to those of the experiment. The MicroSkyshine program will be reviewed alone in a later section, with comparisons to SILOGP made under the conditions of MicroSkyshine.

\subsubsection{Comparisons to ANSI Standard}

The American National Standard ANSI/ANS-6.6.1-1987 (AN87) provides four sets of reference calculations for the validation of measurement methods and numerical techniques. One of these, Reference Problem I.1, was solved by SILOGP to allow an assessment of its accuracy in the limiting case of $2 \pi$ collimation. As with any ANSI Standard, the conditions of the problem are very specific, so that they may be reproduced closely by the method being 
tested. The Standard recognizes that methods are unique; some programs which estimate exposure or dose, for example, cannot separate direct and scattered components in their output. Thus, the Standard recommends that methods be documented, and recommends that assumptions or changes in computer codes which are made to solve the problem be discussed.

In Reference Problem I.1, illustrated in Fig. 4-2, an isotropic point source of $6.2 \mathrm{MeV}$ gamma rays is placed 60 feet $(18.3 \mathrm{~m})$ above the ground, in open air. An imaginary axis runs normal to the ground and through the source, which emits one photon per second. Detectors are placed 3 feet $(0.91 \mathrm{~m})$ above the ground, at distances from the source axis between 200 feet and 5000 feet $(61 \mathrm{~m}$ and $1500 \mathrm{~m})$. Air in the problem has a mass density of $1.22 \mathrm{mg} / \mathrm{cm}^{3}$, with an atomic number density of $1.07 \times 10^{19} \mathrm{atoms} / \mathrm{cm}^{3}$ of oxygen, $4.02 \times 10^{19}$ atoms $/ \mathrm{cm}^{3}$ of nitrogen. Dose rates should be computed in units of $\mathrm{rad}(\mathrm{air}) / \mathrm{year}$, with one year assumed to be 8766 hours.

Since no experimental measurements were available, seven computer codes were selected by the Standards Working Group, and their results for the problem are given as reference values. The methods used include Monte Carlo (OGRE, COHORT II), discrete ordinates transport (DOT-II), the point-kernel method (G33 QADMOD, SKREEN), and integration of parametric airscattering data (SIKYSHINE). Different assumptions were made in each code, and different data libraries were used, but the results agreed with each other, well within an order of magnitude (ANS7, Fa87).

As SILOGP solved this problem, the point source was placed at the top of the silo on its axis, forming a full angle of collimation of $180^{\circ}$. This simulated a source and detector placed at the same height from a ground-air 


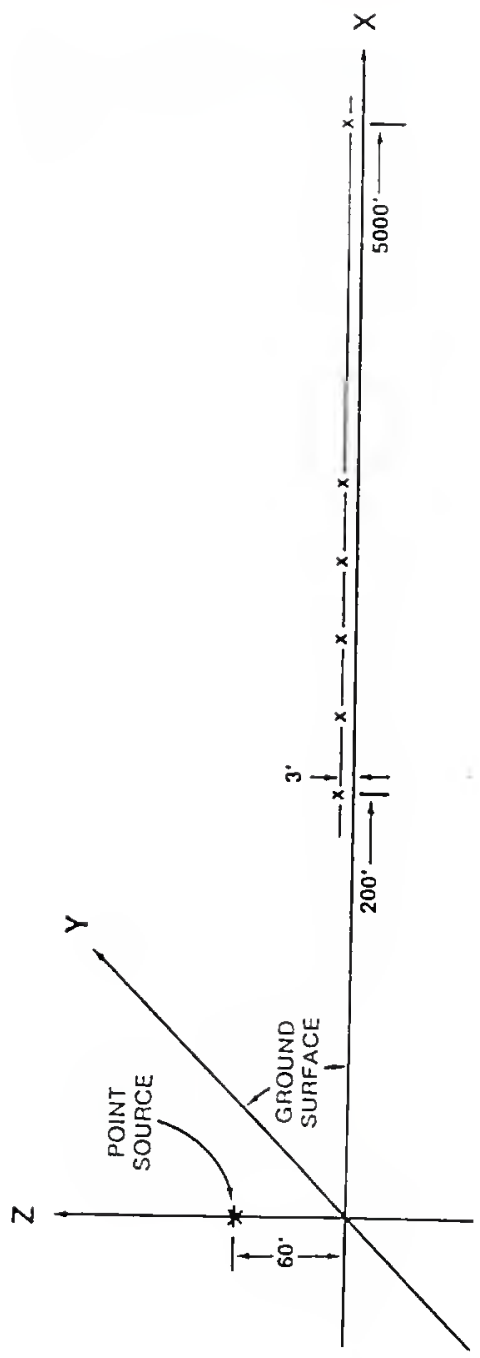

产

点

옹

霖

造它

号 온

它先

\官

은

$\rightarrow$

녕

옥

응

다

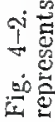


interface with no structures in the vicinity. Since the source and detector of the ANSI reference problem are not the same distance above the ground, the line-of sight source-detector distance in the true problem ranges from 208 feet $(63.4 \mathrm{~m})$ to approximately 5000 feet $(1500 \mathrm{~m})$, not from 200 to 5000 feet exactly. SILOGP computed air kerma starting at $63.4 \mathrm{~m}$ from the source and extending to $1500 \mathrm{~m}$; responses are displayed against the horizontal distance measured along the ground, as required by the Standard, not against the true source-detector distance. Because the ground is treated as air by SILOGP and by several of the codes in the Reference, the air-ground interface can be made parallel to the source-detector axis without introducing error to the results of SILOGP. Also, SILOGP can compute only exposure, kerma, or dose equivalent response. Air kerma is a very good approximation to absorbed dose in air, thus it was used.

In Fig. 4-3, results of SILOGP are compared to the ANSI reference data, and to results of the MicroSkyshine code for Reference Problem I.1. Both programs are in excellent agreement with the reference data and with each other in this limiting case of $2 \pi$ geometry. Of the reference programs, the closest agreement to SILOGP seems to be with the results of the code SKREEN; since both use a point-kernel model, this is not surprising. SILOGP also comes close to the results of $\mathrm{G}^{3}$ at larger distances, and to COHORT II. SILOGP imitates MicroSkyshine most, however; the two codes will be compared extensively later in this section. 


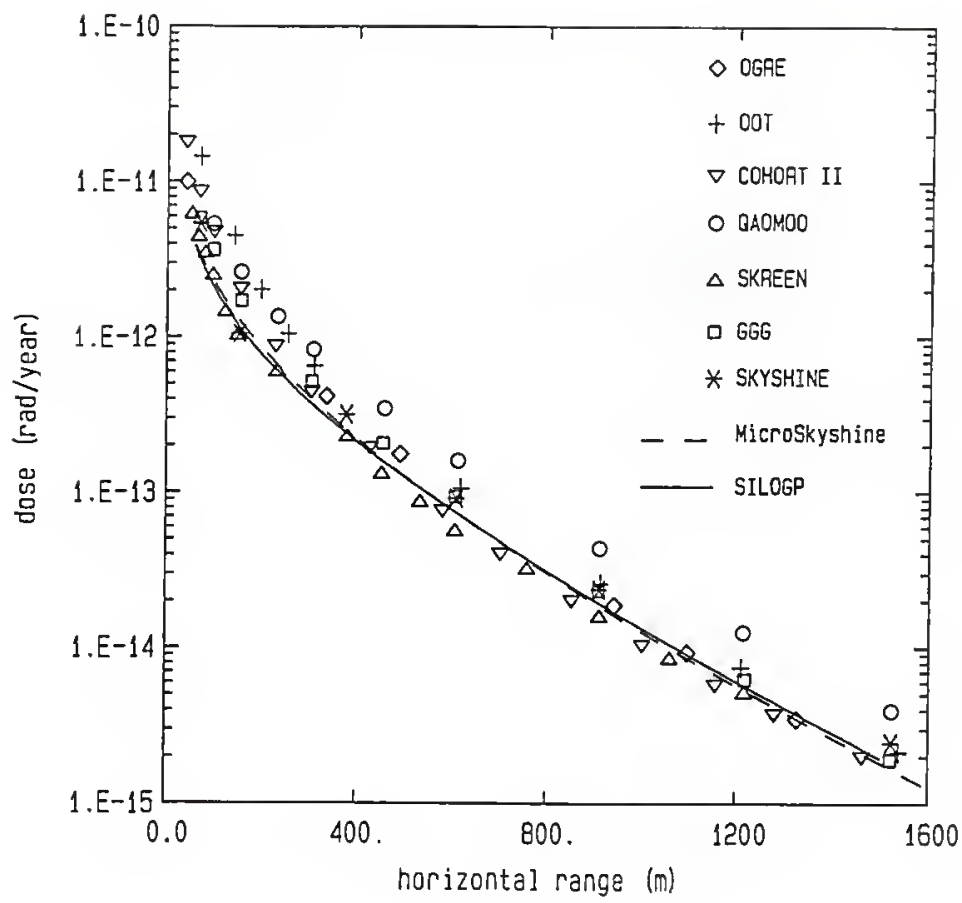

Fig. 4-3. Comparison of SILOGP results for ANSI Reference Problem I.1 to reference values and results of the line-beam response function program
licroSkyshine. 


\subsubsection{Comparisons to Buildup Factors in the $2 \pi$ Problem}

One of the tests used to validate MicroSkyshine (Fa87, Sh87) resembles the ANSI Standard problem but uses buildup factors developed by Chilton, Eisenhauer and Simmons (ChSo) from moments-method calculations. In this inventive benchmark calculation, photons are emitted from a point source into a solid angle of $2 \pi$ steradians. The exposure rate at a point detector due to scattered photons only is determined from the infinite medium buildup factors of Chilton et al. in a straightforward manner.

Consider an isotropic point source emitting $S$ gamma rays of energy $E$ in infinite air, and a point detector located a distance $\mathrm{r}$ from the source. The total response at the detector is an elementary computation:

$$
\mathrm{D}=\frac{S \Re \exp (-\mu \mathrm{r})}{4 \pi \mathrm{r}^{2}} \mathrm{~B}(\mu \mathrm{r})
$$

where

$$
\begin{aligned}
\mathscr{R}= & \text { response function (response per unit fluence) for photons of energy } \\
& \mathrm{E}, \\
\mu= & \text { total attenuation coefficient of photons of energy } \mathrm{E}, \\
\mathrm{B}(\mu \mathrm{r})= & \text { infinite medium buildup factor for photons of energy } \mathrm{E} \text { at } \mu \mathrm{r} \text { mean } \\
& \text { free paths from the source. }
\end{aligned}
$$

This total response arises from both uncollided and scattered photons. The component from uncollided gamma rays is

$$
\mathrm{D}^{0}=\frac{\mathrm{S} \Re \exp (-\mu \mathrm{r})}{4 \pi \mathrm{r}^{2}}
$$

Therefore, in infinite air, the response at the detector due to scattered pliotons alone 
is the difference in these quantities:

$$
\mathrm{D}^{\mathrm{S}}=\frac{\mathrm{S} \mathscr{R} \exp (-\mu \mathrm{r})}{4 \pi \mathrm{r}^{2}}[\mathrm{~B}(\mu \mathrm{r})-1]
$$

However, MicroSkyshine and SILOGP (and WALLGP) are limited to studying the half-space above an air-ground interface. Suppose the point source emits photons in only a hemisphere of directions on one side of a plane, with the source and detector residing in the plane. The detector response for scattered gamma rays in this case would be exactly half the response for the point isotropic source. The result for the $2 \pi$ geometry, therefore, is

$$
\mathrm{D}_{2 \pi}^{\mathrm{S}}=\frac{\mathrm{S} \mathscr{R} \exp (-\mu \mathrm{r})}{8 \pi \mathrm{r}^{2}}[\mathrm{~B}(\mu \mathrm{r})-1]
$$

Since SILOGP computes only the scattered response, and is restricted to the $2 \pi$ geometry, this formula is of interest to us.

The reference calculations for this problem were performed using Eq. (4-4), air kerma buildup factors of Chilton et al. (Ch80) and the attenuation data of Storm and Israel (St67). Exposure rates were computed, thus the response function for exposure was substituted for $\mathscr{R}$ (see section 3.2 of this work). Although the buildup factors are based on air kerma, Chilton et al. state: "The air kerma data can be used for exposure buildup factors to a close approximation, as long as bremsstrahlung is negligible, a matter that...is questionable at the higher energies." (Ch80) These benchmark calculations are for source energies from 0.1 to $10 \mathrm{MeV}$ and distances out to $2500 \mathrm{~m}$ from the point source. Buildup factors are available for $15 \mathrm{MeV}$ photons, 
but were not used in the reference calculations, probably because of the warning quoted above.

The reference results are presented in Figs. $4-4$ and $4-5$ as individual data points, along with SILOGP and MicroSkyshine results for the same problems. The agreement of SILOGP with the buildup results is excellent over the range of photon energies and distances considered. Except for exposures from $0.1 \mathrm{MeV}$ photons beyond $600 \mathrm{~m}$ from the source, the SILOGP results are consistently within $15 \%$ of the buildup values. The improved version of MicroSkyshine, by comparison, overestimates the buildup results past $1000 \mathrm{~m}$, at all energies (Sh87); comparison of Figs. $4-4$ and $4-5$ will demonstrate this.

MicroSkyshine and SILOGP both underestimate the reference results for 0.1 $\mathrm{MeV}$ photons past $600 \mathrm{~m}$. One possible, though unlikely, cause is that both SILOGP and MicroSkyshine use the geometric-progression buildup formula of Harima et al. (Has6). For air, however, the GP coefficients are based on the same data from Chilton et al. as was used in the reference calculations. The maximum deviation of the geometric-progression fit from the data is less than $3 \%$ (RS86) over a range of $40 \mathrm{mfp}$, or about $2000 \mathrm{~m}$. While the fit itself may not be to blame, it is possible that some other influence related to the buildup calculation created the discrepancy. An error or uncertainty in the reference calculations themselves may also be responsible; the cause is not obvious at this time.

\subsubsection{Comparisons to MicroSkyshine}

The MicroSkyshine code (Fa87, Sh87), developed for microcomputers, is applicable to many problems involving skyshine from a point gamma-ray source. Among the problems which it solves are the two simplified geometries studied in 


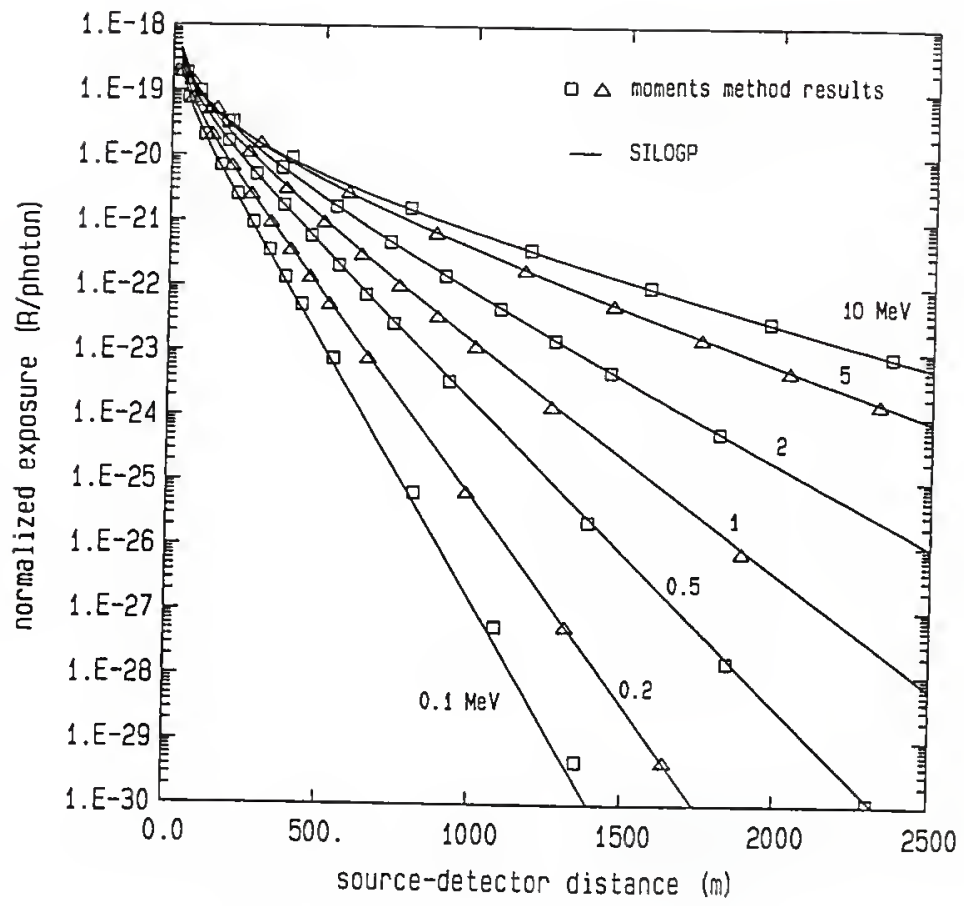

Fig. 4-4. Comparison of SILOGP results for the $2 \pi$ problem of Faw and Shultis (Fa87) to the moments-method reference values. 


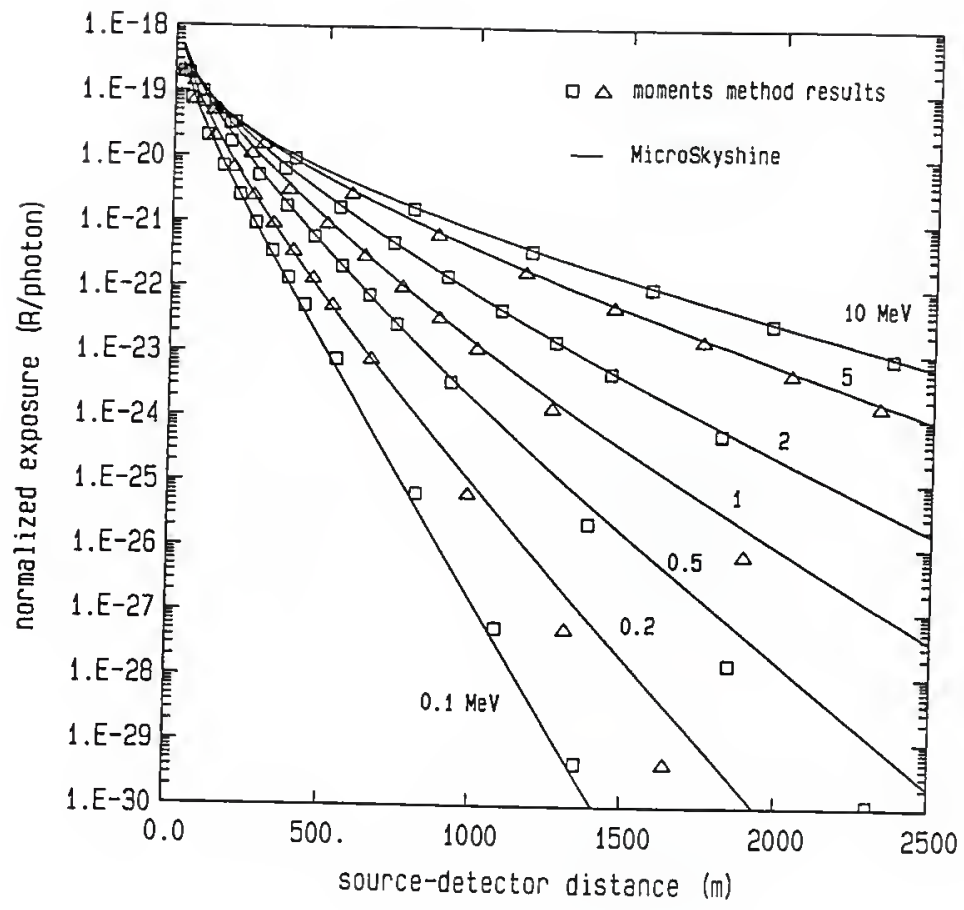

Fig. 4-5. Comparison of MicroSkyshine results for the $2 \pi$ problem of Faw and Shultis (Fa87) to the moments-method reference values. 
this work: the point source on the axis of a cylindrical silo, and the point source behind a semi-infinite wall. The author knows of no other codes which solve these identical problems, save those of this thesis. Thus, a comparison of the results of MicroSkyshine and SILOGP is mandatory.

Differences should be expected when comparing results of the two codes. MicroSkyshine is a general purpose program intended for complex photon sources and geometries; by preparing this line-beam code for microcomputers, the developers exchanged precision for speed of results. It should be emphasized that MicroSkyshine has purposely been made conservative (Sh87), since it is intended for general problems in industrial design. Of all the codes reviewed here, MicroSkyshine is also the least expensive to use. SILOGP, by comparison, is a special-purpose point-kernel code for mainframes which is expensive to use but does not intentionally overpredict detector response and does not sacrifice precision. These facts should be kept in mind as the reader reviews this section.

The first source-detector arrangement of interest is nearly identical to the benchmark problem performed at the KSU Shielding Facility (Na81). A ${ }^{60} \mathrm{Co}$ point source is placed on the axis of a cylindrical silo; the source and the edge of the silo form a line at an angle of $75.25^{\circ}$ from the axis of the silo, so that the full angle of collimation is $150.5^{\circ}$. The ${ }^{60} \mathrm{Co}$ source is approximated by a source which emits $1.25 \mathrm{MeV}$ photons. Exposures per photon are computed at distances out to $700 \mathrm{~m}$ from the source, and normalized in the same fashion as the benchmark data. Calculations are also performed with concrete shields of thickness $21 \mathrm{~cm}$ and 42.8 $\mathrm{cm}$ over the silo. The major difference between the MicroSkyshine problem and the benchmark experiment is in material densities. While Roseberry (Ros0) reported no air densities during the benchmark experiments above $1.21 \mathrm{mg} / \mathrm{cm}^{3}$, the 
MicroSkyshine calculations were performed using an air density of $1.25 \mathrm{mg} / \mathrm{cm}^{3}$ (Fa87). The concrete shields used in the experiment had a reported density of 2.13 $\mathrm{g} / \mathrm{cm}^{3}$ (Ros0), yet calculations performed in the MicroSkyshine validation employed $2.32 \mathrm{~g} / \mathrm{cm}^{3}$ as the concrete density. The differences are not explained in the MicroSkyshine reference; to eliminate disagreement due to differences in material densities, it was decided to perform SILOGP calculations with the densities used in MicroSkyshine.

The results of SILOGP and MicroSkyshine for this problem are displayed in Fig. 4-6. Also shown are the results of the point kernel code $\mathrm{G}^{3}$, as reported by Faw and Shultis (Fa87) for the same problem geometry. The code $\mathrm{G}^{3}$ employs the same point-kernel model and approximations as SILOGP (and WALLGP), but is a general purpose program for design work, and somewhat expensive to run. The specialized codes of this work may be preferable, as $\mathrm{G}^{3}$ uses a combinatorial geometry (RS85) which is very difficult to use. The tendency of $\mathrm{G}^{3}$ to underpredict where a concrete shield is present is made obvious in the plot.

SILOGP yields a lower estimate of exposure than MicroSkyshine for source-detector distances under $500 \mathrm{~m}\left(60 \mathrm{~g} / \mathrm{cm}^{2}\right)$; the codes agree very well for larger distances. In the worst cases, SILOGP results are less than $70 \%$ of the MicroSkyshine estimates. Both programs account for photons which scatter within the silo before leaving it; although this is cited as a major approximation of MicroSkyshine, it cannot be to blame for the discrepancy. MicroSkyshine includes pair production photons in the dose, while SILOGP does not; however, because the gamma rays from ${ }^{60} \mathrm{Co}$ are not far above the pair production threshold, this is not a likely cause either. 


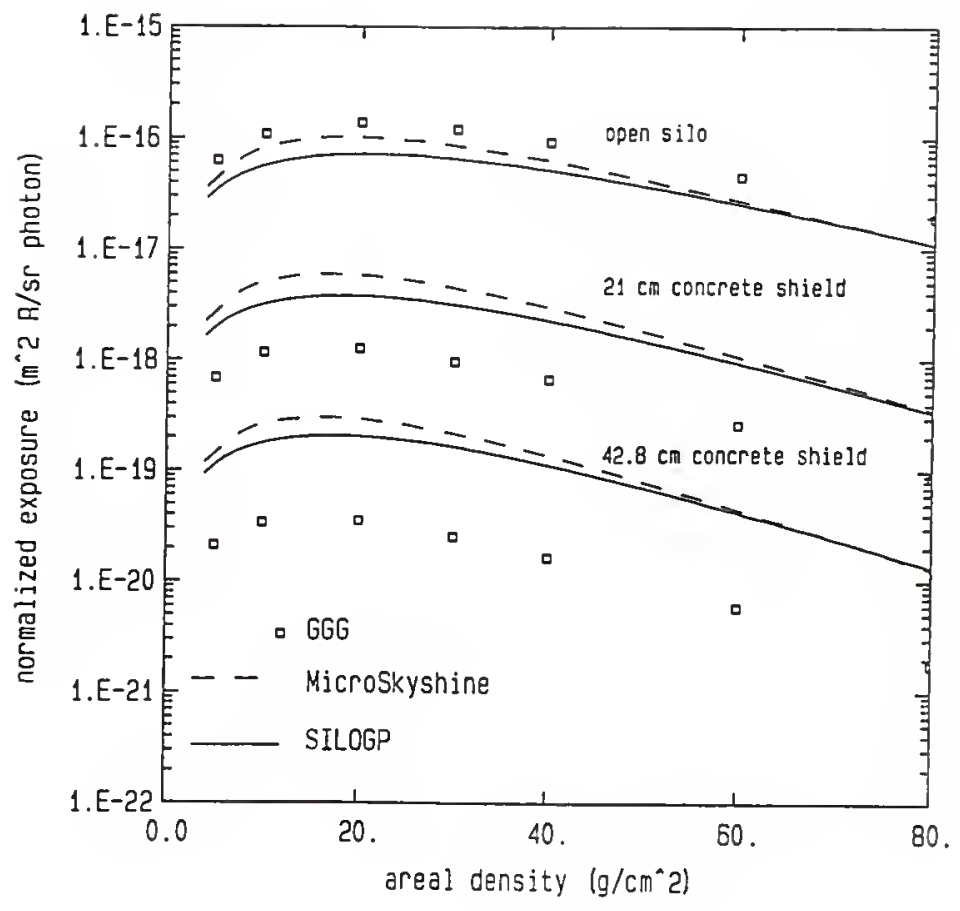

Fig. 4-6. Comparison of results for the benchmark problem computed by MicroSkyshine, $G^{3}$ and SILOGP. In this comparison, air density is $1.25 \mathrm{mg} / \mathrm{cm}^{3}$, and concrete density is $2.32 \mathrm{~g} / \mathrm{cm}^{3}$. 
A similar disagreement hetween SILOGP and MicroSkyshine is evident in the preceding discussion of the moments-method $2 \pi$ prohlem. MicroSkyshine computes higher exposure rates than SILOGP for all the prohlems in Figs. 4-4 and 4-5, especially past $1000 \mathrm{~m}$. Since the two geometries involve different angles of collimation, it was decided to compare the responses computed hy the codes over a larger range of collimation angles. Any tendencies shown in this parametric study could help to explain the discrepancies seen in other prohlems. For a point source of $1 \mathrm{MeV}$ photons on the axis of an open silo, in air of density $1.25 \mathrm{mg} / \mathrm{cm}^{3}$, the exposure per photon was computed hy hoth codes at a detector $400 \mathrm{~m}$ from the source. Collimation angles formed hy the silo ranged from $179.999^{\circ}$ (approaching the $2 \pi$ prohlem) to $1^{\circ}$ (approaching a line beam directed normal to the source-detector axis). The results are presented in Tahle 4-1. The difference in results decreases as the collimation "opens up"; for the case approaching a line-heam source, the SILOGP result is $15 \%$ lower than that of MicroSkyshine, hut for the $2 \pi$ case, the difference is only $9 \%$.

An important clue to the cause can he found in the second KSU report hy Shultis and Faw on MicroSkyshine (Sh87). The MicroSkyshine method employs line-heam response functions, evaluated at selected gamma-ray energies and beam angles from the source-detector axis. The response functions were computed hy a point-kernel formula similar to Eq. (3-9) of this work, hut including pair production in the evaluated response. A formula involving three parameters was fit to these response functions, and hy interpolating the parameters, the response functions can he evaluated continuously over energy and beam angle. This formula, not the point-kernel equations which generate the response functions, is evaluated hy MicroSkyshine in computing exposures or doses; it is identical to the parametric 
Table 4-1. Comparison of detector responses computed by the codes MicroSkyshine and SILOGP with changes in collimation angle. Values of exposure per photon are presented for a point source of $1.0 \mathrm{HeV}$ photons placed inside an open silo of radius $1 \mathrm{~m}$. The detector lies $400 \mathrm{~m}$ from the source in each case, on a line normal to the silo axis. The height of the silo above the source defines the angle of collimation. Air density is taken as $1.25 \mathrm{mg} / \mathrm{cm}^{3}$. All SILOGP results were obtained using a convergence criterion of $1 \%$.

\begin{tabular}{|c|c|c|c|c|}
\hline \multirow{2}{*}{$\begin{array}{l}\text { Silo } \\
\text { height } \\
\text { (m) }\end{array}$} & \multirow{2}{*}{$\begin{array}{l}\text { Full angle } \\
\text { of } \\
\text { collimation } \\
\text { (degrees) }\end{array}$} & \multicolumn{2}{|c|}{ Normalized exposure ( $\mathrm{R} / \mathrm{photon}$ ) } & \multirow{2}{*}{$\begin{array}{l}\text { Percent } \\
\text { difference } \\
\text { from Micro- } \\
\text { Skyshine }\end{array}$} \\
\hline & & $\begin{array}{l}\text { Computed by } \\
\text { MicroSkyshine }\end{array}$ & $\begin{array}{l}\text { Computed by } \\
\text { SILOGP }\end{array}$ & \\
\hline $\begin{array}{c}0.00001 \\
0.01 \\
0.1 \\
0.7 \\
1.0 \\
5.0 \\
114.589\end{array}$ & $\begin{array}{l}179.999 \\
178.854 \\
168.6 \\
110 \\
90 \\
22.62 \\
1\end{array}$ & $\left.\begin{array}{l}2.853(-21)^{*} \\
2.725 \\
1.929 \\
2.21 \\
2.941 \\
1.524 \\
1.21 \\
6.160 \\
1.179 \\
1.22 \\
-24\end{array}\right)$ & $\begin{array}{l}2.605(-21) \\
2.478(-21) \\
1.737(-21) \\
2.542(-22) \\
1.312(-22) \\
5.279 \\
1.003(-24)\end{array}$ & $\begin{array}{l}-8.7 \\
-9.1 \\
-10.0 \\
-13.6 \\
-13.9 \\
-14.3 \\
-15.0\end{array}$ \\
\hline
\end{tabular}

$*^{*} 2.853(-21)=2.853 \times 10^{-21}$ 
fit used in the SKYSHINE-II program, the industry-standard mainframe code at the time MicroSkyshine was created (Fa87, Sh87).

In the MicroSkyshine report, Shultis and Faw report the deviations of the fits from the doses computed by the point-kernel method. The mean absolute deviation (MAD) and maximum deviation of the fit over the range of source-detector distances are tabulated in the report for each energy and beam angle, and patterns are evident. For the gamma-ray energies above $1.5 \mathrm{MeV}$, the MAD peaks at about $10 \%$ at an angle of $75^{\circ}$ or $85^{\circ}$, decreasing as the angle increases or decreases from this direction. At $1.5 \mathrm{MeV}$, the MAD of the parametric fit increases with the beam angle to $11 \%$ at $75^{\circ}$, decreases as the beam angle increases, then rises again; as the beam approaches the direction opposite the source-detector axis, the formula can deviate from the computed response as much as $50 \%$. At $0.75 \mathrm{MeV}$, the MAD reaches a maximum at a beam angle of $85^{\circ}$, then remains at this level; the three-parameter formula typically deviates by 12 or 13 percent, sometimes by as much as $35 \%$ above the point-kernel results. Below $0.75 \mathrm{MeV}$, the fit deviates even more from the computed response, fits worst at angles as low as $25^{\circ}$ or $35^{\circ}$, and can be as much as $45 \%$ above the point-kernel values. Data computed over a source-detector range of $2500 \mathrm{~m}$ or more produced each set of coefficients, and the report states that the fit is almost always worst when the detector is closest to the source.

In Figs. 4-4 and 4-5, the greatest disagreement between SILOGP and MicroSkyshine occurs between 0.1 and $1.0 \mathrm{MeV}$, where the parametric fits of the latter code are most inaccurate. In Fig. 4-6, the discrepancies between MicroSkyshine and SILOGP are worst below $60 \mathrm{~g} / \mathrm{cm}^{2}$, or $480 \mathrm{~m}$; compared to the $2500 \mathrm{~m}$ range of the fits, this is close to the source, and the poor fit by 
MicroSkyshine close in would help to explain the disagreement. If the point-kernel results of SILOGP are nearly equal to the point-kernel results to which the MicroSkyshine formulas are fit, this disagreement would be entirely due to the uncertainties produced by the fit.

The pattern seen in Table 4-1 can also be explained by the deviation between the fitted response function of MicroSkyshine and the computed doses upon which it is based. In the table, $1 \mathrm{MeV}$ photons are collimated through angles from $1^{\circ}$ to $180^{\circ}$, with the discrepancy between SILOGP and MicroSkyshine decreasing with increasing collimation angle. The uncertainty in the MicroSkyshine function fit for beam angles less than $90^{\circ}$ is likely to fall below that at $90^{\circ}$ if the source photons are between $0.75 \mathrm{MeV}$ and $1.5 \mathrm{MeV}$; also, the interpolation between these energies would produce the largest uncertainty in the computed dose at large beam angles, those above $90^{\circ}$. A one-degree collimation angle in MicroSkyshine would include only line-beams at an angle of $90^{\circ}$ to the source-detector axis, while a $2 \pi$ problem would include the beams at smaller and larger angles. Assuming photons which leave the source in directions toward the detector have more bearing on the detector response than photons heading away from the detector, it would be possible for the differences between the fitted function results of MicroSkyshine and the "true" results of the moments-method doses to decrease as the collimation angle increases and the function is evaluated at larger and smaller angles. This is the same trend shown in the last column of Table 4-1.

In summary, because the uncertainties of the line-beam gamma-ray response functions created for use in MicroSkyshine show the same behavior as the differences between MicroSkyshine and SILOGP, this author concludes that the inaccuracies of those functions are responsible for their disagreement. It is possible that a function 
which reproduces the original dose data more faithfully would produce results more in line with SILOGP, since the dose data were obtained with a point-kernel calculation very similar to the methodology of SILOGP.

\subsection{Comparison of WALLGP to Other Methods}

The remainder of the chapter will concentrate on WALLGP, the code produced specifically for this tbesis. No benchmark experiment has been performed for the problem of a gamma-ray source behind a perfectly absorbing wall, so a definitive validation of the results of WALLGP is not possible. It is possible to test the accuracy of the code in the limiting case of a $2 \pi$ geometry; the ANSI Standard problem and the $2 \pi$ benchmark calculation prepared by Faw and Shultis (Fas7, Sh87) can be compared to WALLGP results for a geometry involving a very low wall, nearly a $2 \pi$ solid angle of collimation for source photons. The ANSI Standard problem could be considered important in validating WALLGP, but it does not involve a wall, the pbysical arrangement WALLGP was intended for. MicroSkyshine is the only other code known to this author which can predict detector responses from sources concealed behind a semi-infinite wall; WALLGP and MicroSkyshine will be compared extensively in tbis section. WALLGP will be applied to many of tbe problem geometries presented in tbe MicroSkyshine documentation (Fa87, Sh87), since results are readily available for the latter code. Differences in the codes will also be analyzed as functions of source photon energy, overhead shield thickness, and wall height; this parametric study may help to explain differences in computed exposures and doses, just as the studies for SLLOGP did. 


\subsubsection{Comparisons to ANSI Standard}

In the extreme case of an unshielded, uncollimated source geometry, ANSI Standard Problem I.1, which was described in section 4.1.2, is available for comparisons with WALLGP. This configuration of source and detector is an extreme case, not one for which WALLGP was intended, but it can verify the single-scatter model upon which the code is based. Limiting cases are often excellent tests of the accuracy of a program and its underlying theory; other codes have been adapted to a problem involving only a point source and point detector for such tests, although the codes were meant for more complex situations (Ma69).

To approximate a point source in infinite air with the semi-infinite wall problem, the wall must be effectively removed from the geometry; the source and detector should be placed so that the line of sight between the two would graze the top of the wall. This would be similar to placing the source and detector on the ground with only infinite air above. SILOGP could effectively remove the obstruction by placing the source and detector at the level of the silo opening. WALLGP cannot accomplish this, however, because some limits of integration are inversely proportional to the height $\mathrm{h}$ of the wall above the source-detector axis; when the source-detector axis rests on the wall edge, the limits are no longer valid. The point source in open air must be approximated by a source barely hidden from the detector by the wall. For all WALLGP validation runs, the point source and detector lie $.01 \mathrm{~mm}$ below the edge of the wall. All other problem specifications are adhered to, such as the photon source energy of $6.2 \mathrm{MeV}$ and the air density of 1.22 $\mathrm{mg} / \mathrm{cm}^{3}$. A cutoff value of 40 mean free paths was used, and the convergence criterion DEL was set to produce an answer with estimated error of $0.1 \%$. 
The source and detector of the ANSI reference problem are not the same distance above the ground, recall; air kerma responses have been computed by WALLGP using the true source-detector separation distance, then displayed against the horizontal distance from the detector to a point directly below the source. It was again assumed that the direction of the surface between the air and the ground makes no difference in the comparison, since the ground is treated as air by WALLGP and by several of the ANSI codes.

The doses predicted by WALLGP for the ANSI Standard Problem are shown in Fig. 4-7 against the ANSI reference data and the results of MicroSkyshine. The agreement with the Standard is excellent, except for the closest detector points, those within $100 \mathrm{~m}$ of the ground point below the source. The error criterion of $0.1 \%$ is not adequate at this close range; recall from Chapter 3 tbat the error criterion must be selected carefully in tbis region. Here, increasing tbe cutoff adds negligible values of exposure and complicates the evaluation of tbe integral exposure rate equation. Beyond $100 \mathrm{~m}$, the results of WALLGP are nearly identical to those of SILOGP presented earlier; this is to be expected, since both use the same point-kernel model, and tbe same equation is integrated over nearly identical regions in botb programs. The estimates made by MicroSkyshine and WALLGP are almost a single curve beyond $100 \mathrm{~m}$ of the ground point below the source. This too was evident with SILOGP, and validates both the point-kernel model and the line-beam response function method for ANSI Standard Problem I.1.

\subsubsection{Comparisons to Buildup Factors in tbe $2 \pi$ Problem}

The $2 \pi$ problem developed by Faw and Shultis (Fas7, Sh87) for testing MicroSkyshine can be modeled exactly by SILOGP, but not by WALLGP; as with 


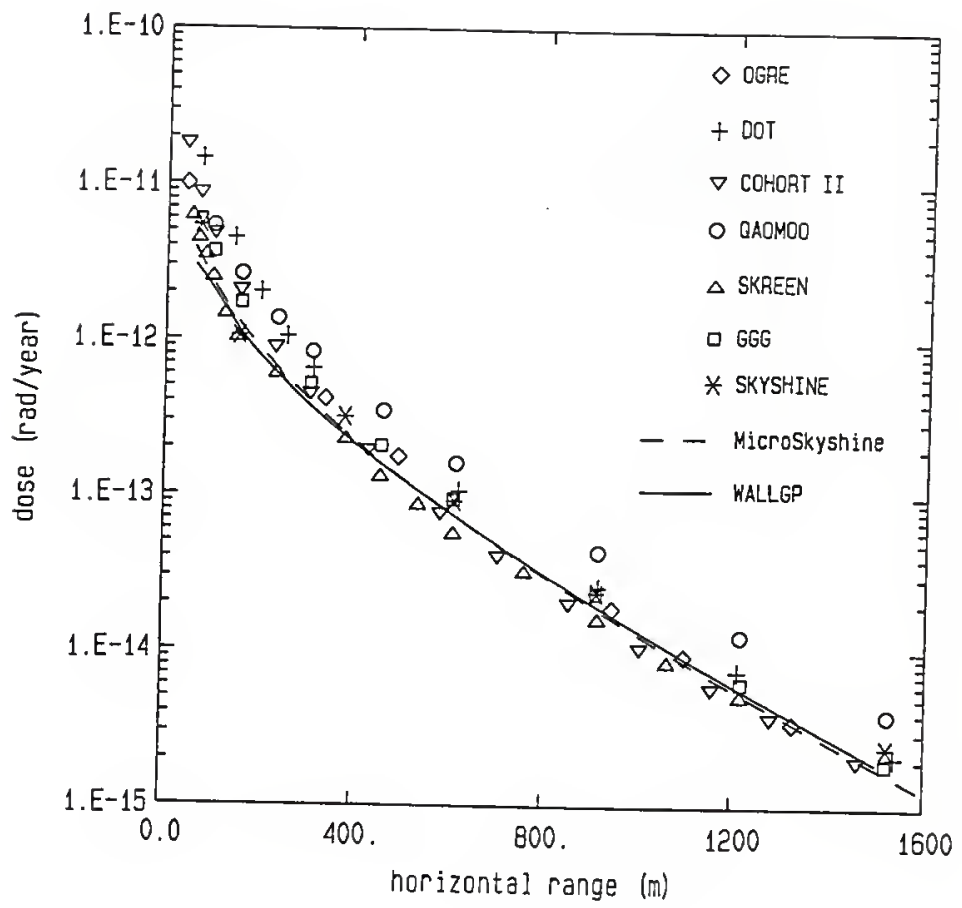

Fig. 4-7. Comparison of WALLGP results for ANSI Reference Problem I.1 to reference values and results of the line-beam response function program
ificroShyshine. 
the ANSI Standard problem, the line-of-sight of the source and detector must be blocked by the wall for the limits of integration used by WALLGP to be valid. The problem is described in section 4.1.3, and involves a point source and point detector resting on an air-ground interface. By definition of the $2 \pi$ problem, photons must leave the source in the direction of the air. To create as closely as possible the hemisphere of directions in which photons leave the source, the height $\mathrm{h}$ of the wall above the source-detector axis is made a small "epsilon" value, $.01 \mathrm{~mm}$. Exposure rates are computed out to $2500 \mathrm{~m}$ from the source, for photons of energies ranging from 0.1 to $10 \mathrm{MeV}$.

The results of WALLGP are compared to the moments-method reference values in Fig. 4-8. At all energies except $0.1 \mathrm{MeV}$, WALLGP fits the reference points closely; where percent differences can be judged, WALLGP comes within $15 \%$ of reference values. The program underpredicts the dose computed by buildup factors at the lowest energy, $0.1 \mathrm{MeV}$; as stated in the validation of SILOGP, the cause of this disagreement is not obvious. As expected, SILOGP and WALLGP produce nearly identical curves in Figs. $4-4$ and $4-8$, because the two programs employ the same model and integrate the same formula over nearly identical regions of space. Based on the results for the ANSI Reference Problem and the $2 \pi$ geometry, WALLGP and SILOGP are valid for use in $2 \pi$ configurations where photons are uncollimated or nearly so, that is, where a silo or wall barely blocks the source from the detector and photons travel in nearly all directions from the source above ground. 


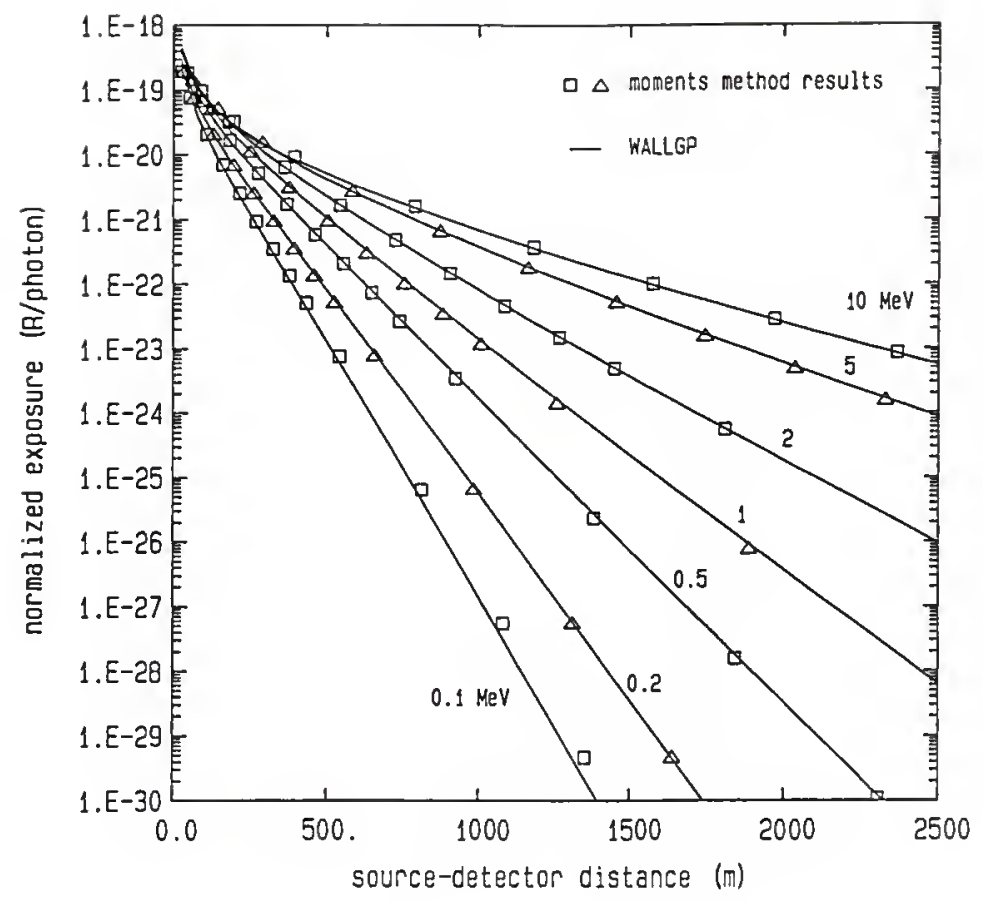

Fig. 4-8. Comparison of WALLGP results for the $2 \pi$ problem of Faw and Shultis (FaS7) to the moments-method reference values. 


\subsubsection{Comparisons to MicroSkyshine}

The remainder of this chapter is concerned with MicroSkyshine and WALLGP, and their computed responses for a variety of problems. The author knows of no other computer codes which address the ideal problem of a semi-infinite wall between source and detector; it is natural that the two codes be contrasted over a wide range of source-detector configurations. Most of the work presented here is in the form of parametric comparisons, discussions of how results change with shifts in problem parameters. Since the two codes do not share certain assumptions, differences will be evident; the parametric comparisons are intended to demonstrate the causes for disagreement.

The first problem of this set was chosen from the reports on MicroSkyshine (Fa87, Sh87) and is the class of problem WALLGP is most concerned with. In this situation, a ${ }^{16 \mathrm{~N}}$ point source, emitting $6.13 \mathrm{MeV}$ gamma rays, is one meter from the face of a semi-infinite wall of shielding material. A point detector is placed on the other side of the wall, at distances up to $800 \mathrm{~m}$ from the source. Both the source and detector are $0.7 \mathrm{~m}$ below the top edge of the wall, as measured parallel to the wall face, and the axis between the source and detector is perpendicular to the wall. For one case, no shielding is above the source, and in the second, a concrete shield $30 \mathrm{~cm}$ thick rests on the top of the wall and extends over all space on the source side of the wall. The air medium has a density of $1.25 \mathrm{mg} / \mathrm{cm}^{3}$, while concrete shielding, If used, has a density of $2.13 \mathrm{~g} / \mathrm{cm}^{3}$. For purposes of accuracy, the problem was run by WALLGP using a mean free path cutoff of $40 \mathrm{mfp}$ and an error criterion of 0.005 $(0.5 \%$ error in the result).

The dose estimates obtained from MicroSkyshine and WALLGP are illustrated in Fig. 4-9. The results of the single-scatter code $\mathrm{G}^{3}$ are also shown. As 


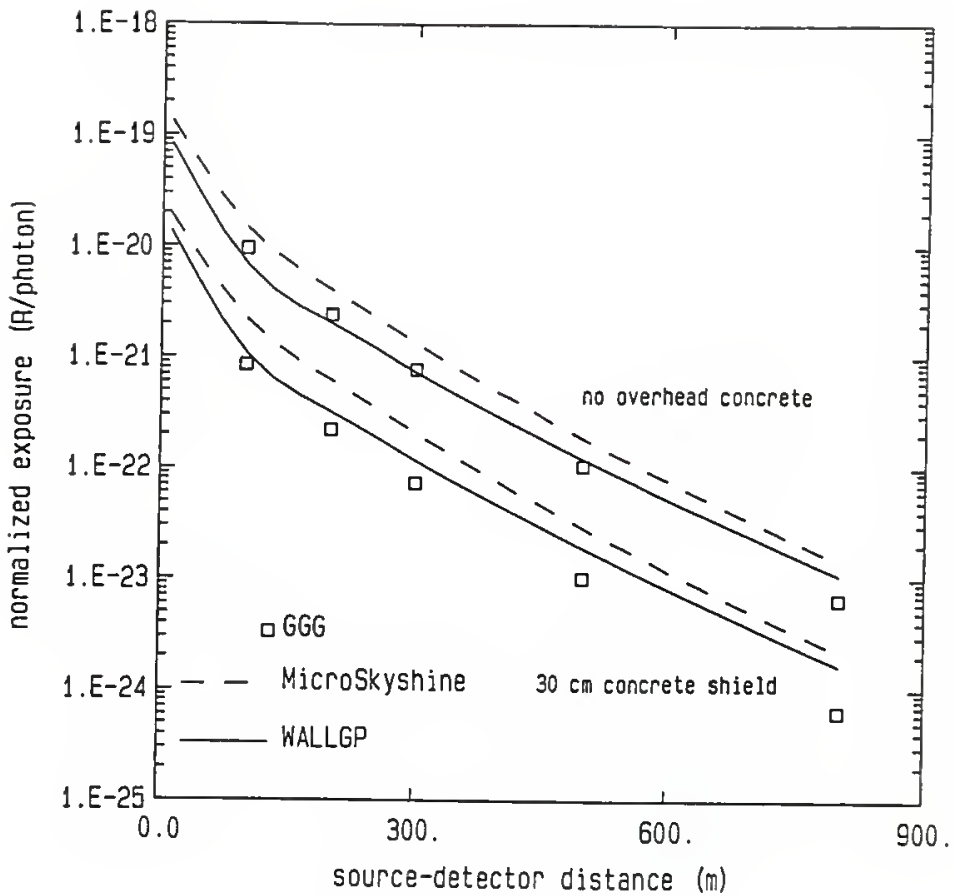

Fig. 4-9. Comparison of dose estimates computed by MicroSkyshine, $\mathrm{G}^{3}$ and WALLGP. A ${ }^{16} \mathrm{~N}$ point source is located $1 \mathrm{~m}$ behind a wall in an infinite air medium, with the source and detector on a line normal to the wall and $0.7 \mathrm{~m}$ below its top edge. Where a concrete shield is present, it rests on the top of the wall and extends over the source, at a right angle to the wall. 
mentioned in the MicroSkyshine report (Fa87), G ${ }^{3}$ cannot account for buildup effects in the overhead shielding, and seriously underpredicts the detector response in all cases involving overhead shielding. This is evident in the figure, where $\mathrm{G}^{3}$ results for the case of $30 \mathrm{~cm}$ of overhead shielding are below values of both MicroSkyshine and WALLGP. The point-kernel code WALLGP consistently computes a significantly lower exposure than MicroSkyshine over the entire measurement baseline; since MicroSkyshine is intentionally conservative, this is not surprising. The difference of WALLGP ranges from $20 \%$ below MicroSkyshine results at the extreme distances to $50 \%$ below MicroSkyshine at a source-detector distance of $100 \mathrm{~m}$. The presence or absence of the concrete shield does little to change this disagreement, suggesting that it is not involved.

Three causes for the disagreement are evident. The first was discussed in the comparisons of SILOGP and MicroSkyshine, but did not cause difficulties with SILOGP. To account for photons which scattered from the walls of the silo during the benchmark experiment and contributed to measured dose, MicroSkyshine uses the silo, walls, or other shielding only to collimate the photon source. Only line beams which do not pass through the silo or wall are included by MicroSkyshine in computing dose; response from the entire beam is included, even from those photons which would scatter behind the wall or within the silo and pass through the obstruction to reach the detector (Fa87). SILOGP makes a similar approximation to produce results closer to the benchmark measurements. WALLGP does not compute dose using gamma rays which must pass through the wall, however; photons which scatter from the wall face are much less likely to contribute to detector dose than photons which reflect from the back of the silo, and the approximation is not as helpful. Figure $4-10$ is a graphic comparison of the 


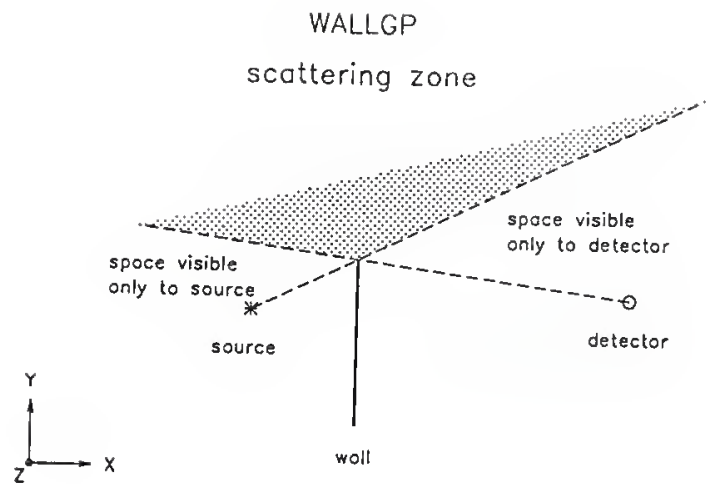

MicroSkyshine

scattering zone

zone visible

to detector

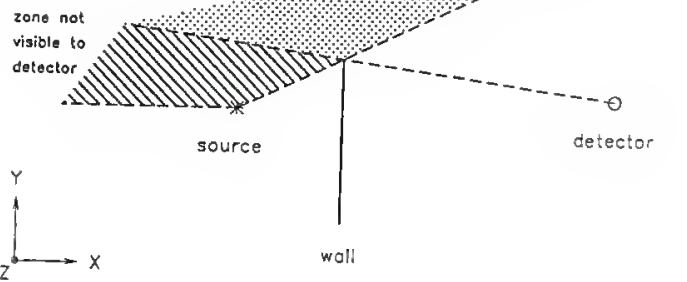

Fig. 4-10. Comparison of the regions of space in the wall geometry in which WALLGP and MicroSkyshine allow photons to undergo their first scatter. In IVALLGP, the point of scatter must have a clear path to both the source and detector. In MicroSkyshine, line beams from the source must not intersect the wall and photons may scatter anywhere along a line beam, including points obstructed
from the detector. 
scattering zone of WALLGP with the approximated scattering zone of MicroSkyshine, showing the areas behind the wall where the photon is allowed to scatter for the first time. MicroSkyshine's overpredictive results should be higher than those of WALLGP, since MicroSkyshine makes the approximation in all problems, including the wall case.

The second cause of disagreement is pair production. MicroSkyshine has included annihilation photons in its response functions (Sh87), while WALLGP ignores pair production. For $6.13 \mathrm{MeV}$ photons, the pair production cross-section is not negligible, and WALLGP can be expected to underestimate the detector response to a ${ }^{16} \mathrm{~N}$ source. The third possible cause for a difference in results from WALLGP and MicroSkyshine is the deviation of the fitted response function curves used in MicroSkyshine from the point kernel data used to produce the fits. Shultis and Faw (Sh87) report adequate fits with the coefficients at $5.5 \mathrm{MeV}$ and $6.5 \mathrm{MeV}$, from which responses to $6.13 \mathrm{MeV}$ photons are computed; the largest deviation of the fitted formulas from the data is under $17 \%$. If the formulas tend to overpredict the original point-kernel data, and those data agree well with the point-kernel results of WALLGP, this would combine with the inclusion of pair production to widen the disagreement of the two codes. MicroSkyshine would be likely to overpredict, while WALLGP, because of its neglect of pair production, underpredicts.

The next comparison between the two programs is presented in Table 4-2. The source, detector and wall are configured as they were for the problem of Fig. 4-9, except that the detector remains at $400 \mathrm{~m}$ from the source, and the energy of source photons is varied from 1.0 to $8.0 \mathrm{MeV}$. The two programs diverge as the source energy increases, and at $8 \mathrm{MeV}$, MicroSkyshine reports almost double the 
Table 4-2. Comparison of detector responses computed by the codes MicroSkyshine and WALLGP with changes in source energy. Values of exposure per photon are presented for a point source placed $1 \mathrm{~m}$ behind a semi-infinite wall and $0.7 \mathrm{~m}$ below the top of the wall. The detector lies $399 \mathrm{~m}$ from the wall face opposite the source, and $0.7 \mathrm{~m}$ below the top of the wall. The source-detector axis is normal to the wall. Air density is $1.25 \mathrm{mg} / \mathrm{cm}^{3}$; concrete density is $2.13 \mathrm{~g} / \mathrm{cm}^{3}$. All WALLGP results were obtained using a convergence criterion of $1 \%$ and a cutoff distance of $40 \mathrm{mfp}$.

\section{Normalized exposure ( $\mathrm{R} /$ photon)}

\begin{tabular}{llll}
$\begin{array}{l}\text { Source energy } \\
\text { (MeV) }\end{array}$ & $\begin{array}{l}\text { Computed by } \\
\text { MicroSkyshine }\end{array}$ & $\begin{array}{l}\text { Computed by } \\
\text { WALLGP }\end{array}$ & $\begin{array}{l}\text { Percent difference } \\
\text { from MicroSkyshine }\end{array}$ \\
\hline
\end{tabular}

No overhead shield

$\begin{array}{llll}1.0 & 4.204(-22) * & 3.606(-22) & -14.2 \\ 2.0 & 4.785(-22) & 4.011(-22) & -16.2 \\ 3.0 & 4.755(-22) & 3.816(-22) & -19.7 \\ 4.0 & 4.732(-22) & 3.497(-22) & -26.1 \\ 5.0 & 4.746(-22) & 3.204(-22) & -32.5 \\ 6.0 & 4.823-22) & 2.936(-22) & -39.1 \\ 7.0 & 4.854(-22) & 2.715(-22) & -44.1 \\ 8.0 & 4.888(-22) & 2.493(-22) & -49.0\end{array}$

$30 \mathrm{~cm}$ concrete shield

$\begin{array}{llll}1.0 & 1.264(-23) & 1.071(-23) & -15.3 \\ 2.0 & 3.497(-23) & 2.795(-23) & -20.1 \\ 3.0 & 5.046(-23) & 3.925(-23) & -22.2 \\ 4.0 & 6.067-23) & 4.479(-23) & -26.2 \\ 5.0 & 6.676(-23) & 4.656(-23) & -30.3 \\ 6.0 & 7.420(-23) & 4.616(-23) & -37.8 \\ 7.0 & 7.746(-23) & 4.462(-23) & -42.4 \\ 8.0 & 8.017(-23) & 4.248(-23) & -47.0\end{array}$

$*^{*} 4.204(-22)=4.204 \times 10^{-22}$ 
exposure that WALLGP computes. One obvious reason is pair production: MicroSkyshine includes annihilation photons, WALLGP does not. The uncertainty in WALLGP would be expected to increase as energy and the pair production cross-section increase. The effects of the "transparent wall" approximation made by MicroSkyshine are difficult to judge; the energy dependence of the dose due to photons which "pass through the wall" is not known. The third cause of disagreement, the deviation of MicroSkyshine's fitted response functions from the point-kernel data, may have an influence; the fits improve, however, as source energy increases, while the agreement of WALLGP and MicroSkyshine does not improve. The author feels that in this problem, WALLGP shows a serious deficiency in not including pair production. In its current form, it should be used with caution at higher source energies.

Another problem parameter which greatly affects dose from the source is the height of the wall between source and detector. WALLGP and Microskyshine were applied to a series of problems identical to those in Table 4-2, except that the source photons are restricted to $1 \mathrm{MeV}$, and the height of the wall above the source and detector is varied from $0.01 \mathrm{~mm}$ to $200 \mathrm{~m}$. The exposures per photon computed by MicroSkyshine and WALLGP are compared in Table 4-3. The source energy of $1 \mathrm{MeV}$ was chosen to eliminate errors in WALLGP from pair production. As would be expected, disagreement is largest for the highest walls because WALLGP does not include photons which scatter behind the wall. For the $200 \mathrm{~m}$ wall results, MicroSkyshine responses are nearly two orders of magnitude above those of WALLGP. Where the wall height is negligible, there are mucl smaller differences in the computed exposures; the disagreements are less than $15 \%$, smaller than some deviations of the fitted response functions in MicroSkyshine. The prominent cause 
Table 4-3. Comparison of detector responses computed by the codes MicroSkyshine and WALLGP with changes in wall height. Values of exposure per photon are presented for a point source of $1.0 \mathrm{MeV}$ photons placed $1 \mathrm{~m}$ behind a semi-infinite wall. The detector lies $399 \mathrm{~m}$ from the wall face opposite the source, and the source-detector axis is normal to the wall. The wall height equals the vertical distance from the top of the wall to either the source or detector. Air density is $1.25 \mathrm{mg} / \mathrm{cm}^{3}$; concrete density is $2.13 \mathrm{~g} / \mathrm{cm}^{3}$. All WALLGP results were $40 \mathrm{mfp}$.

\begin{tabular}{|c|c|c|c|}
\hline \multirow[b]{2}{*}{$\underset{\text { (in) }}{\text { Wall height }}$} & \multicolumn{2}{|c|}{ Normalized exposure ( $\mathrm{R} /$ photon) } & \\
\hline & $\begin{array}{l}\text { Computed by } \\
\text { MicroSkyshine }\end{array}$ & $\begin{array}{l}\text { Computed by } \\
\text { WALLGP }\end{array}$ & $\begin{array}{l}\text { Percent difference } \\
\text { from MicroSkyshine }\end{array}$ \\
\hline
\end{tabular}

No overhead shield

$\begin{array}{cccr}0.00001 & 2.861(-21) & 2.608(-21) & -8.8 \\ 0.01 & 2.736(-21) & 2.492(-21) & -8.9 \\ 0.1 & 1.977(-21) & 1.766-21) & -10.7 \\ 0.7 & 4.204(-22) & 3.606(-22) & -14.2 \\ 1.0 & 2.761(-22) & 2.313(-22) & -16.2 \\ 5.0 & 9.903(-23) & 6.840(-23) & -30.9 \\ 10 . & 8.754(-23) & 4.984(-23) & -43.1 \\ 50 . & 7.069(-23) & 1.341(-23) & -81.0 \\ 100 . & 6.603(-23) & 3.779(-24) & -94.3 \\ 200 . & 6.427(-23) & 3.456(-25) & -99.5\end{array}$

$30 \mathrm{~cm}$ concrete shield

$\begin{array}{cl}0.00001 & 1.344(-23) \\ 0.01 & 1.344(-23) \\ 0.1 & 1.344(-23) \\ 0.7 & 1.264(-23) \\ 1.0 & 1.087(-23) \\ 5.0 & 3.736(-24) \\ 10 . & 2.955(-24) \\ 50 . & 2.531(-24) \\ 100 . & 2.250(-24) \\ 200 . & 2.080(-24)\end{array}$

$1.157(-23)$

$-13.9$

$-13.9$

$-14.1$

$1.155(-23)$

$1.071(-23)$

$9.062(-24)$

$2.987\}-24$

$2.142(-24)$

$7.714(-25)$

$2.675(-25)$

$2.951(-26)$

$-15.3$

$-16.6$

$-20.0$

$-27.5$

$-69.5$

$-88.1$

$-98.6$

$* 2.861(-21)=2.861 \times 10^{-21}$ 
of disagreement in these cases is the following approximation made by MicroSkyshine: gamma rays which undergo their first interaction behind the wall from the detector contribute to measured dose, and the contribution may be found by treating the interaction point as a source of scattered photons and ignoring shielding effects of the wall.

Finally, Table 4-4 presents the results of WALLGP and MicroSkyshine for 23 configurations, grouped according to the parameter which varies from the base value shown in the first line. The purpose of this compilation is to describe the behavior of the responses as each aspect of the situation changes. For instance, as the detector is moved parallel to the wall, $z_{\alpha}$ increases; the source-detector separation increases, and both codes compute decreasing absorbed dose rates, as one would expect. As $y_{s}$ or $y_{d}$ increases, the wall presents more shielding and decreases the solid angle of directions from the source or detector which are not blocked; both MicroSkyshine and WALLGP report decreasing absorbed doses as these quantities increase. Both programs also confirm that increasing overhead shield thickness substantially reduces dose, as one would expect after reviewing the results of the benchmark experiment.

The two codes disagree on the behavior of detector response with changes in source energy. As the energy of source gamma rays increases, MicroSkyshine predicts response will drop until the pair production effect becomes noticeable, then predicts an increase in dose as photon energy and $(\mu / \rho)_{\mathrm{pp}}$ increase. WALLGP, which considers only Compton scattering effects, computes responses which continuously decline with increasing source energy and a dropping Compton cross-section. Table 4-2 reveals similar patterns for a different geometry; in this case, as then, WALLGP is proven to be flawed at higher energies because it neglects 
Table 4-4. Parametric study of detector responses conputed by the codes MicroSkyshine and KALLGP. Values of air kerma are presented for changes in source energy, position of source and detector, thickness of the overhead concrete shield, and air density. In all cases, concrete density is taken as $2.35 \mathrm{~g} / \mathrm{cm}^{3}$. Percent differences are the difference of WALLGP values from MicroSkyshine results.

\begin{tabular}{|c|c|c|c|c|c|c|c|c|c|c|}
\hline \multirow{2}{*}{$\begin{array}{l}\mathrm{E} \\
(\mathrm{MeV})\end{array}$} & \multirow{2}{*}{$\begin{array}{l}x_{s} \\
(\mathrm{~m})\end{array}$} & \multirow{2}{*}{$\stackrel{x_{d}}{(m)}$} & \multirow{2}{*}{$\begin{array}{l}y_{s} \\
(m)\end{array}$} & \multirow{2}{*}{$y_{d}$} & \multirow{2}{*}{$\begin{array}{l}z_{d} \\
(m)\end{array}$} & \multirow{2}{*}{$\stackrel{t}{(m)}$} & \multirow{2}{*}{$\begin{array}{l}\rho_{\mathrm{ai}} \\
\left(\mathrm{g} / \mathrm{cm}^{3}\right)\end{array}$} & \multicolumn{2}{|c|}{$\begin{array}{l}\text { Normalized air kerma } \\
\text { (rad/photon) }\end{array}$} & \multirow{2}{*}{$\stackrel{7}{\mathrm{diff}}$} \\
\hline & & & & & & & & MicroSky. & FALLGP & \\
\hline 1.25 & 3 & 100 & 3 & 3 & 10 & 0.10 & 1.2 & $6.050(-21)^{*}$ & $3.3364(-21)$ & -44.9 \\
\hline $\begin{array}{l}1.25 \\
1.25 \\
1.25 \\
1.25\end{array}$ & $\begin{array}{l}3 \\
3 \\
3 \\
3\end{array}$ & $\begin{array}{l}100 \\
100 \\
100 \\
100\end{array}$ & $\begin{array}{l}3 \\
3 \\
3 \\
3\end{array}$ & $\begin{array}{l}3 \\
3 \\
3 \\
3\end{array}$ & $\begin{array}{r}0 \\
30 \\
100 \\
300\end{array}$ & $\begin{array}{l}0.10 \\
0.10 \\
0.10 \\
0.10\end{array}$ & $\begin{array}{l}1.2 \\
1.2 \\
1.2 \\
1.2\end{array}$ & $\begin{array}{l}6.081(-21) \\
5.813(-21) \\
3.931(-21) \\
5.945(-22)\end{array}$ & $\begin{array}{l}3.3531(-21) \\
3.2123(-21) \\
2.2402(-21) \\
4.1111(-22)\end{array}$ & $\begin{array}{l}-44.9 \\
-44.7 \\
-43.0 \\
-30.8\end{array}$ \\
\hline $\begin{array}{l}2.25 \\
5.25 \\
7.25\end{array}$ & $\begin{array}{l}3 \\
3 \\
3\end{array}$ & $\begin{array}{l}100 \\
100 \\
100\end{array}$ & $\begin{array}{l}3 \\
3 \\
3\end{array}$ & $\begin{array}{l}3 \\
3 \\
3\end{array}$ & $\begin{array}{l}10 \\
10 \\
10\end{array}$ & $\begin{array}{l}0.10 \\
0.10 \\
0.10\end{array}$ & $\begin{array}{l}1.2 \\
1.2 \\
1.2\end{array}$ & $\begin{array}{l}5.001(-21) \\
4.415\{-21 \\
4.635(-21)\end{array}$ & $\begin{array}{l}3.1514(-21) \\
2.1763(-21) \\
1.7481(-21)\end{array}$ & $\begin{array}{l}-37.0 \\
-30.7 \\
-62.3\end{array}$ \\
\hline $\begin{array}{l}1.25 \\
1.25\end{array}$ & $\begin{array}{l}3 \\
3\end{array}$ & $\begin{array}{l}100 \\
100\end{array}$ & $\begin{array}{r}1 \\
10\end{array}$ & $\begin{array}{l}3 \\
3\end{array}$ & $\begin{array}{l}10 \\
10\end{array}$ & $\begin{array}{l}0.10 \\
0.10\end{array}$ & $\begin{array}{l}1.2 \\
1.2\end{array}$ & $\begin{array}{l}9.737(-21) \\
3.178(-21)\end{array}$ & $\begin{array}{l}6.0801(-21) \\
1.4633(-21)\end{array}$ & $\begin{array}{l}-37.6 \\
-54.0\end{array}$ \\
\hline $\begin{array}{l}1.25 \\
1.25 \\
1.25\end{array}$ & $\begin{array}{l}3 \\
3 \\
3\end{array}$ & $\begin{array}{l}100 \\
100 \\
100\end{array}$ & $\begin{array}{l}3 \\
3 \\
3\end{array}$ & $\begin{array}{r}-1 \\
1 \\
10\end{array}$ & $\begin{array}{l}10 \\
10 \\
10\end{array}$ & $\begin{array}{l}0.10 \\
0.10 \\
0.10\end{array}$ & $\begin{array}{l}1.2 \\
1.2 \\
1.2\end{array}$ & $\begin{array}{l}6.416(-21) \\
6.230\{-21 \\
5.454(-21)\end{array}$ & $\begin{array}{l}3.4972(-21) \\
3.4226(-21) \\
3.0108(-21)\end{array}$ & $\begin{array}{l}-45.5 \\
-45.1 \\
-44.8\end{array}$ \\
\hline $\begin{array}{l}1.25 \\
1.25\end{array}$ & $\begin{array}{l}3 \\
3\end{array}$ & $\begin{array}{r}10 \\
1000\end{array}$ & $\begin{array}{l}3 \\
3\end{array}$ & $\begin{array}{l}3 \\
3\end{array}$ & $\begin{array}{l}10 \\
10\end{array}$ & $\begin{array}{l}0.10 \\
0.10\end{array}$ & $\begin{array}{l}1.2 \\
1.2\end{array}$ & $\begin{array}{l}4.853(-20) \\
1.969(-25)\end{array}$ & $\begin{array}{l}2.6189(-20) \\
1.8563(-25)\end{array}$ & $\begin{array}{l}-46.0 \\
-5.7\end{array}$ \\
\hline $\begin{array}{l}1.25 \\
1.25 \\
1.25\end{array}$ & $\begin{array}{r}1 \\
10 \\
100\end{array}$ & $\begin{array}{l}100 \\
100 \\
100\end{array}$ & $\begin{array}{l}3 \\
3 \\
3\end{array}$ & $\begin{array}{l}3 \\
3 \\
3\end{array}$ & $\begin{array}{l}10 \\
10 \\
10\end{array}$ & $\begin{array}{l}0.10 \\
0.10 \\
0.10\end{array}$ & $\begin{array}{l}1.2 \\
1.2 \\
1.2\end{array}$ & $\begin{array}{l}3.218(-21) \\
9.263(-21) \\
2.858(-21)\end{array}$ & $\begin{array}{l}1.7680(-21) \\
5.2014(-21) \\
1.7330(-21)\end{array}$ & $\begin{array}{l}-45.1 \\
-43.8 \\
-39.4\end{array}$ \\
\hline $\begin{array}{l}1.25 \\
1.25\end{array}$ & $\begin{array}{l}3 \\
3\end{array}$ & $\begin{array}{l}100 \\
100\end{array}$ & $\begin{array}{l}3 \\
3\end{array}$ & $\begin{array}{l}3 \\
3\end{array}$ & $\begin{array}{l}10 \\
10\end{array}$ & $\begin{array}{l}0.10 \\
0.10\end{array}$ & $\begin{array}{l}1.1 \\
1.3\end{array}$ & $\begin{array}{l}5.856(-21) \\
6.189(-21)\end{array}$ & $\begin{array}{l}3.2403(-21) \\
3.4152(-21)\end{array}$ & $\begin{array}{l}-44 . \bar{T} \\
-44.8\end{array}$ \\
\hline $\begin{array}{l}1.25 \\
1.25 \\
1.25\end{array}$ & $\begin{array}{l}3 \\
3 \\
3\end{array}$ & $\begin{array}{l}100 \\
100 \\
100\end{array}$ & $\begin{array}{l}3 \\
3 \\
3\end{array}$ & $\begin{array}{l}3 \\
3 \\
3\end{array}$ & $\begin{array}{l}10 \\
10 \\
10\end{array}$ & $\begin{array}{l}0.0 \\
0.01 \\
1.00\end{array}$ & $\begin{array}{l}1.2 \\
1.2 \\
1.2\end{array}$ & $\begin{array}{l}1.449(-20) \\
1.402(-20) \\
5.180(-26)\end{array}$ & $\begin{array}{l}8.4088(-21) \\
7.8479(-21 \\
3.6336(-26)\end{array}$ & $\begin{array}{l}-42.0 \\
-44.0 \\
-29.9\end{array}$ \\
\hline
\end{tabular}

$* 6.050(-21)=6.050 \times 10^{-21}$ 
pair production. MicroSkyshine is more likely to predict the true response in such situations, although it can overpredict because of the "transparent wall" approximation mentioned earlier.

Another pattern revealed by MicroSkyshine and WALLGP occurs as $x_{S}$ is changed, as the source is moved toward or away from the wall. The highest dose occurs when the source is located $10 \mathrm{~m}$ from the wall; moving the source to a position $3 \mathrm{~m}$ or $1 \mathrm{~m}$ from the wall reduces the dose, because the "shadow" cast by the wall in the direction of the detector has been enlarged. In the other direction, moving the source out to $100 \mathrm{~m}$ from the wall also reduces the dose, but by attenuation, not by the shielding effect of the wall. Both programs behave properly in this set of problems, in a manner which makes intuitive sense.

The behaviors of the results of the two programs are similar in the parametric comparisons, but the values themselves are significantly different. Typically, the MicroSkyshine results are one and one-half to two times the doses computed by WALLGP. For most of these cases, pair production is negligible; the lack of fit of the response functions used by MicroSkyshine is not enough to help explain the discrepancy. The wall height is small compared to the source-detector distance, yet the MicroSkyshine approximation of scatters behind the wall could be a cause of disagreement. There may be other factors contributing to the difference, also; the point-kernel calculations which produced the MicroSkyshine response functions may differ markedly from the calculations performed by WALLGP, not as assumed.

In summary, both MicroSkyshine and WALLGP are likely to be inaccurate in estimating detector responses to a point source of gamma rays behind a wall. MicroSkyshine computes dose from photons which scatter behind the wall from the detector, neglecting the shielding effects of the wall and applying buildup to a direct 
path from the scattering volume to the detector. While this approach in a silo simulation compensates for photons which reflect from the interior walls of a silo as their first scatter, it may not be appropriate in the wall geometry. Gamma rays which reflect off the wall in a real situation will be directed away from the detector and should contribute little to measured dose. WALLGP should not be trusted in cases involving photon sources of moderate or high energies, for it neglects pair production, and annihilation photons are substantial for situations involving high-energy photons, such as ${ }^{16} \mathrm{~N}$ sources. If a wall in a real situation does not absorb source photons well and the MicroSkyshine approximation is appropriate, WALLGP will underestimate still further, since it only considers dose from photons scattering in full view of the detector. 


\subsection{CONCLUSIONS AND SUGGESTIONS FOR FURTHER STUDY}

The two goals of this work center on the two computer codes written for gamma-ray skyshine analysis. The first objective was to test modifications made to the point-kernel code of Roseberry, which computes the exposure or dose from a point gamma-ray source placed within a collimating silo. The second goal was to validate a similar code written for the ideal problem of a point gamma-ray source placed behind a semi-infinite wall. While SILOGP and WALLGP are based on the same point-kernel skyshine model, SILOGP appears to be much more reliable in the cases studied and agreed more closely with silo geometry reference values and other skyshine codes. Benchmark experimental data for the wall problem are not available, so that a definitive comparison cannot be made for WALLGP as it was for SILOGP. Yet, deficiencies are obvious in the code for the wall problem.

The first code, SILOGP, involved three major changes from Roseberry's code SKY, along with an approximation intended to correct for a non-ideal experimental condition. A Gauss quadrature routine and updated photon interaction data were slight improvements over SKY; the geometric-progression buildup factors which were introduced made a noticeable change in results, and are widely regarded as more accurate. To account for photons which can scatter within the confines of the silo before leaving it, the upper limit of integration over the supplement of the scattering angle was altered to simulate such photons in the dose computations. SKY $Y$ did nothing to imitate this interior scattering.

In comparisons to the benchmark experiment, this revised point-kernel code gave results with accuracy comparable to other numerical methods tested. Its best performance is for those geometries involving wide angles of collimation, where the 
source is relatively near the top of the silo. The ANSI Reference Problem and the $2 \pi$ problem of Faw and Shultis (Fa87) were examples of this, and proved that the model upon which the code is based is sound in this extreme case. Close to the source silo, the results of the code SILOGP should not be relied on, since it regularly underpredicts there. The approximation made to simulate scattering within the silo did not correct this problem entirely; the new geometric progression buildup factors may make the underprediction worse than it was with SKY. Farther from the source silo, SILOGP overpredicts, but within the range of distances considered, the difference is not extreme, and a conservative approach would demand overprediction rather than underprediction. In the presence of an overhead concrete shield, SILOGP produces more reliable results than many codes, because of the buildup approximation in concrete first made by Roseberry.

One improvement which can yet be made to this code is the inclusion of pair production interactions. While SILOGP did not show a tendency to underpredict where high-energy source photons were involved, the pair-production effect should be added for completeness, and the process can be added to the code easily. A second possibility suggested by Roseberry (Ros0) is that new buildup factors be computed for anisotropic sources, or for detectors at or near an interface between the air and the ground. All buildup factors currently available are for isotropic point sources and point detectors in infinite media only. While Shultis and Faw (Sh87) claim that adapted buildup factors are not necessary, such data would be well suited for this application and might reduce the overprediction of SILOGP at large distances. The computations required are beyond the scope of this work, however, and not a priority. 
WALLGP yields very reliable results in problems involving very low walls, such as the ANSI Standard Problem. As with SILOGP, the agreement of WALLGP with reference values validates the underlying theoretical model in the limiting case of $2 \pi$ geometry. There appears to be no tendency to overpredict or underpredict with distance, as there is with SILOGP; the results for sources shielded by overhead concrete are not seriously off, as they tend to be with $\mathrm{G}^{3}$. Still, with no experimental results available for comparisons, no definitive conclusions can be drawn for the problems WALLGP is intended for: a point source of gamma rays separated from detectors by a wall of shielding material of non-negligible height.

One difficulty in WALLGP made evident in this work involves the numerical integration of the exposure equation. The code encounters problems when the source and detector are relatively close together; it was discovered that increasing the mean free path cutoff criterion will lower the resulting estimate of detector response significantly. SILOGP shows no such difficulty, al though it uses the same quadrature subroutine. The probable cause of this is the cylindrical coordinate system chosen for WALLGP; SILOGP uses a different coordinate system, based entirely on angles. Although the two codes integrate the same exposure rate equation, the different coordinate systems require terms to be placed in a different order, and the expressions integrated by SILOGP and WALLGP take on different forms.

Figure 5-1 presents the values of the $\beta, \theta$, and $\epsilon$ integrands evaluated by SILOGP over the regions of integration, for a typical problem. Gauss-Legendre quadrature can easily integrate over these functions, and the adaptive quadrature used by SILOGP should simply refine the estimate of the areas under these curves. Figure $5-2$ presents the values of the $r, z$ and $\phi$ integrands evaluated by WALLGP 


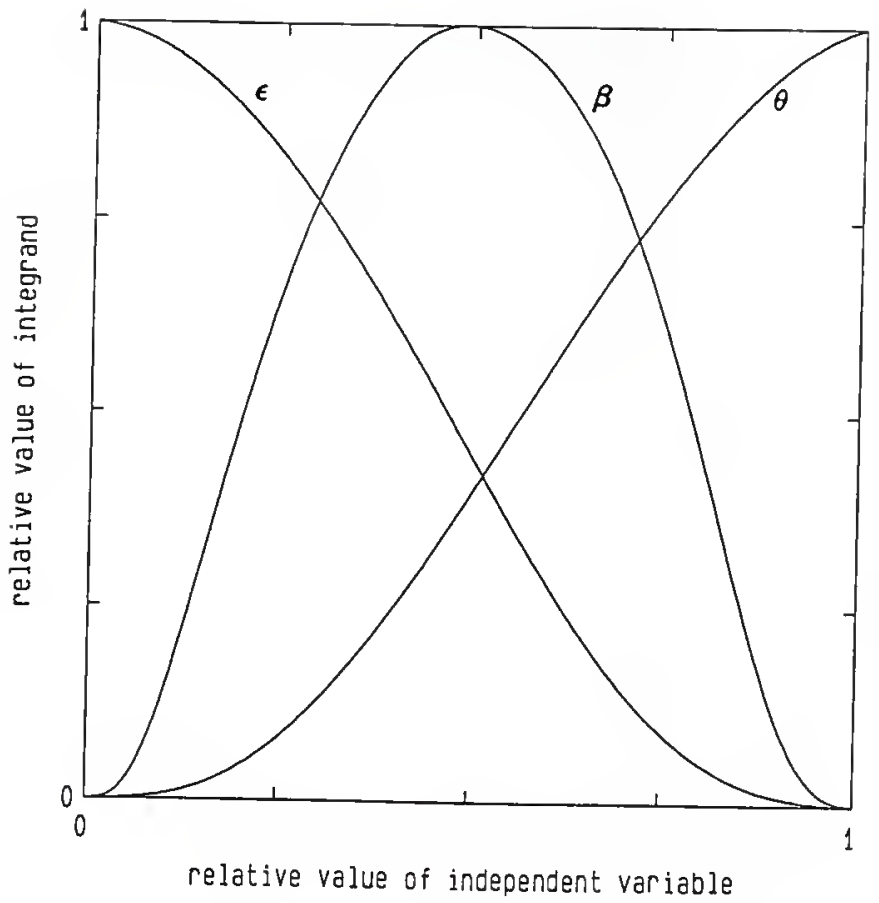

Fig. 5-1. Normalized graphs of the functions integrated by SILOGP. Each integrand is plotted for a single source-detector geometry. The abscissa of each integrand curve is normalized to a value of zero for the minimum value of $\beta, \theta$ or $\epsilon$, and a value of one for the maximum value of the variable. The value of the angle $\epsilon$, this intermalized in the same manner. The innermost integration is over the integrand, dependent is presented for a constant value of $\beta$ and $\theta$. The middle dependent only upon $\beta$. 


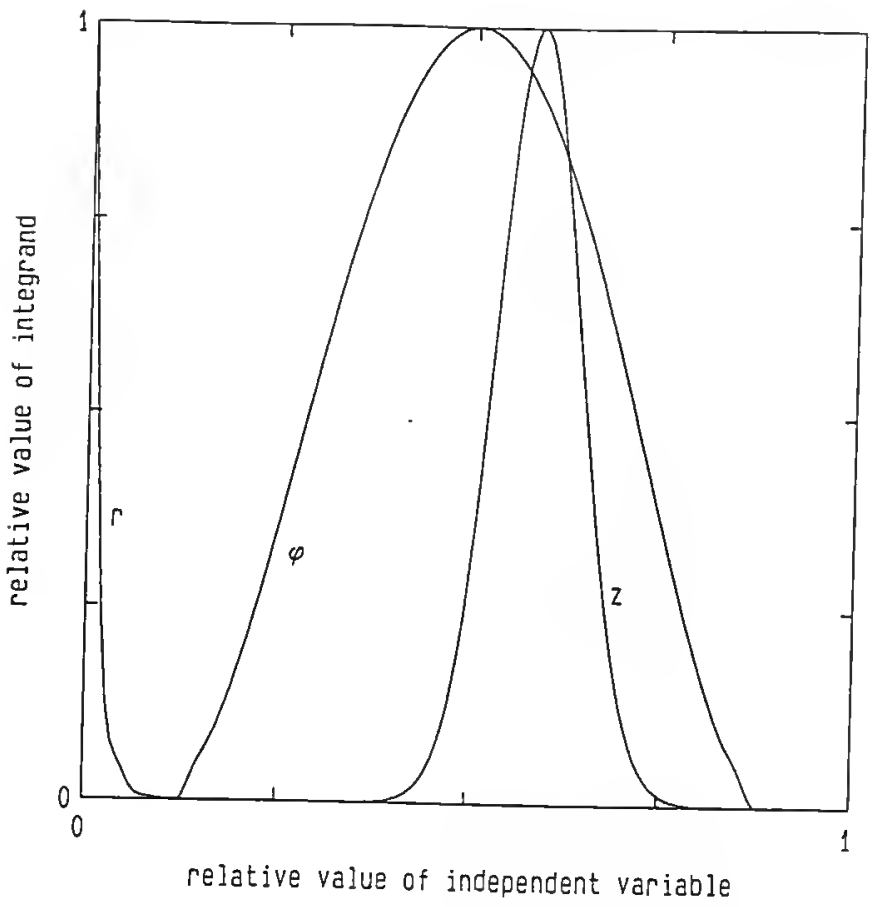

Fig. 5-2. Normalized graphs of the functions integrated by WALLGP. Each integrand is plotted for a single source-detector geometry. The abscissa of each integrand curve is normalized to a value of zero for the minimum value of $r, z$ or $\phi$, and a value of one for the maximum value of the variable. The value of the integrand is normalized in the same manner. The innermost integration is over the angle $\phi$; this integrand is presented for a constant value of $r$ and $z$. The middle integrand, dependent upon $z$, is graphed for constant $r$. The outer integrand is dependent only upon r. 
over their regions of integration. The r integrand asymptotically approaches zero quickly, and this outer integral could present problems for conventional Gauss-Legendre quadrature if the upper limit were extended too far. The adaptive Gauss quadrature in use here performs separate integrations over successively smaller sections of the entire region of integration until the change in the overall result is below the chosen level. It is conceivable that even this method would underestimate the integral; if the upper limit is extended too far, the large values of the integrand at low values of $r$ could be ignored or not accounted for properly.

Two approaches might be taken to alleviate this problem. The first is to abandon the cylindrical coordinate system in favor of the angular coordinate system used in SILOGP. The limits of integration for the wall geometry were developed first in the angular coordinate system, but proved to be very cumbersome and dependent on many variables in the source-detector geometry. Using the angular system may provide integrals like those in SILOGP, expressions easier to integrate using Gauss-Legendre quadrature. The second option is to complement the quadrature weights and zeros in the current program with weights and zeros from a different method. For instance, Gauss-Laguerre quadrature approximates a given function using Laguerre polynomials, which are variations of exponential functions (Sh88, Ho75). Ideally, the innermost integrals over $\phi$ and $z$ would be evaluated using weights and zeros from Legendre polynomials, then the outer $r$ integration would be performed with Laguerre weights and zeros. This may prove to be the more efficient option, if further study of the program is conducted.

The MicroSkyshine code provides most of the data for comparisons with WALLGP, and any weaknesses in MicroSkyshine, such as the errors in parametric fits to reference data, make judgments of the accuracy of WALLGP difficult. One 
obvious shortcoming of the present work pointed out by MicroSkyshine is the neglect of pair production from high-energy gamma rays. Although most of the codes used for comparisons did not compute dose from annihilation photons, MicroSkyshine results show that the decision to exclude them could ruin computed results in the event that pair production dominates photon interactions. The author strongly recommends that pair production be included in WALLGP for future work, to improve computed responses from sources of high-energy photons.

Data on the wall geometry are rare, and more studies of this problem may be beneficial. A benchmark experiment involving a gamma-ray source behind a wall would provide the best comparison by far, and could reveal other problems in WALLGP beyond those of pair production and the integration method. For example, it could show the importance of gamma rays which penetrate the shield wall, and perhaps validate the "transparent wall" approximation of MicroSkyshine. Because experiments of this type are rare and expensive, use of widely accepted numerical data will probably be necessary.

In summary, SILOGP and WALLGP should both be modified to include pair production, and the method of integration in the code for the wall problem should be reconsidered. This author feels that SILOGP is accurate and useful for the prediction of dose from a point gamma-ray source, but that WALLGP should not be relied upon, and that modifications to WALLGP and more conclusive comparisons should be performed before it is used in practical situations. 


\section{REFERENCES}

AN87 Material extracted from American National Standard ANSI/ANS-6.6.11987 with permission of the publisher, the American Nuclear Society. Ba88 Bassett, M. S., M.S. Thesis, Nuclear Engineering Department, Kansas State
University (1988).

Ch80 Chilton, A. B., C. M. Eisenhauer, and G. L. Simmons, "Photon Point Source Buildup Factors for Air, Water, and Iron," Nucl. Sci. Eng. 73, 97-107 (1980).

Ch84 Chilton, A. B., J. K. Shultis, and R. E. Faw, Principles of Radiation Shielding (Prentice-Hall, Inc., Englewood Cliffs, New Jersey, 1984).

Co69 Collins, D. G., F. O. Leopard, J. D. Marshall and M. B. Wells, Monte Carlo Procedures for Transport of $X$-rays and Fluorescent Light Through $a$ Spherical, Altitude-Dependent Atmosphere: Vol. III, Fluorescent Light Transport, Report RRA-T94-III, Radiation Research Associates, Fort
Worth, Texas, 1969.

Fa86 Faw, R. E., M. L. Roseberry, and J. K. Shultis, "Approximate Methods in Gamma-Ray Skyshine Calculations," in Approximate Calculational Techniques for Radiation Protection Applications, Report ORNL/RSIC-4S [ANS/SD-86/17], edited by A. F. Rice and R. W. Roussin (Oak Ridge National Laboratory, Oak Ridge, Tennessee, 1986), pp. 81-97.

Fa87 Faw, R. E., and J. K. Shultis, The MicroSkyshine Method for Gamma-Ray Skyshine Analysis, Kansas State University Engineering Experiment Station
Report 188, Manhattan, Kansas (1987). Ha83 Harima, Y., "An Approximation of Gamma-Ray Buildup Factors by
Modified Geometric Progression," Nucl. Sci. Eng. 83, 299-309 (1983).

Has6 Harima, Y., Y. Sakamoto, S. Tanaka, and M. Kawai, "Validity of the Geometric-Progression Formula in Approximating Gamma-Ray Buildup Factors," Nucl. Sci. Eng. 94, 24-35 (1986).

Ho75 Hornbeck, R. W., Numerical Methods (Quantum Publishers, Inc., New York,
New York, 1975).

Hu82 Hubbell, J. H., "Photon Mass Attenuation and Energy-absorption 1269-1290(1982). $1 \mathrm{keV}$ to $20 \mathrm{MeV}, "$ Int. J. Appl. Radiat. Isot. 33,

Ki68 Kitazume, M., "A Simple Calculation for Air-Scattered Gamma Rays," J.
Nucl. Sci. Technol. 5, 464-471 (1968).

La79 Lampley, C. M., The SKYSHINE-II Procedure: Calculation of the Effects of Structure Design on Neutron, Primary Gamma-Ray and Secondary Gamma-Ray Dose Rates in Air, Report RRA-T7901, Radiation Research
Associates, Fort Worth, Texas, 1979. 
Ly58 Lynch, R. E., J. W. Benoit, W. P. Johnson, and C. D. Zerby, A Monte Carlo Calculation of Air-scattered Gamma Rays, Report ORNL-2292, Oak Ridge
National Laboratory, Oak Ridge, Tennessee, 1958 .

Ma67 Malenfant, R. E., QAD: A Series of Point-Kernel General-Purpose Shielding Programs, Report LA-3573, Los Alamos Scientific Laboratory, Los Alamos, New Mexico, 1967.

Ma69 Marshall, J. D., M. B. Wells, F. O. Leopard and K. W. Tompkins, Monte Carlo Procedures for Transport of $X$-rays and Fluorescent Light Through a Spherical, Altitude-Dependent Atmosphere: Vol. I, X-ray Transport, Report RRA-T94-I, Radiation Research Associates, Fort Worth, Texas, 1969.

Ma73 Malenfant, R. E., G3: A General Purpose Gamma-Ray Scattering Program, Report LA-5176, Los Alamos Scientific Laboratory, Los Alamos, New Mexico, 1973.

Na79 Nason, R. R., "Analysis of Skyshine Spectral Measurements," M.S. Thesis, Nuclear Engineering Department, Kansas State University (1979).

Na81 Nason, R. R., J. K. Shultis, R. E. Faw, and C. E. Clifford, "A Benchmark Gamma-Ray Skyshine Experiment," Nucl. Sci. Eng. 79, 404-416 (1981).

Ro80 Roseberry, M. L., "Benchmark Skyshine Exposure Rates," M.S. Thesis, Nuclear Engineering Department, Kansas State University (1980).

Ro82 Roseberry, M. L., and J. K. Shultis, "Point Kernel Calculations of Skyshine Exposure Rates," Nucl. Sci. Eng. 80, 334-338 (1982).

RS85 Radiation Shielding Information Center, $G^{3}-6 E D$, General Purpose Gamma-Ray Scattering Code, Report CCC-75, Oak Ridge National Laboratory, Oak Ridge, Tennessee, 1985.

RS86 Radiation Shielding Information Center, Documentation for $C C C-493 / Q A D-C G G P$, Report CCC-493, Oak Ridge National Laboratory,
Oak Ridge, Tennessee, 1986 .

Sh87 Shultis, J. K., and R. E. Faw, Improved Response Functions For the MicroSkyshine Method, Kansas State University Engineering Experiment
Station Report 189, Manhattan, Kansas (1987).

Sh88 J. K. Shultis (private communication).

St67 Storm, E., and H. I. Israel, Photon Cross Sections from 0.001 to $100 \mathrm{MeV}$ for Elements 1 through 100, Report LA-3753, Los Alamos Scientific Laboratory,
Los Alamos, New Mexico, 1967.

Tr61 Trubey, D. K., "The Single-Scattering Approximation to the Solution of the Gamma-Ray Air-Scattering Problem," Nucl. Sci. Eng. 10, 102-116 (1961). 
We84 West, J. T., T. J. Hoffman, and M. B. Emmett, MORSE-SGC/S for the SCALE System, Report NUREG/CR-0200, Volume 2, Section F9 [ORNL/NUREG/CSD-2/V2/R2], Oak Ridge National Laboratory, Oak
Ridge, Tennessee, 1984.

Ze56 Zerby, C. D., Radiation Flux Transformation as a Function of Density of an Infinite Medium with Anisotropic Point Sources, Report ORNL-2100, Oak Ridge National Laboratory, Oak Ridge, Tennessee, 1956. 


\section{ACKNOWLEDGEMENTS}

The author wishes to thank Drs. Richard E. Faw and J. Kenneth Shultis of the Department of Nuclear Engineering, and Dr. Christopher Sorensen of the Department of Physics, for their insight and invaluable advice during this work. Thanks are also extended to Wendy Wittmer and Karen Demel of the KSU Engineering Experiment Station Drafting Room for their illustrations, and to Connie Schmidt for typing and patiently correcting the drafts and final version of this thesis.

The support of the Department of Nuclear Engineering during the course of this project is greatly appreciated. This research was performed under appointment to the Nuclear Engineering and Health Physics Fellowship program administered by Oak Ridge Associated Universities for the U.S. Department of Energy. 


\section{APPENDIX A}

The Computer Program SILOGP Developed in this Work for the Problem of a Point Gamma-Ray Source in a Cylindrical Silo

(Some subroutines required by this program are in Appendix C.) 
The progran SILOGP calculates the dose rate from a point isotropic gamma ray source collimated into a cone about the vertical and shielded by an overhead concrete slab.

C

The method is documented in the paper "Point Kernel Calculation of Skyshine Exposure Rates," Nucl. Sci. Engg., 80, 334-338 (1982), by M. L Roseberry and J. Kenneth Shultis. The program was written by M. L. Roseberry, (M.S. thesis, Kansas State University, 1980) and revised by R. E. Faw, 1985, and 0. L. George, 1987-88.

C

SILOGP integrates the singly scattered game-ray fluxes over a spatial region encompassing up to 40 mean free paths in air for the

$C$ total path length, including both air and a concrete shield. Inte-

C gration is performed by triple Gaussian quadrature, using an adaptive

C methad of integration. Interaction coefficients are taken from

C Hubbell, J. H., "Photon Mass Attenuation and Energy-Absorption

C Coefficients from $1 \mathrm{KeV}$ to $20 \mathrm{MeV}, "$ Int. J. Appl. Radiat.

C Isot., 33, 1269-1290, 1982. Gamma-ray exposure buildup

C factors are evaluated using the geonetric progression fitting

C function and data as reported in Harima, et al., "Validity of

C the Geometric Progression Gamna-Ray Buildup Factors," Nucl.

C Sci. Eng. 94, Sept. 1986. $\log -\log$ interpolation is used for

$C$ buildup factors and interaction coefficients. Conversion factors

C for prescribed dose equivalents are taken from standard

C ANSI/ANS-6.6.1-1977.

C

c The upper limit on source energy is $10 \mathrm{MeV}$. An error state arises if energies of scattered photons fall below $0.02 \mathrm{MeV}$.

In version 2.6, all geometry data is received from input files. The first record of the file lists source energy, angle of collimation, concrete thickness, and concrete and air densities. The remainder of the file consists of an unformatted list of source-detector distances (m). Oata output is via the console and a named output

$c$ file. Integration is performed with an error tolerance of $2 \%$, and cutoff values for distances in air and/or concrete are $40 \mathrm{mfp}$.

Results are given as the dose rate per unit source strength, multiplied by the square of the source-detector distance (m) and divided by the specified full-angle of collimation (sr).

The following subprograms must be linked for execution:

SILOGP THE MAIN PROGRAM

RESG GAMMA RAY RESPONSE FUNCTIONS

GMUHUB GAMMA RAY INTERACTIOM COEFFICIENTS 
179

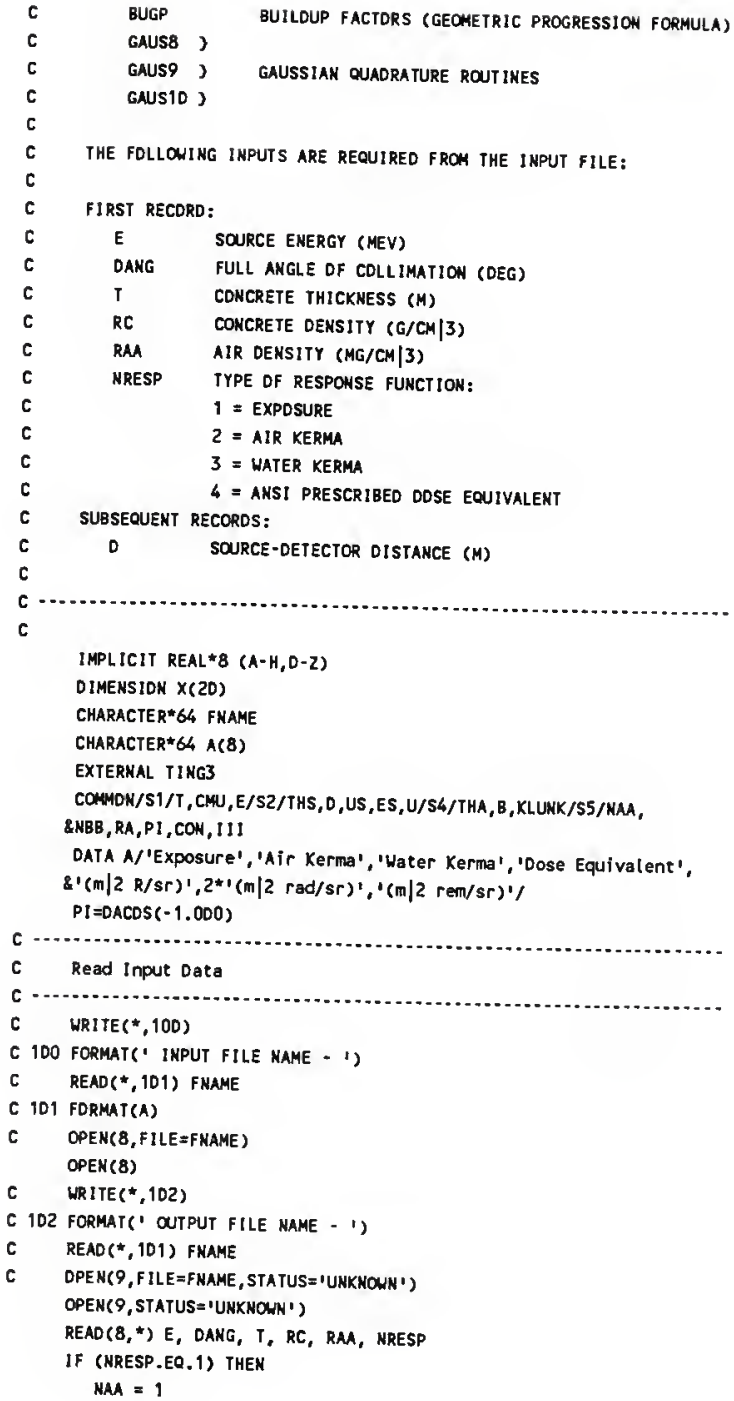




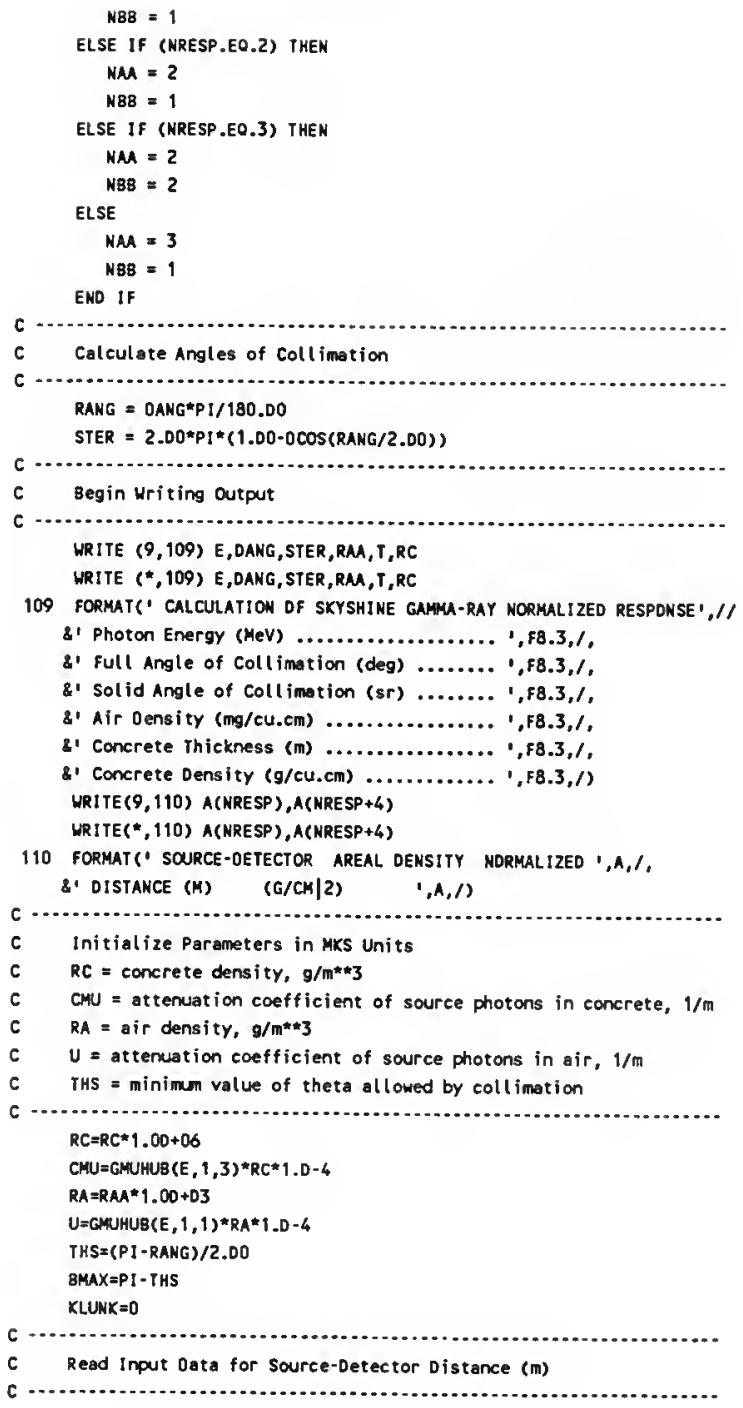


$20 \operatorname{READ}(8, *, E N D=11) 0$

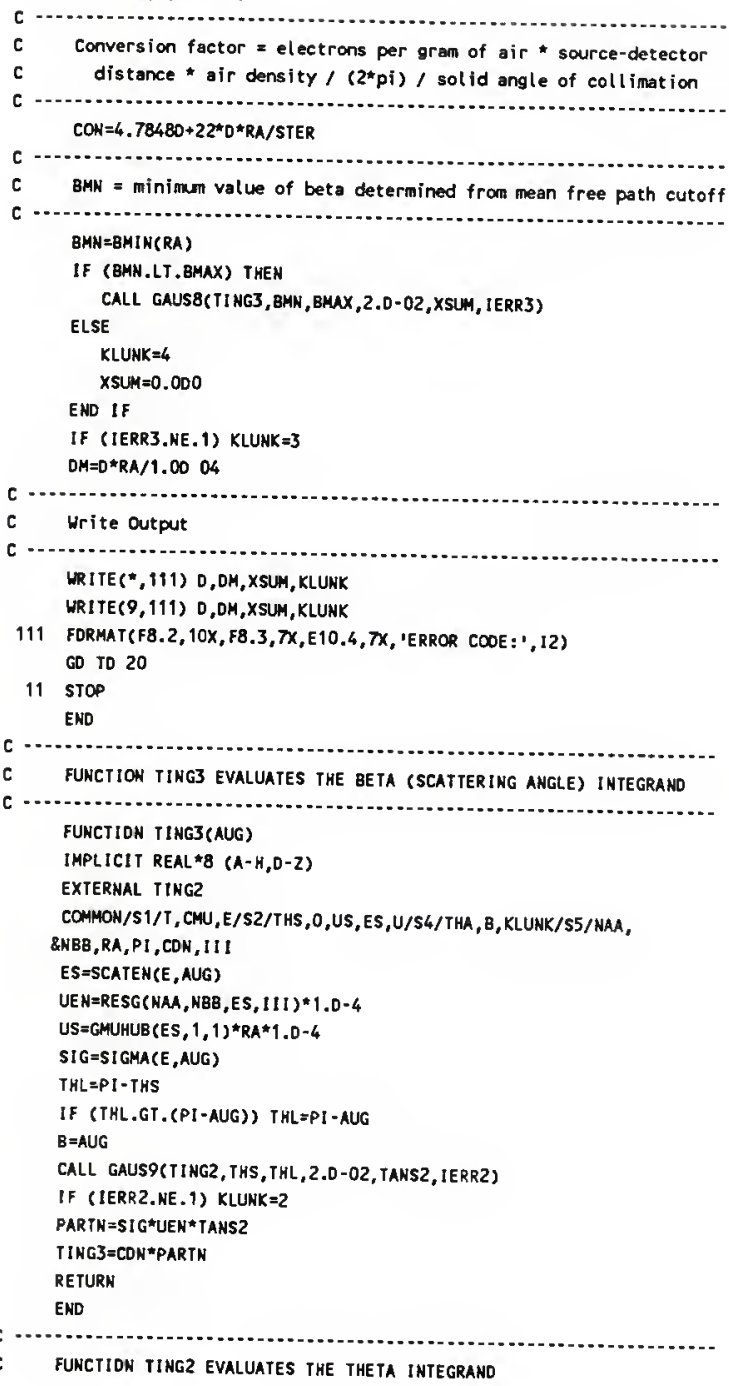




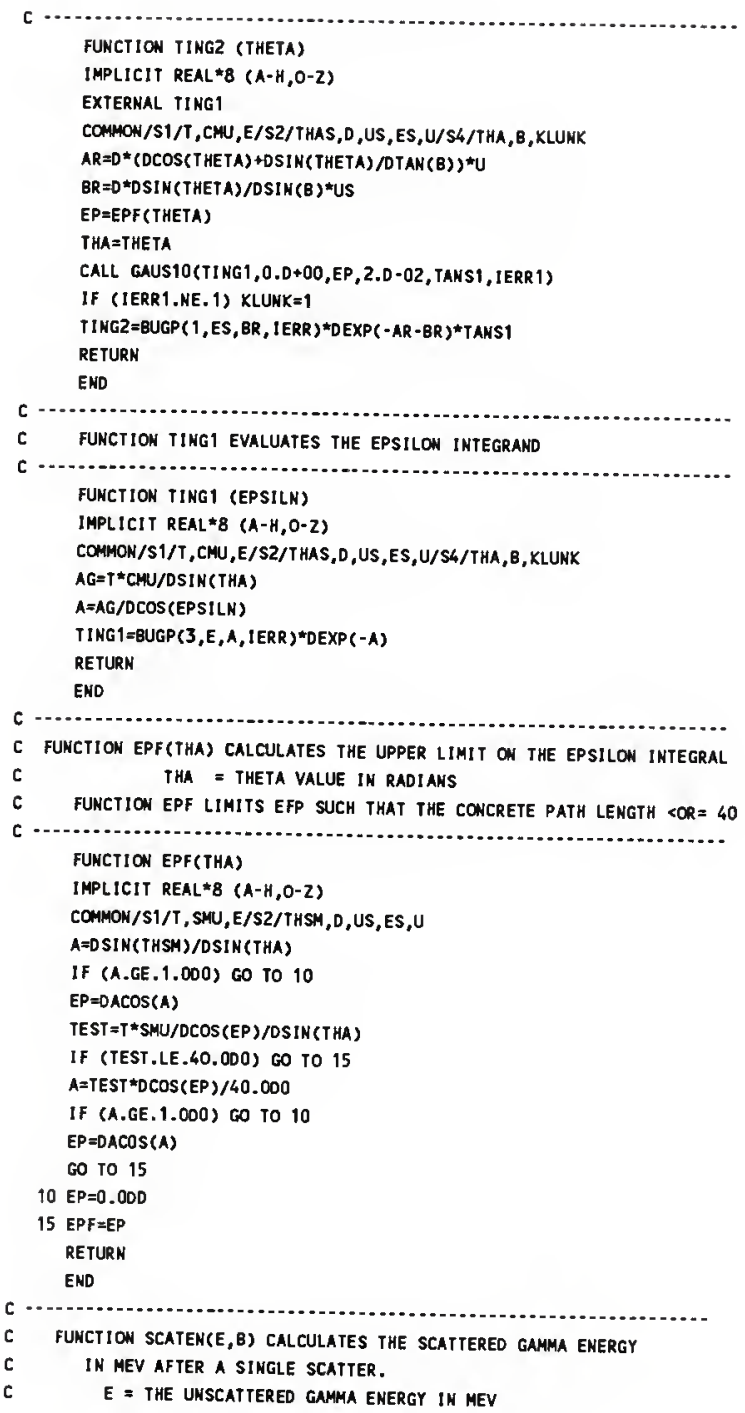




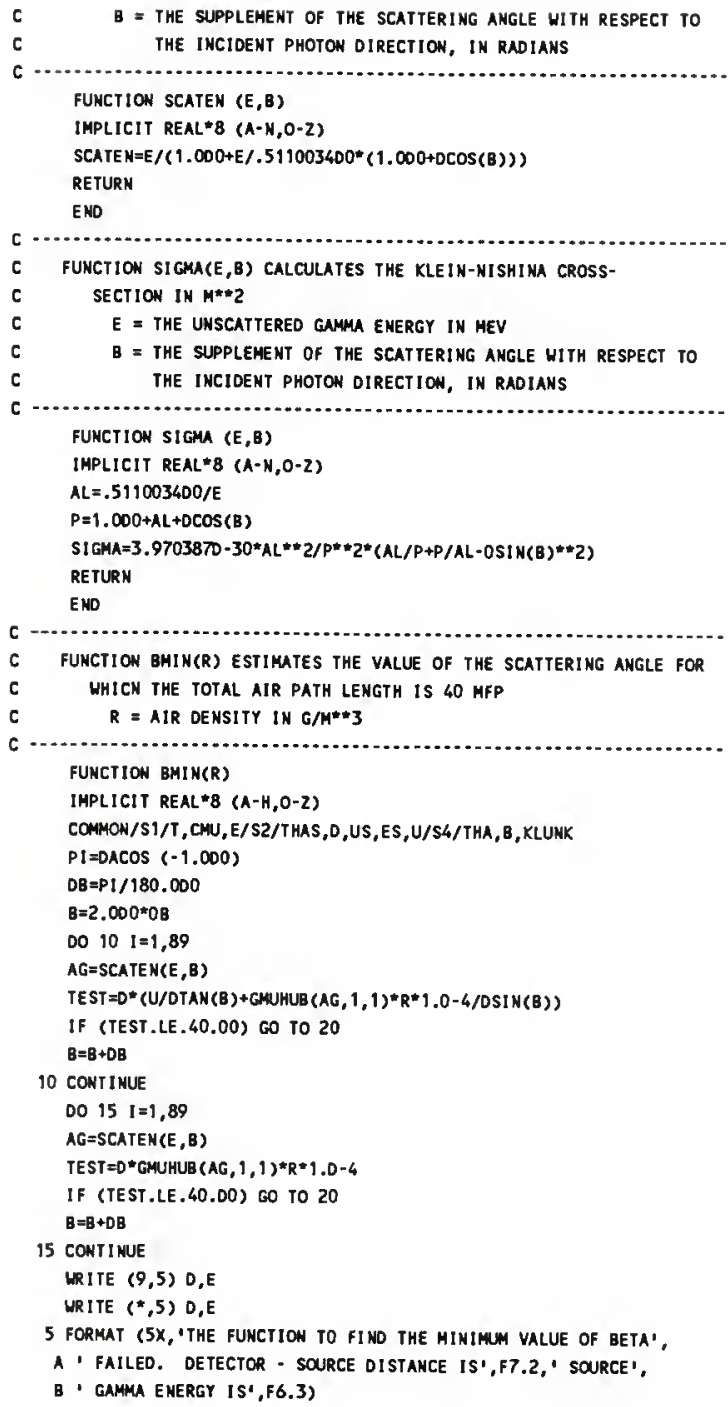


184

20 BMIN=B-DB

RETURN

END 


\section{APPENDIX B}

The Computer Program WALLGP Developed in this Work for the Problem of a Point Gamma-Ray Source Behind a Semi-Infinite Wall

(Some subroutines required by this program are in Appendix C.) 


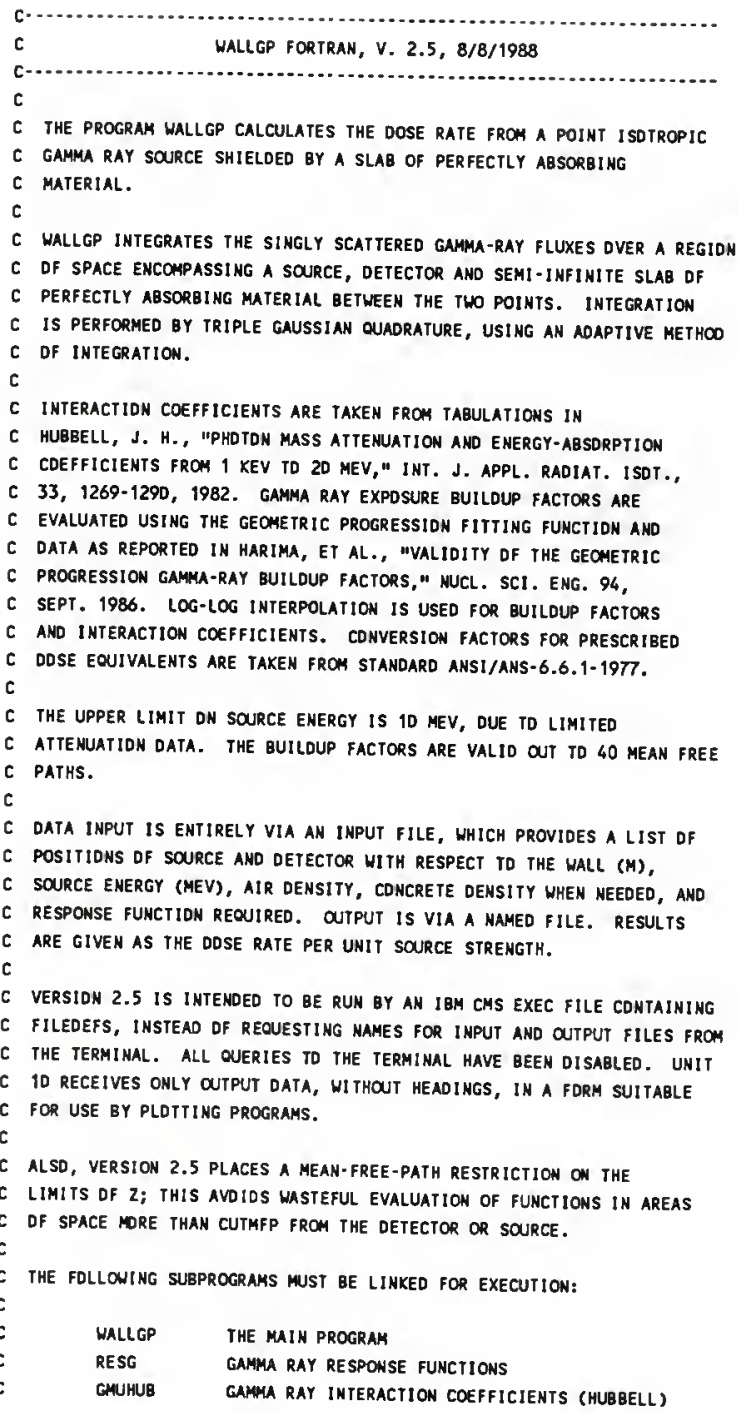


187

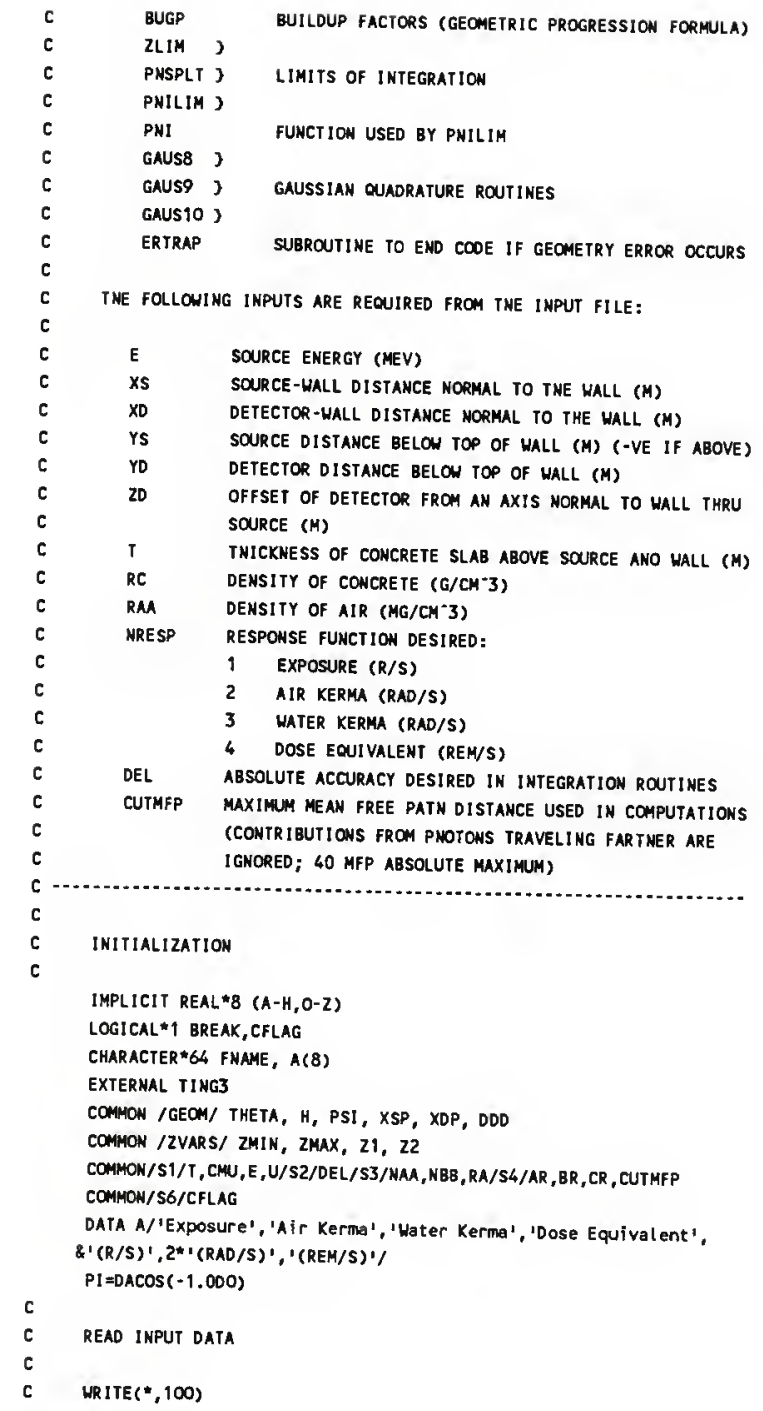




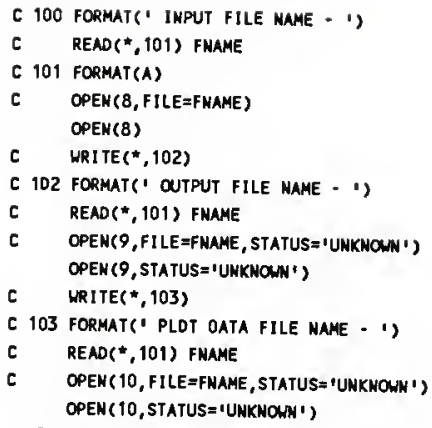


IF (E.GT.10.) THEN

WRITE $(9,207)$

WRITE $(*, 207)$

207 FORMAT (" ***INPUT ENERGY DF ',F8.2,' MEV IS TOO LARGE***') GO TO 20

ELSE IF (XS.LE. $(0.0+0) . O R . X D . L E .(0.0+0))$ THEN

WRITE $(9,202)$

URITE $(*, 202)$

202 FORMAT('**\#IMPROPER GECMETRY: SOURCE AND DETECTDR DN SAME SIDE DF

\& HAL $(* * * 1)$ GO TD 20

ELSE IF (YS.LT.(O.DO).AND.T.GT.(O.DO)) THEN

WRITE $(9,205)$

URITE (*.205)

205 FORMAT ( $1 * *$ IMPROPER GEOMETRY: SOURCE IS ABDVE BDTTON DF CDNCRETE \&SLAB***1) GO TD 20

ELSE IF (T.GT. $(0.00)$. AND.RC.EO. $(0.00)$ ) THEM WRITE $(9,210)$

WRITE $(*, 210)$

210 FORMAT(' ***CONCRETE SHIELD NDT ASSIGNED A DENSITY***1) GO TD 20

ELSE IF (CUTMFP.GT.40.) THEN

WRITE $(9,206)$

WRITE $(*, 206)$

206 FDRMAT ('***CUTDFF VALUE DF ',F8.2, ' MFP IS TOO LARGE***1) GO TD 20

ELSE

END IF

C

C CONVERT TD CYLINDRICAL COORDINATE SYSTEM

C $\quad H=$ height of wall edge above source-detector axis = minimum $r$

C THETA = angle of rotation between $X$ and $X^{\prime}$ axes

C PSI = angle of rotation between $X^{\prime}$ and $X^{\prime \prime}$ axes

C $X S P=$ distance in $X I$ direction from source to top of wall

c $X D P=$ distance in $X+$ direction from detector to top of wall

C $\quad$ DDD = distance separating source and detector

c RMAX = maximum value of $r$

c

THETA $=$ DATAN(CYS - YD) $/(X S+X D))$

$H=Y S$ * DCOS(THETA) - XS * DSIH(THETA)

IF (H.LE. $(0 . D+0)$ ) THEH

WRITE $(9,203)$

WRITE $(*, 203)$

203 FDRMAT(" ***IMPROPER GEOMETRY: SQURCE AHD DETECTOR DH A CLEAR LIME

\& DF SIGHT***1)

GO TD 20

ELSE

END IF

$X S P=X S * D C O S(T H E T A) * Y S * D S I N(T H E T A)$ 


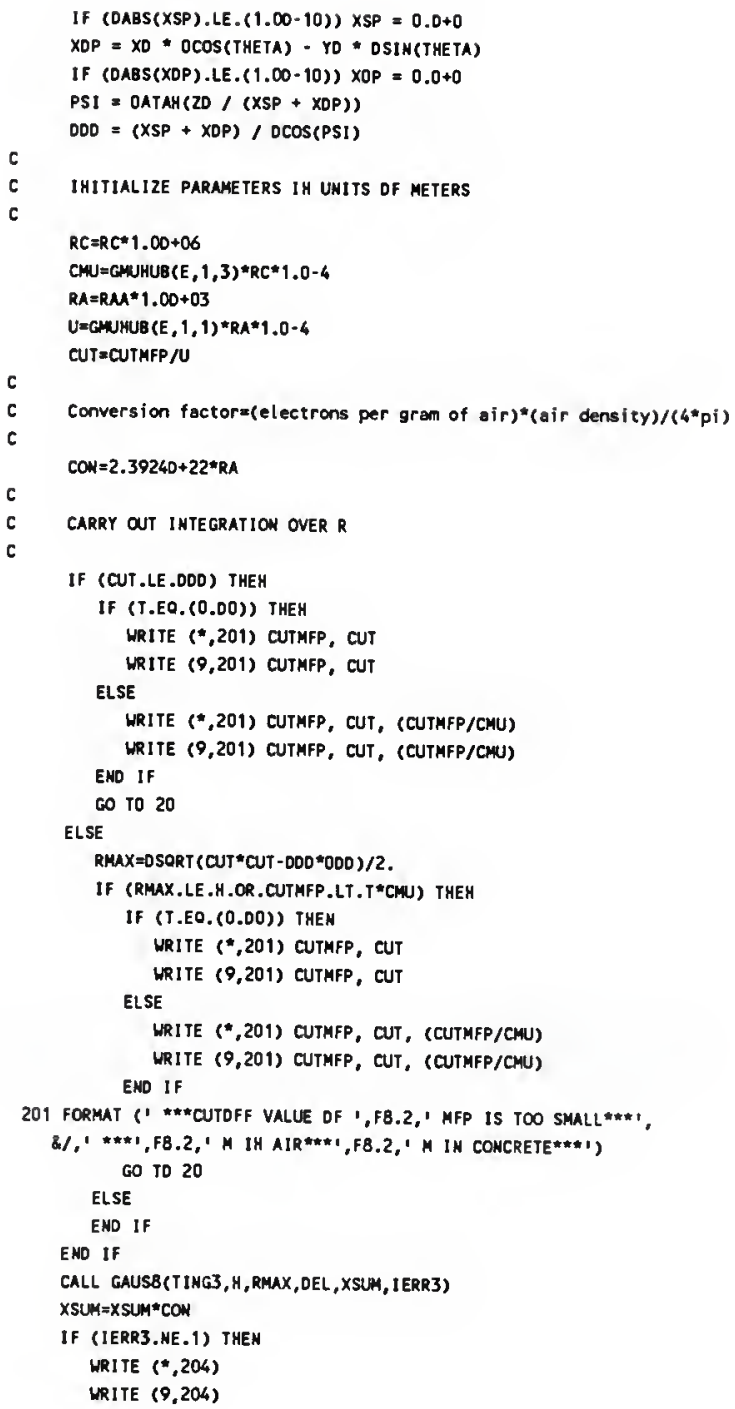


204 FORMAT( ' ***INACCURATE ESTIMATE OF R INTEGRAL***1)

ELSE

END IF

c

c WRITE OUTPUT

c

IF (XSUM.EO. $(0.0+00)$.AND.CFLAG) THEN

URITE $(*, 208)$ CUTMFP

WRITE $(9,208)$ CUTMFP

208 FORMATS' ***CUTOFF VALUE OF $1,78.2$, MFP IS TOO SMALL***1,1,1***A

\&LL. EVALUATEd PATH LENGTHS IN CONCRETE EXCEed CUTDFF VALUE*\#*1)

ELSE

WRITE $(9,112)$ A (NRESP)

WRITE $(*, 112)$ A (NRESP)

112 FORMAT(' Normalized ',A20, 'Response rate * $d^{\star \star 2}$ ')

WR 1 TE $(*, 113) \times S U M, A(N R E S P+4), \times S U *$ *ODD*DDD,A(NRE SP +4)

WRITE $(9,113) \times$ XSUM, A(NRESP +4$), \times S U M * D O O * D D D, A(N R E S P+4)$

113 FORMAT ( $1 X, 1$ PE 10.4, 1X,A7, 13X, 1PE10.4, 1X, 1 $m * \star 2 * 1, A 7)$

WRITE $(10,114)$ ODD ,DDD*RAA/10. XSUM, XSUM*DDD*DDD

114 FORMAT(2(F7.2,2X), 2(1PE10.4,1X))

END IF

CO TO 20

11 STOP

END

C

FUnCtION TING3 EVALUATES the R INTEgRano

C $\quad R=$ value of $r$ at which GAUSB evaluates the integrand

C RMAX = upper limit of integration over $r$

C $\quad 21, z 2=z$ coordinates at which the wall edge is a distance $r$ from

c the source-detector axis. Limits on phi will merge or change

c expressions at these values of $z$.

C TERM = discriminant of the quadratic whose solutions are $\mathbf{z 1}$ and

C $\quad 22$

C ZMIN, ZMAX = limits of integration over $z$

C ZMINL, ZMAXL = limits on $z$ computed from the source-detector

C geometry; compared to limits computed from the mean free path

C cutoff eriterion to determine ZMIN and ZMAX

c

DOUBLE PRECISION FUNCTION TING3(R)

IMPLICIT REAL*8 (A-H,O-Z)

EXTERNAL TING2

COMMON /GEOM/ THETA, H, PSI, XSP, XDP, DDD

COMMON /ZVARS/ ZMINL, ZMAXL, Z1, ZZ

COMMON /ERVARS/ RPT, ZPT

COMMON/S1/T,CMU,E,U/S2/DEL/S4/AR, BR, CR, CUTMFP

RP $T=R$

CUT=CUTMFP $/ U$

RMAX $=D S O R T(C U T \star C U T-D O D \star D D D) / 2$.

c

C COMPUTE $Z$ BREAKPOINTS WHERE PHI WILL SWITCH EXPRESSIONS 
c

TERM $=0 S I N(P S I) * D O R T(R * R-N * N)$

$21=(X S P-T E R M) / D \operatorname{COS}(P S T)$

$22=(X S P+T E R H) / D C O S(P S I)$

c

C CARRY OUT INTEGRATION OVER $Z$

c

CALL ZLIM(R, 21, 22, ZMINL, ZMAXL)

ZMIN=DMAX1 (ZMINL, (DDO-CUT*DSORT (1.-R*R/RMAX/RMAX)) /2.)

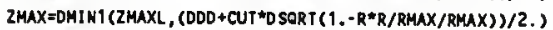

CALL GAUS9 (TING2, ZMIN, ZMAX,DEL, ZANS, IERR2)

IF (IERR2.NE.1) THEN

WRITE $(9,200)$

WRITE $(*, 200)$

200 FORMAT( 1 **INACCURATE ESTIMATE OF Z INTEGRAL***)

ELSE

END IF

TING3 $=R *$ ZANS

RETURN

ENO

C

C FUNCIION IINGZ EVALUATES THE $Z$ INTEGRAND

C $\quad z=$ value of $z$ at wich the integrand ia evaluated

C BETA = supplement of the photon acattering angle

C $\quad M A=$ distance $(m)$ traveled by photon in air before scattering

C $\quad A R=$ mean free paths traveled by photon in air before scattering

$C \quad B B B=$ distance $(m)$ traveled by photon in air after scattering

C $\quad B R=$ mean free paths traveled by photon in air after scattering

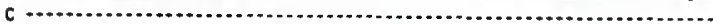

OOUBLE PRECISION FUNCTION IING2 (2)

IMPLICIT REAL\#8 (A-N,O-2)

LOGICAL*I BREAK, CFLAG

EXTERHAL TINGI

COMHON /GEOM/ THETA, N, PSI, XSP, XDP, DDD

COMMON /ZVARS/ ZMIN, ZMAX, 21, 22

COMMON /ERVARS/ R, 2PT

COMMON/S1/T, CMU, E, U/S2/DEL/S3/NMA, NBB, RA/S4/AR, BR, CR, CUTHFP

COMMON/SG/CFLAG

2PT $=2$

c

C COMPUTE SCATTERED GAMMA LEG LENGTH, B

c

BETA=DATAN $(Z / R)+D A T A N((D D D-Z) / R)$

ES=SCATEN(E, BETA)

UEN $=R E S G(N M, N B B, E S, 111) * 1 . D-6$

IF (111.NE.0) THEN

WRITE $(*, 300) 111$

WRITE $(9,300)$ III

300 FORMAT(" *\#ERROR ',11," IN COMPUTATION OF RESPONSE FUNCTION**1)

ELSE 
ENO IF

$S I G=S I G M A(E, B E T A)$

$B B B=0 S O R T(R * R+(000-2) *(000-2))$

US $=$ GMUHUB $(E S, 1,1) * R A * 1.0-4$

$B R=U S * B B B$

C

C

6

C

$c$

c

C

c

C

C

C

c

c

c

C

C

C

C

C

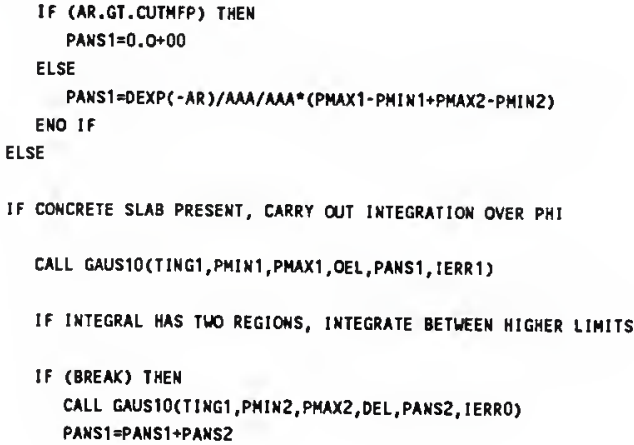




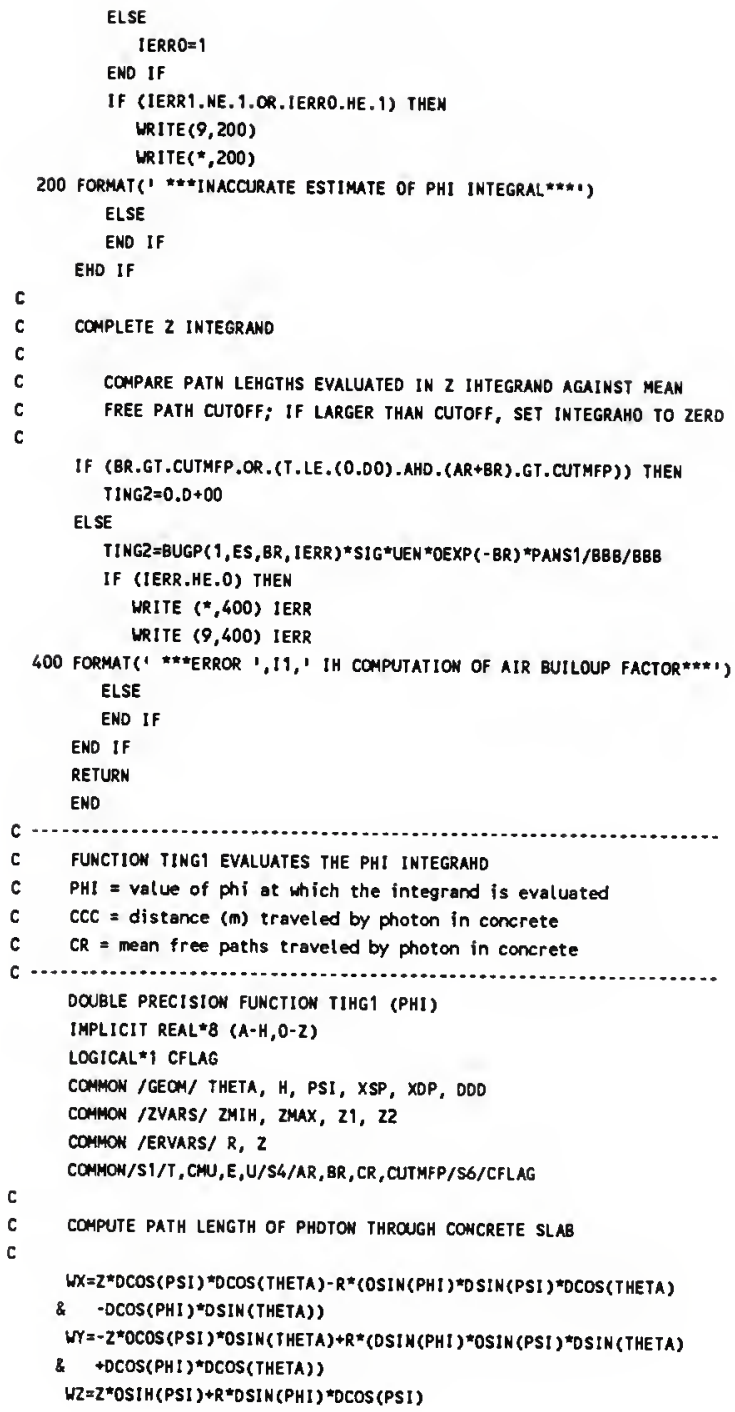




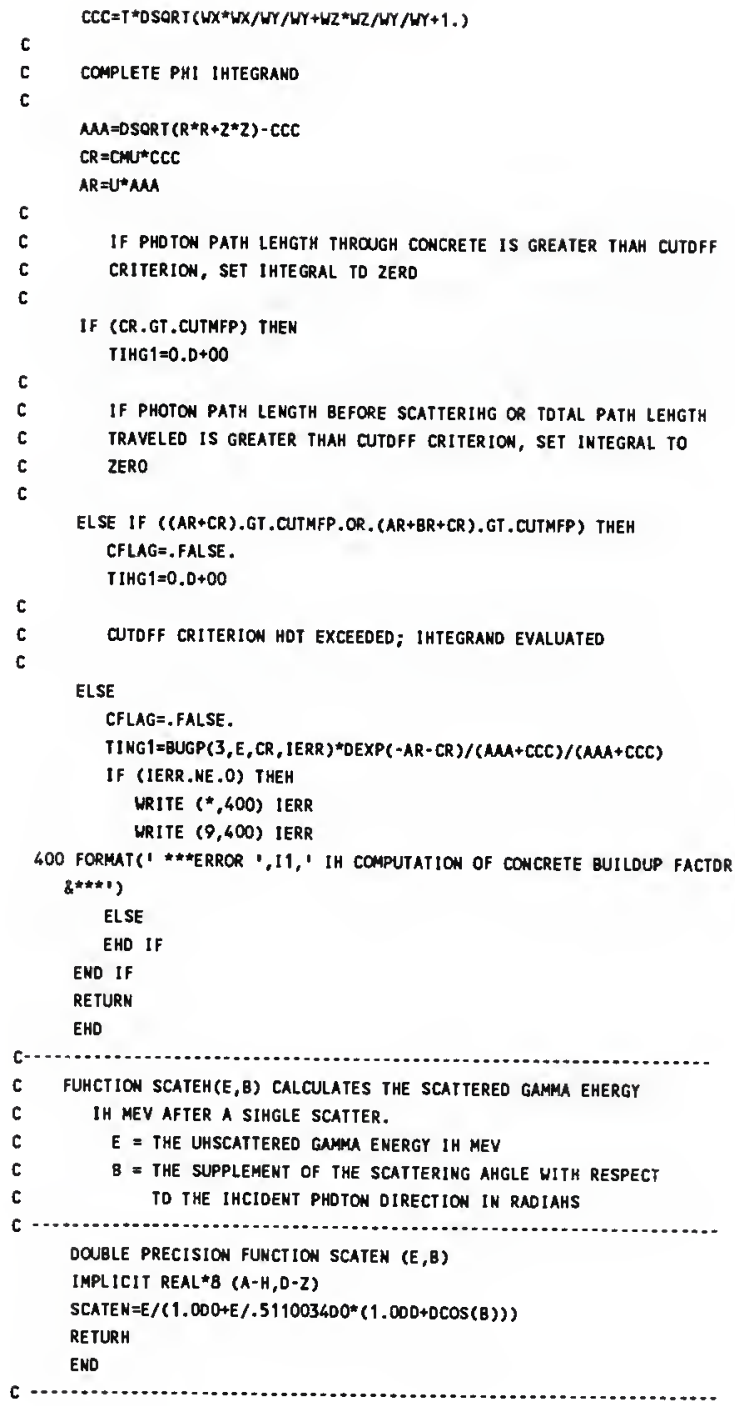


196

C FUHCIION SIGMA(E, B) CALCULATES THE KLEIH-MISHIHA CROSS.

C SECTION IH H**2

C E = THE UNSCATTERED GAMMA ENERGY IH MEV

C $B$ THE SUPPLEMEHT OF THE SCATTERIHG AHGLE WITH RESPECT

C TO THE INCIDEHY PHOTCN DIRECTION IH RADIANS

\section{C -}

DOUBLE PRECISION FUNCTION SICMA (E,8)

IMPLICIT REAL*8 (A-H,O-Z)

$A L=.511003400 / E$

$P=1.000+A L+D \cos (B)$

$\operatorname{SI~GMA=3.9703870-30*AL**2/P**2*(AL/P+P/AL-DSIN(B)**2)}$

REYURH

END 


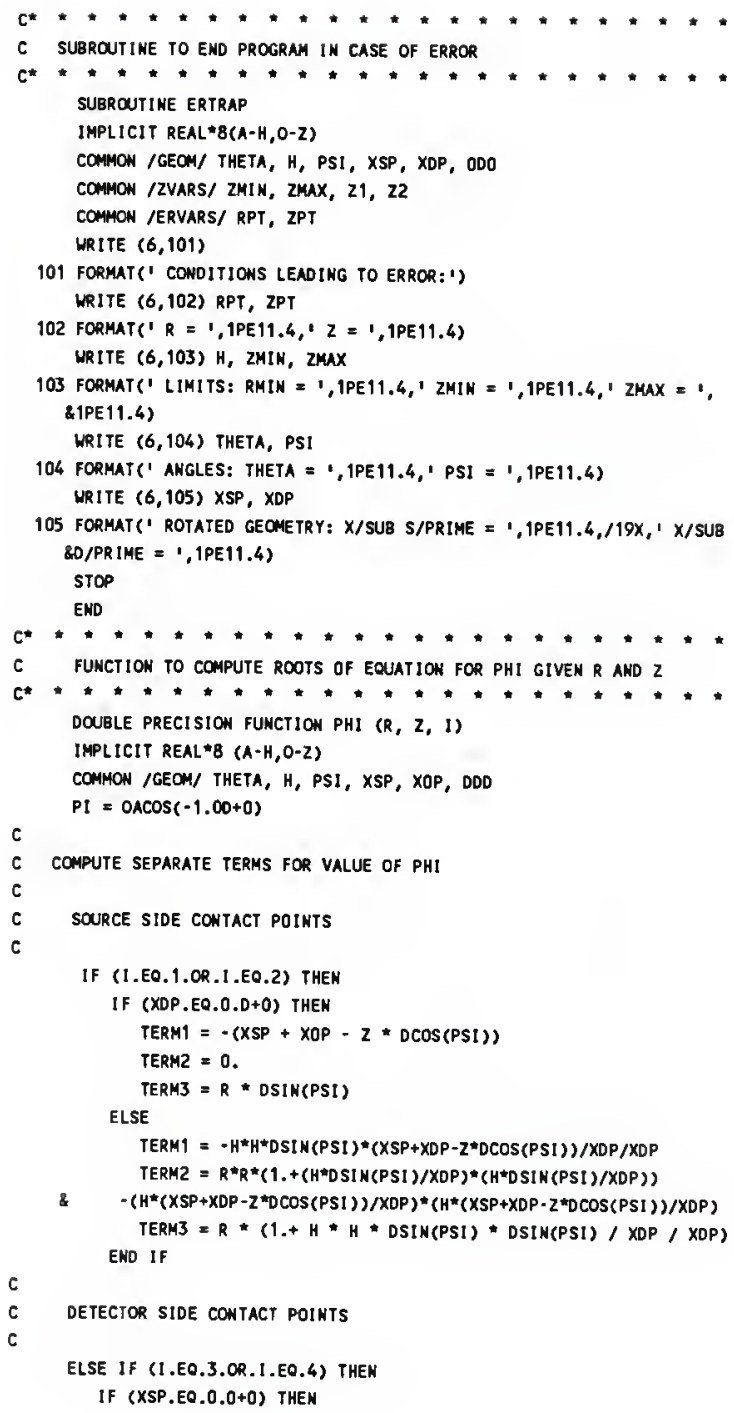




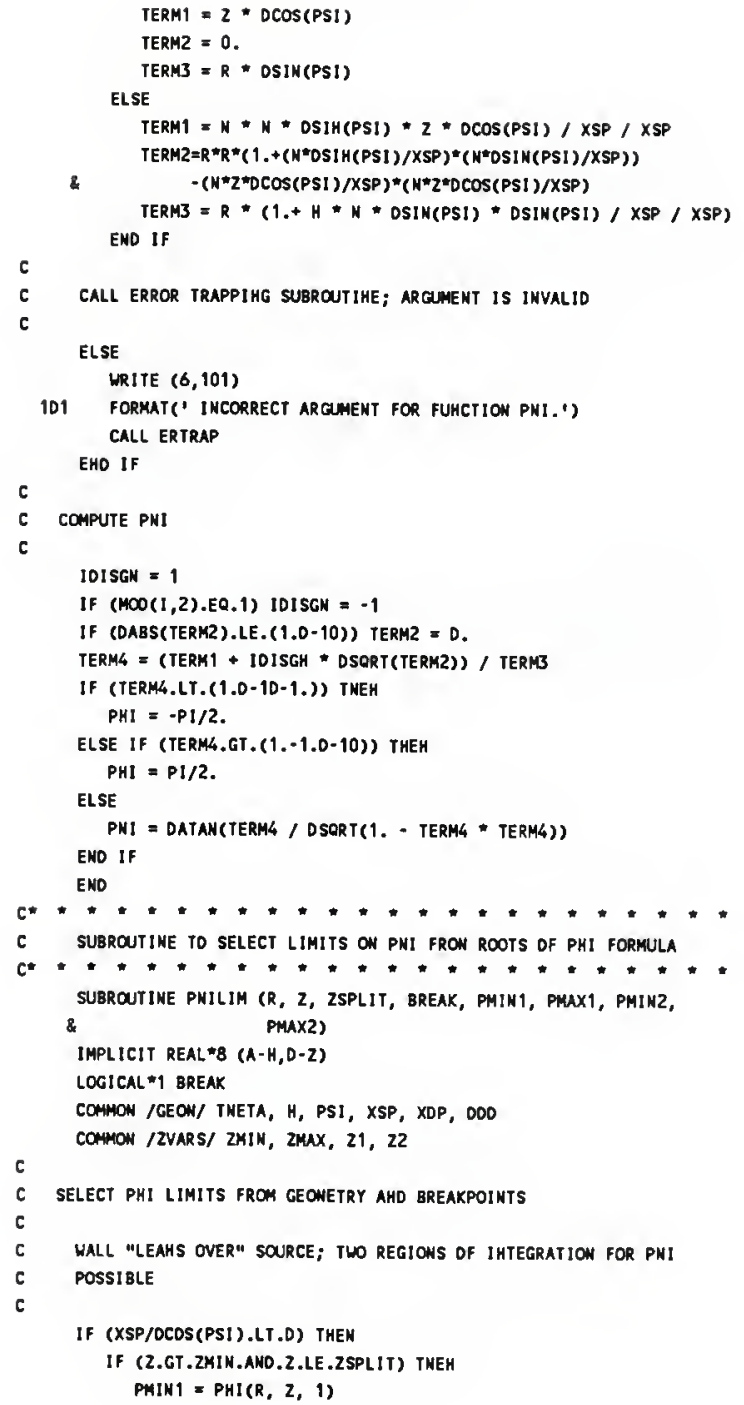




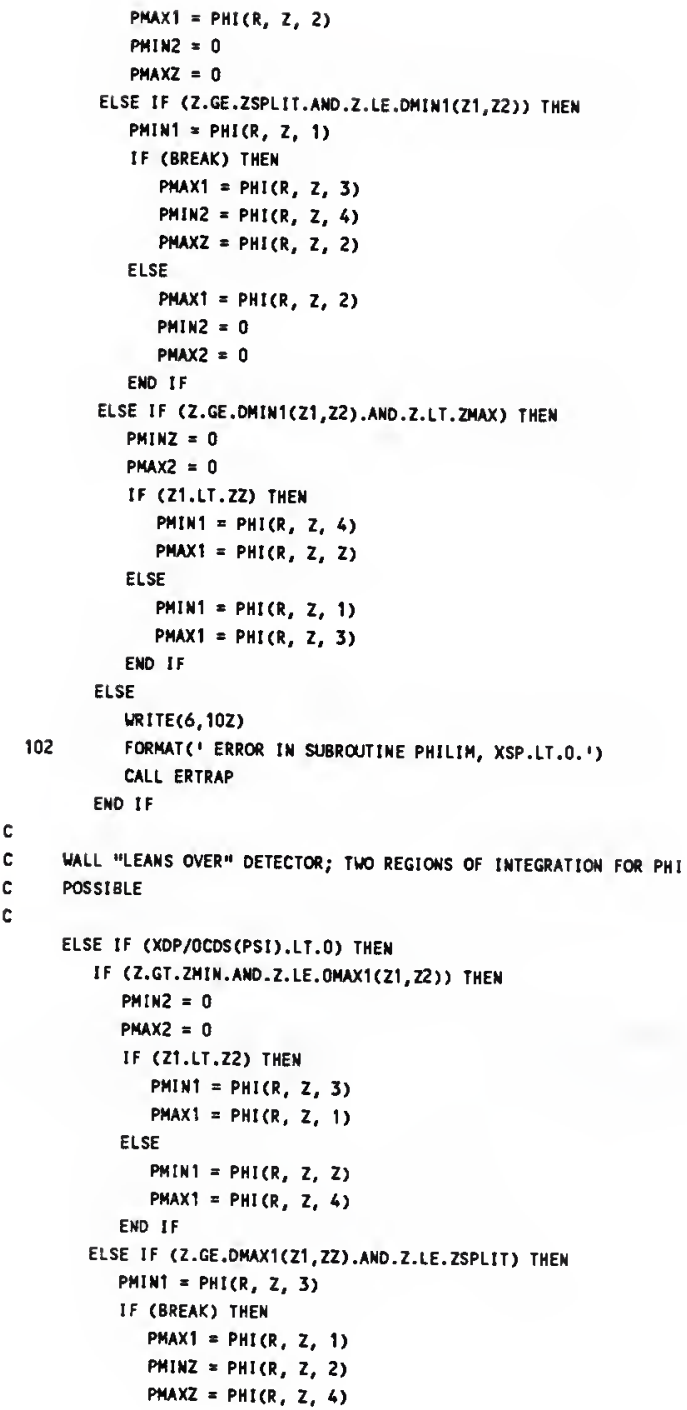




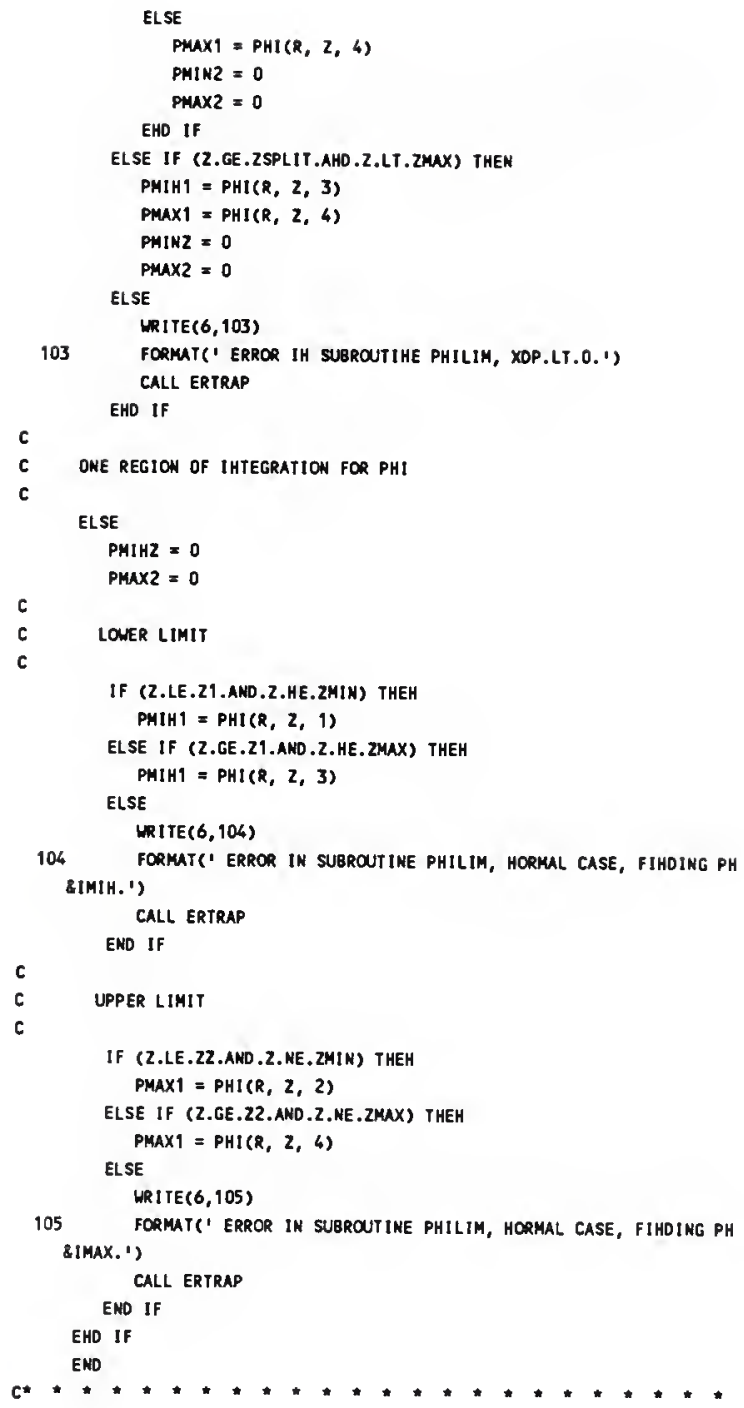




\section{1}

C SURROUTINE TO COMPUTE POSSIBLE BREAKPOINTS FOR PHI LIHITS

C*

SUBROUTINE PHSPLT (R, ZSPLIT, BREAK)

IMPLICIT REAL $\#$ \& (A-H,D-Z)

LOGICAL*1 BREAK

COMHOW /GEOM/ THETA, H, PSI, XSP, XDP, DOD

COMHON /ZVARS/ ZMIN, ZMAX, Z1, Z2

BREAK = .FALSE.

C

C SELECT GEOMETRIES WHERE SPLIT POSSIBLE

c

IF (R.GT.H.AND.(XSP.LT.D.CR.XDP.LT.D)) THEN

C

C FIND VALUES OF 2 UHERE INNER LIMITS APPEAR

C

IF (XSP.LT.D) THEN

ZSPLIT $=-D S E R T\left(\left(R^{\star X S P / H / D C O S}(P S I)\right) *(R * X S P / H / O C D S(P S I))\right.$

\& $\left.\quad *\left(1+H^{*} H^{*} D S I N(P S I) * D S I N(P S I) / X S P / X S P\right)\right)$

ELSE

ZSPLIT $=00 D+D$ SORT $\left(\left(R^{\star} X D P / H / D C O S(P S I)\right)^{*}\left(R^{*} X D P / H / O C O S(P S I)\right)^{*}\right.$

8 $\left(1+H^{*} H^{*} D S[N(P S I) * D S[N(P S I) / X D P / X D P))\right.$

END If

c

C CONFIRM THAT INHER LIMITS ARE ON SCATTERING ZONE AND COMPUTE THEM

c

IF (ZSPLIT.GE.ZMIN.AND.ZSPLIT.LE.ZMAX) THEN

IF (XSP.LT.O) THEN

PHIT = PHI (R, ZSPLIT, १)

PNI2 $=$ PHI $(R$, ZSPLIT, 3$)$

PHI3 = PHI (R, ZSPL1T, 4)

PHIL = PNI (R, ZSPLIT, 2)

ELSE

PHIT = PHI (R, ZSPLIT, 3)

PHI2 $=$ PHI $(R, Z$ ZPLIT, 1$)$

PHI3 = PHI (R, ZSPLIT, 2)

PHI4 = PHI (R, ZSPLIT, 4)

END IF

IF (PHI2.NE.PHI3) THEN

WR ITE 6,106$)$

106

FORMAT(' VARIABLE ZSPLIT HAS BEEN COMPUTED INCORRECTLY.' ) CALL ERTRAP

END IF

C

C FINO IF PHI REGION DF INTEGRATION DOES SPLIT (INMER LIMITS ARE

C INSIDE OUTER LIMITS)

c

IF (PHI1.LT.PHI2.AND.PHI3.LT.PHI4) THEN BREAK = .TRUE.

ELSE

END IF 


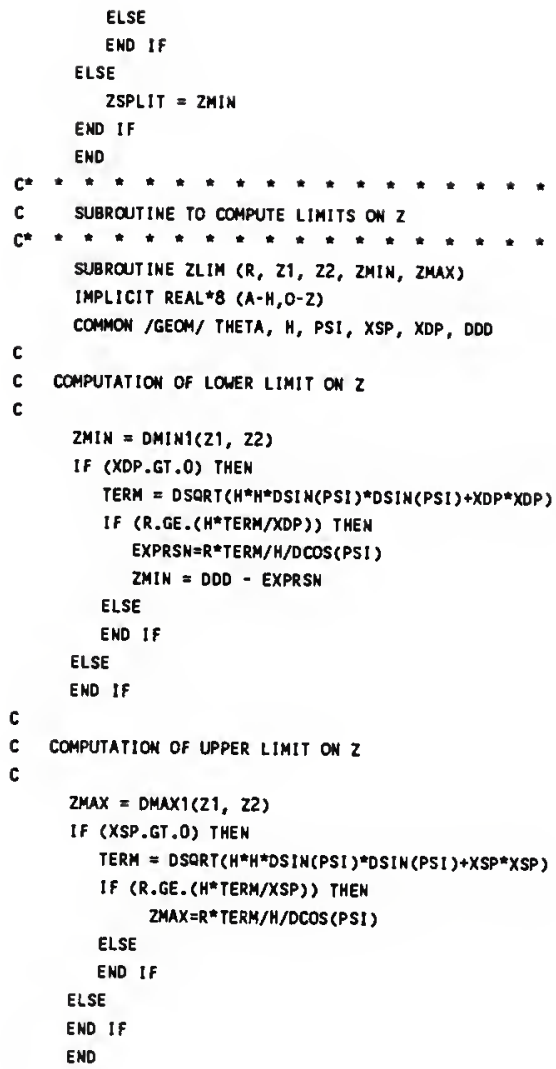




\section{APPENDIX C}

Subroutines Required by SILOGP and WALLGP 


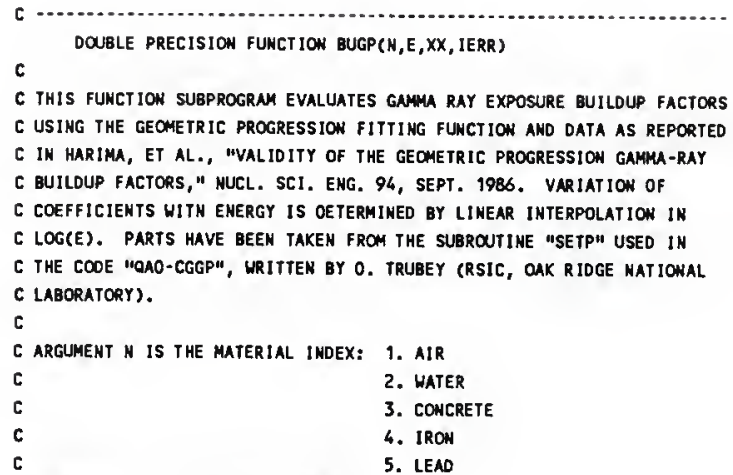




\section{5}

\& $2.212,1.544,-0.105,14.36,0.0437,2.103,1.441,-0.089,14.22,0.0378$, \& $1.939,1.269,-0.058,16.52,0.0246,1.839,1.173,-0.039,14.07,0.0161$, $1.710,1.056,-0.013,11.82,0.0047,1.621,0.989,0.004,13.45,-0.0041$, \& $1.554,0.939,0.018,13.55,-0.0122,1.507,0.903,0.029,16.13,-0.0272$, $1.422,0.879,0.035,13.36,-0.0191,1.362,0.859,0.042,13.37,-0.0247$, $1.267,0.043,0.047,15.08,-0.0336$

c

C data for concrete

OATA ( $(8 F S E T(3, J, K), K=1,5), J=1,25) / 1.029$,

\& $0.364,0.240,14.12,-0.1704,1.067,0.389,0.214,12.68,-0.1126$,

\& $1.212,0.421,0.201,14.12,-0.1079,1.455,0.493,0.171,14.53,-0.0925$,

\& $1.737,0.628,0.115,15.82,-0.0600,2.125,0.664,0.118,11.90,-0.0615$,

$\& 2.557,0.895,0.042,14.37,-0.0413,2.766,1.069,0.001,12.64,-0.0251$,

$\& 2.824,1.315,-0.049,8.68,-0.0048,2.716,1.430,-0.070,18.52,0.0108$,

$2.522,1.492,-0.082,16.59,0.0161,2.372,1.494,-0.085,15.96,0.0194$,

$2.271,1.466,-0.082,16.25,0.0195,2.192,1.434,-0.078,17.02,0.0199$,

\& $2.066,1.386,-0.073,15.07,0.0202,1.982,1.332,-0.065,15.38,0.0193$,

$1.848,1.227,-0.047,16.41,0.0160,1.775,1.154,-0.033,14.35,0.0100$, $\$ 1.671,1.054,-0.010,10.47,-0.0008,1.597,0.988,0.008,12.53,-0.0115$, $81.527,0.951,0.020,9.99,-0.0184,1.478,0.940,0.021,13.11,-0.0163$, \& $1.395,0.917,0.028,13.45,-0.0213,1.334,0.901,0.035,12.56,-0.0267$, \& $1.260,0.823,0.065,16.28,-0.0581 /$

c

C data for iron

OATA ( $($ BFSET $(4, J, K), K=1,5), J=1,25) / 1.004$.

$1.583,-0.565,5.53,0.3568,1.012,0.130,0.620,11.39,-0.6162$,

\& $1.028,0.374,0.190,29.34,-0.3170,1.058,0.336,0.248,11.65,-0.1188$, \& $1.099,0.366,0.232,14.01,-0.1354,1.148,0.405,0.208,14.17,-0.1142$, $1.267,0.470,0.180,14.48,-0.0974,1.389,0.557,0.144,14.11,-0.0791$, $81.660,0.743,0.079,14.12,-0.0476,1.839,0.911,0.034,13.23,-0.0334$, $1.973,1.095,-0.009,11.86,-0.0183,1.992,1.187,-0.027,10.72,-0.0140$, \& $1.974,1.230,-0.036,9.30,-0.0110,1.942,1.251,-0.041,7.89,-0.0090$, $81.892,1.244,-0.040,6.95,-0.0123,1.846,1.223,-0.037,6.74,-0.0131$, $1.750,1.197,-0.040,15.90,0.0110,1.712,1.126,-0.022,7.34,-0.0047$, $\$ 1.627,1.059,-0.005,11.99,-0.0132,1.553,1.026,0.005,12.93,-0.0191$, \& $1.483,1.009,0.012,13.12,-0.0258,1.442,0.980,0.023,13.37,-0.0355$, $1.354,0.974,0.029,13.65,-0.0424,1.297,0.949,0.042,13.97,-0.0561$, \& $1.194,1.048,-0.002,5.01,0.0584 /$

C

C data for lead

DATA $((B F S E T(5, J, K), K=1,5), J=1,25) / 0$,

\& $0 ., 0 ., 0 ., 0 ., 0 ., 0 ., 0 ., 0 ., 0 ., 1.006,0.230,0.442,12.61,-0.5099$, \& $1.013,0.302,0.331,10.34,-0.3011,1.024,0.289,0.289,12.38,-0.1453$, \& $1.029,0.423,0.179,17.00,-0.1217,1.058,0.357,0.238,12.96,-0.1230$, \& $2.165,1.323,0.079,12.13,-0.0731,1.520,0.337,0.019,9.05,-0.0108$, \& $1.201,0.271,0.171,5.00,0.0842,1.135,0.523,0.140,17.37,-0.0874$, $1.180,0.597,0.113,16.76,-0.0596,1.233,0.631,0.107,14.62,-0.0533$, $81.271,0.684,0.089,14.56,-0.0417,1.334,0.738,0.073,13.84,-0.0334$, \& $1.372,0.789,0.059,13.44,-0.0288,1.409,0.865,0.039,13.11,-0.0217$, \& $1.425,0.903,0.036,13.26,-0.0319,1.383,0.967,0.027,13.51,-0.0385$ 


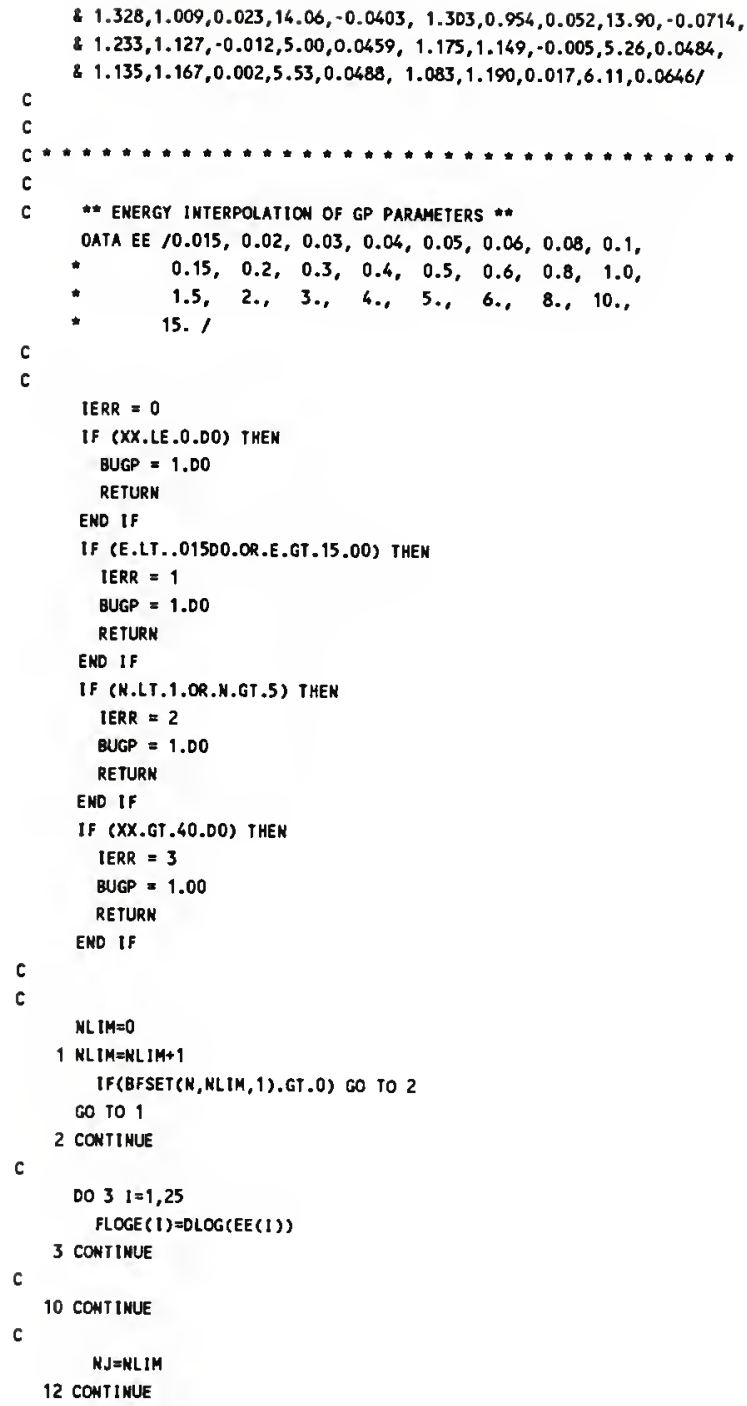




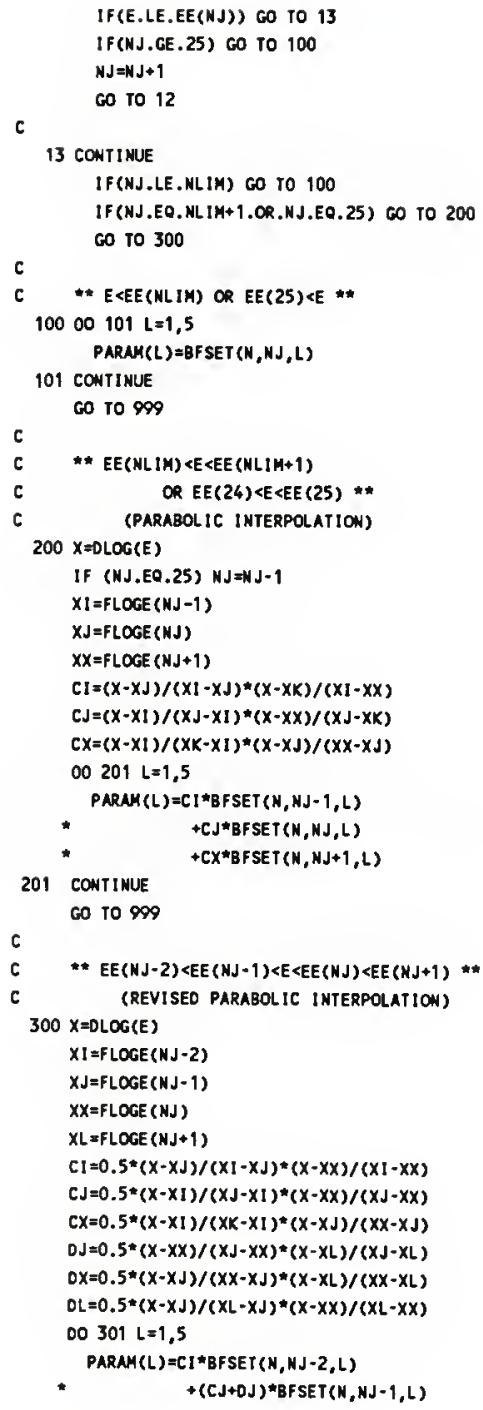




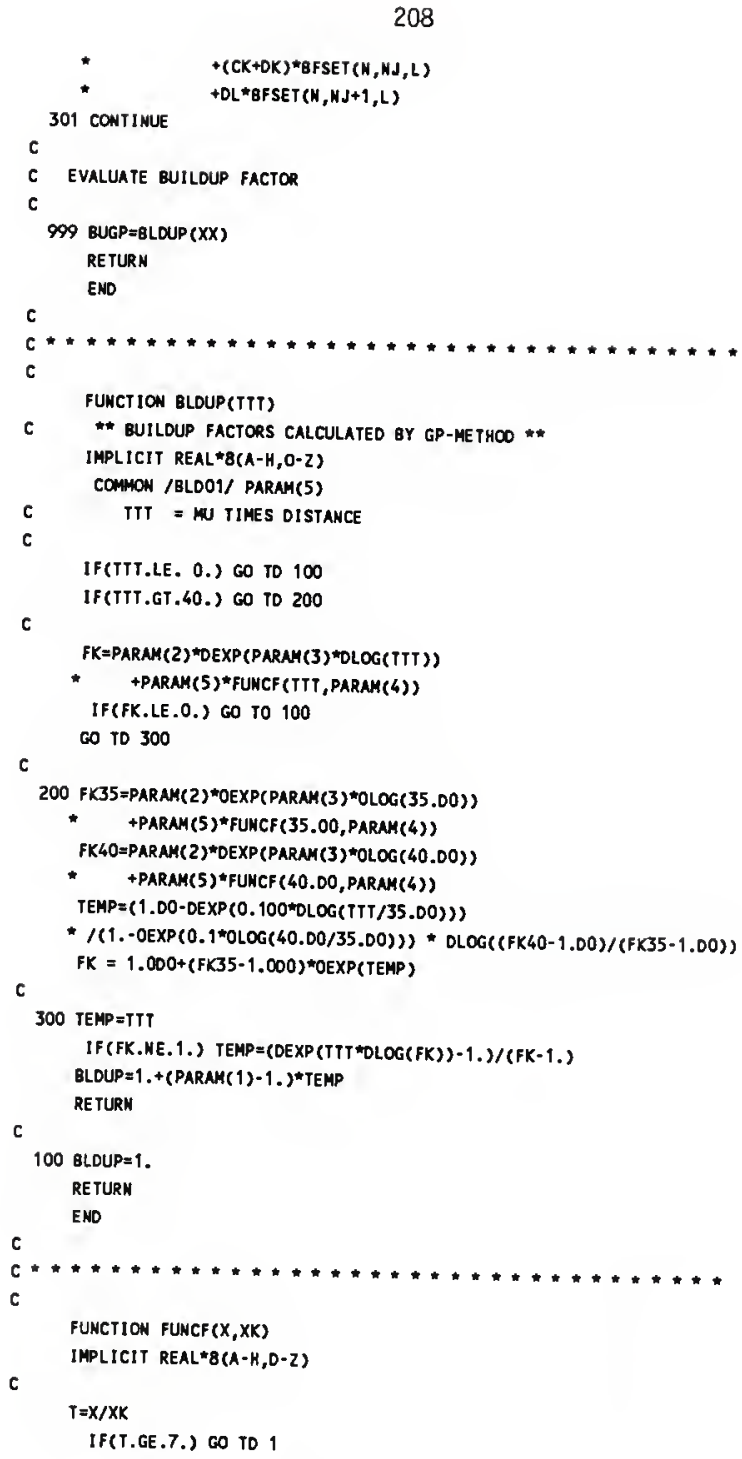


FUNCF $=1 .-(1 .+D \operatorname{EXP}(4 . D 0)) /(D E \operatorname{EXP}(2,-T)+D \operatorname{EXP}(4 . D O))$

RETURN

1 FUNCF $=1$.

RETURN

END

c

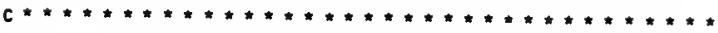




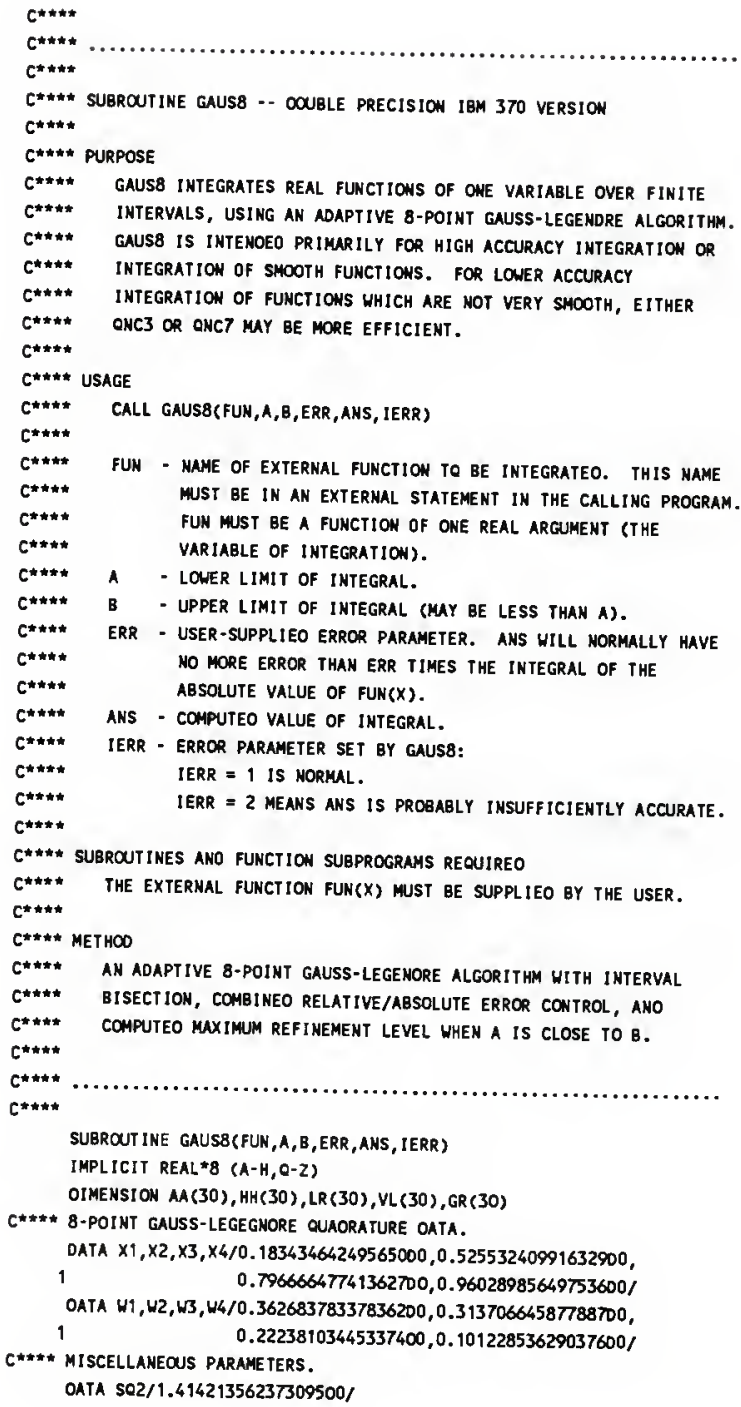


DATA LMH, NLMX, KMX, KML, NBITS/1,30,5000,6,64/

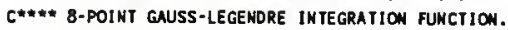

$G B(X, H)=H^{*}\left(C H C^{*}(F U N(X-X 1 * H)+F U N(X+X 1 * H))+W 2 *(F U N(X-X 2 * H)+F U N(X+X 2 *\right.$

1H) $)+\left(W^{*}\left(\right.\right.$ FUN $\left.\left.\left.\left(X-X 3^{*} H\right)+F U N\left(X+X 3^{*} H\right)\right)+W H^{*}\left(F U N\left(X-X 4^{*} H\right)+F U N\left(X+X 4^{*} H\right)\right)\right)\right)$

C*\#** INITIALIZE.

ANS $=0.0 D$

$1 E R R=1$

IF (A.EQ.B) RETURH

LMX $=$ NLMX

IF(B.EQ.0.DO) 60 TD 3

IF(DSIGN(1.DO,B)"A.LE.0.DO) CO TD 3

C=DABS $(1.00-A / B)$

IF(C.GT.0.1DO) CO TD 3

$N I B=-1 D I N T(D L O G(C) / D L O G(2 . D 0))$

LMX $=M I N O$ (NLMX, NBI TS-NIB-6)

LMX $=K H \times X$ (LMX, LMN)

$3 \mathrm{TOL}=\mathrm{DMAX} 1$ (ERR , 2.DO"* (5-NBITS))/2.DO

IF (ERR.LT.O.DO) TDL $=0.50-6$

EPS $=$ TOL

$H H(1)=(B-A) / 4.00$

$A A(1)=A$

$\operatorname{LR}(1)=1$

$L=1$

$E S T=G B(A M(L)+2.00 * H H(L), 2 . D O * H H(L))$

$K=8$

AREA $=D A B S$ (EST)

$E F=0.500$

$M X L=0$

C**** COMPUTE REFINED ESIIMATES, ESIIMATE THE ERROR, ETC.

$4 \mathrm{GL}=\mathrm{GB}(M(\mathrm{~L})+\mathrm{HH}(\mathrm{L}), \mathrm{HH}(\mathrm{L}))$

$G R(L)=G B\left(A A(L)+3.00^{*} H H(L), H H(L)\right)$

$K=K+16$

AREA $=A R E A+C O A B S(G L)+D A B S(G R(L))-D A B S(E S T))$

C IF(L.LT.LMN) $G O$ TD 11

$\mathrm{GLR}=\mathrm{GL}+\mathrm{GR}(\mathrm{L})$

EE $\triangle E F * D A B S(E S T-G L R$ )

$A E=D M A X 1$ (EPS*AREA, TDL"DABS (GLR))

IF (EE-AE) $6,6,7$

$5 \mathrm{MXL}=1$

$6 \operatorname{If}(\operatorname{LR}(L)) 8,8,10$

C**** CDNSIDER THE LEFT HALF DF THIS LEVEL.

7 IF (K.GT.KMX) LMX=KML

IF (L. GE. LMX) CO TD 5

$L=L+1$

EPS $=E P S / 2 . D 0$

$E F=E F / S O 2$

$H H(L)=H H(L-1) / 2.00$

$L R(L)=-1$

$M A(L)=M A(L-1)$

$E S T=G L$ 
co TO 4

C**** PROCEED TO RIGHT HALF AT THIS LEVEL.

$8 \mathrm{VL}(L)=\mathrm{GLR}$

9 EST $=G R(L-1)$

$L R(L)=1$

$M A(L)=M(L)+4 . D O * H H(L)$

GO TO 4

C*\#** RETURM ONE LEVEL.

10 VR=GLR

11 IF(L.LE.1) CO TO 14

$L=L-1$

EPS=EPS*2.DO

$E F=E F * S O 2$

IF(LR(L).GT.O) G0 TO 13

$V L(L)=V L(L+1)+V R$

co To 9

$13 V R=V L(L+1)+V R$

co To 11

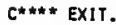

14 AHS=VR

I $F(M X L . G T$, O) IERR $=2$

RETURH

END

C****

C****

C*t* THE FOLLONING SUBROUTINES ARE DUPLICATES OF GAUS8, USED TO AVOID

C**\#* CALLIHG THE SUBROUTIME RECURSIVELY.

$c * * * *$

SUBROUTIME GAUSO(FUK, A,B,ERR, AKS, IERR)

IMPLICIT REAL*B $(A-\mathrm{H}, \mathrm{O}-\mathrm{Z})$

OIMENSIOW MA(30), HH(30), LR(30), VL (30), GR(30)

C**** B-POINT GAUSS-LEGEgNDRE OUADRATURE DATA. DATA X1,X2,X3,X4/0.18343464249565000,0.52553240991632900,

$1 \quad 0.79666647741362700,0.96028985649753600 /$

OATA W1,W2,W3,W4/0.362683783378362D0,0.31370664587788700,

$1 \quad 0.22238103445337400,0.101228536290376 \mathrm{DO} /$

C*t** MISCELLANEOUS PARAMETERS.

DATA SO2/1.41421356237309500/

DATA LMM, NLMX, KMX, KML, NBITS/1, 30,5000,6,64/

C*t* 8-POINT GAUSS-LEGENDRE IMTEGRATION FUNCIION.

$G B(X, H)=H^{*}\left(\left(W 1^{*}\left(F U N\left(X-X 1^{*} H\right)+F U N(X+X 1 * H)\right)+H 2^{*}\left(F U N\left(X-X Z^{*} H\right)+F U N\left(X+X Z^{*}\right.\right.\right.\right.$

1H) $\left.))+\left(W 3^{*}\left(F U N\left(X-X 3^{*} H\right)+F U N\left(X+X 3^{\star} H\right)\right)+W 4^{*}\left(F U N\left(X-X 4^{*} H\right)+F U N\left(X+X 4^{*} H\right)\right)\right)\right)$

C**** INITIALIZE.

ANS $=0.00$

IERR $=1$

IF (A.EO.B) RETURH

LMX $=N L M X$

IF(B.EQ.0.DO) 60 TO 3

IF (DSIGN(1.DO,B)*A.LE.0.DO) Go T0 3

$C=$ DABS $(1 . D O-A / B)$ 


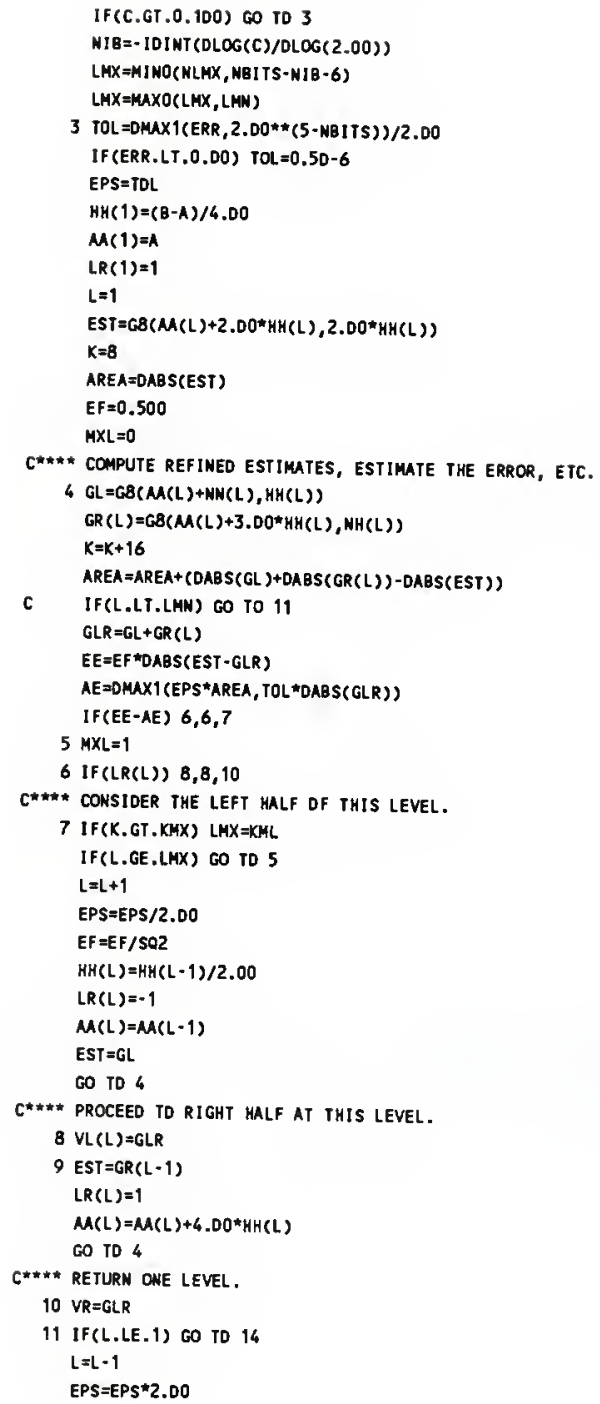




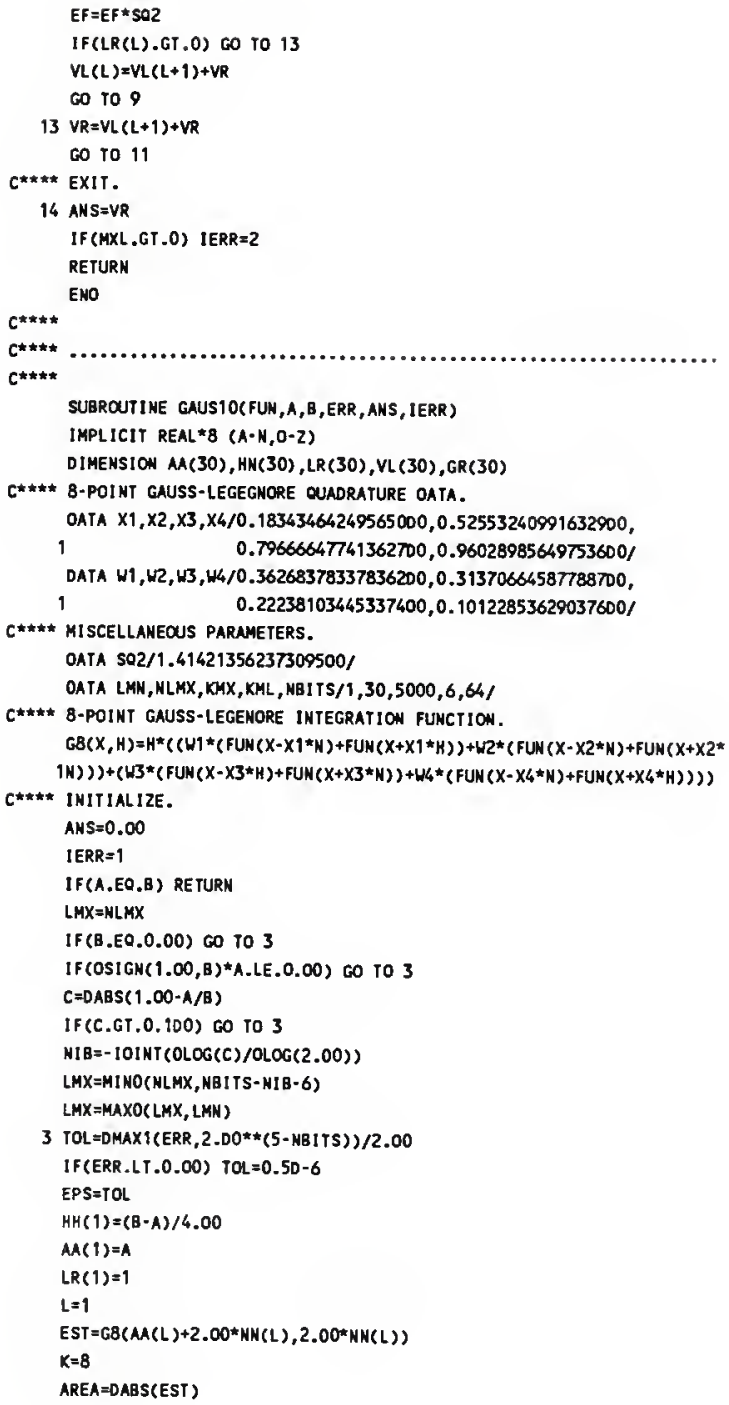




\section{5}

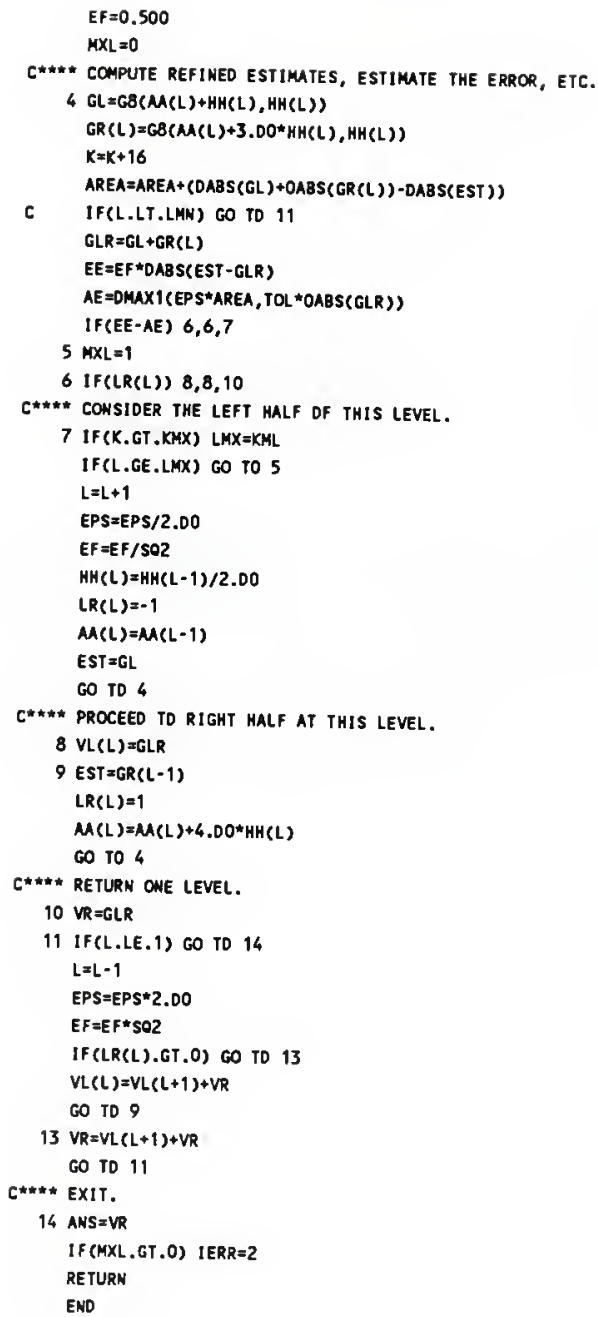




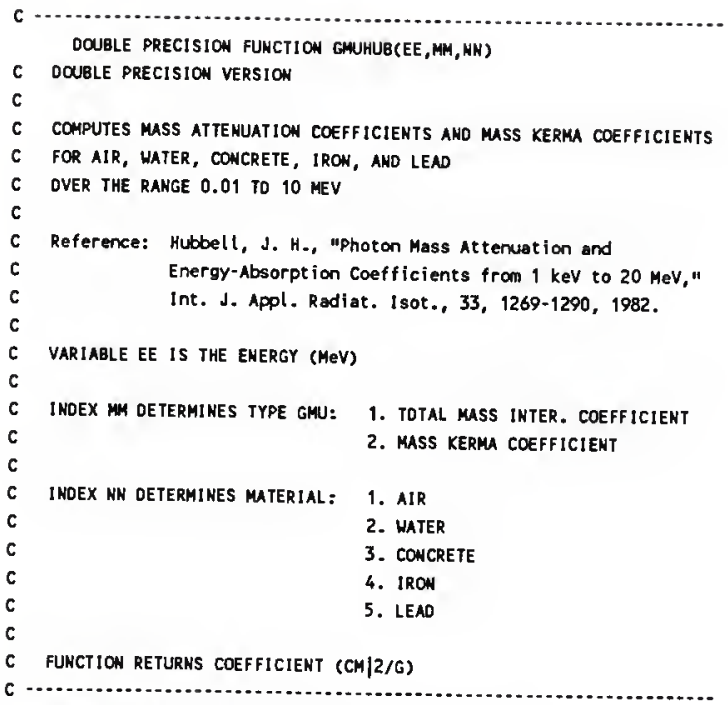

IMPLICIT REAL $* 8(A-H, O-Z)$

OIMENSION N(5), E(33,5), COEF $1(33,5), \operatorname{COEF} 2(33,5)$

C MUMBER OF ELEMENTS IN ENERGY RANGE

OATA $N / 4 * 25,33$ /

C ENERGY GROUP STRUCTURE

DATA $E / .01, .015, .02, .03, .04, .05, .06, .08, .010, .15, .2, .3, .4$

$8, .5,-6, .8,1 ., 1.5,2 ., 3 ., 4, ., 5,6 ., 8, ., 10 ., 8 * 0$.

$8, .01, .015, .02, .03, .04, .05, .06, .08, .10, .15, .2, .3, .4$

$8, .5, .6, .8,1,1,5,2,3 ., 4, .5,, 6,8,8,10 ., 8 * 0$.

$8, .01, .015, .02, .03, .04, .05, .06, .08,-10, .15, .2, .3, .4$

$8, .5, .6, .8,1 ., 1.5,2 ., 3 ., 4 ., 5 ., 6 ., 8, ., 10 ., 8 * 0$.

$8, .01, .015, .02, .03, .04, .05, .06, .08, .10, .15, .2, .3, .4$

\&..5,.6, .8, $1 ., 1.5,2 ., 3 ., 4 ., 5 ., 6 ., 8 ., 10 ., 8 * 0$.

$\$, .01, .01304, .01304, .015, .0152, .0152, .015861, .015861, .02, .03, .04$

$2, .05, .06, .08, .088004, .088004, .10, .15, .2, .3, .4, .5$

$\$, .6, .8,1 ., 1.5,2 ., 3 ., 4 ., 5 ., 6 ., 8 ., 10.1$

DATA $((\operatorname{COEF} 1(1, j), 1=1,33), J=1,1) /$

8 5.0160+00,1.5810+00,7.6430-01,3.5010-01,2.4710-01

$\&, 2.0730-01,1.8710-01,1.6610-01,1.5410-01,1.3560-01,1.2340-01$

$8,1.0680-01,9.5480-02,8.7120-02,8.0560-02,7.0750-02,6.3590-02$

$8,5.1760-02,4.4470-02,3.5810-02,3.0790-02,2.7510-02,2.5230-02$

$\&, 2.2250-02,2.0450-02,8 * 0.00 /$

DATA ( $(C O E F 1(I, J), I=1,33), J=2,2)$ /

\&.2230+00,1.6390+00,7.9580-01,3.7180-01,2.6680-01,2.2620-01

$2,2.0550-01,1.8350-01,1.7070-01,1.5040-01,1.3700-01,1.1870-01$ 
$\&, 1.0610-01,9.6870-02,8.9570-02,7.8660-02,7.0700-02,5.7550-02$ $8,4.9400-02,3.9690-02,3.4030-02,3.0310-02,2.7710-02,2.4290-02$ 8.2.2190-02,8*0.00/

DATA ( $(\operatorname{COEF} 1(I, J), I=1,33), J=3,3) /$

\& $2.6190+01,8.1850+00,3.6050+00,1,2020+00,6.0700-01,3.9180-01$ $8,2.9430-01,2.1190-01,1.7810-01,1.4330-01,1.2700-01,1.0820-01$ $8,9.6290-02,8.7670-02,8.0980-02,7,1030-02,6.3810-02,5.1970-02$ $8,4.4820-02,3.6540-02,3.1890-02,2.8950-02,2.6960-02,2.4500-02$ $8,2.3110-02,8 * 0.00 /$

DATA ( $(\operatorname{COEF} 1(I, J), I=1,33), J=4,4) /$

\& $1.6900+02,5.6560+01,2.5460+01,8.1090+00,3.6010+00,1.9440+00$ $8,1.1970+00,5.9180-01,3.7010-01,1.9600-01,1.4580-01,1.0980-01$ $8,9.3980-02,8.4130-02,7.7030-02,6.6980-02,5.9940-02,4.8830-02$ $8,4.2650-02,3.6220-02,3.3110-02,3.1460-02,3.0570-02,2.9910-02$ $8.2 .9940-02,8 * 0.00 /$ DATA $((\operatorname{COEF} 1(I, d), t=1,33), J=5,5)$ /

$\& 1.3060+02,6.1000+01,1.5800+02,1.1160+02,1.0700+02,1.4800+02$ $8,1.3200+02,1.5300+02,8.6360+01,3.0320+01,1,4360+01,8.0410+00$ $8,5.0200+00,2.4190+00,1.6100+00,7.3800+00,5.5500+00,2.0140+00$ $8,9.9850-01,4.0260-01,2.3230-01,1.6130-01,1.2480-01,8.8690-02$ $8,7.1030-02,5.2220-02,4.6070-02,4.2340-02,4.1970-02,4.2720-02$ $8,4.3910-02,4.6750-02,4.9720-02 /$

DATA $((\operatorname{COEF} 2(1, J), t=1,33), J=1,1) /$

\& $4.6400+00,1.3000+00,5.2550-01,1.5010-01,6.6940-02$

$8,4.0310-02,3.0040-02,2.3930-02,2.3180-02,2.4940-02,2.6720-02$ $8,2.8720-02,2.9490-02,2.9660-02,2.9530-02,2.8820-02,2.7870-02$ $8,2.5650-02,2.3420-02,2.0540-02,1.8660-02,1.7370-02,1.6440-02$ $8,1.5210-02,1.4460-02,8 * 0.00 /$

OATA $((\operatorname{COEF} 2(1, J), J=1,33), J=2,2) /$

$4.8400+00,1.3400+00,5.3670-01,1.5200-01,6.8030-02,4.1550-02$ $8,3.1520-02,2.5830-02,2.5390-02,2.7620-02,2.9660-02,3.1920-02$ \$, 3.2790-02,3.2990-02,3.2840-02,3.2050-02,3.1000-02,2.831D-02 \&,2.6040-02,2.2780-02,2.0630-02,1.9130-02,1.8040-02,1.6570-02 $8,1.5660-02,8 * 0.00 /$

DATA ( $(\operatorname{COEF} 2(1, \mathrm{~J}), I=1,33), \mathrm{J}=3,3)$ /

$2.4670+01,7.5820+00,3.2170+00,9.4540-01,3.9590-01,2.0480-01$ $8,1.2300-01,6.1540-02,4.1800-02,3.0140-02,2.8870-02,2.9370-02$ $8,2.9800-02,2.9840-02,2.9640-02,2.8870-02,2.7900-02,2.5540-02$ $8,2.3480-02,2.0860-02,1.9290-02,1.8280-02,1.7600-02,1.6800-02$ \&, $1.6390-02,8 * 0.00 /$ DATA ( $(\operatorname{COEF} 2(1, J), I=1,33), \mathrm{J}=4,4)$ /

\& $1.3670+02,4.8950+01,2.2570+01,7.2370+00,3.1460+00,1.6300+00$ $8,9.5380-01,4.0930-01,2.1810-01,7.9700-02,4.8400-02,3.3740-02$ $8,3.0500-02,2.9220-02,2.8430-02,2.7180-02,2.6040-02,2.3580-02$ $8,2.1950-02,2.0360-02,1.9840-02,1.9760-02,1.9910-02,2.0430-02$ 8.2.1000-02,8*0.00/ DATA $((\operatorname{COEF} 2(I, J), I=1,33), J=5,5)$ /

\& $1.2560+02,6.1000+01,1.1300+02,8.9390+01,8.0800+01,1.0900+02$ $\&, 9.8800+01,1.14 \mathrm{DE}+02,6.9230+01,2.5500+01,1.2210+01,6.7960+00$ $\$, 4.1770+00,1.9360+00,1.4500+00,2.3100+00,2.2290+00,1.1350+00$ 
2,6.2290-01,2.5810-01,1.4390-01,9.5640-02,7.1320-02,4.8380-02 2,3.7870-02,2.7140-02,2.4070-02,2.3510-02,2.4630-02,2.6000-02 2.2.7300-02,2.9480-02,3.1140-02,

C

$\mathrm{C}$

c

PERFORM INTERPOLATION

$00101 !=1, N(N N)$

If (EE.EQ.E(I, NH)) GO TO 102

IF (EE.LT.E(I, HH)) GO TO 103

101 CONTIMUE

102 IF (MH.EQ.1) CHUNUB $=\operatorname{COEF} 1(1, \mathrm{HK})$

IF(MH.EQ.2) GNUNUB $=$ COEF2(1, NN)

co To 104

$10311=1-1$

COTO $(201,202)$, АM

$201 Y Y 1=\operatorname{DLOG}(\operatorname{COEF} 1(1 I, \mathrm{NH}))$

$Y Y 2=\operatorname{OLOG}(\operatorname{COEF1}(I, H K))$

coTo 203

$202 Y Y 1=D L O C(\operatorname{COEF} 2(I I, N W))$

$Y Y 2=\operatorname{DLOG}(\operatorname{COEF} 2(1, \mathrm{HN}))$

$203 \times \times 1=\operatorname{DLOG}(E(I I, N N))$

$X X 2=\operatorname{DLOG}(E(1, K N))$

$\mathrm{GG}=\mathrm{DLOG}(\mathrm{EE})$

$22=Y Y 1+(G G-X X 1) *(Y Y 2-Y Y 1) /(X X 2-X X 1)$

GMUNUB $=\operatorname{DEXP}(Z Z)$

104 CONTINUE

RETURN

END 


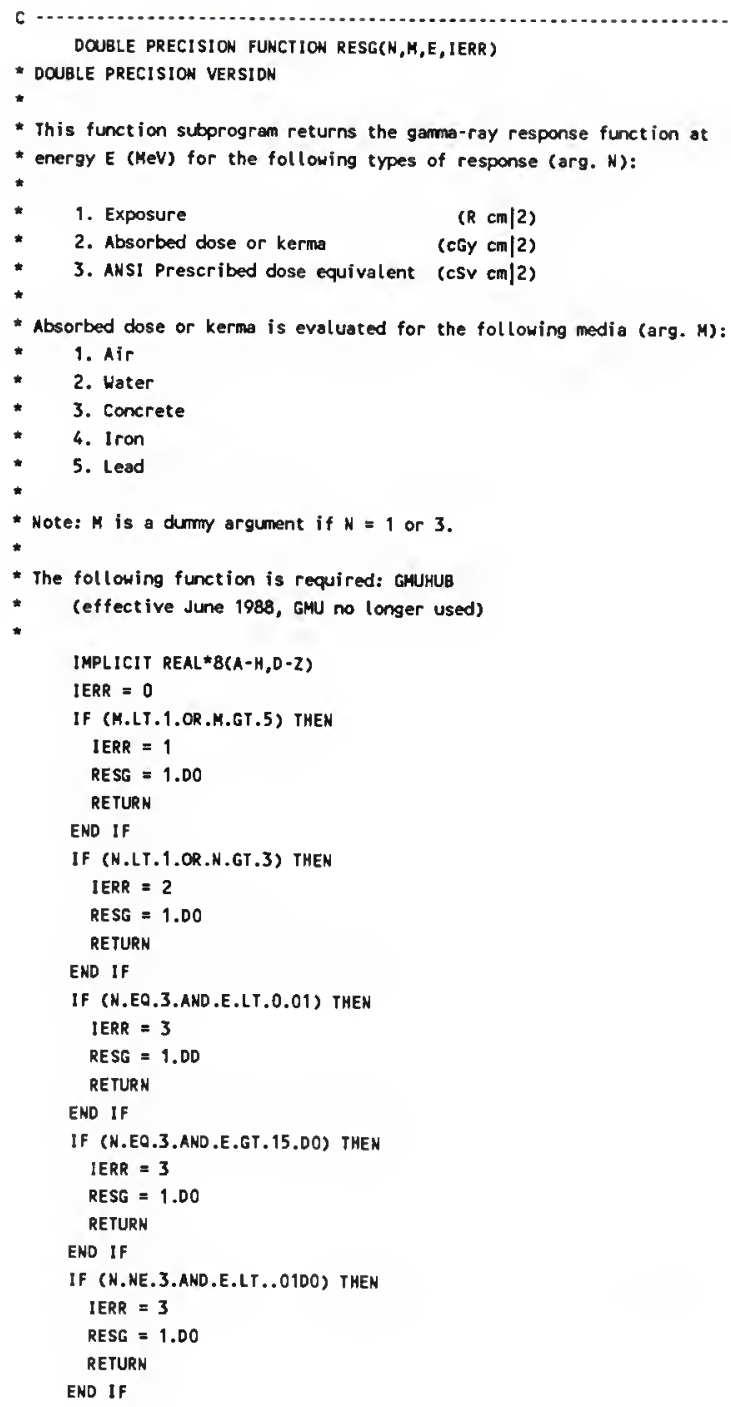


IF (N.NE.3.AND.E.GT.10.DO) THEN IERR $=3$

RESG $=1.00$

RETURN

END IF

If (N.EQ.1) THEN

RESG $=1.835 D-8^{*} E^{*} \operatorname{GMUHUB}(E, 2,1)$

RETURN

ELSE IF (N.EQ.2) TKEN

RESG $=1.6020-8{ }^{\star} E^{\star} G M U K U B(E, 2, M)$

RETURN

ELSE IF (N.EO.3) THEN

$X=\operatorname{LOG}(E)$

IF (E.LE. .03DO) THEN

RESG $=-20.47700-1.745600^{\star} X$

GO TO 10

ELSE IF(E.LE..5DD) THEM

RESG $=-13.62600-.5711700^{*} X-1.095400^{*} X * x-.2489700^{*} X^{*} X^{*} X$

GO TO 10

ELSE IF(E.LE.5.00) THEN

RESG $=-13.13300+.7200800 * x-.03360300 * x * x$

GO TO 10

ELSE

RESG $=-12.79100+.2830900 * X+.1087300^{\star} X X^{\star} X$

END IF

10

RESG=EXP (RESG )/3600.D0

RETURN

END IF

RETURN

END 


\section{APPENDIX D}

Sample Input and 0utput for the Program SIL0GP

The following is a guide for preparation of input files for the code SILOGP. In each execution of the code, a separate file is read, describing the problem geometry and other parameters. The source photon energy, silo dimensions, material properties and type of detector response specific to the problem are constant, and listed only once in the file. The detector response may be computed at any number of distances from the source in each execution. The detector positions make up the balance of the input file. 0nly one input file is accepted in each problem execution; if any quantity except the source-detector distance must be changed, a new input file and a separate execution of SILOGP will be required.

The first record of the input file lists source photon energy, angle of collimation of the silo, concrete roof thickness (if one is present), and densities of concrete and air. If the outer radius of the silo, $r$, and the height of the outer rim of the collimator above the point source, $h$, are known, the angle of collimation $\Omega$ (omega) may be

$\Omega=2 \arctan (\mathrm{r} / \mathrm{h})$.

should be specified is not used, the roof thickness and concrete density separated by commas or spaces. The remainder of the input file consists of a list of radial source-detector distances. Each distance should be placed on a separate line, and the distances need not be in any order. The values are not restricted by format; for instance, a source-detector distance of $120 \mathrm{~m}$ may be expressed as "120" or "120." or "1.2E+02."

FIRST RECORD:

$$
\begin{array}{ll}
\text { E } & \text { Source photon energy (MeV) (maximum energy } 10 \mathrm{MeV} \text { ) } \\
\text { DANG } & \text { Full angle of collimation (degrees) } \\
\text { T } & \text { Concrete thickness }(\mathrm{m}) \\
\text { RC } & \text { Concrete density }\left(\mathrm{g} / \mathrm{cm}^{3}\right) \\
\text { RAA } & \text { Air density }\left(\mathrm{mg} / \mathrm{cm}^{3}\right) \\
\text { NRESP } & \text { Type of response function: } \\
& 1=\text { exposure } \\
& 2=\text { air kerma } \\
& 3=\text { water kerma } \\
& 4=\text { AVSI prescribed dose equivalent }
\end{array}
$$

SECOND AND SUBSEQUUENT RECORDS:

D Source-detector distance $(m)$ at which response is

The sample problem, shown in Figure D-1, resembles the benchmark experiments performed at kansas State University. In the example, a cobalt-60 source is placed on the axis of a silo covered by $21 \mathrm{~cm}$ of 
concrete. In reality, ${ }^{60} \mathrm{Co}$ enits two photons per decay, of energies $1.17 \mathrm{MeV}$ and $1.33 \mathrm{MeV}$. These are approximated by two $1.25 \mathrm{MeV}$ photons for this problem. Since no collimating wedges can be placed on the silo walls when a concrete roof is present, the full angle of collimation formed by the silo is defined by interior dimensions. The point source is $31 \mathrm{~cm}$ below the roof of the silo, and the interior radius of the silo is $1.18 \mathrm{~m}$; these produce a full angle of collimation of $150.5^{\circ}$. The air density is $1.12 \mathrm{mg} / \mathrm{cm}^{3}$, while the concrete has a density of $2.13 \mathrm{~g} / \mathrm{cm}^{3}$. Exposure rates are to be computed between $30 \mathrm{~m}$ and $700 \mathrm{~m}$ from the source.

The input file for this example is shown below, and resides in the file SAMPLE S.IN on this distribution disk.

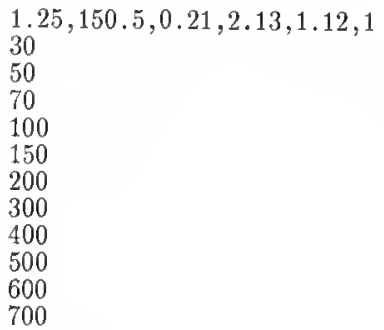

Results are given by SIL0GP as the dose rate per photon, multiplied by the square of the source-detector distance (m) and divided by the solid angle of collimation (steradians) defined by the silo. In these units, effects of inverse-square attenuation and collimation are eliminated, and results may be graphed in less space. To convert to units of exposure per unit time, multiply this result by the solid angle of collimation reported in the output, divide by the square of the source-detector distance, and multiply by the number of photons emitted per unit time. Areal density is the product of the source-to-detector distance and the air density; presenting exposure as a function of areal density compensates for variations in atmospheric temperature and pressure.

The output created by the example input file is shown below. It resides alone in the file SAMPLE-S.OUT on the distribution disk.

CALCULATION OF SKYSIINE GAMA-RAY NORHALIZED RESPONSE

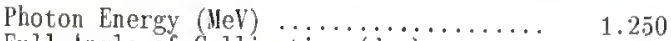

Full Angle of Collimation (deg) $\ldots \ldots \ldots 150.500$

Solid Angle of Collimation (sr) $\ldots \ldots \ldots .4 .683$

Air Density (mg/cu.cm) . ............. 1.120

Concrete Thickness (m) ............ 0.210

Concrete Density $(\mathrm{g} / \mathrm{cu} . \mathrm{cm}) \ldots \ldots \ldots \ldots \ldots, \quad 2.130$ 


\begin{tabular}{|c|c|c|c|}
\hline $\begin{array}{l}\text { SOURCE- DETECTOR } \\
\text { DISTANCE (II) }\end{array}$ & $\begin{array}{l}\text { AREAL DENSITY } \\
(G / \mathrm{CH} / 2)\end{array}$ & $\begin{array}{l}\text { NORHALIZED) } \\
(\mathrm{m} / 2 \mathrm{R} / \mathrm{sr} \text { ) }\end{array}$ & Exposure \\
\hline $\begin{array}{r}30.00 \\
50.00 \\
70.00 \\
100.00 \\
150.00 \\
200.00 \\
300.00 \\
400.00 \\
500.00 \\
600.00 \\
700.00\end{array}$ & $\begin{array}{r}3.360 \\
5.600 \\
7.840 \\
11.200 \\
16.800 \\
22.400 \\
33.600 \\
44.800 \\
56.000 \\
67.200 \\
78.400\end{array}$ & $\begin{array}{l}0.1798 \mathrm{E}-17 \\
0.2731 \mathrm{E}-17 \\
0.3468 \mathrm{E}-17 \\
0.4232 \mathrm{E}-17 \\
0.4769 \mathrm{E}-17 \\
0.4675 \mathrm{E}-17 \\
0.3634 \mathrm{E}-17 \\
0.2425 \mathrm{E}-17 \\
0.1487 \mathrm{E}-17 \\
0.8653 \mathrm{E}-18 \\
0.4865 \mathrm{E}-18\end{array}$ & $\begin{array}{ll}\text { ERROR CODE: } & 0 \\
\text { ERROR CODE: } & 0 \\
\text { ERROR CODE: } & 0 \\
\text { ERROR CODE: } & 0 \\
\text { ERROR CODE: } & 0 \\
\text { ERROR CODE: } & 0 \\
\text { ERROR CODE: } & 0 \\
\text { ERROR CODE: } & 0 \\
\text { ERROR CODE: } & 0 \\
\text { ERROR CODE: } & 0 \\
\text { ERROR CODE: } & 0\end{array}$ \\
\hline
\end{tabular}
The last column lists any error codes generated by the integration
routines in SILOGP. If the error code is nonzero, a numerical
integration performed by the code did integration performed by the code did not meet the confidence criteria, and the accuracy of the reported exposure should be questioned. 


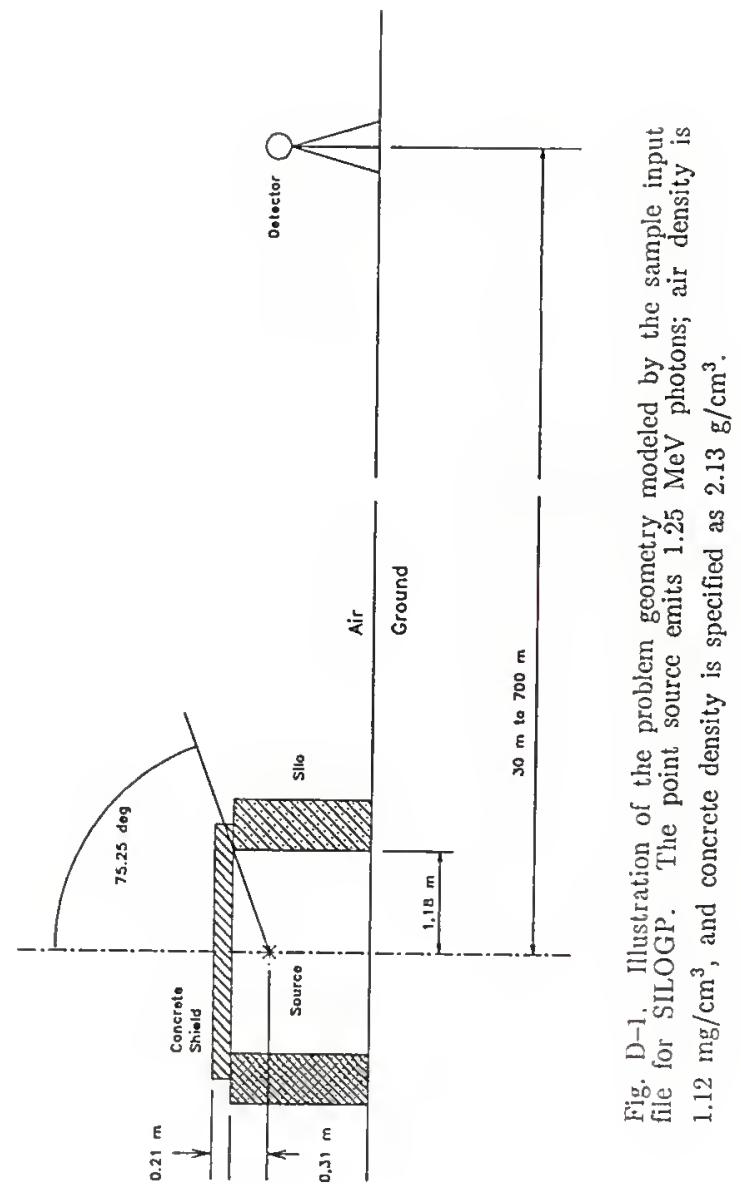




\section{APPENDIX E}

\section{Sample luput and 0utput for the Program HALLGP}

The following is a guide for preparation of input files for the code WALLGP. In each cxecution of the code, information for any number of problem geometries are read from the input file, with information for each problem on a separate line. The quantities required for each problem include source photon energy, coordinates of the source and detector with respect to the wall separating them, concrete roof thickness (if one is present), and densities of concrete and air. Also required are the type of detector response to be computed, the maximum distance in mean free paths which photons may travel, and a convergence criterion for the adaptive integration subroutines.

Some restrictions apply to the input parameters. Since the source and detector must be separated by the wall, the distances YS and YD of the source and detector below the wall must not both be negative in the same problem geometry. If no concrete roof is to be used, the roof thickness and concrete density should be specified as 0 . All quantities in each record should be separated by commas or spaces, but are not otherwise restricted by format. For instance, a source-wall distance of 120 m may be expressed as "120", "120." or "1.2E+02."

THE FOLLOWING INPUTS ARE REQUURED ON ONE LINE FOR EACH CASE:
E
XS
$X D$
IS Source distance below top of wall (m) (negati
Source energy ( $\mathrm{MeV}$ ) (maximum energy $10 \mathrm{MeV}$ )
Source-wall distance normal to the wall (m)
$Y D$ is above the wall)
Source distance below top of wall (m) (negative if source
YD Detector distance below top of wall (m) (negative if
2D Offset of detector from an axis normal to the wall
$T$ through source (m)
$T$ Thickness of concrete slab above source and wall (m)
RC Density of concrete $\left(\mathrm{g} / \mathrm{cm}^{3}\right)$
RAA Density of air ( $\mathrm{mg} / \mathrm{cm}^{3}$ )
NRESP Response function desired:
$1=\operatorname{exposure}(\mathrm{R} / \mathrm{s})$
2 = air kerma (rad/s)
3 = water kerma (rad/s)
DEL $\quad \begin{aligned} & 4=\text { dose equivalent (rem/s) } \\ & \text { dbsolute accuracy desired in integration routines }\end{aligned}$

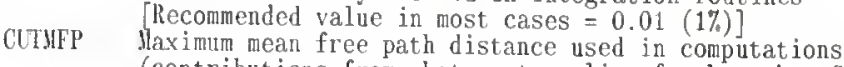
(contributions from photons traveling farther than CUTHFP are ignored; $40 \mathrm{mfp}$ absolute maximum)

In the sample problem shown in Figure E-1, a cobalt- 60 point source is placed $3 \mathrm{~m}$ behind a wall and 3 m below its top edge. The point 
detector at which exposure rates are measured is placed on the other side of the wall, $100 \mathrm{~m}$ from the wall (measured normal to the wall face) and $3 \mathrm{~m}$ below the top of the wall. A reference plane may be placed through the source and normal to the wall; the detector 1 ies $10 \mathrm{~m}$ from this plane, measured parallel to the wall. A concrete slab shield $10 \mathrm{~cm}$ thick is placed above the source, resting on the edge of the wall and extending infintely in directions behind the source and along the wall.

The two photons emitted by ${ }^{60} \mathrm{Co}$ with each decay, of energies 1.17 $\mathrm{MeV}$ and $1.33 \mathrm{MeV}$, are approximated by two $1.25 \mathrm{MeV}$ photons for this problem. The concrete has a density of $2.35 \mathrm{~g} / \mathrm{cm}^{3}$, while the air density is $1.2 \mathrm{mg} / \mathrm{cm}^{3}$. Exposure rates are to be computed at the detector; a convergence criterion of $\mathrm{DEL}=.01$ and a cutoff value of 40 mean free paths have been selected.

The record of the input file which defines this example problem is shown below. It may be found separately in the file SAMPLE- $h$. IN on this distribution disk.
1. 250
$3.0,100.0$
$3.0,3.0,10.0$
0.10
$2.35,1.2,1, .01,40$.

Results are given by WALLGP as the dose rate per second, with the source normalized to a strength of one photon emitted per second. By multiplying this result by the true source strength, the true response rate may be obtained. The output created by the input file above is shown below; this output is written by WALLG to logical unit 9 . The output is listed separately in the distribution file SAMPL-W.OUT.

\section{CALCULATION OF SKYSIINE GAMUA-RAY NORUALIZED RESPONSE}

Photon Energy (MeV) $\ldots \ldots \ldots \ldots \ldots \ldots \ldots . \ldots \ldots \ldots$

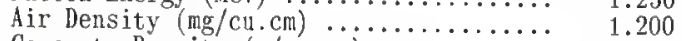

Concrete Density (g/cu.cm) ............ 2.350

Mean Free Path Cutoff Criterion ....... 40.000

Percent Error of Result ............. 1.000

$\begin{array}{lccccc}X(S) & X(D) & Y(S) & Y(D) & Z(D) & T \\ (m) & (\mathrm{m}) & (\mathrm{m}) & (\mathrm{m}) & (\mathrm{m}) & (\mathrm{m}) \\ 3.00 & 100.00 & 3.00 & 3.00 & 10.00 & 0.10\end{array}$

Normalized Exposure

Response rate * $\mathrm{d}^{* * 2} 2$

$3.8266 \mathrm{E}-21$ (R/S)

$$
\text { 4.0979E- } 17 \mathrm{~m}^{* * 2 *} \text { (R/S) }
$$

A second output file is created by KALLGP on logical unit 10 . This file contains data useful for plotting results on a graph. For each problem, one line of output is created, containing in order: detector;

1) the straight-line distance in meters between the source and 
2) the areal density (the product of distance and air density, in $\mathrm{g} / \mathrm{cm}^{2}$ ) separating the source and detector on a straight line;

3) the response rate, normalized to a source strength of one photon per second from the source;

4) the response rate, multiplied by the square of the sourcedetector distance. This value is sometimes useful in comparing results of different problem geometries, since effects of inverse-square attenuation are eliminated from the results.

The line of output in the plot data file created for this example case is shown below, and in the file SAMPLE-W.PLT.

$103.48 \quad 12.42 \quad 3.8266 \mathrm{E}-21 \quad 4.0979 \mathrm{E}-17$ 

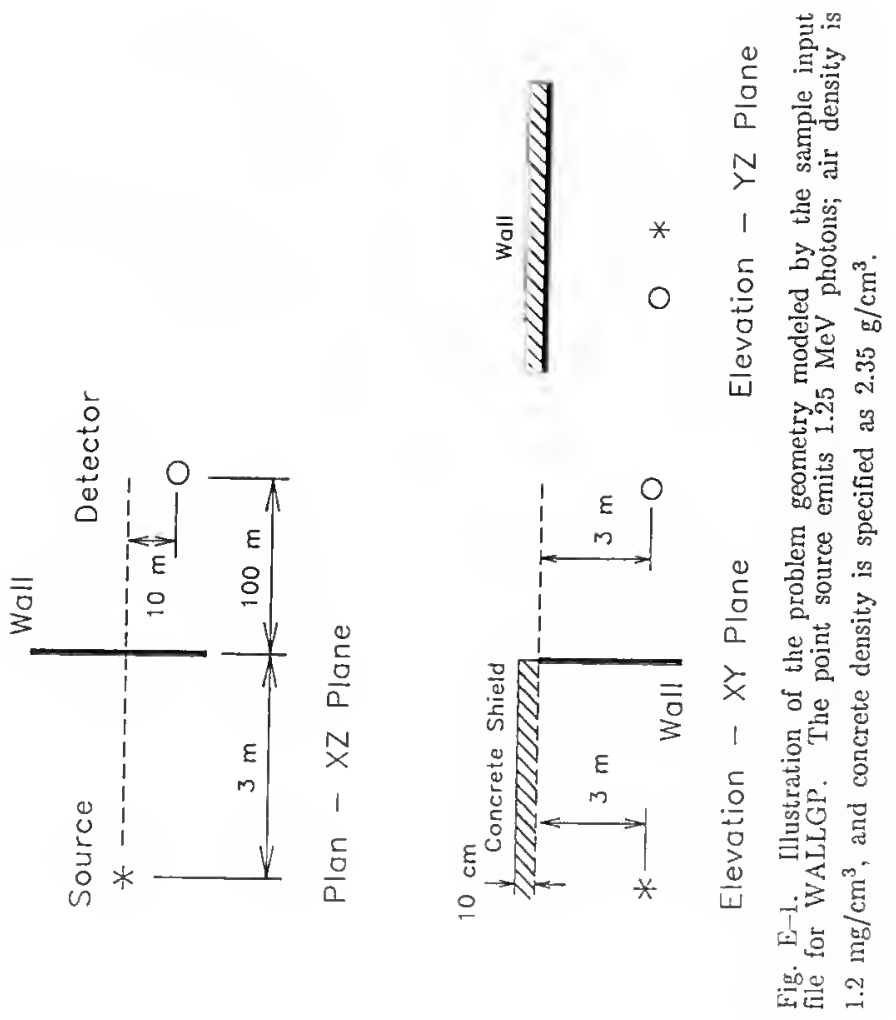
COMPUTATIONAL TECHNIQUES IN

GAMMA-RAY SIIYSHINE ANALYSIS

\author{
by \\ Darin Lynn George
}

B.S., Kansas State University, 1986

AN ABSTRACT OF A MASTER'S THESIS

submitted in partial fulfiliment of the

requirements for the degree

MASTER OF SCIENCE

Department of Nuclear Engineering

KANSAS STATE UNIVERSITY

Manhattan, Kansas

1988 


\section{ABSTRACT}

Two computer codes were developed to analyze gamma-ray skyshine, the scattering of gamma photons by air molecules. A review of previous gamma-ray skyshine studies discusses several Monte Carlo codes, programs using a single-scatter model, and the MicroSkyshine program for microcomputers. A benchmark gamma-ray skyshine experiment performed at Kansas State University is also described.

A single-scatter numericaI model was presented which traces photons from the source to their first scatter, then applies a buildup factor along a direct path from the scattering point to a detector. The FORTRAN code SKY, developed with this model before the present study, was modified to use Gauss quadrature, recent photon attenuation data and a more accurate buildup approximation. The resulting code, SILOGP, computes response from a point photon source on the axis of a silo, with and without concrete shielding over the opening. Another program, WALLGP, was developed using the same model to compute response from a point gamma source behind a perfectly absorbing wall, with and without shielding overhead.

Results of SILOGP were compared to measurements from the KSU benchmark experiment. SILOGP underpredicted the experimental exposure rates within $250 \mathrm{~m}$ of the source and overpredicted responses further away. Average deviations of SILOGP from experiment ranged from $20 \%$ to $40 \%$. Both SILOGP and WALLGP were compared to an ANSI Standard problem involving a point source in open air, and both were found to be in excellent agreement with reference values. The two codes also agreed very well with resuIts for a similar problem based on infinite-medium point-source buildup factors computed using the method of 
moments.

SILOGP and the microcomputer code MicroSkyshine were applied to several silo skyshine problems. SILOGP returned lower exposure estimates, within $30 \%$ of MicroSkyshine values in the worst cases, but within $15 \%$ in others. Microskyshine was also used to test WALLGP in comparisons which varied individual problem parameters. WALLGP consistently predicted a response at least $20 \%$ lower than that predicted by MicroSkyshine. Discrepancies between results of WALLGP and MicroSkyshine were attributed in large measure to the deliberately conservative approximations upon which the MicroSkyshine method was based. 